\title{
Regional catalogue of the spiders (Arachnida, Araneae) of Aosta Valley (NW Italy)
}

\author{
Mauro PASCHETTA \\ Laboratory di Ecologia - Ecosistemi terrestri, \\ Dipartimento di Scienze della Vita e Biologia dei Sistemi, \\ Università di Torino, Via Accademia Albertina, 13, I-10123 Torino (Italy) \\ Claretta CHRISTILLE \\ Fabio MARGUERETTAZ \\ Museo Regionale di Scienze Naturali della Valle d'Aosta, \\ Centro di Ricerca scientifico-naturalistico del Marais, \\ Fraz. Chez Borgne, l-11015 La Salle (Aosta) (Italy) \\ Marco ISAIA \\ Laboratory di Ecologia - Ecosistemi terrestri, \\ Dipartimento di Scienze della Vita e Biologia dei Sistemi, \\ Università di Torino, Via Accademia Albertina, 13, I-10123 Torino (Italy) \\ marco.isaia@unito.it (corresponding author)
}

Published on 25 March 2016

urn:Isid:zoobank.org:pub:0F3B35C3-FB21-40C4-915E-2C7C4712CD9F

Paschetta M., Christille C, Marguerettaz F. \& Isaia M. 2016. - Regional catalogue of the spiders (Arachnida, Araneae) of Aosta Valley (NW Italy). Zoosystema 38 (1): 49-125. http://dx.doi.org/10.5252/z2016n1a3

\section{ABSTRACT}

We provide the regional catalogue of the spider species of Aosta Valley (NW Italy, Graian and Pennine Alps) based on literature records and original material collected from 2011 to 2013 in the frame of a collaboration project between the University of Torino and the Regional Museum of Natural Sciences of Aosta Valley. The revision of 1162 literature records scattered in 37 papers published between 1878 and 2015 provided a list of 382 species potentially occurring in this region. On the base of taxonomical and faunistic arguments discussed in the text, we here revise the literature and exclude 74 species from the list, of which seven of unlikely occurrence and 67 in need of verification. The original data here presented raise the total number of species occurring in this region to 384, grouped in 182 genera and 36 families. For each species reported in the catalogue, we provide new collection data, previous literature records, the known distribution and the habitat in which they occurred. Compared with other northern Italian regions and considering its extent, Aosta Valley presents a moderately high number of species, hosting $26 \%$ of the species recorded in Italy. In terms of species richness, linyphiids dominate with 99 species (26\%), followed by gnaphosids (50 species,

KEY WORDS Checklist,

Alpine fauna, faunistics. $13 \%$ ), lycosids ( 45 species, $11 \%$ ) and salticids (30 species, $7 \%$ ). Wetlands host cumulatively the highest number of species, followed by shrublands, broadleaved forests and riparian habitats. Regarding chorotypes, most of the species have Palearctic distribution, followed by European and Holarctic species. Alpine endemic species represents $5 \%$ of the total. 


\author{
MOTS CLÉS \\ Catalogue, \\ faune alpine, \\ faunistiques.
}

\begin{abstract}
RÉSUMÉ
Catalogue régional des araignées (Arachnida, Araneae) de la vallée d'Aoste (nord-ouest de l'Italie).

Un catalogue régional des espèces d'araignée de la vallée d'Aoste (nord-ouest de l'Italie, Alpes grées et Alpes valaisannes) est proposé à partir des données de la littérature et du matériel nouveau collecté entre 2011 et 2013 dans le cadre d'un projet collaboratif entre l'université de Turin et le Muséum régional de Sciences naturelles d'Aoste. La révision de 1162 signalisations réparties dans 37 publications parues entre 1878 et 2015 a fourni une liste de 382 espèces potentiellement présentes dans cette région. Sur la base d'arguments faunistiques et taxonomiques discutés dans l'article, nous reprennons ici la littérature et éliminons 74 espèces de la liste: sept dont la présence est improbable et 67 qui doivent être vérifiées. Les données originales présentées ici portent le nombre total d'espèces présentes dans la région à 384, regroupées en 182 genres et 36 familles. Pour chacune des espèces citées dans le catalogue, de nouvelles données de collecte sont fournies, ainsi que les signalisations tirées de la littérature, la distribution connue et l'habitat dans lequel on la trouve. Comparées à d'autres régions de l'Italie du Nord, et compte tenu de sa superficie, la vallée d'Aoste présente un nombre d'espèes modérément élevé, abritant $26 \%$ des espèces connues pour l'Italie. En termes de richesse spécifique, les Linyphiidae dominent avec 99 espèces (26\%), suivies par les Gnaphosidae (50 espèces, $13 \%$ ), les Lycosidae ( 45 espèces, $11 \%$ ) et les Salticidae (30 espèces, $7 \%$ ). Les zones humides abritent le plus grand nombre d'espèces, suivi par les maquis, les forêts de feuillus et les habitats riverains. Concernant les chorotypes, la plupart des espèces ont une distribution paléarctique, suivies par les espèces européennes et holarctiques. Les espèces endémiques des Alpes représentent $5 \%$ de la totalité.
\end{abstract}

\section{INTRODUCTION}

Despite the small surface and the apparent landscape homogeneity, the Italian region of Aosta Valley host a relatively rich biodiversity (Bovio 2014). At least from the botanical point of view, the main factors explaining the biological diversity of this region have to be referred to the complex lithology and geological structure of the territory and to its glacial history. Moreover, the wide altitudinal range (from 300 to $4810 \mathrm{~m}$ ) allows the establishment of a complete ecological zonation and the presence of high mountains that function as barriers to perturbations from the Atlantic as well as from the Po Valley, allows the establishment of local xerothermophilic conditions, especially in the central part of the region.

More than 30\% of the regional territory is under environmental protection, with ten Natural Reserves, one National and one Regional Parks, twenty-seven Special Areas of Conservation, one Site of Community Importance, five Special Protection Areas belonging to the European ecological network Natura 2000 and four Alpine botanical gardens (Fig. 1).

In view of the recent publication of several araneological works reporting new data from this region, and in view of the recent publication of the Italian spider checklist (Pantini \& Isaia 2015), the present work aims at providing a thorough update of the spider fauna of Aosta Valley. In addition to a detailed review of the literature data, we also present new data collected in 2011-2013 in the frame of a joint research project sponsored by the Regional Museum of Natural Sciences of the Aosta Valley, University of Torino and Fondazione Goria. The project specifically aimed at deepening the knowledge on the spider fauna inhabiting wetlands and xerothermic areas, which were previously poorly investigated.

\section{MATERIAL AND METHODS}

\section{STUDY AREA}

Aosta Valley is the smallest administrative region in Italy. It is located in the upper NW corner of the country, and it encompasses almost entirely the Alpine districts of the Graian and the Pennine Alps (Marazzi 2005). It covers a surface of $3264 \mathrm{~km}^{2}$ of the mountainous land surrounding the catchment basin of Dora Baltea river. Elevation ranges from $300 \mathrm{~m}$, at the bottom of the Valley in the South-eastern part, to $4810 \mathrm{~m}$ of Monte Bianco (Mont Blanc), the highest peak of the Alps.

Due to the WE orientation of the Dora Baltea river, the region presents a particular orography, divided in two main sectors with opposite aspects. The valleys located on the left bank of the Dora Baltea (from Ferret to Gressoney Valley), presents South or South-western aspects, with high levels of solar radiation. On the contrary, the valleys located on the right bank of the Dora Baltea (from Veny to Champorcher Valley) presents North or North-eastern aspects and lower levels of solar radiation.

Although presenting a semi-continental climate, Aosta Valley is characterized by the scarcity of precipitations and by their irregular distribution: peripheral areas have high annual average, while the central part of the valley reaches very low average values (500-600 $\mathrm{mm}$ per year), thus representing a xerothermic island in the Alps.

The average altitude of about $2100 \mathrm{~m}$ a.s.l. underlines the mountainous nature of the land: only $20 \%$ of the regional area falls below $1500 \mathrm{~m}$ and nearly $60 \%$ is comprised between 1500 and $2700 \mathrm{~m}$ (the limit of the Alpine zone). The remaining $19 \%$ is represented by high alpine lands over $2700 \mathrm{~m}$ (Camerano et al. 2007). 


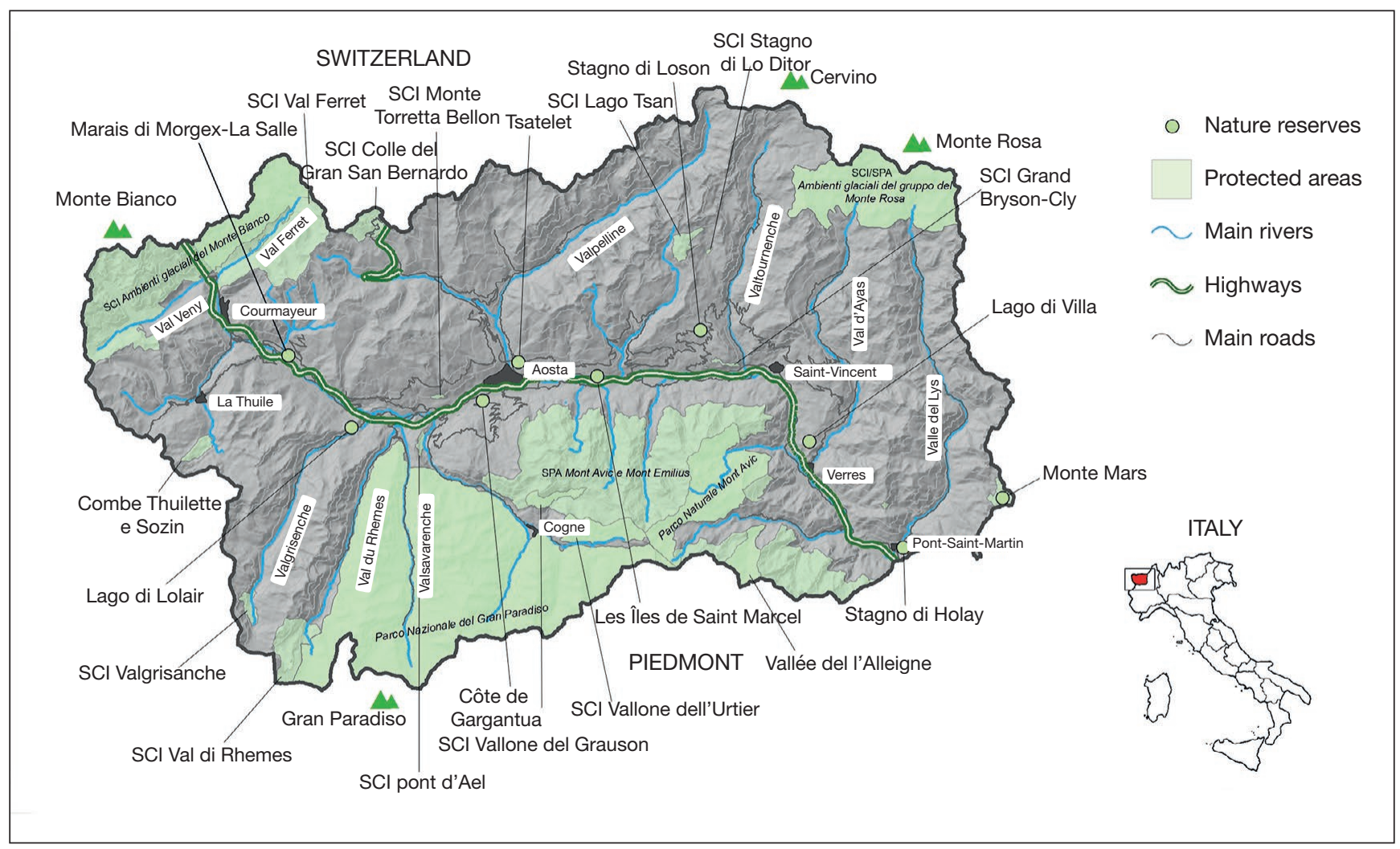

FIG. 1. - Map of Aosta Valley, with indication of Nature reserves and Protected areas.

Glaciers and rocky lands reach about $40 \%$ of the total area, representing the dominant macrohabitat, followed by forests (27\%, mainly coniferous), rupicolous grasslands $(17.4 \%$ including pseudo-steppic grasslands) and pastures (12\%) (Camerano et al. 2007). Wetlands are mainly found in the central part of the region and represent the remains of the original hygrophilous habitats once widespread throughout the whole flood plain of the main valley.

\section{SOURCES}

Literature provided a list of 382 species, scattered in 37 papers published between 1878 and 2015 (Table 1; Fig. 2). The first author reporting about spiders collected in Aosta Valley is Simon $(1878,1884)$. A few years later, Calloni (1889) published a comprehensive work on different groups of animals inhabiting the nival zone of all major Alpine districts. The author reported the presence of 57 species in different areas of Aosta Valley, named after the main peaks herein: "Gran Paradiso-Sassière-Ruitor”, "Monte Bianco", "Gran San Bernardo-Combin" and "Cervino, Rosa". Pavesi (1904) reported about 50 species collected in different localities (Gressoney St. Jean, Gressoney la Trinité, Grand St. Bernard, Petit St. Bernard, St. Rhémy, La Salle, Étroubles, La Thuile, Col du San Théodule). The work by Pavesi (1904) also includes records from bordering areas between Italy and Switzerland, which were also cited in his previous work, focusing specifically on Tessin (Switzerland) (Pavesi 1873). We consider these records valid for Aosta Valley, given the inclusion of these species in his contribution of 1904, specifically focused on the general fauna of the region. Twenty years later, di Caporiacco (1928) published a list of 54 spider species collected in Valsavaranche. After this work, the study area remained unexplored for nearly four decades.

Between 1966 and 1985 different authors published several taxonomic papers reporting material collected in Aosta Valley, providing altogether several new records for the region (Tongiorgi 1966; Lugetti \& Tongiorgi 1969; Brignoli 1971a, b; de Blauwe 1975; Brignoli 1979; Maurer 1982; Thaler 1982; Grimm 1985; Wunderlich 1985). Among these publications, it is worth mentioning the two works by Brignoli (1971b, 1979) describing Troglohyphantes nigraerosae Brignoli, 1971 (Linyphiidae), a restricted endemic subterranean species of a small sector of Graian Alps, collected at the border between Piedmont and Aosta Valley (Colle dell'Arietta). Coelotes rudolfi (Schenkel, 1925) (Agelenidae) is another Western Alpine endemic species, ranging from Lepontine to Graian Alps reported in the same period by Maurer (1982).

A decade later Rossi \& Arnò (1995) published the first faunistic study focusing on wetlands, conducted at the Natural Reserve of Les Îles de Saint Marcel. In this work, the authors reported about 50 species, most of them being new records for the region. The contribution of Fontana et al. (1996) focusing on Salticids reports the results of a survey in the Tuscan-Emilian Appennine, but also includes unpublished 
TABLE 1. - List of the publications referring to spider species collected in Aosta Valley, total number of records (Recorded species), number of new records herein reported (New records) and number of valid new records after revision (Valid new records after revision). The final number of valid records exclude uncertain species and species of doubtful occurrence (see the corresponding lists in the text).

\begin{tabular}{|c|c|c|c|}
\hline Literature & $\begin{array}{c}\text { Recorded } \\
\text { species }\end{array}$ & $\begin{array}{c}\text { New } \\
\text { records }\end{array}$ & $\begin{array}{l}\text { Valid new } \\
\text { records after } \\
\text { revision }\end{array}$ \\
\hline Simon 1878 & 3 & 3 & 1 \\
\hline Simon 1884 & 2 & 2 & 1 \\
\hline Calloni 1889 & 57 & 57 & 33 \\
\hline Pavesi 1904 & 50 & 21 & 16 \\
\hline di Caporiacco 1928 & 54 & 38 & 19 \\
\hline Tongiorgi 1966 & 3 & 1 & 1 \\
\hline Lugetti \& Tongiorgi 1969 & 5 & 3 & 3 \\
\hline Brignoli 1971a & 3 & 1 & 1 \\
\hline Brignoli 1971b & 1 & 1 & 1 \\
\hline de Blauwe 1975 & 1 & 0 & 0 \\
\hline Brignoli 1979 & 1 & 0 & 0 \\
\hline Maurer 1982 & 2 & 1 & 1 \\
\hline Thaler 1982 & 1 & 1 & 1 \\
\hline Grimm 1985 & 3 & 1 & 1 \\
\hline Wunderlich 1985 & 1 & 1 & 1 \\
\hline Rossi \& Arnò 1995 & 50 & 45 & 45 \\
\hline Fontana et al. 1996 & 9 & 6 & 6 \\
\hline Pesarini 1996 & 1 & 1 & 1 \\
\hline Pesarini 1997 & 12 & 12 & 7 \\
\hline Isaia 2000 & 30 & 13 & 13 \\
\hline Pesarini 2000 & 4 & 3 & 3 \\
\hline Arnò \& Lana 2005 & 5 & 3 & 3 \\
\hline Isaia 2005 & 261 & 52 & 52 \\
\hline De Angelis \& Fantoni 2008 & 74 & 26 & 26 \\
\hline Pantini \& Isaia 2008 & 3 & 3 & 3 \\
\hline Isaia \& Pantini 2009 & 1 & 0 & 0 \\
\hline Isaia et al. 2009 & 1 & 1 & 1 \\
\hline Negro et al. 2009 & 113 & 48 & 48 \\
\hline Gobbi et al. 2010 & 15 & 7 & 7 \\
\hline Negro et al. 2010 & 23 & 2 & 2 \\
\hline Isaia et al. 2011 & 5 & 1 & 1 \\
\hline Pesarini 2011 & 1 & 1 & 1 \\
\hline Rossi \& Bosio 2012 & 342 & 25 & 8 \\
\hline Bolzern et al. 2013 & 1 & 1 & 0 \\
\hline Negro et al. 2013 & 22 & 0 & 0 \\
\hline Rossi \& Bosio 2014 & 1 & 0 & 1 \\
\hline Isaia \& Chiarle 2015 & 1 & 0 & 0 \\
\hline Total from literature & 1162 & 382 & 308 \\
\hline Original data & 266 & 76 & 76 \\
\hline Total & & \multicolumn{2}{|c|}{384} \\
\hline
\end{tabular}

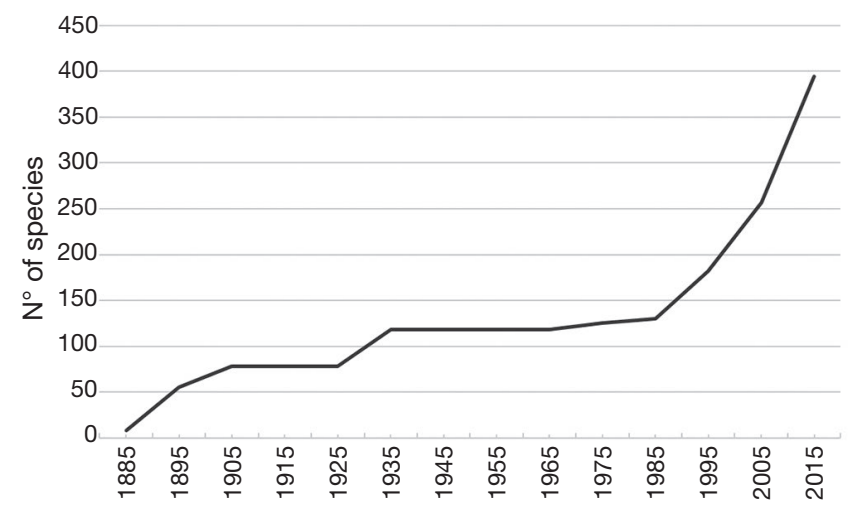

FIG. 2. - Increase in new records of spider species in the study area (1878-2015). data collected in Aosta Valley. Another contribution is given by Isaia (2000) reporting several new records collected in the Natural Park of Mont Avic between 1500 and 3000 m of altitude. The same author (Isaia 2005) published the first checklist of the region, censusing altogether 261 species including new regional records, of which one of them was new for Italy (Pardosa schenkeli Lessert, 1904). Later, De Angelis \& Fantoni (2008) focused again on the Natural Park of Mont Avic, censusing 74 species. Focusing on the ecological effects caused by ski pistes on ground dwelling arthropods in Valtournenche and Gressoney Valley, Negro et al. (2009) reported about the collection of 113 species in different habitats (pastures, ski pistes and coniferous forests). The arthropod fauna of the Miage Glacier (Veny Valley) was investigated by Gobbi et al. (2010) reporting about 15 spider species living on the glacier. Cave dwelling spiders were thoroughly investigated by Arnò \& Lana (2005) and Isaia et al. (2011) who provided a complete overview of the subterranean spiders of the region including both original data and literature records. The first update of the regional checklist was published by Rossi \& Bosio (2012), updating literature data and adding twenty-five records to the list. In 2014 the same authors published a small note about the collection of Uloborus plumipes Lucas, 1846, new for the region, in a greenhouse. This last work would bring the total number of species known for the region to 382, which decreases to 308 after the critical revision of the literature records (see Table 1 as well as the faunistical and taxonomical arguments provided in the catalogue and the paragraphs introducing the lists of uncertain and doubtful species).

\section{The Catalogue}

For each species we provide new data (New data), previous published records (Literature), distribution (Chorotype) and the habitat in which the species was actually collected in Aosta Valley by previous authors or by ourselves (Macrohabitat). Where necessary, we add taxonomical or faunistic remarks (Notes). Secondary citations (repeated records, mentioned for example in previous catalogues) are indicated in square brackets.

Concerning new data, we provide information on the collection including municipality and/or the toponym of closest locality, habitat, altitude, date (time interval in case of pitfall traps) and sampling methods. An asterisk $\left(^{*}\right)$ is added for new regional records. Unless otherwise specified, specimens were obtained using pitfall traps or by beating onto a tray and collected with a mouth aspirator. Individuals (number, sex) and collectors are also indicated.

The occurrence of 67 species (highlighted in the catalogue with the symbol ${ }^{\circ}$ ) is here questioned on the base of the arguments provided in the notes. None of these records have been validated by original data. The list mainly includes records deriving from old literature records not validated by recent data (Simon 1878, 1884; Calloni 1889; Pavesi 1904; di Caporiacco 1928), records based on alive specimen identified in the field and released after identification (Rossi \& Bosio 2012) and to several records of species not specifically reported 
for Aosta Valley but widely distributed in Italy according to Pesarini (1997) and cited in the first regional checklist of Aosta Valley (Isaia 2005).

The occurrence of seven additional species in Aosta valley (highlighted in the list with the symbol ${ }^{\circ}$ ) is here regarded as highly unlikely, uncertainty derives from taxonomic or faunistic arguments reported in the notes.

Unless otherwise indicated (MRSN-VDA: Museo Regionale di Scienze Naturali della Valle d'Aosta; MNHN: Muséum national d'Histoire naturelle de Paris), the original material is stored in the collection of Marco Isaia at the Department of Life Sciences and Systems Biology of the University of Turin.

We adopted the same chorotypes as in the Italian spider checklist (Pantini \& Isaia 2015), derived from Vigna Taglianti et al. (1993, 1999) and Stoch \& Vigna Taglianti (2005) (see Table 2).

Based on the dominant land use in the region, we identified thirteen categories of macrohabitats occurring in the study area: alpine prairies, alpine pastures, alpine grasslands, ski pistes, rocky lands, broadleaved forests, coniferous forests, riparian habitats, gravel beds, wetlands, shrublands, caves, synanthropic habitats.

"Alpine prairies" are ungrazed or lightly grazed grasslands above $1900 \mathrm{~m}$, while "alpine pastures" are grazed grasslands at intermediate elevations (1600-1900 m). On the other hand, "alpine grasslands" are grazed grasslands below $1600 \mathrm{~m}$. The category "ski pistes" includes anthropogenic environments created by the abrupt clear-cutting of alpine prairies and coniferous forests for the establishment of ski pistes. "Rocky lands" includes alpine screes over $2000 \mathrm{~m}$ a.s.l. and rocky slopes at lower elevations. "Broadleaved forests" include forests with Fagus sylvatica L., Castanea sativa Mill. and Quercus pubescens Willd. "Coniferous forests" comprises forests Abies alba Mill., Pinus mugo subsp. uncinata (Ramond ex DC.) Domin, 1936, Larix decidua Mill. and Pinus sylvestris L. We grouped riparian vegetation and riparian forests (Fraxinus angustifolia Vahl, Alnus glutinosa (L.) Gaertn., Salix alba L., Populus alba L., Alnus incana (L.) Moench) under the category "riparian habitats". "Gravel beds" includes gravel bed of rivers without vegetation. Peat bogs, reed-beds, lake-shores and mountain springs are included in the category "wetlands". Grasslands with bushes and submediterranean shrublands characterized by xerothermic conditions are assigned to the "shrublands" category. The study area encompasses several subterranean systems such as natural caves, military bunkers and abandoned mines. These habitats are included in the "caves" category. The category "synanthropic habitats" includes areas altered by persistent human activities: houses, dry stone walls, bridges, agricultural areas (such as vineyards and orchards) and gardens.

The catalogue follows the alphabetical order and nomenclature of the World Spider Catalog, Version 16 (2015).

For the sake of clarity, all species considered in the present work are presented in Appendix, highlighting new records, species of unlikely occurrence and species whose presence in the study area is plausible but need verification.
TABLE 2. - Chorotypes, number of species per chorotype and corresponding groups of chorotypes used in Fig. 18. Chorotypes follow Pantini \& Isaia (2015) derived from Vigna Taglianti et al. $(1993,1999)$ and Stoch \& Vigna Taglianti $(2005)$

\begin{tabular}{lcc}
\hline Chorotype & Abbreviation & N Group (Fig. 18) \\
\hline Cosmpolitan & COS & 6 Cosmopolitan \\
Subcosmopolitan & SCO & 1 \\
\hline Holarctic & OLA & 63 Holarctic \\
\hline Palearctic & PAL & 118 Palearctic \\
Sibirian-European & SIE & 50 \\
Central Asia-European & CAE & 4 \\
Central Asia-Mediterranean & CAM & 1 \\
Asiatic-European & ASE & 17 \\
\hline Turanic-European & TUE & 10 Turanic- \\
Turanic-Mediterranean & TUM & 2 \\
\hline European & & \\
Southern European & EUR & 55 European- \\
Central European & SEU & 22 \\
European-Mediterranean & CEU & 6 \\
Western European & EUM & 4 \\
Mediterranean & WEU & 1 \\
\hline Alpine & MED & 6 Mediterranean \\
Southern-Central Alpine & ALP & 6 Endemic \\
Western Alpine & ALPC & 2 \\
Southwestern Alps-Northern & AWNA & 8 \\
$\quad$ Appenine & & \\
\hline Total & & 384 \\
\hline
\end{tabular}

\section{ANNOTATED REGIONAL CATALOGUE OF THE SPIDERS OF AOSTA VALLEY}

Family Agelenidae C. L. Koch, 1837

Agelena labyrinthica (Clerck, 1757)

(Fig. 3)

NeW DATA. - o Donnas: Pramotton, $322 \mathrm{~m}$, in the hedge of a garden 27.VII.2009, 1 \% , leg. Paschetta.

Literature. — Pesarini 1997; [Isaia 2005].

Chorotype. - PAL.

MACROHABITAT. - Synantropic habitats.

NOTES. - Isaia (2005) listed this species in his previous regional checklist on the base of Pesarini (1997), who did not provided specifical records for Aosta Alley but reported it for the whole of Italy. On this base, Rossi \& Bosio (2012) considered it doubtful. The occurrence of the species in Aosta Valley is here validated by new original data.

\section{Allagelena gracilens (C. L. Koch, 1841)}

NEW DATA. - Morgex-La Salle: Natural Reserve of Marais, 900 m, wet meadow, 16.VIII.2011, 1 ơ, leg. Paschetta \& Giuliano.

Literature. - Rossi \& Arnò 1995; [Isaia 2005]; [Rossi \& Bosio 2012]. 
Chorotype. - SIE.

MACROHABITAT. - Riparian habitats, shrublands, wetlands.

${ }^{\circ}$ Coelotes atropos (Walckenaer, 1830)

LiTERATURE. - Calloni 1889.

Chorotype. - EUR.

MacrohabitAT. - Not specified.

Notes. - According to Brignoli (1977) and to Pantini \& Isaia (2015), this species does not occur in Italy and Calloni's record should be regarded as a misidentification. Moreover Calloni (1889) reported the record of Coelotes roscidus L. Koch, 1868 [= Pireneitega segestriformis (Dufour, 1820)] and C. solitarius L. Koch, 1868. The occurrence of both species in Aosta Valley is not plausible given their distribution, the first being distributed in the Pyrenees and the latter in the Eastern Alps (Germany, Austria and Slovenia). The occurrence of these species in Aosta Valley is also considered doubtful by Rossi \& Bosio (2012).

\section{Coelotes mediocris Kulczyński, 1887}

NEW DATA. - Torgnon: Lo Ditor, 1950 m, peat bog, 11-26. VI.2013, 1 \&, leg. Negro; same locality, 26.VII-14.VIII.2013, 1 o", leg. Negro (MRSN-VDA).

Literature. - De Angelis \& Fantoni 2008; Negro et al. 2009; [Rossi \& Bosio 2012].

Chorotype. - EUR.

MACROHABITAT. - Wetlands, broadleaved forests.

\section{- Coelotes pabulator Simon, 1875}

Literature. - Di Caporiacco 1928; [Isaia 2005]; [Rossi \& Bosio 2012].

CHorotype. - ALPW.

MACROHABITAT. - Not specified.

NOTES. - Old record in need of validation.

Coelotes pickardi O. P.-Cambridge, 1873

Literature. - Di Caporiacco 1928; de Blauwe 1975; Maurer 1982; Isaia 2000, [2005]; Isaia \& Pantini 2009; Negro et al. 2010, 2013; [Rossi \& Bosio 2012].

Chorotype. - ALPW.

MACROHABITAT. - Ski pistes, alpine prairies, rocky lands.

Notes. - See Isaia \& Pantini (2009) about the nomenclature issues related to C. pickardi and C. pastor Simon, 1875.
Coelotes rudolfi (Schenkel, 1925)

LiterATURE. - Maurer 1982; [Isaia 2005]; Negro et al. 2009, 2013; [Rossi \& Bosio 2012].

Chorotype. - ALPW.

Macrohabitat. - Alpine prairies, ski pistes.

\section{Eratigena agrestis (Walckenaer, 1802)}

NEW DATA. - Saint Denis: Brison-Cly, $1000 \mathrm{~m}$, dry grassland, 25.VII-20.VIII.2012, 1 ơ, leg. Negro; Saint-Marcel, Quart, Nus: Natural Reserve of Les Îles, 530 m, dry meadow, 18.VIII-16.IX.2011, $10^{7}$, leg. Paschetta \& Giuliano.

Literature. — Brignoli 1971a; [Isaia 2005]; [Rossi \& Bosio 2012].

Chorotype. - EUR.

MACROHABITAT. - Shrublands.

\section{Histopona leonardoi}

Bolzern, Pantini \& Isaia, 2013

NEW DATA. - Torgnon: Lo Ditor, 1950 m, peat bog, 26.VI-12. VII.2013, 1 ठ , leg. Negro; same locality, 26.VII-14.VIII.2013, $10^{7}$; same locality, 14-28.VIII.2013, $10^{7}$, leg. Negro (MRSNVDA); Ayas: Antagnod, 1970-2070 m, coniferous forests, 2007, leg. Franco \& Negro.

Literature. - De Angelis \& Fantoni 2008; Negro et al. 2009, 2010, 2013; [Rossi \& Bosio 2012]; [Bolzern et al. 2013].

Chorotype. - AWNA.

MACROHABITAT. - Wetlands, alpine prairies, broadleaved forests, coniferous forests.

Notes. - Specimen from Aosta Valley identified before 2013 were assigned to $H$. italica Brignoli, 1977 by previous authors and re-assigned to $H$. leonardoi by Bolzern et al. (2013).

•Inermocoelotes inermis (L. Koch, 1855)

Literature. - Di Caporiacco 1928; [Rossi \& Bosio 2012].

Chorotype. - SEU.

MACROHABITAT. - Rocky lands, alpine prairies.

NOTES. - Old record in need of validation.

-Tegenaria domestica (Clerck, 1757)

LiterATURE. - Rossi \& Bosio 2012.

Chorotype. - COS.

MaCrohabitaT. - Synantropic habitats.

Notes. - Uncertain record, reported by Rossi \& Bosio (2012) on the base of live specimen, released after identification. As recommended by several authors (Locket \& Millidge 1953; Roberts 1985, 


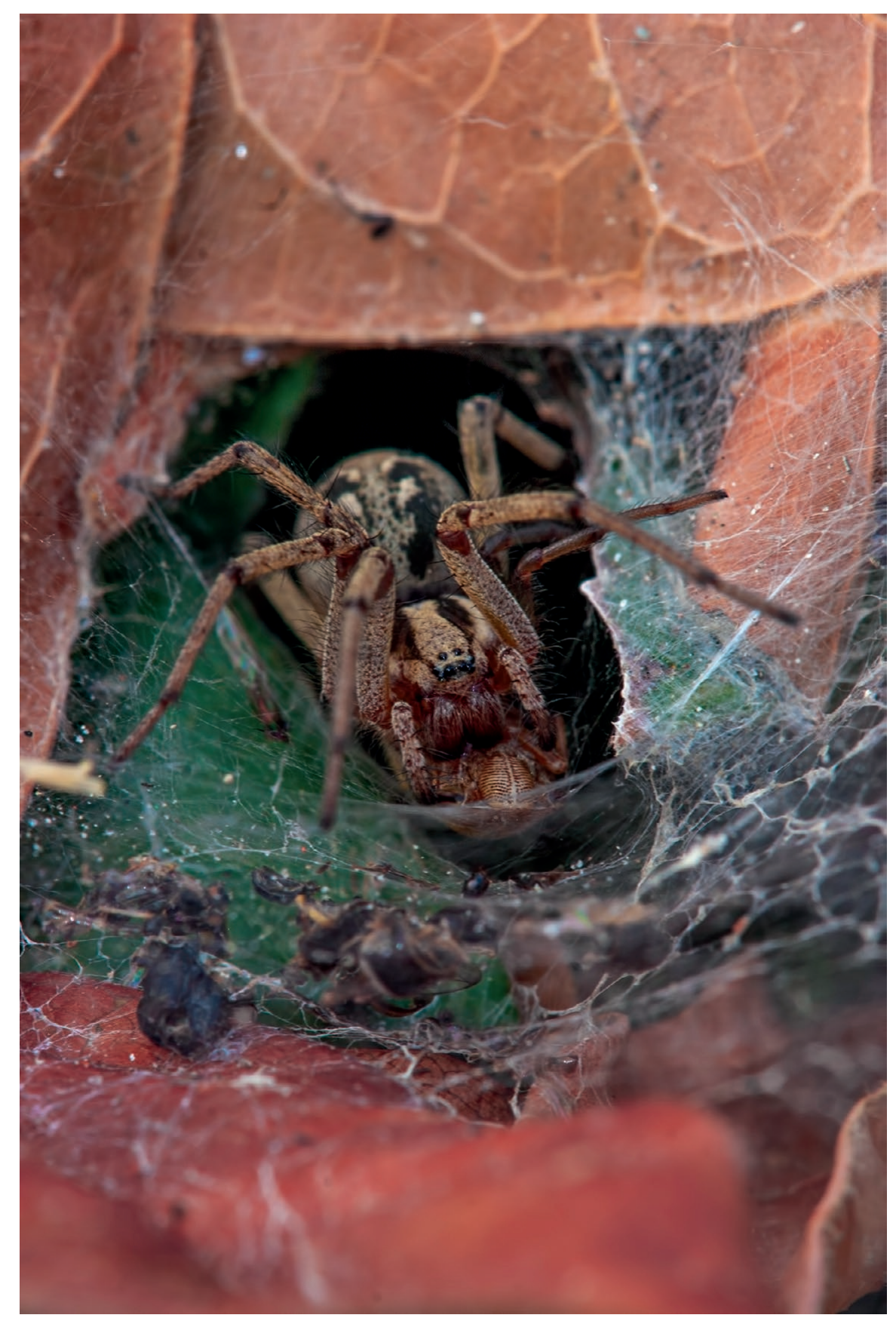

FIG. 3. - Agelena labyrinthica (Clerck, 1757) (Agelenidae), female. Average body length of the female: 10-16.5 mm. Donnas: Pramotton. Photograph: Mauro Paschetta 2009.

$1995)$ and as a general rule among arachnologists, in-field identification is generally uncertain and only a few numbers of species can be identified with a tolerable certainty. Given that no specimen is stored in any collection, the record can not be verified.

\section{Tegenaria parietina (Fourcroy, 1785)}

Literature. — Pavesi 1904; Brignoli 1971a; [Isaia 2005]; [Rossi \& Bosio 2012].

Chorotype. - SCO.

Macrohabitat. - Not specified.
Tegenaria silvestris L. Koch, 1872

New DATA. - Aymavilles: Pont d'Ael, 800 m, dry grasslands, 3-24.V.2012, 1 ơ $^{7}$, leg. Negro; Arvier: Lago di Lolair, 1175 m, broadleaved forest, 3-24.V.2012, 1 \%, leg. Negro; same locality, 24.V-13.VI.2012, 1 \% , leg. Negro; same locality, 13.VI-4.VII.2012, $10^{7}$, leg. Negro; same locality, 25.VII-20.VIII.2012, 2 o", leg. Negro; Morgex-La Salle: natural reserve of Marais, $900 \mathrm{~m}$, riparian forest, 1-26.VI.2013, 1 ㅇ, leg. Negro; same locality, 900 m, riparian forest, 18.VIII-16.IX.2011, 1 \%, leg. Paschetta \& Giuliano.

LiTERATURE. - Pavesi 1904; Brignoli 1971a; [Isaia 2005]; Negro et al. 2009; [Rossi \& Bosio 2012]. 
Chorotype. - EUR.

MACROHABITAT. — Shrublands, riparian habitats, broadleaved forests.

\section{Textrix denticulata (Olivier, 1789)}

NEW DATA. - Saint Christophe: Tsatelet, $750 \mathrm{~m}$, broadleaved forest, 3-24.V.2012, 1 O' $^{\prime \prime}$ leg. Negro; same locality, 24.V-13.VI.2012, 3 o" 1 , leg. Negro; same locality, 13.VI-4.VII.2012, 10 ơ 3 ㅇ, leg. Negro; same locality, 4-25.VII.2012, 4 o 1 ㅇ, leg. Negro; Challand-SaintVictor/Montjovet: Natural Reserve of Lago di Villa, $820 \mathrm{~m}$, broadleaved forest, 26.VII-14.VIII.2013, 1 ơ, leg. Negro; Saint Denis: Brison-Cly, 1000 m, dry grassland, 4-25.VII.2012, 1 O', leg. Negro, (MRSN-VDA); Pont-Saint-Martin: Natural Reserve of Stagno di Holay, 702 m, meadow, 26.VI-12.VII.2013, 1 o", leg. Negro; same locality, broadleaved forest (Castanea sativa), 26.VII-14.VIII.2013, 1 \% , leg. Negro; Arvier: Lago di Lolair, 1175 m, broadleaved forest, 13.VI-4.VII.2012, 1 o", leg. Negro; same locality, 4-25.VII.2012, $10^{\prime \prime}$, leg. Negro.

Literature. - Isaia 2000, [2005]; De Angelis \& Fantoni 2008; [Rossi \& Bosio 2012].

Chorotype. - EUR.

MACROHABITAT. - Broadleaved forests, shrublands, synanthropic habitats.

Family AMAUROBIIDAE Thorell, 1870

Amaurobius fenestralis (Ström, 1768)

NEW DATA. - Ayas: Antagnod, 1970-2070 m, coniferous forests, 2007, leg. Franco \& Negro.

Literature. - Pavesi 1904; [Isaia 2005]; De Angelis \& Fantoni 2008; Gobbi et al. 2010; [Rossi \& Bosio 2012].

ChOrotype. - EUR.

MacrohabitaT. - Coniferous forests, synanthropic habitats, rocky lands.

\section{Amaurobius ferox (Walckenaer, 1830)}

NEW DATA. - Gressan: Natural Reserve Côte de Gargantua, 700 m,

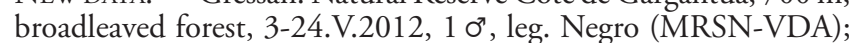
Arvier: Lago di Lolair, 1175 m, broadleaved forest, 13.VI-4.VII.2012, 1 , leg. Negro.

LiTeRATURE. - [Isaia 2005]; Arnò \& Lana 2005; Isaia et al. 2011; [Rossi \& Bosio 2012].

Chorotype. - OLA.

MACROHABITAT. - Broadleaved forests, caves.

\section{Amaurobius jugorum L. Koch, 1868}

NEW DATA. - Arvier: Lago di Lolair, $1175 \mathrm{~m}$, broadleaved forest, 3-24.V.2012, 10 o", leg. Negro (MRSN-VDA); same locality, 3-24.V.2012, 1 \%, leg. Negro (MNHN); same locality, 24.V-13.

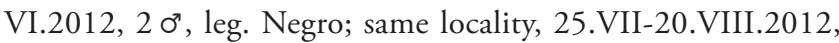
1 , leg. Negro; Gressan: Natural Reserve Natural Reserve of Côte de Gargantua, $700 \mathrm{~m}$, broadleaved forest, 3-24.V.2012, $20^{7} 1$ \%, leg. Negro; Pont-Saint-Martin: Natural Reserve of Stagno di Holay, $702 \mathrm{~m}$, broadleaved forest (Castanea sativa), 11-26.VI.2013, $2 \mathrm{O}^{7}$ 1 , leg. Negro.

Literature. - Pavesi 1904; [Isaia 2005]; [Rossi \& Bosio 2012].

Chorotype. - SEU.

MacrohabitAT. — Broadleaved forests.

*Amaurobius similis (Blackwall, 1861)

NEW DATA. - Courmayeur: Val Ferret, 1779 m, shrubs, 28.VIII16.IX.2013, 1 ơ, leg. Negro.

Chorotype. - OLA.

MACROHABITAT. - Shrublands.

Notes. - New record for Aosta Valley.

Family ANYPHAENIDAE Bertkau, 1878

Anyphaena accentuata (Walckenaer, 1802)

Literature. - Isaia 2005; [Rossi \& Bosio 2012].

Chorotype. - TUE.

MacrohabitaT. - Not specified.

Family ARANEIDAE Clerck, 1757

Aculepeira carbonaria (L. Koch, 1869)

LiterATURE. - Calloni 1889; di Caporiacco 1928; Isaia 2000; [Isaia 2005]; [Rossi \& Bosio 2012].

Chorotype. - PAL.

MACROHABITAT. - Rocky lands.

\section{Aculepeira ceropegia (Walckenaer, 1802)}

NEW DATA. — Courmayeur: Val Ferret, 1700 m, peat bog, 11.VII.2012, $3 \mathrm{o}^{\text {" }}$, leg. Paschetta, Giuliano \& Mammola; same locality, grove of reeds, 11.VII.2012, 2 o 3 \% , leg. Paschetta, Giuliano \& Mammola; same locality, peat bog, 11.VII.2012, 2 \%, leg. Paschetta, Giuliano \& Mammola; La Thuile: Comba Thuilette, $2000 \mathrm{~m}$, wet grassland, 9.VII.2012, $10^{\prime \prime} 2$ ㅇ, leg. Paschetta; same locality, rocky lands, 10.VII.2012, 1 o $^{\text {T }} 1$ \%, leg. Paschetta, Giuliano, Mammola \& Marguerettaz; Ayas: Antagnod, 1970-2070 m, alpine pasture, 2007, leg. Franco \& Negro.

Literature. - Calloni 1889; Pavesi 1904; di Caporiacco 1928; Isaia 2000; [Isaia 2005]; Gobbi et al. 2010; [Rossi \& Bosio 2012].

Chorotype. - PAL.

MACROHABITAT. - Wetlands, rocky lands, alpine prairies, alpine pastures. 
Agalenatea redii (Scopoli, 1763)

Literature. — Isaia 2005; [Rossi \& Bosio 2012].

CHORotyPe. - PAL.

Macrohabitat. - Not specified.

Araneus angulatus Clerck, 1757

(Fig. 4)

NEW DATA. - Brissogne: Saint-Marcel, Quart, Nus: Natural Reserve of Les îles, $530 \mathrm{~m}$, grove of reeds, 17.VIII.2011, 1 \%, leg. Paschetta \& Giuliano.

Literature. - Rossi \& Arnò 1995; [Isaia 2005]; [Rossi \& Bosio 2012].

Chorotype. - PAL.

MACROHABITAT. - Broadleaved forests, wetlands.

Araneus circe (Audouin, 1826)

Literature. - Isaia 2005; [Rossi \& Bosio 2012].

CHOROTYPe. - EUR.

MACROHABITAT. - Synanthropic habitats.

\section{Araneus diadematus Clerck, 1757}

NEW DATA. - Fontainemore: Monte Mars, 1900 m, rocky land, 14.IX.2012, 1 \% , leg. Paschetta \& Mammola; Challand-Saint-Victor: Natural Reserve of Lago di Villa, 820 m, grove of reeds, 16.IX.2011, 1 \&, leg. Paschetta; same locality, 14.IX.2012, 1 \%, leg. Paschetta; Pont-Saint-Martin: Natural Reserve of Stagno di Holay, 616 m, alpine grassland, 14-28.VIII.2013, 1 ơ, leg. Negro.

Literature. - Calloni 1889; di Caporiacco 1928; Isaia 2000; [Isaia 2005]; De Angelis \& Fantoni 2008; [Rossi \& Bosio 2012].

Chorotype. - OLA.

MACROHABITAT. - Rocky lands, wetlands, alpine prairies, alpine grasslands, synanthropic habitats.

\section{-Araneus marmoreus Clerck, 1757}

LiTERATURE. — Pavesi 1904; [Isaia 2005].

Chorotype. - OLA.

MACROHABITAT. - Not specified.

NOTES. - Old record in need of validation. The occurrence of this species in Aosta Valley is considered doubtful by Rossi \& Bosio (2012).

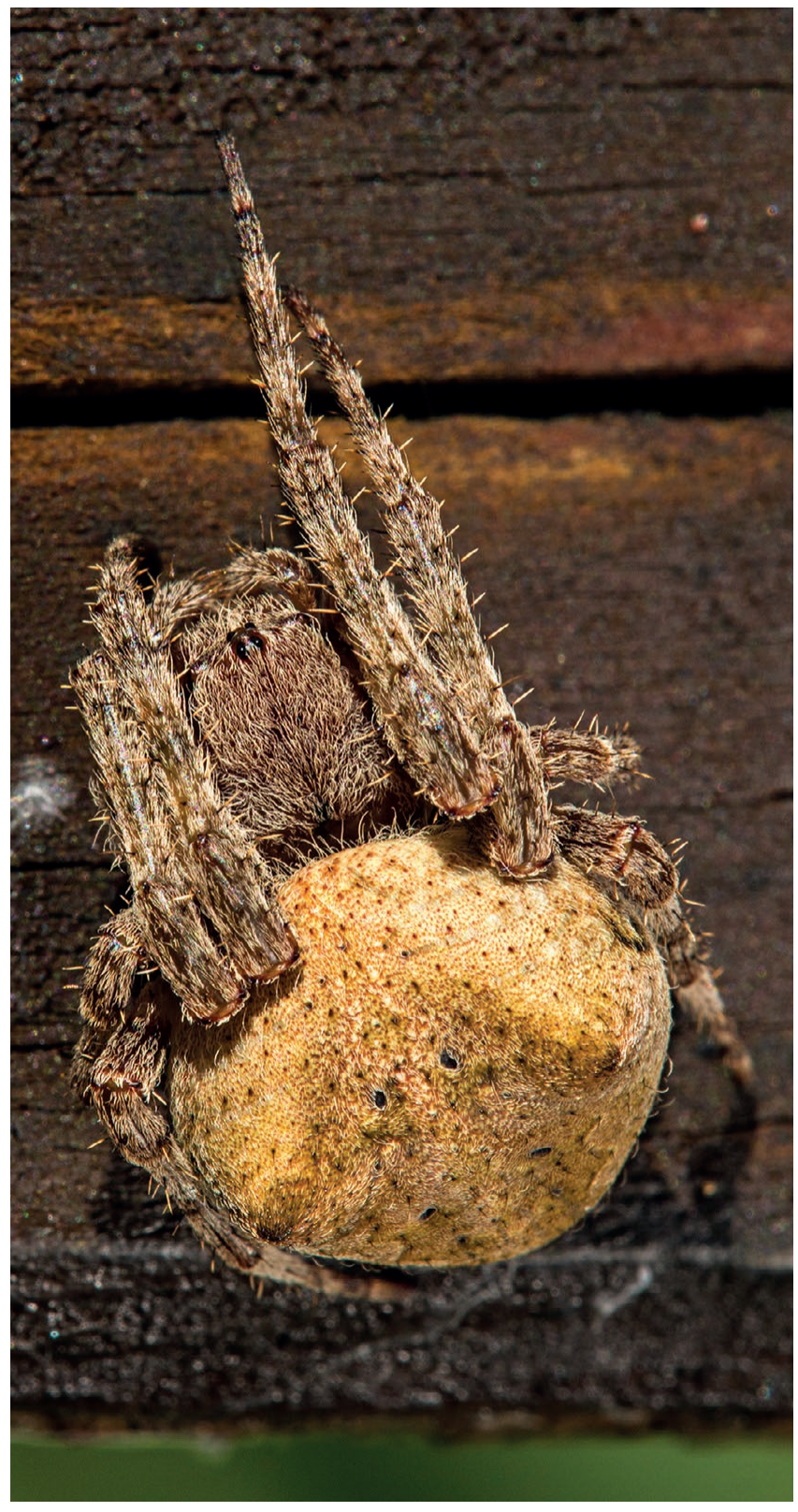

FIG. 4. - Araneus angulatus Clerck, 1757 (Araneidae), female. Average body length of the female: 15.5-20 mm. Natural Reserve of Les îles de Saint Marcel. Photograph: Mauro Paschetta 2011.

Araneus quadratus Clerck, 1757 (Fig. 5)

NEW DATA. — La Thuile: Comba Thuilette, 2000 m, rocky lands, 10.VII.2012, 1 ơ, leg. Paschetta, Giuliano, Mammola, \& Marguerettaz; same locality, 13.IX.2012, 1 ㅇ, leg. Paschetta, Mammola \& Marguerettaz; Courmayeur: Val Ferret, 1700 m, grove of reeds, 11.VII.2012, 1 ơ 1 \%, leg. Paschetta, Giuliano \& Mammola.

LiTERATURE. - Calloni 1889; [Isaia 2005]; [Rossi \& Bosio 2012]

Chorotype. - PAL.

MaCrOHABITAT. — Rocky lands, wetlands. 


\section{Araneus sturmi (Hahn, 1831)}

Literature. - De Angelis \& Fantoni 2008; [Rossi \& Bosio 2012].

Chorotype. - PAL.

MACROHABitAT. - Alpine pastures, broadleaved wood.

\section{Araniella cucurbitina (Clerck, 1757)}

NeW DaTA. — La Thuile: Comba Thuilette, 2000 m, wet meadow, 9.VII.2012, 2 \%, leg. Paschetta.

Literature. — Pavesi 1904; [Isaia 2005]; [Rossi \& Bosio 2012].

Chorotype. - PAL.

Macrohabitat. - Wetlands.

-Araniella opisthographa (Kulczyński, 1905)

Literature. — Rossi \& Bosio 2012.

Chorotype. - TUE.

MACROHABITAT. - Synanthropic habitats.

Notes. - See Tegenaria domestica.

\section{Argiope bruennichi (Scopoli, 1772)} (Fig. 6)

NEW DATA. — Pont-Saint-Martin: Natural Reserve of Stagno di Holay, 775 m, grove of reeds, 18.VIII.2011, 1 \&, leg. Paschetta \& Giuliano (MRSN-VDA); same locality, riparian vegetation, 13.VIII.2012, 10 , leg. Paschetta; Brissogne: Saint-Marcel, Quart, Nus: Natural Reserve of Les Îles, 530 m, grove of reeds, 17.VIII.2011, $20^{7}$, leg. Paschetta \& Giuliano.

Literature. — Rossi \& Arnò 1995; [Isaia 2005]; [Rossi \& Bosio 2012].

Chorotype. - PAL.

MACROHABITAT. - Riparian habitats, wetlands, shrublands.

\section{Cyclosa conica (Pallas, 1772)}

Literature. - Isaia 2005; [Rossi \& Bosio 2012].

Chorotype. - OlA.

MACROHABITAT. - Not specified.

Gibbaranea bituberculata (Walckenaer, 1802)

NeW DATA. - Sarre-Saint Pierre: Monte Torretta, 900 m, dry meadow, 24.V-13.VI.2012, 2 \%, leg. Negro (MRSN-VDA).

LiTERATURE. — Pesarini 1997; [Isaia 2005].

Chorotype. - PAL.
Macrohabitat. - Shrublands.

Notes. - See notes about Agelena labyrinthica.

*Hypsosinga albovittata (Westring, 1851)

NeW DATA. - Ayas: Antagnod, 1970-2070 m, alpine pasture, leg. Franco \& Negro.

CHOROTYPe. - SIE.

MACROHABITAT. - Alpine pastures.

NOTES. - New record for Aosta Valley.

•Hypsosinga heri (Hahn, 1831)

Literature. — Pesarini 1997; [Isaia 2005].

Chorotype. - PAL.

Macrohabitat. - Not specified.

Notes. - Not mentioned specifically for the region and not validated by new original data. The species has been reported for Aosta Valley on the base of the general record of Pesarini (1997), who considers the species widely distributed in Italy. The presence of this species in Val d'Aosta needs verification. The occurrence of the species in Aosta Valley is also considered doubtful by Rossi \& Bosio (2012).

\section{Hypsosinga pygmaea (Sundevall, 1831)}

LiterATURE. - Isaia 2005; [Rossi \& Bosio 2012].

Chorotype. - OLA.

Macrohabitat. - Not specified.

•Larinioides cornutus (Clerck, 1757)

Literature. — Pesarini 1997; [Isaia 2005].

Chorotype. - OLA.

Macrohabitat. - Not specified.

Notes. - See notes about Hypsosinga heri.

•Larinioides sclopetarius (Clerck, 1757)

Literature. - Rossi \& Bosio 2012.

Chorotype. - OLA.

MACROHABITAT. - Riparian habitats.

Notes. - See Tegenaria domestica. 


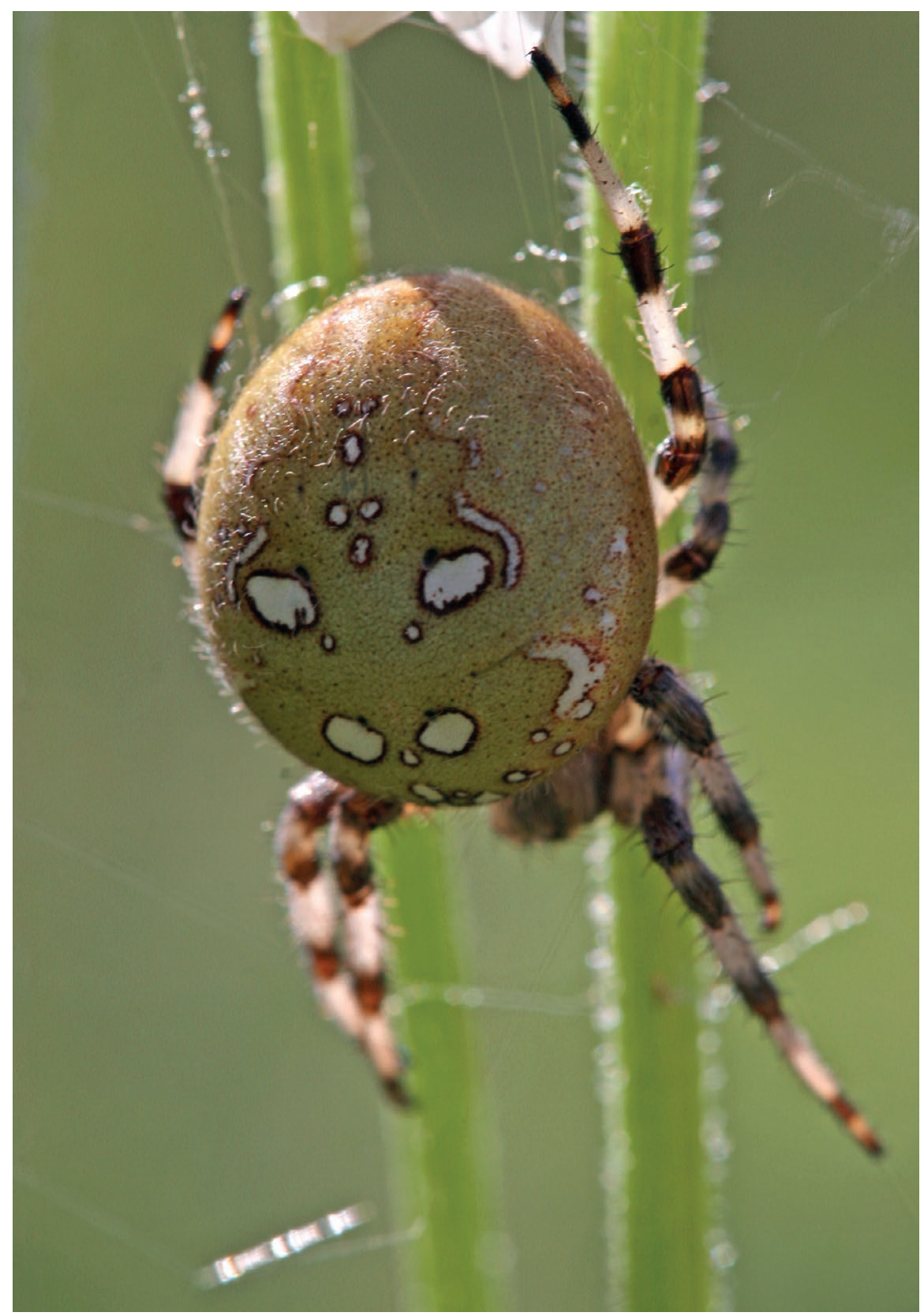

FIG. 5. - Araneus quadratus Clerck, 1757 (Araneidae), female. Average body length of the female: 10.5-20.5 mm. La Thuile: Comba Thuilette. Photograph: Mauro Paschetta 2008.

Mangora acalypha (Walckenaer, 1802)

NEW DATA. - La Thuile: Comba Thuilette, 2000 m, wet meadow, 17.VIII.2011, 1 ㅇ, leg. Paschetta \& Giuliano; Saint Denis: BrisonCly, 1000 m, broadleaved forest, 13.VI-4.VII.2012, 1 \&, leg. Negro; Pont-Saint-Martin: Natural Reserve of Stagno di Holay, 775 m, riparian vegetation, 13.VIII.2012, 2 \%, leg. Paschetta.

Literature. - Rossi \& Arnò 1995; [Isaia 2005]; [Rossi \& Bosio 2012].

Chorotype. - PAL.

MACROHABITAT. - Broadleaved forests, wetlands, shrublands, riparian habitats.
-Neoscona adianta (Walckenaer, 1802)

Literature. - Pesarini 1997; [Isaia 2005].

Chorotype. - PAL.

Macrohabitat. - Not specified.

Notes. - See notes about Hypsosinga heri.

- Nuctenea umbratica (Clerck, 1757)

Literature. — Pavesi 1904; [Isaia 2005]; [Rossi \& Bosio 2012].

CHOrotype. - SIE. 
MACROHABITAT. - Synantropic habitat.

Notes. - Old record in need of validation.

Parazygiella montana (C. L. Koch, 1834)

NEW DATA. - La Thuile: Comba Thuilette, 2000 m, rocky land, 17.VIII.2011, 1 ㅇ, leg. Paschetta \& Giuliano.

Literature. - Calloni 1889; [Isaia 2005]; Negro et al. 2009.

Chorotype. - SEU.

MACROHABITAT. - Rocky lands, alpine prairies.

\section{Singa hamata (Clerck, 1757)}

Literature. — Rossi \& Arnò 1995; [Isaia 2005]; [Rossi \& Bosio 2012].

Chorotype. - PAL.

MACROHABITAT. - Shrublands, broadleaved forests, wetlands.

Zilla diodia (Walckenaer, 1802)

Literature. - Isaia 2005; [Rossi \& Bosio 2012].

CHOrotype. - SIE.

MacrohabitaT. - Not specified.

\section{Zygiella x-notata (Clerck, 1757)}

Literature. - Isaia 2005; [Rossi \& Bosio 2012].

Chorotype. - OLA.

MACROHABITAT. - Not specified.

Family ATYPIDAE Thorell, 1870

Atypus affinis Eichwald, 1830

Literature. — De Angelis \& Fantoni 2008; [Rossi \& Bosio 2012].

Chorotype. - EUR.

MacrohabitaT. - Not specified.

*Atypus muralis Bertkau, 1890

New DATA. - Aymavilles: Pont d'Ael, 800 m, dry meadow, 24.V13.VI.2012, $10^{7}$, leg. Negro (MRSN-VDA).

Chorotype. - TUE.

MacrohabitaT. - Shrublands.

Notes. - New record for Aosta Valley.
Family ClubIONIDAE Wagner, 1887

Clubiona alpicola Kulczyński, 1882

Literature. — Negro et al. 2009; [Rossi \& Bosio 2012].

Chorotype. - TUE.

Macrohabitat. - Coniferous forests.

Clubiona brevipes Blackwall, 1841

Literature. - Isaia 2005; [Rossi \& Bosio 2012].

CHORotype. - PAL.

MacrohabitaT. - Not specified.

${ }^{*}$ Clubiona comta C. L. Koch, 1839

NEW DATA. - Brissogne: Saint-Marcel, Quart, Nus: Natural Reserve of Les îles, $530 \mathrm{~m}$, riparian vegetation, 29.VII-18.VIII.2011, 1 \%, leg. Paschetta \& Giuliano.

CHOrotype. - EUR.

MACROHABITAT. - Riparian habitats.

Notes. - New record for Aosta Valley.

${ }^{*}$ Clubiona corticalis (Walckenaer, 1802)

NEW DATA. - Gressan: Natural Reserve of Côte de Gargantua, 700 m, bradleaved wood, 24.V-13.VI.2012, 1 \%, leg. Negro; same locality, 13.VI-4.VII.2012, 1 \% , leg. Negro; Pont-Saint-Martin: Natural Reserve of Stagno di Holay, 775 m, wet meadow, 13.VI-4. VII.2011, 1 \&, leg. Paschetta \& Giuliano (MNHN); Saint Christophe: Tsatelet, $750 \mathrm{~m}$, bradleaved wood, 4-25.VII.2012, 1 운, leg. Negro (MRSN-VDA).

Chorotype. - TUE.

MACROHABITAT. - Broadleaved forests, wetlands.

Notes. - New record for Aosta Valley.

Clubiona diversa O. P.-Cambridge, 1862

NEW DATA. - Torgnon: Lo Ditor, 1950 m, peat bog, 26.VI-12. VII.2013, 1 o", leg. Negro (MRSN-VDA).

Literature. - Negro et al. 2009; [Rossi \& Bosio 2012].

CHorotype. - PAL.

MACROHABITAT. - Wetlands, alpine prairies. 


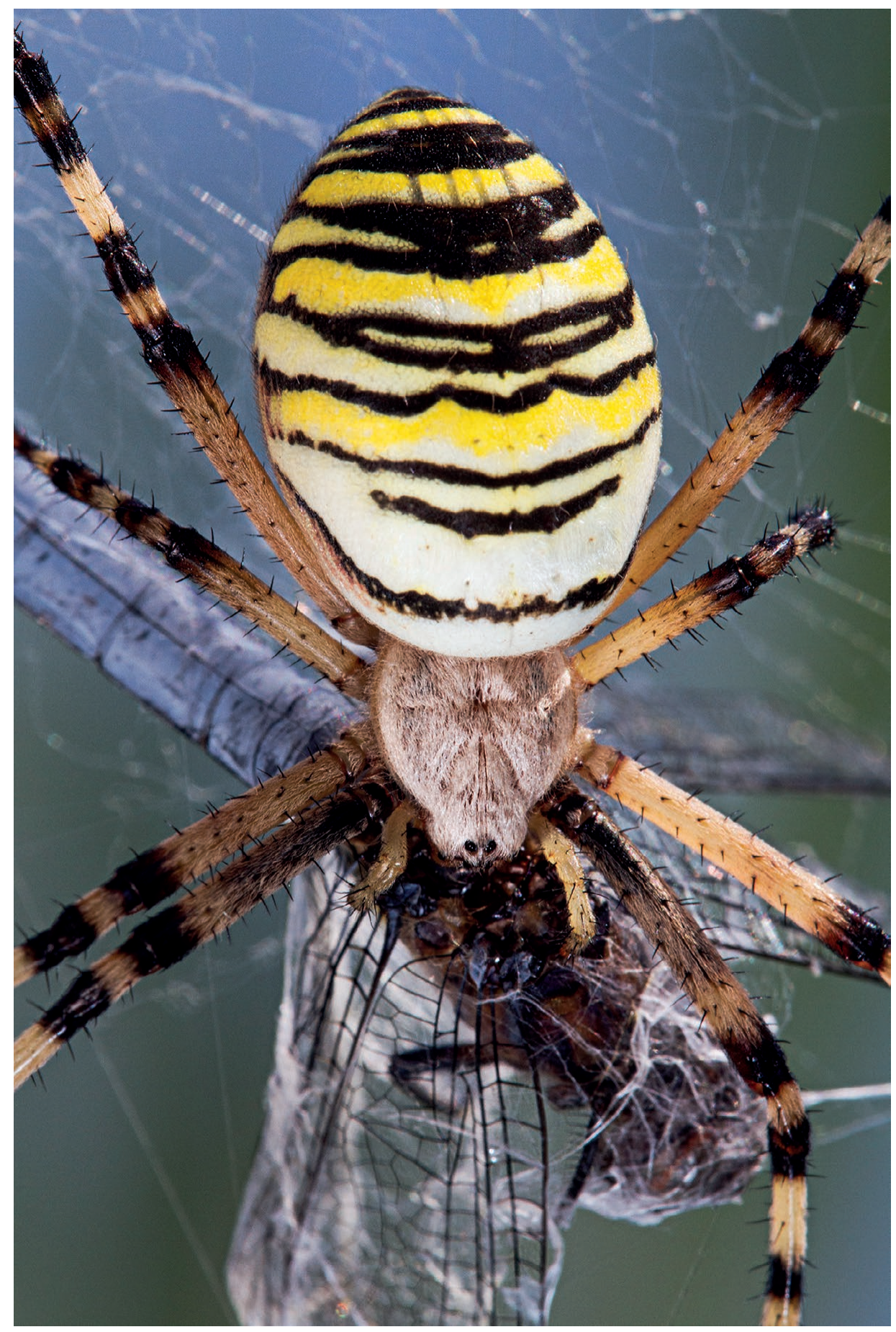

FIG. 6. - Argiope bruennichi (Scopoli, 1772) (Araneidae), female. Average body length of the female: 9.9-22 mm. Pont-Saint-Martin: Natural Reserve of Stagno di Holay. Photograph: Mauro Paschetta 2012.

Clubiona frutetorum L. Koch, 1867

Literature. — Isaia 2005; [Rossi \& Bosio 2012].

CHOROTYPe. - PAL.

Macrohabitat. - Not specified.
- Clubiona hilaris Simon, 1878

LiteratURE. - Simon 1878; Pavesi 1904; [Isaia 2005]; [Rossi \& Bosio 2012].

Chorotype. - SEU.

MACrohabitAT. - Coniferous forests. 


\section{Clubiona lutescens Westring, 1851}

LiterATURE. - Rossi \& Arnò 1995; [Isaia 2005]; [Rossi \& Bosio 2012].

Chorotype. - OLA.

Macrohabitat. - Wetlands.

Clubiona neglecta O. P.-Cambridge, 1862

NEW DATA. - Arvier: Lago di Lolair, 1175 m, wet meadow, 05-29. VII.2011, 1 o', leg. Paschetta \& Giuliano; Courmayeur: Val Ferret, $1740 \mathrm{~m}$, gravel bed of a river, 14-28.VIII.2013, 1 ơ, leg. Negro.

Literature. - Negro et al. 2009; [Rossi \& Bosio 2012].

Chorotype. - PAL.

MaCrOHABITAT. - Wetlands, gravel beds, riparian habitats, alpine prairies.

\section{Clubiona phragmitis C. L. Koch, 1843}

NEW DATA. - Pont-Saint-Martin: Natural Reserve of Stagno di Holay, 775 m, riparian vegetation, 05.IV.2012, 1 \%, leg. Isaia \& Paschetta; same locality, broadleaved forest (Castanea sativa), 26.VII14.VIII.2013, 1 \%, leg. Negro; Brissogne, Saint-Marcel, Quart, Nus: Natural Reserve of Les Îles, 526 m, riparian forest, 11-26.VI.2013, 1 ㅇ, leg. Negro; same locality, 26.VI-12.VII.2013, 1 ㅇ, leg. Negro (MRSN-VDA).

Literature. - Pesarini 1997; [Isaia 2005]; [Rossi \& Bosio 2012].

Chorotype. - PAL.

MACROHABITAT. - Riparian habitats, broadleaved forests.

Notes. - See notes about Agelena labyrinthica.

Clubiona reclusa O. P.-Cambridge, 1863

Literature. — Negro et al. 2009; [Rossi \& Bosio 2012].

Chorotype. - ASE.

MacrohabitAT. - Alpine prairies.

Clubiona stagnatilis Kulczyński, 1897

NeW DATA. — La Thuile: Comba Thuilette, 2000 m, peat bog, 17.VIII.2011, 1 \%, leg. Paschetta \& Giuliano (MRSN-VDA).

Literature. — Rossi \& Bosio 2012.

Chorotype. - PAL.

MacrohabitAT. - Wetlands.
Clubiona terrestris Westring, 1851

NEW DATA. - Torgnon: Lo Ditor, 1950 m, peat bog, 26.VI-12. VII.2013, 1 \% , leg. Negro; Morgex-La Salle: Natural Reserve of Marais, 896 m, grove of reeds, 26.VII-14.VIII.2013, 1 ㅇ, leg. Negro; Brissogne, Saint-Marcel, Quart, Nus: Natural Reserve of Les Illes, 526 m, riparian forest, 26.VII-14.VIII.2013, 1 o", leg. Negro; Saint Christophe: Tsatelet, $750 \mathrm{~m}$, broadleaved forest, 4.V-13. VI.2012, 1 \%, leg. Negro (MRSN-VDA); Challand-Saint-Victor: Natural Reserve of Lago di Villa, $820 \mathrm{~m}$, broadleaved forest, 26.VI12.VII.2013, 1 o", leg. Negro.

Literature. — De Angelis \& Fantoni 2008; [Rossi \& Bosio 2012].

CHOrotype. - EUR.

MACROHABITAT. - Wetlands, riparian habitats, broadleaved forests.

Family CyBaEIDAe Banks, 1892

-Argyroneta aquatica (Clerck, 1757)

Literature. - Rossi \& Bosio 2012.

Chorotype. - PAL.

MACROHABITAT. - Riparian habitats.

Notes. - See Tegenaria domestica.

Cybaeus intermedius Maurer, 1992

NeW DATA. - Pont-Saint-Martin: Natural Reserve of Stagno di Holay, $679 \mathrm{~m}$, broadleaved forest (Castanea sativa), 11-26.VI.2013, 18 o $^{7} 1$ \% , leg. Negro (MRSN-VDA); same locality, 26.VI-12. VII.2013, 2 o", leg. Negro.

Literature. - Negro et al. 2009; [Rossi \& Bosio 2012]; [Isaia \& Chiarle 2015].

CHOrotype. - AWNA.

MacrohabitaT. - Broadleaved forests, alpine prairies.

Family DictYNidAe O. P.-Cambridge, 1871

Dictyna arundinacea (Linnaeus, 1758)

NeW DATA. - La Thuile: Comba Thuilette, $2000 \mathrm{~m}$, peat bog, 10.VII.2012, 1 o 4 \% , leg. Paschetta, Giuliano, Mammola \& Marguerettaz; Morgex-La Salle: Natural Reserve of Marais, 900 m, wet meadow, 16.VIII.2011, 1 \%, leg. Paschetta \& Giuliano; same locality, grove of reeds, 9.VII.2012, 1 o, leg. Paschetta; same locality, riparian forest, 9.VII.2012, 2 \% leg. Paschetta.

Literature. — Rossi \& Arnò 1995; [Isaia 2005]; [Rossi \& Bosio 2012].

Chorotype. - OLA.

MACROHABITAT. - Wetlands, riparian habitats, broadleaved forests, shrublands. 
*Dictyna latens (Fabricius, 1775)

NEW DATA. — Morgex-La Salle: Natural Reserve of Marais, 900 m, wet meadows, 9.VII.2012, $10^{\prime \prime}$, leg. Paschetta; same locality, grove of reeds, 9.VII.2012, 2 \%, leg. Paschetta.

Chorotype. - SIE.

MACROHABITAT. - Wetlands.

Notes. - New record for Aosta Valley.

Family DysDeridae C. L. Koch, 1837

Dysdera crocata C. L. Koch, 1838

LiterATURE. - Isaia 2005; Negro et al. 2009; [Rossi \& Bosio 2012].

Chorotype. - COS.

MacrohabitaT. - Coniferous forest.

*Dysdera erythrina (Walckenaer, 1802)

NEW DATA. - Brissogne: Saint-Marcel, Quart, Nus: Natural Reserve of Les Îles, $530 \mathrm{~m}$, riparian forest, 26.VII-14.VIII.2013, 1 o" $^{7}$, leg. Negro; same locality, 29.VII-18.VIII.2011, 1 o", leg. Paschetta \& Giuliano; same locality, 14.VI-05.VII.2011, 1 \%, leg. Paschetta \& Giuliano; same locality, dry meadow, 5-29.VII.2011, 1 ơ, leg. Paschetta \& Giuliano.

Chorotype. - EUR.

MACROHABITAT. - Riparian habitats, shrublands.

Notes. - New record for Aosta Valley.

${ }^{\circ}$ Dysdera ninnii Canestrini, 1868

Literature. — Rossi \& Bosio 2012.

Chorotype. - SEU.

MACROHABITAT. - Broadleaved forests.

Notes. - According to Řezáč et al. (2014), D. ninnii is distributed in northeastern Italy, western Slovenia, northwestern Croatia. See also notes about Tegenaria domestica in the next section.

\section{Harpactea hombergi (Scopoli, 1763)}

NEW DATA. - Saint Denis: Brison-Cly, 1000 m, dry meadow, 13.VI-4.VII.2012, 1 ơ $^{\prime}$, leg. Negro (MNHN); Pont-Saint-Martin: Natural Reserve of Stagno di Holay, 679 m, broadleaved forest (Castanea sativa), 11-26.VI.2013, 4 o" 1 으, leg. Negro; same locality, 11-26.VI.2013, 1 \%, leg. Negro (MRSN-VDA); same locality, 26.VI-12.VII.2013, 1 o', leg. Negro; same locality, wet meadow, 5.IV.2012, 1 +, leg. Paschetta, Isaia; Challand-Saint-Victor: Natural Reserve of Lago di Villa, 820 m, wet meadow, 11-26.VI.2013, $10^{\text {" }}$, leg. Negro; same locality, broadleaved forest, 11-26.VI.2013, 1 0", leg. Negro, same locality, 26.VI-12.VII.2013, 2 o", leg. Negro; Saint Christophe: Tsatelet, $750 \mathrm{~m}$, broadleaved forest, 13.VI-4.VII.2012, $40^{\prime \prime}$, leg. Negro; same locality, 13.VI-4.VII.2012, $10^{\prime \prime}$, leg. Negro (MRSN-VDA); same locality 3-24.V.2012, 1 ơ, leg. Negro; same locality, 24.V-13.VI.2012, 1 ơ, leg. Negro.

Literature. - Isaia 2000, [2005]; De Angelis \& Fantoni 2008; [Rossi \& Bosio 2012].

CHOROTYPe. - EUR.

MACrOHABITAT. - Shrublands, broadleaved forests, wetlands, alpine grasslands.

Harpactocrates drassoides (Simon, 1882)

NEW DATA. - Arvier: Natural Reserve of Lago di Lolair, 1175 m, broadleaved forest, 24.V-13.VI.2012, $10^{7}$, leg. Negro (MNHN); same locality, 3-24.V.2012, 2 o", leg. Negro (MRSN-VDA).

Literature. — De Angelis \& Fantoni 2008; [Rossi \& Bosio 2012].

CHOROTyPe. - ALPW.

MACROHABITAT. - Broadleaved forests.

Family ERESIDAE C. L. Koch, 1845

Eresus kollari Rossi, 1846

Literature. - Isaia 2005; [Rossi \& Bosio 2012].

CHOrotype. - SIE.

MACROHABITAT. - Not specified.

Family EUTICHURIDAE Lehtinen, 1967

Cheiracanthium elegans Thorell, 1875

Literature. - Isaia 2005; [Rossi \& Bosio 2012].

Chorotype. - ASE.

MACROHABITAT. - Not specified.

Cheiracanthium mildei L. Koch, 1864

NEW DATA. - Challand-Saint-Victor: Natural Reserve of Lago di Villa, $820 \mathrm{~m}$, broadleaved forest, 26.VII-14.VIII.2013, $1 \mathrm{O}^{\text {", }}$ leg. Negro.

Literature. - Pavesi 1904; [Isaia 2005]; [Rossi \& Bosio 2012].

CHOrotype. - OLA.

MACROHABITAT. - Synanthropic habitats, broadleaved forests.

•Cheiracanthium oncognathum Thorell, 1871

Literature. — Rossi \& Bosio 2012.

Chorotype. - PAL. 
MACROHABITAT. - Riparian habitats.

Notes. - See Tegenaria domestica.

\section{Cheiracanthium punctorium (Villers, 1789)}

NeW DATA. - La Thuile: Comba Thuilette, 2000 m, riparian vegetation, 17.VIII.2011, $10^{7} 1$ o, leg. Paschetta \& Giuliano; Brissogne, Saint-Marcel, Quart, Nus: Natural Reserve of Les Îles, $530 \mathrm{~m}$, dry meadow, 17.VIII.2011, 2 \%, leg. Paschetta \& Giuliano (MRSN-VDA).

LiterATURE. - Rossi \& Bosio 2012.

Chorotype. - TUE.

MACROHABITAT. - Riparian habitats, shrublands.

Family FiLiSTATIDAE Ausserer, 1867

Filistata insidiatrix (Forsskål, 1775)

Literature. - Isaia 2005; [Rossi \& Bosio 2012].

Chorotype. - MED.

MACROHABITAT. - Not specified.

\section{Family GNAPHOSIDAE Pocock, 1898}

\section{Aphantaulax trifasciata (O. P.-Cambridge, 1872)}

Literature. - Negro et al. 2009; [Rossi \& Bosio 2012].

Chorotype. - PAL.

MACROHABITAT. - Coniferous forests.

\section{Berlandina nubivaga (Simon, 1878)}

LiterATURE. - Isaia et al. 2009; Negro et al. 2009; [Rossi \& Bosio 2012].

Chorotype. - ALPW.

MacrohabitAT. - Alpine pastures, coniferous forests.

\section{Callilepis nocturna (Linnaeus, 1758)}

NEW DATA. - Morgex-La Salle: Natural Reserve of Natural Reserve of Marais, $896 \mathrm{~m}$, grove of reeds, 11-26.VI.2013, $9 \mathrm{o}^{7} 1$ \%, leg. Negro; same locality, grove of reeds, 12-26.VII.2013, 1 \% leg. Negro; same locality, 26.VII-14.VIII.2013, 2 이, leg. Negro; Challand-Saint-Victor: Natural Reserve of Lago di Villa, $820 \mathrm{~m}$, meadow, 11-26.VI.2013, 1 ơ, leg. Negro; Saint Christophe: Tsatelet, $750 \mathrm{~m}$, broadleaved forest, 13.VI-4.VII.2012, 1 o", leg. Negro; Aymavilles: Pont d'Ael, 800 m, broadleaved forest, 4-25.VII.2012, 1 \%, leg. Negro; same locality, dry meadow, 24.V-13.VI.2012, 1 ㅇ, leg. Negro (MRSN-VDA); Ayas: Antagnod, 1970-2070 m, alpine grassland, 2007, leg. Franco \& Negro.
Literature. - Isaia 2005; De Angelis \& Fantoni 2008; Negro et al. 2009; [Rossi \& Bosio 2012].

Chorotype. - PAL.

MACROHABITAT. - Wetlands, alpine grasslands, broadleaved forests, shrublands, synanthropic habitats.

-Civizelotes gracilis (Canestrini, 1868)

LiterATURE. - Rossi \& Bosio 2012.

Chorotype. - SEU.

MACROHABITAT. - Broadleaved forests.

Notes. - See Tegenaria domestica.

\section{- Drassodes fugax (Simon, 1878)}

Literature. - Pavesi 1904; di Caporiacco 1928; [Isaia 2005]; [Rossi \& Bosio 2012].

Chorotype. - PAL.

MACROHABITAT. - Alpine prairies.

Notes. - The presence of this species in Aosta Valley needs to be verified in light of the revision of the pubescens group by Hervé \& Rollard (2009).

\section{Drassodes lapidosus (Walckenaer, 1802)}

New Data. - Aymavilles: Pont d'Ael, 800 m, dry meadow, 24.V-13.VI.2012, 8 o 11 \%, leg. Negro; same locality, 13.VI-4. VII.2012, $10^{\text {"7 }} 6$ \%, leg. Negro; same locality, 4-25.VII.2012, $10^{\text {"7 }}$ 3 ㅇ, leg. Negro; same locality, 25.VII-20.VIII.2012, 2 이, leg. Negro; same locality, broadleaved forest, 13.VI-4.VII.2012, 3 \% , leg. Negro; same locality, 25.VII-20.VIII.2012, 1 \% , leg. Negro; Morgex-La Salle: Natural Reserve of Marais, 896 m, riparian forest, 26.VI-12.VII.2013, 1 o', leg. Negro; Sarre-Saint Pierre: Monte Torretta, $900 \mathrm{~m}$, broadleaved forest, 4-25.VII.2012, 1 운, leg. Negro; same locality, 24.V-13.VI.2012, 1 이, leg. Negro; same locality, drey meadow, 3-24.V.2012, 1 o", leg. Negro; same locality, 24.V-13.VI.2012, $40^{7} 6$ o , leg. Negro; same locality, 13.VI-4.VII.2012, $1 \mathrm{O}^{7} 1$ ㅇ, leg. Negro; same locality, 4-25.VII.2012, 1 o' 6 9 , leg. Negro; same locality, 25.VII-20. VIII.2012, 1 ㅇ, leg. Negro; Verrayes: stagno di Loson, 1520 m, grove of reeds/peat bog, 13.VI-4.VII.2011, 1 \%, leg. Paschetta \& Giuliano; Arvier: Lago di Lolair, 1175 m, wet meadow, 5-29.VII.2011, 1 o", leg. Paschetta \& Giuliano; same locality, broadleaved forest, 24.V-13.VI.2012, 1 \%, leg. Negro; same locality, 25.VII-20.VIII.2012, 1 \%, leg. Negro; Courmayeur: Val Ferret, $1740 \mathrm{~m}$, gravel bed of a river, 14-28.VIII.2013, $1 \mathrm{o}^{7}$, leg. Negro; Challand-Saint-Victor: Natural Reserve of Lago di Villa, $820 \mathrm{~m}$, wet meadow, 13.VI-4.VII.2011, $1 \mathrm{o}^{\text {" }}$, leg. Paschetta \& Giuliano; same locality, 4-28.VII.2011, 1 ㅇ, leg. Paschetta \& Giuliano; same locality, 28.VII-17.VIII.2011, $1 \%$, leg. Paschetta \& Giuliano; same locality, 13.VIII.2012, 1 \%, leg. Paschetta; same locality, 11-26.VI.2013, $20^{\prime \prime} 1 \%$, leg. Negro; same locality, 26.VI-12.VII.2013, $20^{\prime \prime}$, leg. Negro; same locality, 12-26.VII.2013, 2 \%, leg.Negro; same locality, 26.VI-12.VII.2013, 1 or $^{4}$ \% , leg. Negro; same locality, 12-26.VII.2013, 1 ㅇ, leg. Negro; same locality, 26.VII-14. 
VIII.2013, 1 ㅇ, leg. Negro; same locality, broadleaved forest, 26.VI-12.VII.2013, 1 o' 2 \% , leg. Negro; same locality, 12-26. VII.2013, 2 \% , leg. Negro; same locality, 26.VII-14.VIII.2013, 1 o, leg. Negro; same locality, 14-28.VIII.2013, 1 ㅇ, leg. Negro same locality, 28.VIII-16.IX.2013, 1 \%, leg. Negro; Saint Christophe: Tsatelet, $750 \mathrm{~m}$, broadleaved forest, 3-24.V.2012, 1 ㅇ, leg. Negro; same locality, 24.V-13.VI.2012, 3 ơ 3 \%, leg. Negro; same locality, 24.V-13.VI.2012, leg. Negro; same locality, 13.VI-4.VII.2012, 1 \%, leg. Negro; Brissogne, SaintMarcel, Quart, Nus: Natural Reserve of Les Îles, 530 m, dry meadow, 14.VI-5.VII.2011, 1 일. leg. Paschetta \& Giuliano; same locality, 5-29.VII.2011, 1 o, leg. Paschetta \& Giuliano; same locality, 5-29.VII.2011, 3 \%, leg. Paschetta \& Giuliano; same locality, 29.VII-18.VIII.2011, 1 o, leg. Paschetta \& Giuliano; same locality, 18.VIII-16.IX.2011, 1 \% , leg. Paschetta $\&$ Giuliano; same locality, 26.VII-14.VIII.2013, 2 \% , leg. Negro; same locality, riparian forest, 11-26.VI.2013, 1 \%, leg. Negro; Saint Denis: Brison-Cly, 1000 m, broadleaved forest, 24.V-13. VI.2012, $10^{7} 4$ o , leg. Negro; same locality, 13.VI-4.VII.2012, $10^{7} 1$ o, leg. Negro; same locality, 4-25.VII.2012, $10^{\text {7 }} 1$ 우, leg. Negro; same locality, dry meadow, 24.V-13.VI.2012, $10^{\prime \prime}$, leg. Negro; same locality, 4-25.VII.2012, 1 ơ 1 \& , leg. Negro; Pont-Saint-Martin: Natural Reserve of Stagno di Holay, 775 m, riparian vegetation, 13.VI-4.VII.2011, $20^{7}$, leg. Paschetta \& Giuliano; same locality, wet meadow, 13.VI-4.VII.2011, $20^{\circ}$, leg. Paschetta \& Giuliano; same locality, 13.VI-4.VII.2011, $1 \sigma^{7}$, leg. Paschetta \& Giuliano (MRSN-VDA); same locality, 4-28.VII.2011, $10^{*} 1$ \%, leg. Paschetta \& Giuliano; same locality, 28.VII-17.VIII.2011, 1 \% , leg. Paschetta \& Giuliano; same locality, 18.VIII-15.IX.2011, 1 ㅇ, leg. Paschetta \& Giuliano; same locality, 26.VI-12.VII.2013, 1 o", leg. Negro; same locality, 12-26.VII.2013, 1 o $^{7} 1$ \%, leg. Negro; same locality, 26.VII-14.VIII.2013, 1 ㅇ, leg. Negro; same locality, 14-28. VIII.2013, 1 ㅇ, leg. Negro; same locality, 28.VIII-16.IX.2013, 1 , leg. Negro; same locality, broadleaved forest (Castanea sativa), 11-26.VI.2013, 1 o", leg. Negro; same locality, 26.VI-12. VII.2013, 4 ㅇ, leg. Negro; same locality, 12-26.VII.2013, 1 \%, leg. Negro; same locality, 26.VII-14.VIII.2013, 1 \&, leg. Negro; Gressan: Natural Reserve of Côte de Gargantua, $700 \mathrm{~m}$, dry meadow, 3-24.V.2012, 1 o", leg. Negro; same locality, 4-25. VII.2012, 1 \%, leg. Negro; same locality, 25.VII-20.VIII.2012, 1 \%, leg. Negro; same locality, broadleaved forest, 24.V-13. VI.2012, 1 o, leg. Negro; same locality, 13.VI-4.VII.2012, 1 \%, leg. Negro; same locality, 4-25.VII.2012, 1 \&, leg. Negro; same locality, 25.VII-20.VIII.2012, 1 \%, leg. Negro; Torgnon: Lo Ditor, 1950 m, peat bog, 1-26.VI.2013, $90^{\circ}$, leg. Negro; same locality, 26.VI-12.VII.2013, 6 ơ 6 \% , leg. Negro; same locality, 12-26.VII.2013, $10^{7} 1$ \% , leg. Negro; same locality, 26.VII-14.VIII.2013, 3 ơ, leg. Negro; La Thuile: Comba Thuilette, $2000 \mathrm{~m}$, rocky land, 13.IX.2012, 2 \%, leg. Paschetta, Mammola \& Marguerettaz; Ayas: Antagnod, 1970-2070 m, ski pistes, 2007, leg. Franco \& Negro; same locality, alpine prairies, 2007, leg. Franco \& Negro; Ayas: Champoluc, 1650-1850 m, ski pistes, 2007, leg. Franco \& Negro.

Literature. - Calloni 1889; di Caporiacco 1928; Isaia 2000, [2005]; De Angelis \& Fantoni 2008; Negro et al. 2009, 2010, 2013; Gobbi et al. 2010; [Rossi \& Bosio 2012].

Chorotype. - PAL.

MacrohabitaT. - Shrublands, riparian habitats, gravel beds, broadleaved forests, coniferous forests, wetlands, alpine grasslands, alpine prairies, ski pistes, rocky lands, synanthropic habitats.

Notes. - Citations of D. cupreus (Blackwall, 1834) are here included under D. lapidosus according to Bolzern \& Hänggi (2006).
${ }^{\circ}$ Drassodes luteomicans (Simon, 1878)

LiteraTuRE. — Di Caporiacco 1928; [Isaia 2005]; [Rossi \& Bosio 2012].

CHOrotype. - SEU.

Macrohabitat. - Alpine prairies.

Notes. - According to Pantini \& Isaia (2015) all citations from the Italian peninsula, including di Caporiacco (1928), need confirmation.

\section{Drassodes pubescens (Thorell, 1856)}

NEW DATA. - Brissogne, Saint-Marcel, Quart, Nus: Natural Reserve of Les Îles, 530 m, dry meadow, 14.VI-5.VII.2011, $30^{\text {" }}$ 1 o, leg. Paschetta \& Giuliano; same locality, 5-29.VII.2011, 3 \% leg. Paschetta \& Giuliano, same locality, 29.VII-18.VIII.2011, 1 ㅇ, leg. Paschetta \& Giuliano; same locality, 18.VIII-16.IX.2011, 3 \%, leg. Paschetta \& Giuliano; same locality, riparian vegetation, 5-29. VII.2011, 1 \%, leg. Paschetta \& Giuliano; Morgex-La Salle: Natural Reserve of Marais, 896 m, grove of reeds, 11-26.VI.2013, 1 o", leg. Negro; same locality, 26.VII-14.VIII.2013, 1 \%, leg. Negro; same locality, riparian forest, 11-26.VI.2013, 1 o', leg. Negro; same locality, 26.VI-12.VII.2013, $10^{7} 1$ \%, leg. Negro; same locality, riparian forest, 28.VIII-16.IX.2013, 1 \% , leg. Negro; Aymavilles: Pont d'Ael, 800 m, dry meadow, 24.V-13.VI.2012, 2 o', leg. Negro; same locality, 13.VI-4.VII.2012, $20^{7} 4$ o, leg. Negro; same locality, 800 m, broadleaved forest, 25.VII-20.VIII.2012, 1 ㅇ, leg. Negro; Saint Christophe: Tsatelet, $750 \mathrm{~m}$, broadleaved forest, 13.VI-4.VII.2012, $10^{7} 1$ \%, leg. Negro; Courmayeur: Val Ferret, 1630 m, shrubland, 26.VII-14.VIII.2013, 1 \% , leg. Negro; Torgnon: Lo Ditor, 1950 m, peat bog, 11-26.VI.2013, 1 ơ 1 o, leg. Negro; same locality, 12-26. VII.2013, 1 o', leg. Negro; Pont-Saint-Martin: Natural Reserve of Stagno di Holay, 616 m, alpine grassland, 26.VI-12.VII.2013, $10^{\prime \prime}$, leg. Negro; Arvier: Lago di Lolair, $1175 \mathrm{~m}$, wet meadow, 14.VI-5. VII.2011, $30^{7}$, leg. Paschetta \& Giuliano; same locality, 29.VII18.VIII.2011, 1 \% leg. Paschetta \& Giuliano; Verrayes: stagno di Loson, $1520 \mathrm{~m}$, grove of reeds/peat bog, 13.VI-4.VII.2011, $20^{7}$, leg. Paschetta \& Giuliano; same locality, 13.VI-4.VII.2011, $10^{\prime \prime}$, leg. Paschetta \& Giuliano (MRSN-VDA); same locality, 4-28. VII.2011, $10^{7}$, leg. Paschetta \& Giuliano; same locality, 28.VII-17. VIII.2011, 1 ᄋ, leg. Paschetta \& Giuliano; Ayas: Champoluc, 16501850 m, ski pistes, 2007, leg. Franco \& Negro; Ayas: Antagnod, 1970-2070 m, alpine pastures, leg. Franco \& Negro.

Literature. - De Angelis \& Fantoni 2008; Negro et al. 2009; [Rossi \& Bosio 2012].

CHOROTYPe. - PAL.

MACROHABITAT. - Wetlands, broadleaved forests, shrublands, riparian habitats, alpine pastures, ski pistes, alpine grasslands.

\section{Drassodes villosus (Thorell, 1856)}

LiteraTURE. - Calloni 1889; [Isaia 2005]; De Angelis \& Fantoni 2008; [Rossi \& Bosio 2012].

CHORotype. - PAL.

MaCrohabitaT. - Not specified. 
Drassodex drescoi Hervé, Roberts \& Murphy, 2009

LiterATURE. - Gobbi et al. 2010; Negro et al. 2010.

Chorotype. - SEU.

MACROHABITAT. - Rocky lands.

${ }^{\circ}$ Drassodex heeri (Pavesi, 1873)

LiterATURE. — Di Caporiacco 1928; [Isaia 2005]; [Rossi \& Bosio 2012].

Chorotype. - SEU.

MACROHABITAT. - Rocky lands.

Notes. - It seems very likely that the record by di Caporiacco (1928) refers to $D$. drescoi, distributed in North-Western Alps. Unlikely, D. heeri is a Central Alpine species (Hervé et al. 2009; Hänggi \& Stäubli 2012).

\section{${ }^{\circ}$ Drassodex hispanus (L. Koch, 1866)}

Literature. - Calloni 1889; Pavesi 1904; [Isaia 2005]; [Rossi \& Bosio 2012].

Chorotype. - EUR.

MacrohabitaT. - Not specified.

Notes. - According to Hervé et al. (2009) data before 2009 are not reliable. The presence in Italy is also doubtful according to Pantini \& Isaia (2015). Older records of D. hispanus possibly refer to $D$. lesserti. The occurrence of this species in Aosta Valley was also considered uncertain by Rossi \& Bosio (2012).

\section{Drassyllus praeficus (L. Koch, 1866)}

NEW DATA. - Pont-Saint-Martin: Natural Reserve of Stagno di Holay, 775 m, wet meadow, 18.VIII-15.IX.2011, 1 \%, leg. Paschetta \& Giuliano (MRSN-VDA); same locality, meadow, 11-26. VI.2013, $10^{7}$, leg. Negro; Challand-Saint-Victor: Natural Reserve of Lago di Villa, 820 m, meadow, 11-26.VI.2013, 1 \%, leg. Negro; Ayas: Antagnod, 1970-2070 m, ski pistes, leg. Franco \& Negro.

Literature. - Isaia 2005; De Angelis \& Fantoni 2008; Negro et al. 2009; [Rossi \& Bosio 2012].

Chorotype. - SIE.

MACROHABITAT. - Alpine grasslands, wetlands, alpine pastures, ski pistes.

\section{Drassyllus pumilus (C. L. Koch, 1839)}

NEW DATA. - Morgex-La Salle: Natural Reserve of Marais, 900 m, wet meadow, 14.VI-5.VII.2011, 2 ơ 1 ९, leg. Paschetta \& Giuliano; same locality, riparian forest, 14.VI-5.VII.2011, 1 우 leg. Paschetta \& Giuliano; Challand-Saint-Victor: Natural Reserve of Lago di Villa, 820 m, wet meadow, 28.VII-17.VIII.2011, 1 \% leg. Paschetta \& Giuliano; same locality, 13.VI-4.VII.2011, $10^{\prime \prime}$ $2 \%$, leg. Paschetta \& Giuliano; Brissogne, Saint-Marcel, Quart, Nus: Natural Reserve of Les Îles, 526 m, dry meadow, 1-26.
VI.2013, 2 9, leg. Negro; Verrayes: stagno di Loson, 1520 m, grove of reeds/peat bog, 28.VII-17.VIII.2011, 1 ㅇ, leg. Paschetta \& Giuliano (MRSN-VDA); Pont-Saint-Martin: Natural Reserve of Stagno di Holay, 775 m, wet meadow, 28.VII-17.VIII.2011, 1 \%, leg. Paschetta \& Giuliano (MNHN).

LiTERATURE. - Isaia 2005; [Rossi \& Bosio 2012].

Chorotype. - EUR.

MACROHABITAT. - Wetlands, shrublands, riparian habitats.

\section{Drassyllus pusillus (C. L. Koch, 1833)}

NEW DATA. - Torgnon: Lo Ditor, 1950 m, peat bog, 26.VI-12. VII.2013, 1 0", leg. Negro; same locality, 26.VII-14.VIII.2013, 3 ㅇ, leg. Negro; Brissogne, Saint-Marcel, Quart, Nus: Natural Reserve of Les Îles, 530 m, dry meadow, 14.VI-5.VII.2011, 3 \%, leg. Paschetta \& Giulian; same locality, 5-29.VII.2011, 1 ᄋ, leg. Paschetta \& Giuliano; Ayas: Antagnod, 1970-2070 m, ski pistes, 2007, leg. Franco \& Negro.

Literature. - Isaia 2005; De Angelis \& Fantoni 2008; Negro et al. 2009; [Rossi \& Bosio 2012].

Chorotype. - PAL.

MACROHABITAT. - Wetlands, shrublands, ski pistes, alpine pastures.

\section{*Drassyllus villicus (Thorell, 1875)}

NEW DATA. - Challand-Saint-Victor: Natural Reserve of Lago di Villa, 820 m, wet meadow, 13.VI-4.VII.2011,3 9 , leg. Paschetta \& Giuliano; same locality, 13.VI-4.VII.2011, 1 \% , leg. Paschetta \& Giuliano (MRSN-VDA); same locality, broadleaved forest, 11-26. VI.2013, $10^{7}$, leg. Negro (MRSN-VDA); same locality, 26.VI-12. VII.2013, 1 \% , leg. Negro; same locality, 12-26.VII.2013, 1 \% leg. Negro; Saint Christophe: Tsatelet, $750 \mathrm{~m}$, broadleaved forest, 24.V-13.VI.2012, $10^{7}$, leg. Negro (MRSN-VDA); same locality, 4-25.VII.2012, 1 \%, leg. Negro; same locality, 24.V-13.VI.2012, $1 \%$, leg. Negro (MRSN-VDA); same locality, 4-25.VII.2012, 1 \%, leg. Negro (MNHN); Sarre-Saint Pierre: Monte Torretta, 900 m, broadleaved forest, 24.V-13.VI.2012, 2 o", leg. Negro.

CHOrotype. - EUR.

MACROHABITAT. - Broadleaved forests, wetlands.

Notes. - New record for Aosta Valley.

\section{Echemus angustifrons (Westring, 1861)}

NEW DATA. - Challand-Saint-Victor: Natural Reserve of Lago di Villa, 820 m, wet meadow, 13.VI-4.VII.2011, 1 ơ, leg. Paschetta \& Giuliano (MRSN-VDA).

Literature. — De Angelis \& Fantoni 2008; [Rossi \& Bosio 2012].

Chorotype. - ASE.

MACROHABITAT. - Wetlands. 


\section{-Gnaphosa alpica Simon, 1878}

Literature. - Di Caporiacco 1928; [Isaia 2005]; [Rossi \& Bosio 2012].

Chorotype. - SEU.

Macrohabitat. - Alpine prairies.

Notes. - Old record in need of validation. See notes on this species in Pantini \& Isaia (2015)

\section{Gnaphosa badia (L. Koch, 1866)}

NEW DATA. - La Thuile: Comba Thuilette, $2000 \mathrm{~m}$, rocky land, 10.VII.2012, 1 ㅇ, leg. Paschetta, Giuliano, Mammola \& Marguerettaz; Ayas: Antagnod, 1970-2070 m, ski pistes, leg. Franco \& Negro.

Literature. - Calloni 1889; Pavesi 1904; di Caporiacco 1928; Grimm 1985; [Isaia 2005]; De Angelis \& Fantoni 2008; Negro et al. 2009, 2013; Gobbi et al. 2010; [Rossi \& Bosio 2012].

CHOROTYPe. - SIE.

MACROHABITAT. - Ski pistes, coniferous forests, rocky lands.

\section{Gnaphosa bicolor (Hahn, 1833)}

NEW DATA. - Arvier: Lago di Lolair, $1175 \mathrm{~m}$, broadleaved forest, 4-25.VII.2012, 1 ㅇ, leg. Negro (MRSN-VDA).

LiterATURE. — De Angelis \& Fantoni 2008; [Rossi \& Bosio 2012].

ChOrotype. - SIE.

MACROHABitaT. - Broadleaved forests.

\section{- Gnaphosa leporina (L. Koch, 1866)}

Literature. - Di Caporiacco 1928; [Isaia 2005]; [Rossi \& Bosio 2012].

Chorotype. - PAL.

MacrohabitaT. - Not specified.

NOTES. - Old record in need of validation.

\section{${ }^{*}$ Gnaphosa lucifuga (Walckenaer, 1802)}

NEW DATA. - Torgnon: Lo Ditor, 1950 m, peat bog, 26.VI-12. VII.2013, 1 ㅇ, leg. Negro (MRSN-VDA).

Chorotype. - PAL.

MACROHABITAT. - Wetlands.

Notes. - New record for Aosta Valley.
VII.2012, 1 o', leg. Negro; Pont-Saint-Martin: Natural Reserve of Stagno di Holay, 775 m, wet meadow, 13.VI-4.VII.2011, 1 ơ 1 \%, leg. Paschetta \& Giuliano (MNHN); same locality, 4-28.VII.2011, $3 \$$, leg. Paschetta \& Giuliano; same locality, 28.VII-17.VIII.2011, 2 \%, leg. Paschetta \& Giuliano; same locality, meadow, 11-26. VI.2013, $60^{7} 3$ \% , leg. Negro; same locality, 12-26.VII.2013, 1 o", $^{\prime \prime}$ leg. Negro; same locality, broadleaved forest (Castanea sativa), 1126.VI.2013, $10^{7}$, leg. Negro; Sarre-Saint Pierre: Monte Torretta, 900 m, dry meadow, 3-24.V.2012, 1 o', leg. Negro; same locality, 24.V-13.VI.2012, 1 ơ $^{7}$, leg. Negro; same locality, 13.VI-4.VII.2012, $10^{7} 1$ ㅇ, leg. Negro; same locality, 4-25.VII.2012, 1 \% , leg. Negro (MRSN-VDA); same locality, 25.VII-20.VIII.2012, 2 \%, leg. Negro; same locality, broadleaved forest, 24.V-13.VI.2012, 1 o', leg. Negro; Challand-Saint-Victor: Natural Reserve of Lago di Villa, 820 m, wet meadow, 11-26.VI.2013, 1 \%, leg. Negro; same locality, 26.VI12.VII.2013, 1 o", leg. Negro; Aymavilles: Pont d'Ael, 800 m, dry meadow, 13.VI-4.VII.2012, $10^{7}$, leg. Negro; Saint Denis: BrisonCly, 1000 m, broadleaved forest, 24.V-13.VI.2012, 3 o", leg. Negro; same locality, 13.VI-4.VII.2012, 1 \% , leg. Negro; same locality, dry meadow, 4-25.VII.2012, 1 \%, leg. Negro; same locality, 13.VI-4. VII.2012, 2 o $^{7} 1$ \%, leg. Negro; same locality, 4-25.VII.2012, $10^{7}$ 1 ㅇ, leg. Negro.

Literature. - Simon 1878; Pavesi 1904; di Caporiacco 1928; [Isaia 2005]; [Rossi \& Bosio 2012].

CHOROTYPe. - SIE.

MACROHABITAT. - Broadleaved forests, alpine grasslands, shrublands, wetlands.

\section{Gnaphosa montana (L. Koch, 1866)}

NEW DATA. - Arvier: Lago di Lolair, $1175 \mathrm{~m}$, broadleaved forest, 24.V-13.VI.2012, $10^{\prime}$, leg. Negro (MRSN-VDA); Saint Denis: Brison-Cly, 1000 m, dry meadow, 24.V-13.VI.2012, 2 o", leg. Negro (MNHN).

Literature. - Di Caporiacco 1928; [Isaia 2005].

CHOrotype. - SIE.

MACROHABITAT. - Broadleaved forests, shrublands.

\section{Gnaphosa muscorum (L. Koch, 1866)}

NeW DATA. - Aymavilles: Pont d'Ael, 800 m, dry meadow, 24.V13.VI.2012, 1 ơ, leg. Negro (MRSN-VDA); same locality, 24.V-13. VI.2012, $20^{7}$, leg. Negro (MRSN-VDA); same locality, 25.VII20.VIII.2012, 1 क , leg. Negro (MNHN); same locality, 13.VI-4. VII.2012, 3 o", leg. Negro; same locality, 13.VI-4.VII.2012, 1 o", leg. Negro (MNHN); Sarre-Saint Pierre: Monte Torretta, 900 m, dry meadow, 24.V-13.VI.2012, 1 \%, leg. Negro.

LiTERATURE. - Calloni 1889; Grimm 1985; Isaia 2000, [2005]; [Rossi \& Bosio 2012].

Chorotype. - OLA.

MACROHABITAT. - Shrublands, rocky lands.
NEW DATA. - Arvier: Lago di Lolair, 1175 m, broadleaved forest, 13.VI-4.VII.2012, 1 o 1 ㅇ, leg. Negro; same locality, 4-25.
-Gnaphosa occidentalis Simon, 1878

LiTERATURE. - Di Caporiacco 1928; [Isaia 2005]; [Rossi \& Bosio 2012]. 
CHOrotype. - EUR.

MacrohabitaT. - Alpine prairies.

Notes. - Old record in need of validation.

\section{Gnaphosa petrobia L. Koch, 1872}

Literature. - Calloni 1889; Pavesi 1904; di Caporiacco 1928; [Isaia 2005]; Negro et al. 2010, 2013; [Rossi \& Bosio 2012].

Chorotype. - SEU.

Macrohabitat. - Alpine prairies, ski pistes.

\section{- Gnaphosa tigrina Simon, 1878}

LiterATURE. - Calloni 1889; [Isaia 2005].

Chorotype. - WME.

Macrohabitat. - Not specified.

Notes. - Old record in need of validation. The occurrence of the species in Aosta Valley is also considered doubtful by Rossi \& Bosio (2012).

\section{Haplodrassus aenus Thaler, 1984}

NEW DATA. - Ayas: Antagnod, 1970-2070 m, ski pistes, 2007, leg. Franco \& Negro.

Literature. — Negro et al. 2009; [Rossi \& Bosio 2012].

Chorotype. - ALP.

MACROHABITAT. - Ski pistes.

\section{•Haplodrassus cognatus (Westring, 1861)}

LiterATURE. - Di Caporiacco 1928; [Isaia 2005]; [Rossi \& Bosio 2012].

Chorotype. - PAL.

MACROHABiTAT. - Alpine prairies.

NOTES. - Old record in need of validation.

\section{${ }^{*}$ Haplodrassus dalmatensis (L. Koch, 1866)}

NeW DATA. - Aymavilles: Pont d'Ael, 800 m, dry meadow, 3-24.V.2012, 1 0", leg. Negro.

Chorotype. - PAL.

MACROHABitAT. - Shrublands.

Notes. - New record for Aosta Valley.
•Haplodrassus kulczynskii Lohmander, 1942

Literature. - Di Caporiacco 1928; [Rossi \& Bosio 2012].

Chorotype. - PAL.

MacrohabitaT. - Rocky lands.

NOTES. - Old record in need of validation.

\section{Haplodrassus signifer (C. L. Koch, 1839)}

NEW DATA. - Arvier: Lago di Lolair, $1175 \mathrm{~m}$, broadleaved forest, 3-24.V.2012, 1 o", leg. Negro; same locality, 24.V-13.VI.2012, 3 O $^{7}$ 1 \%, leg. Negro; same locality, 13.VI-4.VII.2012, 1 ơ, leg. Negro; Morgex-La Salle: Natural Reserve of Marais, 896 m, riparian forest, 11-26.VI.2013, 3 o 3 9, leg. Negro; Torgnon: Lo Ditor, 1950 m, peat bog, 11-26.VI.2013, $60^{\circ} 2$ \%, leg. Negro; same locality, 26.VI-12.VII.2013, 1 ơ 1 \%, leg. Negro; same locality, 26.VII-14.VIII.2013, 3 ơ, leg. Negro; Sarre-Saint Pierre: Monte Torretta, 900 m, dry meadow, 24.V-13.VI.2012, 1 ơ, leg. Negro; Brissogne, Saint-Marcel, Quart, Nus: Natural Reserve of Les Iles, 530 m, dry meadow, 14.VI-5.VII.2011, 1 ơ 2 \% , leg. Paschetta \& Giuliano; same locality, 11-26.VI.2013, 1 오, leg. Negro; same locality, 26.VI-12.VII.2013, 1 \%, leg. Negro; Challand-Saint-Victor: Natural Reserve of Lago di Villa, 820 m, broadleaved forest, 12 26.VII.2013, 1 \%, leg Negro; Courmayeur: Val Ferret, 1630 m, shrubland, 26.VI-12.VII.2013, 1 \% , leg. Negro; Saint Christophe: Tsatelet, $750 \mathrm{~m}$, broadleaved forest, 3-24.V.2012, 1 o", leg. Negro; Aymavilles: Pont d'Ael, 800 m, dry meadow, 13.VI-4.VII.2012, 2 \%, leg. Negro; Pont-Saint-Martin: Natural Reserve of Stagno di Holay, $702 \mathrm{~m}$, broadleaved forest (Castanea sativa), 11-26.VI.2013, 1 \%, leg. Negro; Saint Denis: Brison-Cly, 1000 m, dry meadow, 13.VI-4. VII.2012, $30^{7}$, leg. Negro; same locality, broadleaved forest, 13.VI4.VII.2012, 1 ơ $^{7} 1$ \% , leg. Negro; same locality, 24.V-13.VI.2012, 1 ơ, leg. Negro; Ayas: Antagnod, 1970-2070 m, ski pistes, 2007, leg. Franco \& Negro.

Literature. - Calloni 1889; Pavesi 1904; di Caporiacco 1928; Isaia 2000, [2005]; De Angelis \& Fantoni 2008; Negro et al. 2009, 2010, 2013; [Rossi \& Bosio 2012].

Chorotype. - OLA.

MacrohabitAT. - Broadleaved forests, coniferous forests, riparian habitats, wetlands, shrublands, alpine prairies, rocky lands, ski pistes, alpine pastures.

Haplodrassus silvestris (Blackwall, 1833)

NEW DATA. - Challand-Saint-Victor: Natural Reserve of Lago di Villa, 820 m, meadow, 11-26.VI.2013, 1 o", leg. Negro; same locality, broadleaved forest, 11-26.VI.2013, $10^{\prime \prime} 1$ \% , leg. Negro; same locality, 26.VI-12.VII.2013, 1 \%, leg. Negro (MNHN); same locality, 26.VI-12.VII.2013, 3 o", leg. Negro; same locality, 26.VI-12. VII.2013, 1 ơ 19 , leg. Negro (MRSN-VDA); same locality, 12-26. VII.2013, $1 \sigma^{7} 1$ \% , leg. Negro; same locality, 28.VIII-16.IX.2013, 1 , leg. Negro; Pont-Saint-Martin: Natural Reserve of Stagno di Holay, $702 \mathrm{~m}$, broadleaved forest (Castanea sativa), 26.VI-12. VII.2013, $20^{7} 1$ \% , leg. Negro; Torgnon: Lo Ditor, 1950 m, peat bog, 12-26.VII.2013, 1 ơ, leg. Negro.

Literature. — De Angelis \& Fantoni 2008; [Rossi \& Bosio 2012].

Chorotype. - PAL.

MACROHABITAT. - Broadleaved forests, alpine grasslands, wetlands. 
${ }^{*}$ Haplodrassus soerenseni (Strand, 1900)

NEW DATA. - Ayas: Antagnod, 1970-2070 m, conifer wood, leg. Franco \& Negro.

Chorotype. - PAL.

MACROHABITAT. - Coniferous forests.

Notes. - New record for Aosta Valley.

Haplodrassus umbratilis (L. Koch, 1866)

Literature. - Di Caporiacco 1928; [Isaia 2005]; Negro et al. 2009; [Rossi \& Bosio 2012].

Chorotype. - CAE.

MacrohabitaT. - Coniferous forests.

Micaria aenea Thorell, 1871

NEW DATA. — Torgnon: Lo Ditor, 1950 m, peat bog, 11-26.VI.2013, 1 , leg. Negro (MRSN-VDA); same locality, 12-26.VII.2013, 1 , leg. Negro; same locality, 26.VI-12.VII.2013, 2 \%, leg. Negro; Ayas: Antagnod, 1970-2070 m, alpine pasture, 2007, leg. Franco \& Negro.

Literature. - Negro et al. 2009; [Rossi \& Bosio 2012].

Chorotype. - OLA.

MACROHABITAT. - Wetlands, coniferous forests, alpine pastures.

Micaria alpina L. Koch, 1872

Literature. - Pesarini 2000; [Isaia 2005]; Negro et al. 2009, 2010; [Rossi \& Bosio 2012].

Chorotype. - OLA.

MacrohabitaT. - Coniferous forests.

*Micaria coarctata (Lucas, 1846)

NEW DATA. — Ayas: Antagnod, 1970-2070 m, 2007, leg. Franco \& Negro.

CHOROTYPe. - CAM.

Macrohabitat. - Coniferous forests.

Notes. - New record for Aosta Valley.

Micaria formicaria (Sundevall, 1831)

Literature. - Pesarini 1997; [Isaia 2005]; De Angelis \& Fantoni 2008; [Rossi \& Bosio 2012].

Chorotype. - PAL.

MacrohabitaT. - Not specified.

Notes. - See notes about $A$. labyrinthica.
Micaria fulgens (Walckenaer, 1802)

NEW DATA. - Pont-Saint-Martin: Natural Reserve of Stagno di Holay, 775 m, wet meadow, 13.VI-4.VII.2011, 1 ㅇ, leg. Paschetta \& Giuliano (MRSN-VDA); Torgnon: Lo Ditor, 1950 m, peat bog, 12-26.VII.2013, 1 ㅇ, leg. Negro; Saint Denis: Brison-Cly, 1000 m, broadleaved forest, 24.V-13.VI.2012, 1 ㅇ, leg. Negro; Ayas: Antagnod, 1970-2070 m, alpine pasture, leg. Franco \& Negro.

LiterATURE. - Calloni 1889; [Isaia 2005]; De Angelis \& Fantoni 2008; Negro et al. 2009; [Rossi \& Bosio 2012].

CHOROTYPe. - SIE.

MACROHABITAT. - Wetlands, broadleaved forests, alpine pastures.

Micaria pulicaria (Sundevall, 1831)

NEW DATA. - Arvier: Lago di Lolair, 1175 m, wet meadow, 14.VI-5. VII.2011, $2 \sigma^{\prime \prime}$, leg. Paschetta \& Giuliano; same locality, 29.VII-18. VIII.2011, $10^{\prime \prime} 2 \%$, leg. Paschetta \& Giuliano; Pont-Saint-Martin: Natural Reserve of Stagno di Holay, 775 m, wet meadow, 4-28. VII.2011, 1 \&, leg. Paschetta \& Giuliano (MRSN-VDA); MorgexLa Salle: Natural Reserve of Marais, 896 m, grove of reeds, 11-26. VI.2013, 1 ㅇ, leg. Negro; same locality, 28.VIII-16.IX.2013, 2 ㅇ, leg. Negro; Torgnon: Lo Ditor, 1950 m, peat bog, 26.VI-12. VII.2013, 2 o", leg. Negro.

Literature. - Negro et al. 2009; [Rossi \& Bosio 2012].

Chorotype. - OLA.

MACROHABITAT. - Wetlands, alpine prairies.

\section{Micaria rossica Thorell, 1875}

Literature. - Calloni 1889; Pesarini 2000; [Isaia 2005]; [Rossi \& Bosio 2012].

Chorotype. - OLA.

MaCrohabitAT. - Not specified.

\section{${ }^{*}$ Micaria silesiaca L. Koch, 1875}

NEW DATA. - Torgnon: Lo Ditor, 1950 m, peat bog, 26.VII-14. VIII.2013, 1 \%, leg. Negro; Ayas: Antagnod, 1970-2070 m, ski pistes, 2007, leg. Franco \& Negro.

Chorotype. - PAL.

MACROHABITAT. - Ski pistes, wetlands.

Notes. - New record for Aosta Valley.

\section{*Micaria subopaca Westring, 1861}

NEW DATA. - Ayas: Antagnod, 1970-2070 m, 2007, leg. Franco \& Negro.

CHOrotype. - SIE.

Macrohabitat. - Coniferous forests.

Notes. - New record for Aosta Valley. 
Nomisia aussereri (L. Koch, 1872)

NEW DATA. - Brissogne, Saint-Marcel, Quart, Nus: Natural Reserve of Les Îles, $530 \mathrm{~m}$, dry meadow, 29.VII-18.VIII.2011, $1 \mathrm{O}^{\text {", }}$ leg. Paschetta \& Giuliano; same locality, 18.VIII-16.IX.2011, $20^{7} 2$, leg. Paschetta \& Giuliano; same locality, 26.VII-14.VIII.2013, 4 o", leg. Negro; same locality, 28.VIII-16.IX.2013, 1 ơ 1 \%, leg. Negro.

Literature. - Isaia 2005; [Rossi \& Bosio 2012].

Chorotype. - PAL.

MACROHABITAT. - Shrublands.

${ }^{*}$ Nomisia exornata (C. L. Koch, 1839)

NEW DATA. - Gressan: Natural Reserve of Côte de Gargantua, 700 m, dry meadow, 24.V-13.VI.2012, 1 o", leg. Negro; Pont-SaintMartin: Natural Reserve of Stagno di Holay, 616 m, alpine grassland, 11-26.VI.2013, 1 ơ, leg. Negro; Sarre-Saint Pierre: Monte Torretta, 900 m, alpine grassland, 3-24.V.2012, 2 o", leg. Negro; same locality, 4-25.VII.2012, 1 \%, leg. Negro (MRSN-VDA); same locality, 24.V-13.VI.2012, 1 o', leg. Negro.

Chorotype. - TUE.

MACROHABITAT. - Shrublands, alpine grasslands.

Notes. - New record for Aosta Valley.

\section{-Parasyrisca vinosa (Simon, 1878)}

LITERATURE. - Calloni 1889; [Isaia 2005].

Chorotype. - EUR.

MacrohabitAT. - Not specified.

Notes. - Old record in need of validation. The occurrence of the species in Aosta Valley is also considered doubtful by Rossi \& Bosio (2012).

\section{*Phaeocedus braccatus (L. Koch, 1866)}

NeW DATA. - Aymavilles: Pont d'Ael, $800 \mathrm{~m}$, dry meadow, 25.VII20.VIII.2012, 1 \%, leg. Negro (MRSN-VDA).

Chorotype. - PAL.

Macrohabitat. - Shrublands.

Notes. - New record for Aosta Valley.

-Scotophaeus quadripunctatus (Linnaeus, 1758)

LITERATURE. — Calloni 1889; [Isaia 2005]

Chorotype. - EUR.

MACROHABITAT. - Not specified.

Notes. - Old record in need of validation. The occurrence of this species in Aosta Valley is also considered doubtful by Rossi \& Bosio (2012).

\section{-Scotophaeus scutulatus (L. Koch, 1866)}

Literature. - Pavesi 1904; [Isaia 2005]; [Rossi \& Bosio 2012].

CHOrotype. - SIE.

MacrohabitAT. - Not specified.

NOTES. - Old record in need of validation.

\section{Trachyzelotes pedestris (C. L. Koch, 1837)}

NEW DATA. - Saint Denis: Brison-Cly, $1000 \mathrm{~m}$, broadleaved forest, 25.VII-20.VIII.2012, 1 \% , leg. Negro; Challand-Saint-Victor: Natural Reserve of Lago di Villa, 820 m, meadow, 11-26.VI.2013, 15 ơ $^{7} 2$ \%, leg. Negro;

SAME LOCALiTy. - 26.VI-12.VII.2013, $10^{\top 7} 2$ \% leg. Negro; same locality, broadleaved forest, 11-26.VI.2013, $40^{7} 1$ \% , leg. Negro; same locality, 26.VI-12.VII.2013, $10^{7} 1$ \%, leg. Negro (MRSNVDA); same locality, 26.VI-12.VII.2013, 2 o", leg. Negro; same locality, 12-26.VII.2013, 1 ơ 1 \%, leg. Negro; Pont-Saint-Martin: Natural Reserve of Stagno di Holay, 775 m, wet meadow, 28.VII-17. VIII.2011, 1 ㅇ, leg. Paschetta \& Giuliano (MNHN); same locality, broadleaved forest (Castanea sativa), 11-26.VI.2013, $60^{7}$, leg. Negro; same locality, 12-26.VII.2013, 1 \%, leg. Negro; same locality, alpine grassland, 11-26.VI.2013, $20^{\circ}$, leg. Negro; same locality, 26.VII-14.VIII.2013, $10^{\prime \prime}$, leg. Negro; Morgex-La Salle: Natural Reserve of Marais, 896 m, grove of reeds, 28.VIII-16.IX.2013, 1 오, leg. Negro; Saint Christophe: Tsatelet, $750 \mathrm{~m}$, broadleaved forest, 24.V-13.VI.2012, 1 o', leg. Negro; same locality, 4-25.VII.2012, $1 \%$, leg. Negro.

Literature. - Rossi \& Arnò 1995; Isaia 2005; De Angelis \& Fantoni 2008; [Rossi \& Bosio 2012].

Chorotype. - EUR.

MACROHABITAT. - Broadleaved forests, alpine grasslands, wetlands, shrublands.

\section{*Zelotes aeneus (Simon, 1878)}

NEW DATA. - Saint Denis: Brison-Cly, 1000 m, broadleaved forest, 25.VII-20.VIII.2012, 1 \%, leg. Negro.

Chorotype. - EUR.

MACROHABITAT. - Broadleaved forests.

Notes. - New record for Aosta Valley.

Zelotes apricorum (L. Koch, 1876)

NEW DATA. - Brissogne, Saint-Marcel, Quart, Nus: Natural Reserve of Les Îles, $530 \mathrm{~m}$, riparian vegetation, 14.VI-5.VII.2011, 1 일 leg. Paschetta \& Giuliano; same locality, 5-29.VII.2011, 1 ㅇ, leg. Paschetta \& Giuliano; same locality, 29.VII-18.VIII.2011, 7 ơ 9 ㅇ, leg. Paschetta \& Giuliano; same locality, 18.VIII-16. IX.2011, 3 ơ 4\%, leg. Paschetta \& Giuliano; same locality, dry meadow, 5-29.VII.2011, 1 \%, leg. Paschetta \& Giuliano; same locality, 18.VIII-16.IX.2011, 1 o' 1 \%, leg. Paschetta \& Giuliano; same locality, riparian forest, 11-26.VI.2013, 1 o', leg. Negro; same locality, 26.VI-12.VII.2013, 1 \%, leg. Negro; same locality, 12-26.VII.2013, 1 \%, leg. Negro; same locality, 26.VII-14. VIII.2013, 1 \%, leg. Negro; same locality, 14-28.VIII.2013, $10^{\text {" }}$ 
2 ㅇ, leg. Negro; same locality, 28.VIII-16.IX.2013, 1 ơ, leg. Negro; Courmayeur: Val Ferret, 1630 m, shrubs, 26.VI-12.VII.2013, 1 \% , leg. Negro; Saint Christophe: Tsatelet, $750 \mathrm{~m}$, broadleaved forest, 3-24.V.2012, 1 o' 1 \% , leg. Negro; same locality, 2 o", leg. Negro; same locality, 13.VI-4.VII.2012, 2 ơ 2 \%, leg. Negro; Aymavilles: Pont d'Ael, $800 \mathrm{~m}$, broadleaved forest, 3-24.V.2012, 2 \%, leg. Negro; same locality, dry meadow, 3-24.V.2012, 1 \% , leg. Negro; Challand-Saint-Victor: Natural Reserve of Lago di Villa, $820 \mathrm{~m}$, wet meadow, 13.VI-4.VII.2011, 1 \% , leg. Paschetta \& Giuliano (MRSN-VDA); same locality, 18.VIII-15.IX.2011, 2 o", leg. Paschetta \& Giuliano; Sarre-Saint Pierre: Monte Torretta, $900 \mathrm{~m}$, broadleaved forest, 13.VI-4.VII.2012, 1 \% , leg. Negro; Saint Denis: Brison-Cly, $1000 \mathrm{~m}$, broadleaved forest xerico, $24 . \mathrm{V}-13$. VI.2012, 1 ㅇ, leg. Negro; Morgex-La Salle: Natural Reserve of Marais, 900 m, riparian forest, 5-29.VII.2011, 1 \%, leg. Paschetta \& Giuliano; Gressan: Natural Reserve of Côte de Gargantua, $700 \mathrm{~m}$, broadleaved forest, 3-24.V.2012, 1 \% , leg. Negro; Arvier: Lago di Lolair, 1175 m, broadleaved forest, 3-24.V.2012, $10^{\circ}$, leg. Negro; same locality, 24.V-13.VI.2012, 1 o", leg. Negro; same locality, 13.VI-4.VII.2012, 1 \%, leg. Negro; same locality, 24.V-13.VI.2012, 1 ơ, leg. Negro.

Literature. - Isaia 2005; De Angelis \& Fantoni 2008; Negro et al. 2009; [Rossi \& Bosio 2012].

CHOROTYPe. - SIE.

MACROHABITAT. - Riparian habitats, shrublands, broadleaved forests, wetlands, coniferous forests.

Notes. - According to Grimm (1985), two morphotypes of $Z$. apricorum can be distinguished. As expected, the examined material in this work corresponds to the Southern morphotype.

\section{Zelotes clivicola (L. Koch, 1870)}

NEW DATA. - Pont-Saint-Martin: Natural Reserve of Stagno di Holay, 775 m, wet meadow, 13.VI-4.VII.2011, 1 \&, leg. Paschetta \& Giuliano.

Literature. - Calloni 1889; [Isaia 2005]; Negro et al. 2010; [Rossi \& Bosio 2012].

CHOROTYPE. - SIE.

MACROHABITAT. - Wetlands.

\section{Zelotes devotus Grimm, 1982}

Literature. - Gobbi et al. 2010; Negro et al. 2010; [Rossi \& Bosio 2012].

Chorotype. - ALP.

Macrohabitat. - Coniferous forests.

\section{Zelotes electus (C. L. Koch, 1839)}

NEW DATA. - Pont-Saint-Martin: Natural Reserve of Stagno di Holay, 775 m, wet meadow, 4-28.VII.2011, 1 \%, leg. Paschetta \& Giuliano (MNHN); Challand-Saint-Victor: Natural Reserve of Lago di Villa, 820 m, wet meadow, 13.VI-4.VII.2011, 3 ơ, leg. Paschetta \& Giuliano; Brissogne, Saint-Marcel, Quart, Nus: Natural Reserve of Les Îles, 530 m, dry meadow, 14.VI-5.VII.2011, $20^{7}$ 2 \%, leg. Paschetta \& Giuliano; same locality, 18.VIII-16.IX.2011,
1 , leg. Paschetta \& Giuliano; same locality, 14.VI-5.VII.2011, 10 , leg. Paschetta \& Giuliano; same locality, riparian vegetation, 14.VI-5.VII.2011, 1 \%, leg. Paschetta \& Giuliano.

Literature. - Rossi \& Arnò 1995; Isaia 2005; De Angelis \& Fantoni 2008; Negro et al. 2009; [Rossi \& Bosio 2012].

CHOROTYPe. - SIE.

MACROHABITAT. - Wetlands, shrublands, riparian habitats, alpine prairies.

\section{*Zelotes gallicus Simon, 1914}

NEW DATA. - Pont-Saint-Martin: Natural Reserve of Stagno di Holay, 616 m, alpine grassland, 26.VII-14.VIII.2013, 1 o", leg. Negro; same locality, 14-28.VIII.2013, 5 ơ, leg. Negro; same locality, 28.VIII-16.IX.2013, 5 ơ 2 \% , leg. Negro; Arvier: Lago di Lolair, $1175 \mathrm{~m}$, broadleaved forest, 25.VII-20.VIII.2012, $1 \mathrm{o}^{\circ}$, leg. Negro (MRSN-VDA); same locality, 25.VII-20.VIII.2012, $40^{7}$, leg. Negro; Courmayeur: Val Ferret, 1630 m, shrubs, 26.VII-14.VIII.2013, $20^{7} 1$ \% , leg. Negro.

Chorotype. - EUR.

MACROHABITAT. - Alpine grasslands, broadleaved forests, shrublands.

Notes. - New record for Aosta Valley.

\section{Zelotes latreillei (Simon, 1878)}

NEW DATA. - Arvier: Lago di Lolair, 1175 m, wet meadow, 14.VI-5. VII.2011, 1 \%, leg. Paschetta \& Giuliano; same locality, 18.VIII-16. IX.2011, 1 \% leg. Paschetta \& Giuliano; same locality, 29.VII-18. VIII.2011, 1 \%, leg. Paschetta \& Giu-liano; Courmayeur: Val Ferret, 1630 m, shrubs, 14-28.VIII.2013, 1 o", leg. Negro; same locality, 28.VIII-16.IX.2013, $10^{7} 2$ \% , leg. Negro; Verrayes: stagno di Loson, $1520 \mathrm{~m}$, grove of reeds/peat bog, 13.VI-4.VII.2011, $10^{7}$, leg. Paschetta \& Giuliano (MRSN-VDA); Ayas: Antagnod, 19702070 m, ski pistes, 2007, leg. Franco \& Negro.

Literature. - Rossi \& Arnò 1995; [Isaia 2005]; Negro et al. 2009; [Rossi \& Bosio 2012].

CHORotype. - SIE.

MACROHABITAT. - Wetlands, ski pistes, shrublands, alpine prairies.

\section{Zelotes longipes (L. Koch, 1866)}

NeW DATA. - Brissogne, Saint-Marcel, Quart, Nus: Natural Reserve of Les Îles, 530 m, dry meadow, 14.VI-5.VII.2011, 2 \%, leg. Paschetta \& Giuliano; same locality, 5-29.VII.2011, 1 o $^{\text {" } 1}$ \%, leg. Paschetta \& Giuliano; same locality, 29.VII-18.VIII.2011, 7 o", leg. Paschetta \& Giuliano; same locality, 18.VIII-16.IX.2011, 9 o + , leg. Paschetta \& Giuliano; Sarre-Saint Pierre: Monte Torretta, 900 m, dry meadow, 4-25.VII.2012, 1 \%, leg. Negro (MRSNVDA); Aymavilles: Pont d'Ael, $800 \mathrm{~m}$, dry meadow, 4-25.VII.2012, 1 ơ, leg. Negro (MRSN-VDA); same locality, 4-25.VII.2012, 1 \%, leg. Negro (MNHN).

Literature. - Rossi \& Arnò 1995; [Isaia 2005]; De Angelis \& Fantoni 2008; [Rossi \& Bosio 2012]. 
Chorotype. - PAL.

Macrohabitat. - Shrublands.

•Zelotes mundus (Kulczyński, 1897)

Literature. - Rossi \& Bosio 2012.

Chorotype. - PAL.

MACROHABITAT. - Riparian habitats.

Notes. - Doubtful. According Rossi \& Bosio (2012) this species is new for Italy. Given the method used for identification (see notes about Tegenaria domestica) and the lack of corresponding reference material, the record is also listed in the species of doubtful occurrence in Italy (Pantini \& Isaia 2015).

\section{*Zelotes paroculus Simon, 1914}

NEW DATA. - Pont-Saint-Martin: Natural Reserve of Stagno di Holay, 616 m, alpine grassland, 11-26.VI.2013, 1 o", leg. Negro (MRSN-VDA).

Chorotype. - WEU.

MacrohabitaT. - Alpine grasslands.

Notes. - New record for Aosta Valley.

\section{Zelotes petrensis (C. L. Koch, 1839)}

NEW DATA. - Saint Denis: Brison-Cly, $1000 \mathrm{~m}$, dry meadow, 24.V-13.VI.2012, 1 \%, leg. Negro; same locality, 13.VI-4. VII.2012, 1 \% , leg. Negro; same locality, 25.VII-20.VIII.2012, 2 \&, leg. Negro; same locality, broadleaved forest, 24.V-13. VI.2012, 1 o' 2 \%, leg. Negro; same locality, 13.VI-4.VII.2012, $10^{x}$, leg. Negro; same locality, 4-25.VII.2012, 1 ơ, leg. Negro; Pont-Saint-Martin: Natural Reserve of Stagno di Holay, 616 m, alpine grassland, 28.VIII-16.IX.2013, 5 o", leg. Negro; Torgnon: Lo Ditor, 1950 m, peat bog, 26.VI-12.VII.2013, 1 ơ", leg. Negro; Brissogne, Saint-Marcel, Quart, Nus: Natural Reserve of Les Iles, 526 m, dry meadow, 26.VI-12.VII.2013, 1 \& , leg. Negro; Aymavilles: Pont d'Ael, 800 m, dry meadow, 25.VII-20.VIII.2012, $10^{\pi} 1$ ㅇ, leg. Negro.

Literature. — De Angelis \& Fantoni 2008; [Rossi \& Bosio 2012].

CHOROTYPe. - SIE.

MACROHABITAT. - Shrublands, alpine grasslands, wetlands, broadleaved forests.

\section{Zelotes subterraneus (C. L. Koch, 1833)}

NEW DATA. - Courmayeur: Val Ferret, 1700 m, peat bog, 11.VII.2012, 1 \%, leg. Paschetta, Giuliano \& Mammola; Courmayeur: Val Ferret, $1630 \mathrm{~m}$, shrubs, 26.VI-12.VII.2013, $1 \mathrm{o}^{\text {", }}$ leg. Negro; Torgnon: Lo Ditor, $1950 \mathrm{~m}$, peat bog, 26.VII-14. VIII.2013, 1 ơ, leg. Negro; same locality, 12-26.VII.2013, 1 ㅇ, leg. Negro; Ayas: Antagnod, 1970-2070 m, alpine pasture, 2007, leg. Franco \& Negro; Ayas: Champoluc, 1650-1850 m, ski pistes, 2007, leg. Franco \& Negro.
Literature. - Grimm 1985; [Isaia 2005]; De Angelis \& Fantoni 2008; Negro et al. 2009; [Rossi \& Bosio 2012].

Chorotype. - PAL.

MACROHABITAT. - Alpine pastures, ski pistes, wetlands, shrublands.

\section{Zelotes talpinus (L. Koch, 1872)}

NEW DATA. - Torgnon: Lo Ditor, 1950 m, peat bog, 26.VII-14. VIII.2013, 7 ơ', leg. Negro; Arvier: Lago di Lolair, 1175 m, broadleaved forest, 13.VI-4.VII.2012, 1 \%, leg. Negro (MRSN-VDA); Ayas: Antagnod, 1970-2070 m, ski pistes, 2007, leg. Franco \& Negro; same locality, alpine pasture, 2007, leg. Franco \& Negro.

Literature. - Calloni 1889; di Caporiacco 1928; [Isaia 2005]; De Angelis \& Fantoni 2008; Negro et al. 2009, 2010; [Rossi \& Bosio 2012].

Chorotype. - SEU.

MACROHABITAT. - Broadleaved forests, alpine pastures, ski pistes, wetlands.

Family HAHNIIDAE Bertkau, 1878

Antistea elegans (Blackwall, 1841)

NEW DATA. - Verrayes: stagno di Loson, 1520 m, grove of reeds/ peat bog, 13.VI-4.VII.2011, 1 9 , leg. Paschetta \& Giuliano (MRSNVDA); same locality, 28.VII-17.VIII.2011, 1 ơ 2 \% , leg. Paschetta $\&$ Giuliano; Arvier: Lago di Lolair, 1175 m, grove of reeds, 5-29. VII.2011, 1 \%, leg. Paschetta \& Giuliano; same locality, wet meadow, 18.VIII-16.IX.2011, 1 O $^{7}$, leg. Paschetta \& Giuliano.

LiTERATURE. - Isaia 2005; De Angelis \& Fantoni 2008; [Rossi \& Bosio 2012].

Chorotype. - PAL.

MACROHABITAT. - Wetlands.

\section{Cryphoeca silvicola (C. L. Koch, 1834)}

NeW DATA. - Torgnon: Lo Ditor, 1950 m, peat bog, 26.VI-12. VII.2013, 1 ơ, leg. Negro; Courmayeur: Val Ferret, 1630 m, shrubs, 28.VIII-16.IX.2013, 1 o", leg. Negro; Ayas: Champoluc, 16501850 m, coniferous forests, 2007, leg. Franco \& Negro.

Literature. - Negro et al. 2009; [Rossi \& Bosio 2012].

Chorotype. - PAL.

MaCrohabitaT. - Coniferous forests, wetlands, shrublands.

\section{Habnia nava (Blackwall, 1841)}

Literature. - Isaia 2005; Negro et al. 2009; [Rossi \& Bosio 2012].

Chorotype. - SIE.

Macrohabitat. - Coniferous forests. 
Family LINYPHIIDAE Blackwall, 1859

Agyneta alpica Tanasevitch, 2000

Literature. - Negro et al. 2010, 2013; [Rossi \& Bosio 2012].

Chorotype. - ALP.

Macrohabitat. - Alpine prairies.

${ }^{*}$ Agyneta cauta (O. P.-Cambridge, 1902)

NEW DATA. - Courmayeur: Val Ferret, $1630 \mathrm{~m}$, shrubs, 26.VI-12. VII.2013, 1 ơ $^{7}$ leg. Negro (MRSN-VDA); same locality, 26.VI-12. VII.2013, 4 ơ 3 \% , leg. Negro; Torgnon: Lo Ditor, $1950 \mathrm{~m}$, peat bog, 12-26.VII.2013, 19 , leg. Negro.

Chorotype. - ASE.

MACROHABiTAT. - Shrublands, wetlands.

Notes. - New record for Aosta Valley.

Agyneta conigera (O. P.-Cambridge, 1863)

Literature. - Negro et al. 2009; [Rossi \& Bosio 2012].

CHORotype. - ASE.

Macrohabitat. - Coniferous forests.

-Agyneta gulosa (L. Koch, 1869)

Literature. - Calloni 1889; [Isaia 2005].

CHOROTYPE. - PAL.

Macrohabitat. - Not specified.

NOTES. - Old record in need of validation. The occurrence of the species in Aosta Valley is considered doubtful by Rossi \& Bosio (2012).

\section{-Agyneta nigripes (Simon, 1884)}

Literature. — Calloni 1889; [Pavesi 1904]; [Isaia 2005].

Chorotype. - PAL.

Macrohabitat. - Not specified.

NOTES. - Old record in need of verification. The occurrence of the species in Aosta Valley is also considered doubtful by Rossi \& Bosio (2012).

Agyneta orites (Thorell, 1875)

NEW DATA. — Ayas: Antagnod, 1970-2070 m, alpine pasture, 2007, $30^{*} 3$ \%, leg. Franco \& Negro.

Literature. - Negro et al. 2009.
Chorotype. - SEU.

MACROHABITAT. - Alpine pastures.

Agyneta rurestris (C. L. Koch, 1836)

NEW DATA. - Pont-Saint-Martin: Natural Reserve of Stagno di Holay, 775 m, wet meadow, 13.VI-4.VII.2011, 1 \% , leg. Paschetta \& Giuliano; Morgex-La Salle: Natural Reserve of Marais, 900 m, meadow/grove of reeds, 9.VII.2012, 1 o", leg. Paschetta.

Literature. — Isaia 2000, [2005]; [Rossi \& Bosio 2012].

CHOrotype. - PAL.

MacrohabitaT. - Wetlands, alpine prairies.

-Agyneta saxatilis (Blackwall, 1844)

LiterATURe. - Rossi \& Bosio 2012.

СHorotype. - SIE.

Macrohabitat. - Coniferous forests.

Notes. - See Tegenaria domestica.

Anguliphantes monticola (Kulczyński, 1881)

Literature. - Calloni 1889; [Pavesi 1904]; [Isaia 2005]; Negro et al. 2010.

CHOrotype. - EUR.

Macrohabitat. - Not specified.

NoTES. — Records by Calloni (1889) possibly refer to Simon (1881), reporting material collected in Valais (Switzerland).

Araeoncus anguineus (L. Koch, 1869)

Literature. - Negro et al. 2010; [Rossi \& Bosio 2012].

CHOrotype. - SEU.

MACrOHABITAT. - Alpine prairies.

\section{Asthenargus paganus (Simon, 1884)}

Literature. — Negro et al. 2009; [Rossi \& Bosio 2012].

Chorotype. - SIE.

MACROHABITAT. - Alpine prairies.

*Bathyphantes gracilis (Blackwall, 1841)

NeW DaTA. - La Thuile: Comba Thuilette, $2000 \mathrm{~m}$, peat bog, 17.VIII.2011, 2 \%, leg. Paschetta \& Giuliano; Verrayes: stagno di Loson, 1520 m, peat bog, 4-28.VII.2011, 1 \% , leg. Paschetta \& 
Giuliano; same locality, 28.VII-17.VIII.2011, 2 \%, leg. Paschetta \& Giuliano.

Chorotype. - OLA.

MACROHABITAT. - Wetlands.

Notes. - New record for Aosta Valley. Rossi \& Arnò (1995) reported Bathyphantes prope gracilis for the Natural Reserve of Les Iles. Accordingly, Rossi \& Bosio (2012) reported the same citation for this species as doubtful.

*Bathyphantes similis Kulczyński, 1894

NEW DATA. - Brissogne, Saint-Marcel, Quart, Nus: Natural Reserve of Les Îles, $530 \mathrm{~m}$, riparian vegetation, 5-29.VII.2011, 1 \%, leg. Paschetta \& Giuliano; same locality, 18.VIII-16.IX.2011, 1 \%, leg. Paschetta \& Giuliano.

Chorotype. - EUR.

MACROHABITAT. - Riparian habitats.

Notes. - New record for Aosta Valley.

*Bolyphantes luteolus (Blackwall, 1833)

New DATA. - La Thuile: Comba Thuilette, 2000 m, peat bog, 13.IX.2012, 1 \%, leg. Paschetta, Mammola \& Marguerettaz.

Chorotype. - PAL.

MACROHABITAT. - Wetlands.

Notes. - New record for Aosta Valley.

Caracladus avicula (L. Koch, 1869)

NEW DATA. - Ayas: Antagnod, 1970-2070 m, alpine pasture, 2007, leg. Franco \& Negro; Ayas: Champoluc, 1970-2070 m, ski pistes, 2007, leg. Franco \& Negro.

Literature. - Pesarini 1996; [Isaia 2005]; [Rossi \& Bosio 2012].

Chorotype. - SEU.

Macrohabitat. - Alpine pastures, ski pistes.

Centromerita bicolor (Blackwall, 1833)

Literature. - Negro et al. 2009; [Rossi \& Bosio 2012].

Chorotype. - OLA.

MacrohabitaT. - Coniferous forests.

Centromerus brevipalpus (Menge, 1866)

Literature. — Negro et al. 2009; [Rossi \& Bosio 2012].
Chorotype. - ASE.

Macrohabitat. - Coniferous forests.

Centromerus subalpinus Lessert, 1907

LiteratURe. — Negro et al. 2009; [Rossi \& Bosio 2012].

Chorotype. - SEU.

MaCrohabitat. - Alpine prairies.

Centromerus sylvaticus (Blackwall, 1841)

NEW DATA. - Brissogne, Saint-Marcel, Quart, Nus: Natural Reserve of Les Îles, $530 \mathrm{~m}$, riparian forest, 14.VI-5.VII.2011, 1 \% , leg. Paschetta \& Giuliano.

Literature. - Isaia 2005; [Rossi \& Bosio 2012].

Chorotype. - OLA.

MACROHABITAT. - Riparian habitats.

${ }^{*}$ Ceratinella brevipes (Westring, 1851)

NEW DATA. - Verrayes: stagno di Loson, 1520 m, grove of reeds/ peat bog, 13.VI-4.VII.2011, $10^{7} 6$ 우, leg. Paschetta \& Giuliano; same locality, 13.VI-4.VII.2011, 1 o", leg. Paschetta \& Giuliano (MRSN-VDA); same locality, 4-28.VII.2011, 1 \%, leg. Paschetta \& Giuliano; same locality, 28.VII-17.VIII.2011, 1 \%, leg. Paschetta \& Giuliano.

CHorotype. - SEU.

Macrohabitat. - Wetlands.

Notes. - New record for Aosta Valley.

\section{Ceratinella brevis (Wider, 1834)}

NeW DATA. - Brissogne, Saint-Marcel, Quart, Nus: Natural Reserve of Les Îles, 530 m, riparian forest, 5-29.VII.2011, 1 \%, leg. Paschetta \& Giuliano; same locality, 18.VIII-16.IX.2011, $10^{7}$, leg. Paschetta \& Giuliano; Ayas: Antagnod, 1970-2070 m, 2007, leg. Franco \& Negro.

LiTERATURE. — Pavesi 1904.

Chorotype. - PAL.

MACROHABITAT. - Riparian habitats.

Ceratinella scabrosa (O. P.-Cambridge, 1871)

Literature. - Negro et al. 2009; [Rossi \& Bosio 2012].

Chorotype. - PAL.

Macrohabitat. - Coniferous forests. 
Collinsia inerrans (O. P.-Cambridge, 1885)

NEW DATA. — Arvier: Lago di Lolair, 1175 m, wet meadow, 29.VII18.VIII.2011, $10^{7} 1$ \%, leg. Paschetta \& Giuliano; same locality, 14.VI-5.VII.2011, 1 ơ, leg. Paschetta \& Giuliano (MRSN-VDA).

LiterATURE. - Pantini \& Isaia 2008; Negro et al. 2009; [Rossi \& Bosio 2012].

Chorotype. - PAL.

MACROHABITAT. - Wetlands, alpine pastures.

Diplocentria bidentata (Emerton, 1882)

NEW DATA. - Ayas: Antagnod, 1970-2070 m, ski pistes, 2007, leg. Franco \& Negro.

LiteratURE. - Pantini \& Isaia 2008; Negro et al. 2009; [Rossi \& Bosio 2012].

Chorotype. - OLA.

Macrohabitat. - Coniferous forests, ski pistes.

Diplocephalus cristatus (Blackwall, 1833)

Literature. — Rossi \& Arnò 1995; [Isaia 2005]; [Rossi \& Bosio 2012].

Chorotype. - OLA.

MacrohabitaT. - Shrublands.

-Diplocephalus helleri (L. Koch, 1869)

Literature. — Calloni 1889; [Pavesi 1904]; [Isaia 2005].

Chorotype. - CEU.

MACROHABITAT. - Not specified.

Notes. - Old record in need of verification. The occurrence of this species in Aosta Valley is also considered doubtful by Rossi \& Bosio (2012).

\section{Diplocephalus latifrons (O. P.-Cambridge, 1863)}

NEW DATA. - Arvier: Lago di Lolair, 1175 m, wet meadow, 14.VI5.VII.2011, 1 o 1 ㅇ, leg. Paschetta \& Giuliano, Morgex-La Salle: Natural Reserve of Marais, 900 m, riparian forest, 14.VI-5.VII.2011, $20^{7} 1$ \% , leg. Paschetta \& Giuliano; same locality, 5-29.VII.2011, $10^{7}$, leg. Paschetta, Giuliano (MRSN-VDA); same locality, 29.VII-18. VIII.2011, 1 \&, leg. Paschetta, Giuliano; same locality, wet meadow, 5-29.VII.2011, 2 o", leg. Paschetta, Giuliano; Pont-Saint-Martin: Natural Reserve of Stagno di Holay, 679 m, broadleaved forest (Castanea sativa), 11-26.VI.2013, 1 o", leg. Negro (MNHN).

Literature. - Rossi \& Arnò 1995; Negro et al. 2009; [Rossi \& Bosio 2012].

CHOROTyPe. - EUR.

MACROHABITAT. - Wetlands, riparian habitats, broadleaved forests.
Diplostyla concolor (Wider, 1834)

NeW DATA. - Brissogne, Saint-Marcel, Quart, Nus: Natural Reserve of Les Îles, 530 m, dry meadow, 14.VI-5.VII.2011, 3 \% , leg. Paschetta \& Giuliano; same locality, 5-29.VII.2011, 1 \%, leg. Paschetta \& Giuliano; same locality, 18.VIII-16.IX.2011, $10^{7} 2$ \%, leg. Paschetta \& Giuliano; same locality, riparian vegetation, 14.VI-5.VII.2011, 2 ơ 5 \%, leg. Paschetta \& Giuliano; same locality, 5-29.VII.2011, 8 o7 7 \% , leg. Paschetta \& Giuliano; same locality, 29.VII-18.VIII.2011, 6 ơ 9 9 , leg. Paschetta \& Giuliano; same locality, 18.VIII-16.IX.2011, $60^{\prime \prime} 15$ \%, leg. Paschetta \& Giuliano; Challand-Saint-Victor: Natural Reserve of Lago di Villa, $820 \mathrm{~m}$, riparian forest, 13.VI-4.VII.2011, $4 \mathrm{o}^{7}$, leg. Paschetta \& Giuliano; same locality, 18.VIII-15.IX.2011, $10^{\prime \prime}$, leg. Paschetta \& Giuliano; same locality, wet meadow, 13.VI-4.VII.2011, $20^{\top 7} 2$ 우 leg. Paschetta \& Giuliano; same locality, broadleaved forest, 11-26. VI.2013, 3 \% , leg. Negro; same locality, 12-26.VII.2013, $10^{*}$, leg. Negro; same locality, 26.VII-14.VIII.2013, 9 ơ 6\%, leg. Negro; same locality, 14-28.VIII.2013, 1 \% , leg. Negro; Courmayeur: Val Ferret, 1779 m, shrubland, 28.VIII-16.IX.2013, 1 \&, leg. Negro; Arvier: Lago di Lolair, 1175 m, wet meadow, 14.VI-5.VII.2011, $30^{7}$, leg. Paschetta \& Giuliano; same locality, 5-29.VII.2011, $20^{\prime \prime}$ 1 \%, leg. Paschetta \& Giuliano; same locality, 18.VIII-16.IX.2011, 10 , leg. Paschetta \& Giuliano; Morgex-La Salle: Natural Reserve of Marais, $900 \mathrm{~m}$, riparian forest, 14.VI-5.VII.2011, $90^{7} 4 \mathrm{o}^{7}$, leg. Paschetta \& Giuliano; same locality, 5-29.VII.2011, $20^{*} 3$ \%, leg. Paschetta \& Giuliano; same locality, 29.VII-18.VIII.2011, $20^{\text {" }}$ $2 \%$, leg. Paschetta \& Giuliano; same locality, 18.VIII-16.IX.2011, 1 ơ $^{\prime} 2$ \% leg. Paschetta \& Giuliano; same locality, 5-29.VII.2011, 10 \% , leg. Paschetta \& Giuliano; same locality, 29.VII-18.VIII.2011, 4 ơ, leg. Paschetta \& Giuliano; Pont-Saint-Martin: Natural Reserve of Stagno di Holay, $679 \mathrm{~m}$, broadleaved forest (Castanea sativa), 11-26.VI.2013, 1 \%, leg. Negro; same locality, 12-26.VII.2013,

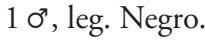

Literature. — Rossi \& Arnò 1995; [Isaia 2005]; Negro et al. 2009; [Rossi \& Bosio 2012].

Chorotype. - OLA.

MACROHABITAT. - Riparian habitats, broadleaved forests, wetlands, shrublands, coniferous forests.

\section{Drapetisca socialis (Sundevall, 1833)}

NEW DATA. - Morgex-La Salle: Natural Reserve of Marais, 900 m, riparian forest, 16.VIII.2011, 1 \%, leg. Paschetta \& Giuliano (MRSNVDA).

Literature. — De Angelis \& Fantoni 2008; [Rossi \& Bosio 2012].

Chorotype. - PAL.

MACROHABITAT. - Riparian habitats.

\section{${ }^{*}$ Entelecara acuminata (Wider, 1834)}

NEW DATA. - Morgex-La Salle: Natural Reserve of Marais, 900 m, riparian forest, 9.VII.2012, 1 ơ $^{7}$ \%, leg. Paschetta; same locality, meadow/grove of reeds, 9.VII.2012, 1 o , leg. Paschetta.

Chorotype. - OLA.

MACROHABITAT. - Riparian habitats, wetlands.

Notes. - New record for Aosta Valley. 


\section{*Erigone atra Blackwall, 1833}

NEW DATA. — La Thuile: Comba Thuilette, 2000 m, peat bog, 10.VII.2012, 1 \&, leg. Paschetta, Giuliano, Mammola, \& Marguerettaz.

Chorotype. - OLA.

MACROHABITAT. - Wetlands.

Notes. - New record for Aosta Valley.

\section{Erigone cristatopalpus Simon, 1884}

New DATA. - La Thuile: Comba Thuilette, 2000 m, peat bog, 10.VII.2012, 1 ơ 2 ㅇ, leg. Paschetta, Giuliano, Mammola \& Marguerettaz; Courmayeur: Val Ferret, 1700 m, peat bog, 11.VII.2012, 1 ㅇ, leg. Paschetta, Giuliano \& Mammola.

Literature. — Calloni 1889; [Pavesi 1904]; [Isaia 2005].

Chorotype. - CEU.

MacrohabitAT. - Wetlands.

Notes. - The occurrence of this species in Aosta Valley, here confirmed by original data, was considered doubtful by Rossi \& Bosio (2012).

\section{Erigone dentipalpis (Wider, 1834)}

NEW DATA. - Arvier: Lago di Lolair, 1175 m, wet meadow, 14.VI-5.VII.2011, 6 ơ 1 ㅇ, leg. Paschetta \& Giuliano; same locality, 29.VII-18.VIII.2011, 1 Ơ $^{7} 1$ ㅇ, leg. Paschetta \& Giuliano; Pont-Saint-Martin: Natural Reserve of Stagno di Holay, 775 m, wet meadow, 13.VI-4.VII.2011, 1 ơ, leg. Paschetta, Giuliano (MRSN-VDA).

LiterATURE. - Rossi \& Arnò 1995; [Isaia 2005]; Negro et al. 2009 , 2010, 2013; [Rossi \& Bosio 2012].

Chorotype. - OLA.

MACROHABITAT. - Broadleaved forests, shrublands, wetlands, ski pistes.

•Erigone remota L. Koch, 1869

LiteraTure. - Calloni 1889; Pavesi 1904; [Isaia 2005]; [Rossi \& Bosio 2012].

Chorotype. - SIE.

MACrOHABITAT. - Not specified.

Notes. - Old record in need of verification.

${ }^{*}$ Erigonella subelevata (L. Koch, 1869)

NeW DaTA. - Ayas: Antagnod, 1970-2070 m, ski pistes, 2007, leg. Franco \& Negro.

Chorotype. - SEU.
MaCrohabitat. - Ski pistes.

Notes. - New record for Aosta Valley.

*Floronia bucculenta (Clerck, 1757)

NEW DATA. - Pont-Saint-Martin: Natural Reserve of Stagno di Holay, 775 m, riparian vegetation, 13.VIII.2012, 1 , leg. Paschetta.

Chorotype. - PAL.

MACROHABITAT. - Riparian habitats.

Notes. - New record for Aosta Valley.

Frontinellina frutetorum (C. L. Koch, 1834)

LITERATURE. - Isaia 2005; [Rossi \& Bosio 2012].

Chorotype. - SIE.

Macrohabitat. - Not specified.

\section{Gnathonarium dentatum (Wider, 1834)}

NEW DATA. - Verrayes: stagno di Loson, 1520 m, grove of reeds/ peat bog, 4-28.VII.2011, 1 ơ 2 ㅇ, leg. Paschetta \& Giuliano; same locality, 18.VIII-15.IX.2011, 2 \%, leg. Paschetta \& Giuliano; same locality, 13.VI-4.VII.2011, 2 \%, leg. Paschetta \& Giuliano; PontSaint-Martin: Natural Reserve of Stagno di Holay, 775 m, riparian vegetation, 5.IV.2012, $10^{7}$, leg. Paschetta \& Isaia.

Arvier: Lago di Lolair, 1175 m, wet meadow, 14.VI-5.VII.2011, 1 \&, leg. Paschetta \& Giuliano; same locality, 18.VIII-16.IX.2011, 1 \%, leg. Paschetta \& Giuliano; same locality, 29.VII-18.VIII.2011, 1 ㅇ, leg. Paschetta \& Giuliano.

Literature. — Rossi \& Arnò 1995; [Isaia 2005]; [Rossi \& Bosio 2012].

CHOROTYPe. - PAL.

MACROHABITAT. - Wetlands, riparian habitats, shrublands.

Gonatium rubens (Blackwall, 1833)

Literature. - Negro et al. 2009; [Rossi \& Bosio 2012].

Chorotype. - ASE.

MaCrohabitaT. - Coniferous forests.

Gongylidiellum latebricola (O. P.-Cambridge, 1871)

LiterATURE. — Isaia 2000, [2005]; [Rossi \& Bosio 2012].

CHOROTYPe. - PAL.

MaCrohabitaT. - Rocky lands. 
${ }^{*}$ Hilaira excisa (O. P.-Cambridge, 1871)

NEW DATA. — Courmayeur: Val Ferret, 1700 m, peat bog, 11.VII.2012, 1 ㅇ, leg. Paschetta, Giuliano \& Mammola; same locality, 1630 m, shrubs, 26.VII-14.VIII.2013, 2 o", leg. Negro; same locality, 14-28. VIII.2013, 3 o", leg. Negro; La Thuile: Comba Thuilette, 2000 m, peat bog, 13.IX.2012, 1 \%, leg. Paschetta, Mammola \& Marguerettaz; Morgex-La Salle: Natural Reserve of Marais, 896 m, riparian forest, 11-26.VI.2013, 1 ơ, leg. Negro.

Chorotype. - EUR.

MACROHABitAT. - Shrublands, wetlands, riparian habitats.

Notes. - New record for Aosta Valley.

\section{Incestophantes frigidus (Simon, 1884)}

Literature. - Calloni 1889; Pavesi 1904; di Caporiacco 1928; [Isaia 2005]; Negro et al. 2009; [Rossi \& Bosio 2012].

Chorotype. - CEU.

Macrohabitat. - Alpine prairies.

${ }^{*}$ Centromerus pabulator (O. P.-Cambridge, 1875)

NEW DATA. - Ayas: Antagnod, 1970-2070 m, coniferous forests, 2007, 1 ㅇ, leg. Franco \& Negro.

Chorotype. - SIE.

MacrohabitaT. - Coniferous forests.

Notes. - New record for Aosta Valley.

\section{*Lepthyphantes nodifer Simon, 1884}

NEW DATA. - Torgnon: Lo Ditor, 1950 m, peat bog, 12-26.VII.2013, 1 \%, leg. Negro; Ayas: Champoluc, 1650-1850 m, coniferous forests, 2007, leg. Franco \& Negro.

Chorotype. - CEU.

MacrohabitaT. - Wetlands, coniferous forests.

Notes. - New record for Aosta Valley.

\section{${ }^{*}$ Lessertinella kulczynskii (Lessert, 1910)}

NEW DATA. — Courmayeur: Val Ferret, 1630 m, shrubs, 26.VII-14. VIII.2013, 1 on $^{n}$ leg. Negro,

Chorotype. - SEU.

Macrohabitat. - Shrublands.

Notes. - New record for Aosta Valley.
*Linyphia hortensis Sundevall, 1830

NEW DATA. - La Thuile: Comba Thuilette, 2000 m, riparian vegetation, 16.VIII.2011, 3 \%, leg. Paschetta \& Giuliano; Ayas: Champoluc, 1650-1850 m, ski pistes, 2007, leg. Franco \& Negro.

Chorotype. - PAL.

MACROHABITAT. - Riparian habitats, ski pistes.

Notes. - New record for Aosta Valley.

\section{Linyphia triangularis (Clerck, 1757)}

NEW DATA. - Challand-Saint-Victor: Natural Reserve of Lago di Villa, 820 m, riparian vegetation, 18.VIII.2011, 1 \%, leg. Paschetta \& Giuliano; same locality, grove of reeds, 14.IX.2012, 2 \%, leg. Paschetta \& Mammola; Morgex-La Salle: Natural Reserve of Marais, $900 \mathrm{~m}$, riparian vegetation, 16.VIII.2011, 2 \% , leg. Paschetta $\&$ Giuliano; Pont-Saint-Martin: Natural Reserve of Stagno di Holay, 775 m, riparian vegetation, 13.VIII.2012, 1 o", leg. Paschetta; same locality, 18.VIII.2011, 4 \%, leg. Paschetta \& Giuliano.

Literature. — Pavesi 1904; Rossi \& Arnò 1995; Isaia 2000, [2005]; De Angelis \& Fantoni 2008; [Rossi \& Bosio 2012].

Chorotype. - PAL.

MACROHABITAT. - Riparian habitats, wetlands, broadleaved forests, synanthropic habitats, shrublands, coniferous forests.

\section{*Macrargus rufus (Wider, 1834)}

NEW DATA. - Ayas: Champoluc, 1650-1850 m, coniferous forests, 2007, leg. Franco \& Negro.

CHOROTYPe. - SIE.

Macrohabitat. - Coniferous forests.

Notes. - New record for Aosta Valley.

\section{Mansuphantes pseudoarciger (Wunderlich, 1985)}

Literature. - Pantini \& Isaia 2008; Negro et al. 2009; [Rossi \& Bosio 2012].

Chorotype. - ALP.

Macrohabitat. - Coniferous forests.

\section{Maso sundevalli (Westring, 1851)}

NEW DATA. - Brissogne, Saint-Marcel, Quart, Nus: Natural Reserve of Les Îles, $526 \mathrm{~m}$, riparian forest, 26.VI-12.VII.2013, $1 \mathrm{o}^{7}$, leg. Negro.

Literature. - Rossi \& Arnò 1995; [Isaia 2005]; [Rossi \& Bosio 2012].

Chorotype. - OLA.

MacrohabitaT. - Shrublands, riparian habitats. 


\section{*Mecopisthes latinus Millidge, 1978}

NEW DATA. — Ayas: Antagnod, 1970-2070 m, ski pistes, 2007, 1 ơ 1 , leg. Franco \& Negro.

Chorotype. - APPC.

MacrohabitaT. - Ski pistes.

Notes. - New record for Aosta Valley.

•Mecynargus brocchus (L. Koch, 1872)

Literature. — Calloni 1889; [Pavesi 1904]; [Isaia 2005].

CHOROTYPe. - EUR.

MACROHABITAT. - Not specified.

Notes. - Old record in need of verification. The occurrence of the species in Aosta Valley is also considered doubtful by Rossi \& Bosio (2012).

•Mecynargus paetulus (O. P.-Cambridge, 1875)

Literature. — Calloni 1889; [Pavesi 1904]; [Isaia 2005].

Chorotype. - OLA.

Macrohabitat. - Not specified.

NOTES. - Old record in need of verification. The occurrence of the species in Aosta Valley is also considered doubtful by Rossi \&Bosio (2012).

*Mermessus trilobatus (Emerton, 1882)

NEW DATA. - Pont-Saint-Martin: Natural Reserve of Stagno di Holay, 775 m, wet meadow, 13.VI-4.VII.2011, 1 o", leg. Paschetta \& Giuliano.

Chorotype. - OLA.

MacrohabitAT. - Wetlands.

Notes. - New record for Aosta Valley.

Metopobactrus prominulus (O. P.-Cambridge, 1872)

Literature. — Negro et al. 2009; [Rossi \& Bosio 2012].

Chorotype. - OLA.

MACROHABITAT. - Coniferous forests.

*Micrargus alpinus Relys \& Weiss, 1997

NEW DATA. - Ayas: Antagnod, 1970-2070 m, coniferous forests, 1 , leg. Franco \& Negro.

Chorotype. - CEU.
Macrohabitat. - Coniferous forests.

Notes. - New record for Aosta Valley.

Micrargus apertus (O. P.-Cambridge, 1871)

NEW DATA. - Brissogne, Saint-Marcel, Quart, Nus: Natural Reserve of Les Îles, $526 \mathrm{~m}$, riparian forest, 26.VI-12.VII.2013, $1 \mathrm{o}^{\text {"⿻ }}$, leg. Negro; Pont-Saint-Martin: Natural Reserve of Stagno di Holay, $679 \mathrm{~m}$, broadleaved forest (Castanea sativa), 11-26.VI.2013, 1 o", leg. Negro (MRSN-VDA); Ayas: Antagnod, 1970-2070 m, coniferous forests, leg. Franco \& Negro.

Literature. - Negro et al. 2009; [Rossi \& Bosio 2012].

Chorotype. - PAL.

MACROHABITAT. - Riparian habitats, broadleaved forests, coniferous forests.

*Micrargus herbigradus (Blackwall, 1854)

NeW DATA. - La Thuile: Comba Thuilette, 2000 m, peat bog, 13.IX.2012, 1 ơ, leg. Paschetta, Mammola \& Marguerettaz.

Chorotype. - PAL.

MACROHABITAT. - Wetlands.

Notes. - New record for Aosta Valley.

${ }^{*}$ Micrargus subaequalis (Westring, 1851)

NEW DATA. - Morgex-La Salle: Natural Reserve of Marais, $900 \mathrm{~m}$, riparian forest, 14.VI-5.VII.2011, $80^{7} 2$ \% , leg. Paschetta \& Giuliano; same locality, wet meadow, 14.VI-5.VII.2011, 1 \%, leg. Paschetta \& Giuliano (MRSN-VDA); same locality, wet meadow, 5-29.VII.2011, $10^{7}$, leg. Paschetta \& Giuliano (MRSN-VDA); same locality, riparian forest, 5-29.VII.2011, $10^{7} 1$ ㅇ, leg. Paschetta \&Giuliano; same locality, 12-26.VII.2013, 1 ơ, leg. Negro; Brissogne, Saint-Marcel, Quart, Nus: Natural Reserve of Les Îles, $530 \mathrm{~m}$, riparian forest, 5-29.VII.2011, $1 \mathrm{o}^{\text {", }}$ leg. Paschetta \& Giuliano; same locality, 12-26.VII.2013, 3 o", leg. Negro; Challand-Saint-Victor: Natural Reserve of Lago di Villa, $820 \mathrm{~m}$, broadleaved forest, 12-26.VII.2013, 1 ơ, leg. Negro; same locality, meadow, 12-26.VII.2013, 1 ㅇ, leg. Negro; same locality, 26.VI-12.VII.2013, 1 ơ, leg. Negro; Courmayeur: Val Ferret, 1630 m, shrubs, 26.VII-14.VIII.2013, 1 \&, leg. Negro.

Chorotype. - ASE.

MACROHABITAT. - Riparian habitats, broadleaved forests, alpine grasslands, shrublands, wetlands.

Notes. - New record for Aosta Valley.

*Microlinyphia impigra (O. P. Cambridge, 1871)

NEW DATA. - Challand-Saint-Victor: Natural Reserve of Lago di Villa, 820 m, wet meadow, 18.VIII.2011, $10^{7}$, leg. Paschetta \& Giuliano (MRSN-VDA).

Chorotype. - OLA. 
Macrohabitat. - Wetlands.

Notes. - New record for Aosta Valley.

Microlinyphia pusilla (Sundevall, 1830)

NEW DATA. — Courmayeur: Val Ferret, 1700 m, peat bog, 11.VII.2012, 3 , leg. Paschetta, Giuliano \& Mammola.

LiterATURE. - Rossi \& Arnò 1995; [Isaia 2005]; [Rossi \& Bosio 2012].

Chorotype. - OLA.

MACROHABITAT. - Wetlands, shrublands.

Microneta viaria (Blackwall, 1841)

NEW DATA. - Arvier: Lago di Lolair, 1175 m, wet meadow, 18.VIII16.IX.2011, 1 \% , leg. Paschetta \& Giuliano; Challand-Saint-Victor: Natural Reserve of Lago di Villa, 820 m, broadleaved forest, 11-26. VI.2013, 1 \%, leg. Negro.

LiterATURE. - De Angelis \& Fantoni 2008; [Rossi \& Bosio 2012].

Chorotype. - OLA.

MACROHABITAT. - Wetlands, broadleaved forests.

Minyriolus pusillus (Wider, 1834)

Literature. - Negro et al. 2009; [Rossi \& Bosio 2012].

CHOROTYPe. - SIE.

MacrohabitaT. - Coniferous forests.

*Mughiphantes armatus (Kulczyński, 1905)

NEW DATA. - Ayas: Antagnod, 1970-2070 m, ski pistes, 2007, leg. Franco \& Negro.

Chorotype. - ALP.

Macrohabitat. - Ski pistes.

Notes. - New record for Aosta Valley.

*Mughiphantes cornutus (Schenkel, 1927)

NEW DATA. - Ayas: Antagnod, 1970-2070 m, ski pistes, 2007, leg. Franco \& Negro.

Chorotype. - ASE.

Macrohabitat. - Ski pistes.

Notes. - New record for Aosta Valley.
Mughiphantes handschini (Schenkel, 1919)

LiTERATURE. - Thaler 1982; [Isaia 2005]; Negro et al. 2010, 2013; [Rossi \& Bosio 2012].

Chorotype. - SEU.

MACROHABITAT. - Coniferous forests.

Mughiphantes pulcheroides (Wunderlich, 1985)

LiTERATURE. - Wunderlich 1985; [Isaia 2005]; [Rossi \& Bosio 2012].

Chorotype. - ALPW.

MACROHABITAT. - Rocky lands.

Neriene clathrata (Sundevall, 1830)

NEW DATA. - Challand-Saint-Victor: Natural Reserve of Lago di Villa, 820 m, wet meadow, 18.VIII.2011, 2 \%, leg. Paschetta \& Giuliano (MNHN); Brissogne, Saint-Marcel, Quart, Nus: Natural Reserve of Les Îles, 526 m, riparian forest, 11-26.VI.2013, 1 o", leg. Negro (MRSN-VDA); La Thuile: Comba Thuilette, $2000 \mathrm{~m}$, peat bog, 17.VIII.2012, 2 \%, leg. Paschetta \& Giuliano.

Literature. — Rossi \& Arnò 1995; [Isaia 2005]; [Rossi \& Bosio 2012].

Chorotype. - OLA.

MaCrOHABITAT. - Shrublands, wetlands, riparian habitats.

*Neriene emphana (Walckenaer, 1841)

NEW DATA. - Pont-Saint-Martin: Natural Reserve of Stagno di Holay, $702 \mathrm{~m}$, broadleaved forest (Castanea sativa), 11-26.VI.2013, 1 ㅇ, leg. Negro.

Chorotype. - PAL.

MACROHABITAT. - Broadleaved forests.

Notes. - New record for Aosta Valley.

Neriene peltata (Wider, 1834)

Literature. — Negro et al. 2009; [Rossi \& Bosio 2012].

Chorotype. - OLA.

Macrohabitat. - Coniferous forests.

- Oedothorax agrestis (Blackwall, 1853)

LiterATURE. - Calloni 1889 sub O. agrestis longipes (Simon, 1884); [Isaia 2005].

Chorotype. - ALPC.

Macrohabitat. - Not specified. 
Notes. - Old record in need of verification, also reported as doubtful by Rossi \& Bosio (2012). Calloni’s record originally refers to $O$. agrestis longipes, which was later synonymised by de Lessert (1910) with O. agrestis (Blackwall, 1853). The synonymy is currently overlooked in the World Spider Catalog.

*Oedothorax apicatus (Blackwall, 1850)

NEW DATA. - Arvier: Lago di Lolair, 1175 m, wet meadow, 14.VI5.VII.2011, 1 \%, leg. Paschetta \& Giuliano.

Chorotype. - PAL.

MACROHABITAT. - Wetlands.

Notes. - New record for Aosta Valley.

• Oedothorax gibbifer (Kulczyński, 1882)

LiterATURE. - Simon 1884; Pavesi 1904; [Isaia 2005]; [Rossi \& Bosio 2012].

CHOROType. - EUR.

MacrohabitaT. - Not specified.

NOTES. - Old record in need of verification.

Oedothorax retusus (Westring, 1851)

LiterATURE. — Rossi \& Arnò 1995; [Isaia 2005]; [Rossi \& Bosio 2012].

Chorotype. - PAL.

MACROHABITAT. - Shrublands.

-Oreoneta montigena (L. Koch, 1872)

LITERATURE. - Calloni 1889; [Isaia 2005].

Chorotype. - SEU.

MacrohabitaT. - Not specified.

Notes. - Old record in need of validation. The occurrence of the species in Aosta Valley is also considered doubtful by Rossi \& Bosio (2012).

\section{- Oreonetides glacialis (L. Koch, 1872)}

LiTerATURE. — Calloni 1889; [Pavesi 1904]; [Isaia 2005].

Chorotype. - EUR.

MacrohabitaT. - Not specified.

NOTES. - Old record in need of verification. The occurrence of the species in Aosta Valley is also considered doubtful by Rossi \& Bosio (2012).
-Oreonetides vaginatus (Thorell, 1872)

NEW DATA. — Ayas: Antagnod, 1970-2070 m, coniferous forests, leg. Franco \& Negro.

Literature. - Calloni 1889; Pavesi 1904; [Isaia 2005].

Chorotype. - OLA.

MacrohabitaT. - Coniferous forests.

Notes. - Old record in need of validation. The occurrence of the species in Aosta Valley was considered doubtful by Rossi \& Bosio (2012).

Palliduphantes pallidus (O. P.-Cambridge, 1871)

NeW DaTA. - Torgnon: Lo Ditor, 1950 m, peat bog, 12-26. VII.2013, 1 \%, leg. Negro.

Literature. - Isaia 2005; Negro et al. 2009; [Rossi \& Bosio 2012].

Chorotype. - EUR.

Macrohabitat. - Wetlands, alpine prairies.

Pelecopsis elongata (Wider, 1834)

Literature. - Negro et al. 2009; [Rossi \& Bosio 2012].

CHORotype. - EUR.

MacrohabitaT. - Coniferous forests.

*Pelecopsis parallela (Wider, 1834)

NeW DATA. - Brissogne, Saint-Marcel, Quart, Nus: Natural Reserve of Les Îles, $530 \mathrm{~m}$, dry meadow, 29.VII-18.VIII.2011, 1 o", $^{\prime \prime}$ leg. Paschetta \& Giuliano.

CHOROTYPE. - PAL.

MACrOHABITAT. - Shrublands.

Notes. - New record for Aosta Valley.

\section{Pelecopsis radicicola (L. Koch, 1872)}

NEW DATA. - Torgnon: Lo Ditor, 1950 m, peat bog, 12-26. VII.2013, 1 o", leg. Negro; same locality, 26.VI-12.VII.2013, $10^{\prime \prime}$ 1 \%, leg. Negro; Ayas: Antagnod, 1970-2070 m, coniferous forests, leg. Franco \& Negro.

Literature. - Negro et al. 2009; [Rossi \& Bosio 2012].

CHOrotype. - SIE.

MaCrohabitaT. - Coniferous forests, wetlands.

Peponocranium orbiculatum (O. P.-Cambridge, 1882)

Literature. - Negro et al. 2009; [Rossi \& Bosio 2012]. 
Chorotype. - CEU.

Macrohabitat. - Conifer forests.

Pityohyphantes phrygianus (C. L. Koch, 1836)

Literature. — Negro et al. 2009; [Rossi \& Bosio 2012].

CHOrotype. - PAL.

MACROHABITAT. - Coniferous forests.

*Pocadicnemis juncea Locket \& Millidge, 1953

NEW DATA. - Arvier: Lago di Lolair, 1175 m, wet meadow, 14.VI5.VII.2011, 1 ㅇ, leg. Paschetta \& Giuliano (MRSN-VDA); same locality, 14.VI-5.VII.2011, 1 ơ, leg. Paschetta \& Giuliano; same locality, 5-29.VII.2011, 1 \%, leg. Paschetta \& Giuliano; same locality, 29.VII-18.VIII.2011, 1 \%, leg. Paschetta \& Giuliano; Brissogne, Saint-Marcel, Quart, Nus: Natural Reserve of Les Îles, 530 m, riparian vegetation, 14.VI-5.VII.2011, 1 \%, leg. Paschetta \& Giuliano; Morgex-La Salle: Natural Reserve of Marais, 896 m, grove of reeds, 26.VI-12.VII.2013, 1 \% , leg. Negro.

Chorotype. - ASE.

MACROHABITAT. - Wetlands, riparian habitats.

Notes. - New record for Aosta Valley.

*Pocadicnemis pumila (Blackwall, 1841)

NEW DATA. - Courmayeur: Val Ferret, 1630 m, shrubs, 26.VI-12. VII.2013, 5 ơ 3 ㅇ, leg. Negro; same locality, 14-28.VIII.2013, 1 웅, leg. Negro; Morgex-La Salle: Natural Reserve of Marais, 896 m, grove of reeds, 11-26.VI.2013, 1 o", leg. Negro (MRSN-VDA); same locality, 11-26.VI.2013, 4 o", leg. Negro; same locality, 26.VI-12. VII.2013, $10^{7} 1$ \% , leg. Negro; same locality, grove of reeds, 26.VII14.VIII.2013, leg. Negro.

Chorotype. - OLA.

MACrOHABITAT. - Shrublands, wetlands, riparian habitats.

Notes. - New record for Aosta Valley. Rossi \& Arnò (1995) reported Pocadicnemis prope pumila for the Natural Reserve of Les Iles. Accordingly, Rossi \& Bosio (2012) reported the same citation for this species as doubtful.

Porrhomma microphthalmum (O. P.-Cambridge, 1871)

LiterATURE. - Negro et al. 2009; Isaia et al. 2011; [Rossi \& Bosio 2012]

Chorotype. - PAL.

MacrohabitaT. - Caves.

*Porrhomma pygmaeum (Blackwall, 1834)

NEW DATA. - Pont-Saint-Martin: Natural Reserve of Stagno di Holay, $775 \mathrm{~m}$, riparian vegetation, 5.IV.2012, 1 \%, leg. Paschetta \& Isaia.
Chorotype. - ASE.

MACROHABITAT. - Riparian habitats.

Notes. - New record for Aosta Valley.

*Prinerigone vagans (Audouin, 1826)

NEW DATA. - Fontainemore, Monte Mars, 1900 m, peat bog, 14.IX.2012, 1 \%, leg. Paschetta \& Mammola; same locality, 17.IX.2012, 2 \&, leg. Paschetta \& Marguerettaz; La Thuile: Comba Thuilette, $2000 \mathrm{~m}$, peat bog, 10.VII.2012, 1 \%, leg. Paschetta, Giuliano, Mammola \& Marguerettaz.

Chorotype. - PAL.

MACROHABITAT. - Wetlands.

Notes. - New record for Aosta Valley.

\section{*Scotinotylus alpigena (L. Koch, 1869)}

NEW DATA. — Ayas: Antagnod, 1970-2070 m, coniferous forests, leg. Franco \& Negro.

Chorotype. - ASE.

Macrohabitat. - Coniferous forests.

Notes. - New record for Aosta Valley.

•Scotinotylus antennatus (O. P.-Cambridge, 1875)

Literature. — Calloni 1889; [Pavesi 1904]; [Isaia 2005].

Chorotype. - CEU.

MacrohabitaT. - Not specified.

Notes. - Old record in need of validation. The occurrence of the species in Aosta Valley is also considered doubtful by Rossi \& Bosio (2012).

Stemonyphantes lineatus (Linnaeus, 1758)

Literature. — Rossi \& Arnò 1995; [Isaia 2005]; [Rossi \& Bosio 2012].

Chorotype. - PAL.

MACROHABITAT. - Shrublands.

*Tapinocyba insecta (L. Koch, 1869)

NEW DATA. - Brissogne, Saint-Marcel, Quart, Nus: Natural Reserve of Les Îles, $530 \mathrm{~m}$, riparian vegetation, 14.VI-5.VII.2011, 2 일. leg. Paschetta \& Giuliano; same locality, dry meadow, 14.VI5.VII.2011, 1 o , leg. Paschetta \& Giuliano; same locality, 5-29. VII.2011, 1 ㅇ, leg. Paschetta \& Giuliano.

CHOrotype. - EUR. 
MACROHABITAT. - Riparian habitats, shrublands.

Notes. - New record for Aosta Valley.

\section{Tapinocyba maureri Thaler, 1991}

Literature. - Pesarini 2000; [Isaia 2005]; [Rossi \& Bosio 2012].

Chorotype. - ALPC.

MACROHABITAT. - Not specified.

Tapinocyba pallens (O. P.-Cambridge, 1872)

Literature. - Negro et al. 2009; [Rossi \& Bosio 2012].

Chorotype. - SIE.

MaCrOHABITAT. - Alpine prairies.

\section{Tenuiphantes flavipes (Blackwall, 1854)}

NEW DATA. - Pont-Saint-Martin: Natural Reserve of Stagno di Holay, 775 m, riparian vegetation, 5.IV.2012, 2 \& , leg. Paschetta \& Isaia; same locality, 13.VIII.2012, 1 o", leg. Paschetta; same locality, broadleaved forest (Castanea sativa), 26.VII-14.VIII.2013, $10^{\circ}$, leg. Negro; Morgex-La Salle: Natural Reserve of Marais, 896 m, grove of reeds, 26.VI-12.VII.2013, $20^{\prime \prime} 1 \%$, leg. Negro; same locality, 26.VII-14.VIII.2013, 2 o", leg. Negro; same locality, riparian forest, 12-26.VII.2013, 1 ơ, leg.Negro; Arvier: Lago di Lolair, 1175 m, wet meadow, 14.VI-5.VII.2011, 1 o', leg. Paschetta \& Giuliano; Saint Denis: Brison-Cly, 1000 m, dry meadow, 4-25. VII.2012, 1 \&, leg. Negro (MRSN-VDA); Challand-Saint-Victor: Natural Reserve of Lago di Villa, $820 \mathrm{~m}$, broadleaved forest, 26.VII14.VIII.2013, 1 ơ $^{7}$ leg. Negro; Saint Christophe: Tsatelet, 750 m, broadleaved forest, 13.VI-4.VII.2012, 1 \% leg. Negro; Torgnon: Lo Ditor, 1950 m, peat bog, 26.VII-14.VIII.2013, 1 o", leg. Negro; same locality, 11-26.VI.2013, 1 ㅇ, leg. Negro; Gressan: Natural Reserve of Côte de Gargantua, 700 m, broadleaved forest, 4-25. VII.2012, 1 \%, leg. Negro.

Literature. - Isaia 2005; Negro et al. 2009; [Rossi \& Bosio 2012].

Chorotype. - PAL.

MACROHABitaT. - Riparian habitats, wetlands, broadleaved forests, shrublands.

\section{*Tenuiphantes mengei (Kulczyński, 1887)}

NEW DATA. - Morgex-La Salle: Natural Reserve of Marais, 900 m, wet meadow, 14.VI-5.VII.2011, 1 ơ 1 \%, leg. Paschetta \& Giuliano; same locality, 5-29.VII.2011, 2 ơ 2 ㅇ, leg. Paschetta Giuliano; same locality, riparian forest, 14.VI-5.VII.2011, $20^{7} 4$ o , leg. Paschetta \& Giuliano; same locality, 5-29.VII.2011, 3 o, leg. Paschetta, Giuliano; same locality, meadow/grove of reeds, 9.VII.2012, 1 ㅇ, leg. Paschetta \& Giuliano.

Chorotype. - PAL.

MACROHABITAT. - Wetlands, riparian habitats.

Notes. - New record for Aosta Valley.
Tenuiphantes tenebricola (Wider, 1834)

NEW DATA. - Torgnon: Lo Ditor, 1950 m, peat bog, 26.VII-14. VIII.2013, 1 \%, leg. Negro.

Literature. - Pavesi 1904; [Isaia 2005]; De Angelis \& Fantoni 2008; [Rossi \& Bosio 2012].

CHorotype. - SIE.

MACROHABITAT. - Wetlands.

Tenuiphantes tenuis (Blackwall, 1852)

Literature. - Isaia 2005; Negro et al. 2009; [Rossi \& Bosio 2012].

Chorotype. - TUE.

Macrohabitat. - Coniferous forests, alpine prairies.

Tiso vagans (Blackwall, 1834)

Literature. - Negro et al. 2009; [Rossi \& Bosio 2012].

ChOrotype. - EUR.

MACROHABITAT. - Coniferous forests, alpine prairies.

* Trichoncus sordidus Simon, 1884

NEW DATA. - Challand-Saint-Victor: Natural Reserve of Lago di Villa, 820 m, meadow, 11-26.VI.2013, 1 o", leg. Negro (MRSN-VDA).

Chorotype. - SEU.

MACROHABITAT. - Alpine grasslands.

Notes. - New record for Aosta Valley.

Trichopterna cito (O. P.-Cambridge, 1872)

Literature. - Negro et al. 2009; [Rossi \& Bosio 2012].

Chorotype. - PAL.

Macrohabitat. - Coniferous forests, alpine prairies.

\section{Troglohyphantes lucifuga (Simon, 1884)}

(Fig. 7)

NEW DATA. — Chabodey, Borna d'la Glace, 1545 m, cave, 16.IX.2014, 3 ơ, leg. Mammola \& Isaia; Verrogne, Fessura di Verrogne, 1536 m, cave, 16.IX.2014, 1 ơ, leg. Mammola \& Isaia.

Literature. - Simon 1884; Pavesi 1904; Brignoli 1979; Arnò \& Lana 2005; [Isaia 2005]; Isaia et al. 2011; [Rossi \& Bosio 2012].

Chorotype. - ALPW.

Macrohabitat. - Caves. 
NoteS. - In keeping with Simon (1884) and according to Isaia et al. (2010), the record of T. sordellii reported by Pavesi (1904) (Saint Rhémy, Grand Saint Bernard) has to be assigned to T. lucifuga. T. sordellii is an endemic species of Orobian Alps and Prealps of Bergamo, Prealps of Brescia and Prealps of Lugano.

Troglohyphantes nigraerosae Brignoli, 1971

LiTERATURE. — Brignoli 1971b; [Isaia 2005].

Chorotype. - ALPW.

MaCrOHABITAT. - Rocky lands.

NoTES. - In the original description, Brignoli (1971b) reports as type locality "Colle dell'Arietta", which marks the border between Piedmont and Aosta Valley. The occurrence of this species in Aosta Valley is considered doubtful by Rossi \& Bosio (2012).

Troglohyphantes sarae Pesarini, 2011

Literature. — Pesarini 2011; [Rossi \& Bosio 2012].

Chorotype. - ALPW.

MacrohabitAT. - Alpine prairies.

\section{Troxochrus scabriculus (Westring, 1851)}

NeW DATA. - Brissogne, Saint-Marcel, Quart, Nus: Natural Reserve of Les Îles, $530 \mathrm{~m}$, riparian forest, 29.VII-18.VIII.2011, $10^{\circ}$, leg. Paschetta \& Giuliano; same locality, 26.VI-12.VII.2013, $10^{7}$, leg. Negro; same locality, 12-26.VII.2013, 1 o", leg. Negro.

Literature. - Rossi \& Arnò 1995; [Isaia 2005]; [Rossi \& Bosio 2012].

Chorotype. - SIE.

MACROHABITAT. - Riparian habitats.

-Typhocrestus digitatus (O. P.-Cambridge, 1872)

Literature. — Calloni 1889; [Pavesi 1904]; [Isaia 2005].

CHorotype. - SIE.

MACROHABITAT. - Not specified.

NOTES. - Old record in need of validation. The occurrence of the species in Aosta Valley is also considered doubtful by Rossi \& Bosio (2012).

Walckenaeria acuminata Blackwall, 1833

NEW DATA. - Brissogne, Saint-Marcel, Quart, Nus: Natural Reserve of Les Îles, 530 m, dry meadow, 14.VI-5.VII.2011, 1 \%, leg. Paschetta \& Giuliano.

Literature. - De Angelis \& Fantoni 2008; [Rossi \& Bosio 2012].

Chorotype. - EUR.

MACROHABITAT. — Shrublands.

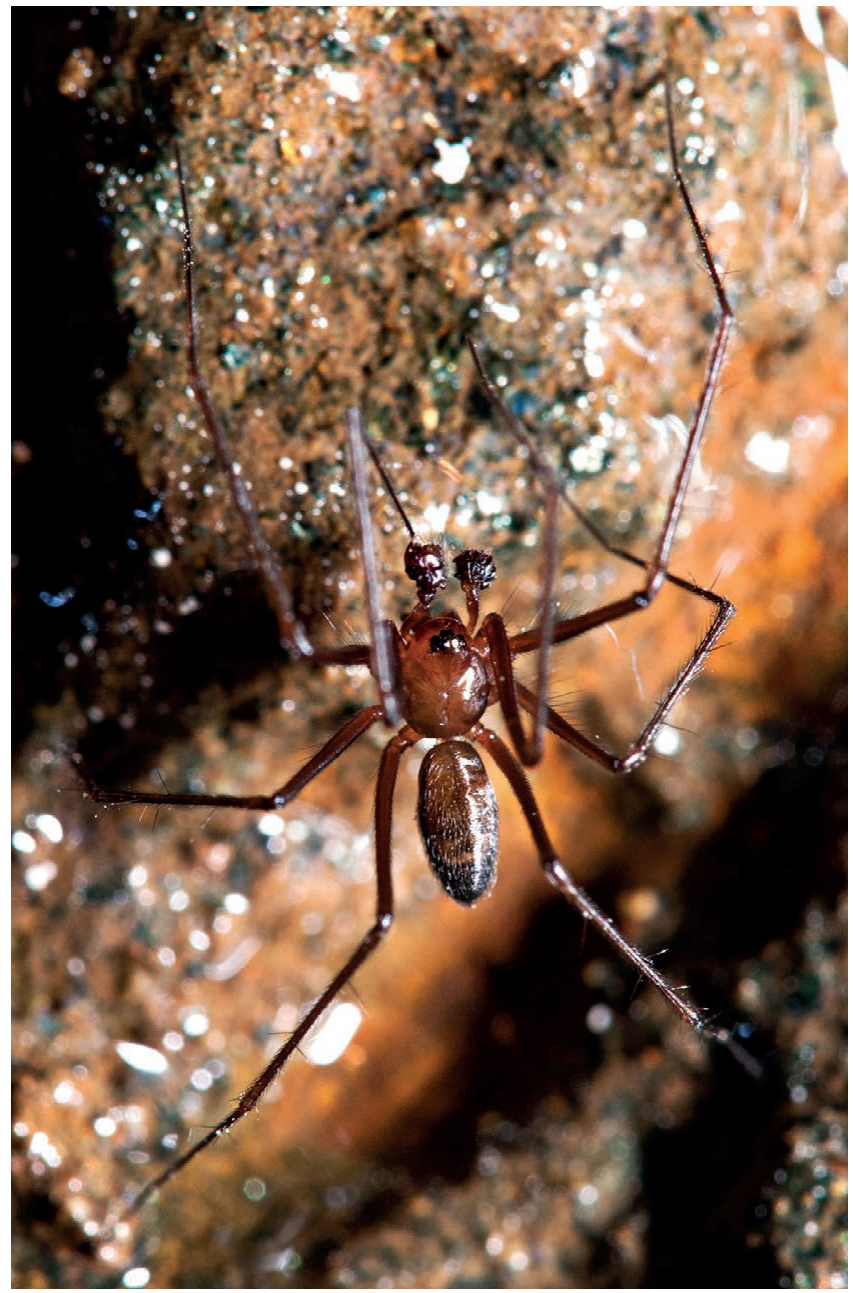

FIG. 7. - Troglohyphantes lucifuga (Simon, 1884) (Linyphiidae), male. Average body length: $6.8 \mathrm{~mm}$. Chabodey: Borna d'la Glace. Photograph: Mauro Paschetta 2014

Walckenaeria alticeps (Denis, 1952)

NEW DATA. - Torgnon: Lo Ditor, 1950 m, peat bog, 26.VI-12. VII.2013, 1 ơ, leg. Negro.

Literature. — Negro et al. 2009; [Rossi \& Bosio 2012].

CHOrotype. - EUR.

MACROHABITAT. - Wetlands, alpine prairies.

\section{Walckenaeria antica (Wider, 1834)}

NEW DATA. - Pont-Saint-Martin: Natural Reserve of Stagno di Holay, 616 m, meadow, 11-26.VI.2013, 1 ㅇ, leg. Negro (MRSN-VDA); Ayas: Antagnod, 1970-2070 m, alpine grassland, leg. Franco \& Negro.

Literature. — Gobbi et al. 2010; [Rossi \& Bosio 2012].

Chorotype. - ASE.

MACROHABITAT. - Alpine grasslands, rocky lands. 
Walckenaeria atrotibialis (O. P.-Cambridge, 1878)

Literature. - Negro et al. 2009; [Rossi \& Bosio 2012].

Chorotype. - OLA.

MacrohabitaT. - Coniferous forests, alpine prairies.

\section{Walckenaeria furcillata (Menge, 1869)}

NEW DATA. - Saint Christophe: Tsatelet, $750 \mathrm{~m}$, broadleaved forest, 13.VI-4.VII.2012, 1 o', leg. Negro (MRSN-VDA).

Literature. — Negro et al. 2009; [Rossi \& Bosio 2012].

Chorotype. - PAL.

Macrohabitat. - Broadleaved forests, alpine prairies.

\section{-Walckenaeria monoceros (Wider, 1834)}

Literature. - Calloni 1889; [Isaia 2005].

Chorotype. - TUE.

MacrohabitaT. - Not specified.

Notes. - Old record in need of validation. The occurrence of the species in Aosta Valley is also considered doubtful by Rossi \& Bosio (2012).

Walckenaeria nodosa O. P.-Cambridge, 1873

Literature. — Negro et al. 2009; [Rossi \& Bosio 2012]. Chorotype. - PAL.

Macrohabitat. - Coniferous forests, alpine prairies.

Walckenaeria obtusa Blackwall, 1836

Literature. — Negro et al. 2009; [Rossi \& Bosio 2012].

Chorotype. - PAL.

Macrohabitat. - Coniferous forests, alpine prairies.

•Walckenaeria vigilax (Blackwall, 1853)

LiterATURE. — Calloni 1889; [Pavesi 1904]; [Isaia 2005]

Chorotype. - OLA.

MACROHABITAT. - Not specified.

Notes. - Old record in need of validation. The occurrence of the species in Aosta Valley is also considered doubtful by Rossi \& Bosio (2012).
Family LIOCRANIDAE Simon, 1897

Agroeca cuprea Menge, 1873

NEW DATA. - Brissogne, Saint-Marcel, Quart, Nus: Natural Reserve of Les Îles, 530 m, dry meadow, 14.VI-5.VII.2011, 6 \% , leg. Paschetta \& Giuliano; same locality, 5-29.VII.2011, 3 \%, leg. Paschetta \& Giuliano; same locality, 18.VIII-16.IX.2011, 1 \% , leg. Paschetta \& Giuliano; same locality, riparian vegetation, 14.VI-5.VII.2011, 1 , leg. Paschetta \& Giuliano; same locality, 5-29.VII.2011, 8 ㅇ, leg. Paschetta \& Giuliano; same locality, 29.VII-18.VIII.2011, 3 \%, leg. Paschetta \& Giuliano; same locality, riparian forest, 26.VI-12. VII.2013, 3 \% , leg. Negro; same locality, 12-26.VII.2013, 1 \% leg. Negro; same locality, dry meadow, 26.VII-14.VIII.2013, 1 \%, leg. Negro; Saint Christophe: Tsatelet, $750 \mathrm{~m}$, broadleaved forest, 3-24.V.2012, 1 9, leg. Negro; same locality, 4-25.VII.2012, 1 ㅇ, leg. Negro; Challand-Saint-Victor: Natural Reserve of Lago di Villa, 820 m, wet meadow, 13.VI-4.VII.2011, 1 \%, leg. Paschetta \& Giuliano (MRSN-VDA); same locality, broadleaved forest, 26.VI12.VII.2013, 1 \%, leg. Negro; Pont-Saint-Martin: Natural Reserve of Stagno di Holay, 616 m, alpine grassland, 11-26.VI.2013, 1 \%, leg. Negro; same locality, 26.VII-14.VIII.2013, 1 \%, leg. Negro; same locality, broadleaved forest (Castanea sativa), 11-26.VI.2013, $1 \%$, leg. Negro; Morgex-La Salle: Natural Reserve of Marais, 900 m, wet meadow, 29.VII-18.VIII.2011, 1 ㅇ, leg. Paschetta \& Giuliano; same locality, 14.VI-5.VII.2011, 1 \%, leg. Paschetta \& Giuliano; same locality, riparian forest, 26.VII-14.VIII.2013, 1 \&, leg. Negro; Aymavilles: Pont d'Ael, $800 \mathrm{~m}$, broadleaved forest, 3-24.V.2012, 1 \%, leg. Negro (MNHN); Ayas: Champoluc, 1650-1850 m, ski pistes, 2007, leg. Franco \& Negro.

Literature. - Negro et al. 2009, 2010; [Rossi \& Bosio 2012].

Chorotype. - ASE.

MACROHABITAT. - Riparian habitats, shrublands, broadleaved forests, wetlands, alpine grasslands, ski pistes.

\section{Agroeca proxima (O. P.-Cambridge, 1871)}

NeW DATA. - Torgnon: Lo Ditor, 1950 m, peat bog, 26.VII-14. VIII.2013, $10^{\star}$, leg. Negro; Courmayeur: Val Ferret, 1630 m, shrubs, 14-28.VIII.2013, 2 o' 1 \%, leg. Negro; Morgex-La Salle: Natural Reserve of Marais, 896 m, grove of reeds, 28.VIII-16.IX.2013, 1 ㅇ, leg. Negro; Arvier: Lago di Lolair, 1175 m, wet meadow, 18.VIII-16. IX.2011, $10^{7}$, leg. Paschetta \& Giuliano; Ayas: Antagnod, 19702070 m, coniferous forests, 2007, leg. Franco \& Negro.

Literature. - De Angelis \& Fantoni 2008; [Rossi \& Bosio 2012]; Negro et al. 2013.

CHOROTYPe. - SIE.

MACROHABITAT. - Wetlands, shrublands, coniferous forests, alpine prairies, ski pistes.

\section{Liocranum rupicola (Walckenaer, 1830)}

NEW DATA. - Saint Denis: Brison-Cly, 1000 m, dry meadow, 4-25. VII.2012, 1 ơ, leg. Negro, (MRSN-VDA); Pont-Saint-Martin: Natural Reserve of Stagno di Holay, $679 \mathrm{~m}$, broadleaved forest (Castanea sativa), 11-26.VI.2013, 1 O", leg. Negro; Saint Pierre: Monte Torretta, $900 \mathrm{~m}$, broadleaved forest, 24.V-13.VI.2012, 1 \% leg. Negro (MRSN-VDA). 
Literature. - Rossi \& Bosio 2012.

CHOrotype. - EUR.

MACROHABITAT. - Broadleaved forests, shrublands, synanthropic habitats.

\section{*Mesiotelus tenuissimus (L. Koch, 1866)}

NEW DATA. - Sarre-Saint Pierre: Monte Torretta, 900 m, broadleaved forest, 3-24.V.2012, 2 o", leg. Negro; Aymavilles: Pont d'Ael, $800 \mathrm{~m}$, broadleaved forest, 3-24.V.2012, 1 ơ, leg. Negro.

Chorotype. - MED.

MACROHABITAT. - Broadleaved forests.

Notes. - New record for Aosta Valley.

\section{Scotina celans (Blackwall, 1841)}

Literature. - De Angelis \& Fantoni 2008; Negro et al. 2009; [Rossi \& Bosio 2012].

Chorotype. - EUR.

Macrohabitat. - Coniferous forests, alpine prairies.

Family LyCOSIDAE Sundevall, 1833

Alopecosa accentuata (Latreille, 1817)

NEW DATA. - Ayas: Antagnod, 1970-2070 m, alpine pasture, 2007, leg. Franco \& Negro; Aymavilles: Pont d'Ael, 800 m, dry meadow, 13.VI-4.VII.2012, 1 \%, leg. Negro (MRSN-VDA); same locality, 24.V-13.VI.2012, 1 \% , leg. Negro (MNHN); Torgnon: Lo Ditor, 1950 m, peat bog, 11-26.VI.2013, 1 o $^{\prime \prime}$ leg. Negro; Sarre-Saint Pierre: Monte Torretta, 900 m, broadleaved forest, 3-24.V.2012, 1 , leg. Negro.

LiTERATURE. - Lugetti \& Tongiorgi 1969; [Isaia 2005]; De Angelis \& Fantoni 2008; Negro et al. 2009; [Rossi \& Bosio 2012].

Chorotype. - SIE.

MACROHABITAT. - Alpine pastures, shrublands, broadleaved forests, ski pistes, wetlands.

\section{Alopecosa aculeata (Clerck, 1757)}

NEW DATA. - Sarre-Saint Pierre: Monte Torretta, 900 m, dry meadow, 13.VI-4.VII.2012, 1 \%, leg. Negro (MRSN-VDA).

Literature. — Lugetti \& Tongiorgi 1969; [Isaia 2005]; Negro et al. 2009; [Rossi \& Bosio 2012].

Chorotype. - OLA.

MACROHABITAT. - Shrublands, coniferous forests, alpine prairies.

\author{
Alopecosa cuneata (Clerck, 1757)
}

NEW DATA. - Ayas: Antagnod, 1970-2070 m, ski pistes, leg. Franco \& Negro; Torgnon: Lo Ditor, 1950 m, peat bog, 11-26. VI.2013, 16 o' 5 \% , leg. Negro; same locality, 11-26.VI.2013, $10^{\prime \prime}$, leg. Negro (MRSN-VDA); same locality, 26.VI-12.VII.2013, 3 ơ, leg. Negro.

LiteraTURE. — Lugetti \& Tongiorgi 1969; [Isaia 2005]; De Angelis \& Fantoni 2008; Negro et al. 2009, 2013; [Rossi \& Bosio 2012].

Chorotype. - PAL.

MACrOHABITAT. - Wetlands, ski pistes, alpine prairies, alpine pastures.

\section{*Alopecosa cursor (Hahn, 1831)}

NEW DATA. - Gressan: Natural Reserve of Côte de Gargantua, 700 m, dry meadow, 3-24.V.2012, 1 o", leg. Negro, (MRSN-VDA); Aymavilles: Pont d'Ael, 800 m, broadleaved forest, 3-24.V.2012, $20^{7}$, leg. Negro (MNHN).

Chorotype. - PAL.

MACROHABITAT. - Shrublands, broadleaved forests.

Notes. - New record for Aosta Valley.

\section{Alopecosa fabrilis (Clerck, 1757)}

LiTERATURE. - Di Caporiacco 1928; Lugetti \& Tongiorgi 1969; [Isaia 2005]; [Rossi \& Bosio 2012].

Chorotype. - PAL.

MACROHABITAT. - Alpine pastures.

\section{Alopecosa pulverulenta (Clerck, 1757)}

New DATA. - Sarre-Saint Pierre: Monte Torretta, 900 m, dry meadow, 3-24.V.2012, $90^{7}$, leg. Negro; same locality, 24.V-13. VI.2012, $10^{7}$, leg. Negro; Morgex-La Salle: Natural Reserve of Marais, 896 m, grove of reeds, 26.VI-12.VII.2013, 1 , leg. Negro; same locality, riparian forest, 26.VI-12.VII.2013, 2 \% leg. Negro; same locality, 11-26.VI.2013, $10^{\top} 3$ \%, leg. Negro; Torgnon: Lo Ditor, 1950 m, peat bog, 11-26.VI.2013, 10 o 2 \%, leg. Negro; same locality, 26.VI-12.VII.2013, $60^{7} 3$ \% , leg. Negro; same locality, 12-26.VII.2013, 2 o' 1 \%, leg. Negro; same locality, 26.VII-14. VIII.2013, 1 ơ 2 \%, leg. Negro; Aymavilles: Pont d'Ael, 800 m, dry meadow, 24.V-13.VI.2012, $20^{\prime \prime}$, leg. Negro; Courmayeur: Val Ferret, 1630 m, shrubs, 26.VI-12.VII.2013, 5 ơ 3 \%, leg. Negro; same locality, $1740 \mathrm{~m}$, gravel bed of a river, 14-28.VIII.2013, $2 \mathrm{O}^{\text {" }}$ $1 \%$, leg. Negro; Challand-Saint-Victor: Natural Reserve of Lago di Villa, 820 m, meadow, 11-26.VI.2013, 3 , leg. Negro; same locality, 26.VI-12.VII.2013, 5 \& , leg. Negro; same locality, broadleaved forest, 12-26.VII.2013, 1 \%, leg. Negro; Gressan: Natural Reserve of Côte de Gargantua, 700 m, dry meadow, 3-24.V.2012, 1 o", leg. Negro; Pont-Saint-Martin: Natural Reserve of Stagno di Holay, $775 \mathrm{~m}$, wet meadow, 5.IV.2012, $10^{7}$, leg. Paschetta \& Isaia; same locality, 11-26.VI.2013, 3 ㅇ, leg. Negro; same locality, 26.VI-12. VII.2013, 1 ơ 1 \& , leg. Negro. 
Literature. - Calloni 1889; Pavesi 1904; di Caporiacco 1928; Lugetti \& Tongiorgi 1969; Rossi \& Arnò 1995; [Isaia 2005]; De Angelis \& Fantoni 2008; [Rossi \& Bosio 2012].

Chorotype. - PAL.

MACROHABITAT. - Wetlands, riparian habitats, gravel beds, shrublands, broadleaved forests, alpine grasslands.

\section{Alopecosa sulzeri (Pavesi, 1873)}

NEW DATA. - Aymavilles: Pont d'Ael, $800 \mathrm{~m}$, broadleaved forest, 24.V-13.VI.2012, 1 o', leg. Negro; same locality, 13.VI-4. VII.2012, 1 o", leg. Negro; same locality, 25.VII-20.VIII.2012, 1 \%, leg. Negro; same locality, dry meadow, 24.V-13.VI.2012, $100^{\top} 1$ O , leg. Negro; same locality, 24.V-13.VI.2012, $10^{7}$, leg. Negro (MRSN-VDA); same locality, 13.VI-4.VII.2012, $10^{7} 1$ o , leg. Negro; same locality, 4-25.VII.2012, 1 ㅇ, leg. Negro (MRSN-VDA); Sarre-Saint Pierre: Monte Torretta, $900 \mathrm{~m}$, dry meadow, 24.V-13.VI.2012, 1 \%, leg. Negro; Saint Denis: Brison-Cly, $1000 \mathrm{~m}$, broadleaved forest, 24.V-13.VI.2012, $30^{7} 1$ ㅇ, leg. Negro; same locality, 13.VI-4.VII.2012, $10^{7} 1$ 운, leg. Negro; same locality, 4-25.VII.2012, 1 o", leg. Negro; same locality, dry meadow, 24.V-13.VI.2012, 1 ㅇ, leg. Negro; same locality, 13.VI-4.VII.2012, 1 o", leg. Negro; same locality, 4-25. VII.2012, 1 o', leg. Negro.

Literature. — Gobbi et al. 2010; [Rossi \& Bosio 2012].

Chorotype. - EUR.

MACROHABITAT. - Broadleaved forests, shrublands, rocky lands.

\section{*Alopecosa taeniata (C. L. Koch, 1835)}

NEW DATA. - Ayas: Antagnod, 1970-2070 m, alpine pastures, 2007, leg. Franco \& Negro; same locality, coniferous forests, 2007 , leg. Franco \& Negro; Ayas: Champoluc, 1650-1850 m, ski pistes, 2007, leg. Franco \& Negro.

CHorotype. - SIE.

MACROHABITAT. - Ski pistes, alpine pastures, coniferous forests.

Notes. - New record for Aosta Valley.

\section{Alopecosa trabalis (Clerck, 1757)}

NEW DATA. - Ayas: Antagnod, 1970-2070 m, alpine pasture, 2007, leg. Franco \& Negro; same locality, ski pistes, 2007, leg. Franco \& Negro.

Literature. — Pavesi 1904; [Isaia 2005].

Chorotype. - TUE.

MACROHABITAT. - Alpine pastures, ski pistes.

\section{Arctosa figurata (Simon, 1876)}

NEW DATA. - Ayas: Antagnod, 1970-2070 m, alpine pasture, 2007, leg. Franco \& Negro.
Literature. - De Angelis \& Fantoni 2008; Negro et al. 2009; [Rossi \& Bosio 2012].

Chorotype. - EUR.

MACROHABITAT. - Alpine pastures.

\section{Arctosa leopardus (Sundevall, 1833)}

NEW DATA. - Courmayeur: Val Ferret, 1630 m, shrubs, 14-28. VIII.2013, 1 \%, leg. Negro; Verrayes: stagno di Loson, 1520 m, grove of reeds/peat bog, 13.VI-4.VII.2011, $60^{71} 1$ \%, leg. Paschetta $\&$ Giuliano; same locality, 4-28.VII.2011, $10^{\prime \prime} 6$ \% , leg. Paschetta \& Giuliano; same locality, 28.VII-17.VIII.2011, $10^{7} 2$ \% , leg. Paschetta \& Giuliano; Challand-Saint-Victor: Natural Reserve of Lago di Villa, 820 m, grove of reeds, 18.VIII-15.IX.2011, 2 \%, leg.Paschetta \& Giuliano (MRSN-VDA); Arvier: Lago di Lolair, 1175 m, wet meadow, 18.VIII-16.IX.2011, 1 \% , leg. Paschetta \& Giuliano; same locality, 29.VII-18.VIII.2011, 1 ơ', leg. Paschetta \& Giuliano.

Literature. — De Angelis \& Fantoni 2008; [Rossi \& Bosio 2012].

Chorotype. - ASE.

MACROHABITAT. - Shrublands, wetlands.

\section{*Arctosa lutetiana (Simon, 1876)}

NEW DATA. - Pont-Saint-Martin: Natural Reserve of Stagno di Holay, $702 \mathrm{~m}$, broadleaved forest (Castanea sativa), 11-26.VI.2013, $10^{x}$, leg. Negro (MRSN-VDA).

CHOrotype. - EUR.

MACROHABITAT. - Broadleaved forests.

Notes. - New record for Aosta Valley.

\section{-Arctosa perita (Latreille, 1799)}

Literature. — Pesarini 1997; [Isaia 2005].

Chorotype. - OLA.

MacrohabitAT. - Not specified.

Notes. - See notes about Hypsosinga heri. The occurrence of this species in Aosta Valley is also considered doubtful by Rossi \& Bosio (2012).

\section{*Arctosa personata (L. Koch, 1872)}

New DATA. - Aymavilles: Pont d'Ael, 800 m, dry meadow, 3-24.V.2012, 1 \%, leg. Negro; same locality, 3-24.V.2012, $10^{\text {", }}$ leg. Negro (MRSN-VDA); same locality, 24.V-13.VI.2012, 1 ơ 1 ㅇ, leg. Negro; same locality, 13.VI-4.VII.2012, 1 ơ 1 \%, leg. Negro; same locality, 4-25.VII.2012, 1 \% , leg. Negro; same locality, 25.VII-20. VIII.2012, 1 \%, leg. Negro; Challand-Saint-Victor: Natural Reserve of Lago di Villa, 820 m, meadow, 12-26.VII.2013, 1 ㅇ, leg. Negro; Gressan: Natural Reserve of Côte de Gargantua, 700 m, dry meadow, 24.V-13.VI.2012, $10^{7}$, leg. Negro (MNHN); Sarre-Saint Pierre: Monte Torretta, 900 m, dry meadow, 3-24.V.2012, 3 ơ 1 , 
leg. Negro; same locality, 24.V-13.VI.2012, 1 \% , leg. Negro; same locality, broadleaved forest, 25.VII-20.VIII.2012, 1 \% , leg. Negro.

Chorotype. - MED.

MACROHABITAT. — Shrublands, alpine grasslands, broadleaved forests.

Notes. - New record for Aosta Valley.

\section{Arctosa renidescens Buchar \& Thaler, 1995}

NEW DATA. - La Thuile: Comba Thuilette, 2000 m, peat bog, 10.VII.2012, 1 ơ, leg. Paschetta, Giuliano, Mammola \& Marguerettaz; Ayas: Antagnod, 1970-2070 m, alpine pasture, 2007, leg. Franco \& Negro; same locality, alpine prairies, 2007, leg. Franco \& Negro; same locality, ski pistes, 2007, leg. Franco \& Negro.

Literature. — Negro et al. 2009; [Rossi \& Bosio 2012].

Chorotype. - ALP.

Macrohabitat. - Ski pistes, broadleaved forests, wetlands, coniferous forests.

\section{Aulonia albimana (Walckenaer, 1805)}

NeW DATA. - Pont-Saint-Martin: Natural Reserve of Stagno di Holay, 616 m, alpine grassland, 11-26.VI.2013, 10 o $^{7} 1$ ㅇ, leg. Negro (MRSN-VDA); Ayas: Antagnod, 1970-2070 m, alpine pasture, 2007, leg. Franco \& Negro; Ayas: Antagnod, 1970-2070, ski pistes, 2007, leg. Franco \& Negro.

Literature. - Pavesi 1904; [Isaia 2005].

CHOrotype. - EUR.

MACROHABITAT. - Alpine grasslands, alpine pastures, ski pistes.

Notes. - The occurrence of this species in Aosta Valley was considered doubtful by Rossi \& Bosio (2012).

\section{Hogna radiata (Latreille, 1817)}

NEW DATA. - Challand-Saint-Victor: Natural Reserve of Lago di Villa, 820 m, wet meadow, 13.VI-4.VII.2011, 1 ᄋ, leg. Paschetta \& Giuliano; same locality, 28.VII-17.VIII.2011, 1 \%, leg. Paschetta \& Giuliano; same locality, 26.VII-14.VIII.2013, 1 ơ 1 \%, leg.Negro; Sarre-Saint Pierre: Monte Torretta, 900 m, dry meadow, 24.V13.VI.2012, 1 \% , leg. Negro; same locality, 4-25.VII.2012, 2 ㅇ, leg. Negro; same locality, 25.VII-20.VIII.2012, 1 \%, leg. Negro; Pont-Saint-Martin: Natural Reserve of Stagno di Holay, 775 m, wet meadow, 4-28.VII.2011, 1 o", leg. Paschetta \& Giuliano (MRSNVDA); same locality, 13.VIII.2012, 1 \%, leg. Paschetta; same locality, 18.VIII-15.IX.2011, 3 o", leg. Paschetta \& Giuliano; same locality, 26.VII-14.VIII.2013, 1 ơ, leg. Negro; same locality, 1428.VIII.2013, 1 o", leg. Negro; Saint Denis: Brison-Cly, $1000 \mathrm{~m}$, broadleaved forest, 4-25.VII.2012, 2 \%, leg. Negro; same locality, 4-25.VII.2012, 1 o", leg. Negro (MNHN); same locality, 25.VII20.VIII.2012, 1 \% , leg. Negro.

Literature. - Isaia 2005; [Rossi \& Bosio 2012].

Chorotype. - CAE.

MACROHABITAT. - Alpine grasslands, wetlands, shrublands, broadleaved forests.

\section{*Pardosa agrestis (Westring, 1861)}

NEW DATA. - Ayas: Antagnod, 1970-2070 m, alpine pasture, 2007, leg. Franco \& Negro; same locality, ski pistes, 2007, leg. Franco \& Negro.

Chorotype. - PAL.

Macrohabitat. - Ski pistes, alpine pastures.

Notes. - New record for Aosta Valley.

'Pardosa agricola (Thorell, 1856)

LiTERATURE. — Di Caporiacco 1928; [Isaia 2005]; [Rossi \& Bosio 2012].

CHOROTYPe. - SIE.

MACROHABITAT. - Alpine prairies.

Notes. - According to Tongiorgi (1966: 284, 311) and Pantini \& Isaia (2015), the species is not present in Italy despite the abundant citations in literature.

\section{Pardosa alacris (C. L. Koch, 1833)}

NEW DATA. - Ayas: Antagnod, 1970-2070 m, alpine pasture, 2007, leg. Franco \& Negro; same locality, ski pistes, 2007, leg. Franco \& Negro; Ayas: Champoluc, 1970-2070 m, ski pistes, 2007, leg. Franco \& Negro; Saint Christophe: Tsatelet, $750 \mathrm{~m}$, broadleaved forest, 3-24.V.2012, $130^{7} 5$ \% , leg. Negro; same locality, 24.V-13.VI.2012, 34 ơ 34 9, leg. Negro; same locality, 13.VI-4.VII.2012, 18 o' 36 \% leg. Negro; same locality, 25.VII20.VIII.2012, 3 ㅇ, leg. Negro; same locality, 4-25.VII.2012, 7 \%, leg. Negro; Sarre-Saint Pierre: Monte Torretta, 900 m, dry meadow, 13.VI-4.VII.2012, 1 ơ 3 \%, leg. Negro (MRSN-VDA); Brissogne, Saint-Marcel, Quart, Nus: Natural Reserve of Les Îles, $530 \mathrm{~m}$, riparian vegetation, 14.VI-5.VII.2011, 1 ơ $^{\prime \prime} 22$ \%, leg. Paschetta \& Giuliano; same locality, 5-29.VII.2011, $20^{7} 16$ ㅇ, leg. Paschetta \& Giuliano; same locality, 29.VII-18.VIII.2011, 2 ơ $^{\prime} 24$ 9 , leg. Paschetta \& Giuliano; same locality, 18.VIII-16. IX.2011, 15 9 , leg. Paschetta \& Giuliano; same locality, dry meadow, 29.VII-18.VIII.2011, 4 o , leg. Paschetta \& Giuliano; same locality, 5-29.VII.2011, 1 \%, leg. Paschetta \& Giuliano.

Literature. — Rossi \& Bosio 2012.

Chorotype. - EUR.

MACROHABITAT. - Broadleaved forests, shrublands, riparian habitats, ski pistes.

\section{Pardosa amentata (Clerck, 1757)}

(Fig. 8)

NEW DATA. - Courmayeur: Val Ferret, 1700 m, peat bog, 11.VII.2012, $20^{\text {T }} 6$ \% , leg. Paschetta Giuliano \& Mammola; same locality, grove of reeds, 11.VII.2012, 2 \% leg. Paschetta, Giuliano \& Mammola; same locality, 1779 m, shrubs, 14-28. VIII.2013, 3 ơ 9 잉 leg. Negro; same locality, shrubs, 26.VII14.VIII.2013, $10^{7} 10$ \% leg. Negro; same locality, 28.VIII-16. IX.2013, 2 \&, leg. Negro; Fontainemore, Monte Mars, 1900 m, peat bog, 14.IX.2012, 1 \% , leg. Paschetta \& Mammola; same locality, 
17.IX.2012, $20^{\pi} 79$, leg. Paschetta \& Marguerettaz; Morgex-La Salle: Natural Reserve of Marais, 900 m, riparian forest, 5-29. VII.2011, 1 ㅇ, leg. Paschetta, Giuliano; same locality, grove of reeds, 28.VIII-16.IX.2013, 2 \% , leg. Negro; La Thuile: Comba Thuilette, $2000 \mathrm{~m}$, peat bog, 10.VII.2012, $60^{n} 4$ 9 , leg. Paschetta, Giuliano, Mammola \& Marguerettaz; Verrayes: stagno di Loson, $1520 \mathrm{~m}$, grove of reeds/peat bog, 4-28.VII.2011, 1 ㅇ, leg. Paschetta \& Giuliano; Ayas: Champoluc, $1650-1850$ m, ski pistes, 2007, leg. Franco \& Negro.

Literature. - Calloni 1889; Pavesi 1904; di Caporiacco 1928; [Isaia 2005]; Negro et al. 2009; [Rossi \& Bosio 2012].

CHorotype. - SIE.

MACROHABITAT. - Wetlands, riparian habitats, ski pistes, shrublands.

\section{Pardosa bifasciata (C. L. Koch, 1834)}

New Data. - Aymavilles: Pont d'Ael, 800 m, dry meadow, 24.V13.VI.2012, 9 o' 3 \% , leg. Negro; same locality, 13.VI-4.VII.2012, $10^{7} 1 \%$, leg. Negro (MRSN-VDA); same locality, 4-25.VII.2012, 1 ㅇ, leg. Negro; same locality, 25.VII-20.VIII.2012, 1 ㅇ, leg. Negro; Brissogne, Saint-Marcel, Quart, Nus: Natural Reserve of Les Iles, $530 \mathrm{~m}$, dry meadow, 14.VI-5.VII.2011, $10^{7} 16$ \% , leg. Paschetta \& Giuliano; same locality, 5-29.VII.2011, 9 , leg. Paschetta \& Giuliano; same locality, 5-29.VII.2011, 8 \%, leg. Paschetta \& Giuliano; same locality, 18.VIII-16.IX.2011, 4 ㅇ, leg. Paschetta \& Giuliano; same locality, 29.VII-18.VIII.2011, 10 \& , leg. Paschetta \& Giuliano; same locality, 11-26.VI.2013, 1 \&, leg. Negro; Saint Denis: BrisonCly, 1000 m, broadleaved forest, 24.V-13.VI.2012, 5 o", leg. Negro; same locality, 13.VI-4.VII.2012, $60^{7} 8$ \% , leg. Negro; same locality, 4-25.VII.2012, 1 ơ 6 9, leg. Negro; same locality, 25.VII-20. VIII.2012, 1 오 , leg. Negro; Sarre-Saint Pierre: Monte Torretta, 900 m, dry meadow, 24.V-13.VI.2012, 1 ơ', leg. Negro; same locality, 13.VI-4.VII.2012, 1 o, leg. Negro; Challand-Saint-Victor: Natural Reserve of Lago di Villa, 820 m, meadow, 11-26.VI.2013, 10 o" leg. Negro; Arvier: Lago di Lolair, 1175 m, broadleaved forest, 24.V-13.VI.2012, 1 ơ, leg. Negro.

Literature. - Isaia 2005; De Angelis \& Fantoni 2008; Negro et al. 2009, 2010, 2013; [Rossi \& Bosio 2012].

Chorotype. - PAL.

MACROHABitaT. - Shrublands, broadleaved forests, alpine grasslands, ski pistes, alpine pastures.

\section{Pardosa blanda (C. L. Koch, 1833)}

NEW DATA. - Torgnon: Lo Ditor, 1950 m, peat bog, 11-26. VI.2013, 1 o', leg. Negro; same locality, 26.VI-12.VII.2013, 3 ơ $^{\prime \prime}$ 1 ㅇ, leg. Negro; same locality, 12-26.VII.2013, 17 ơ 4 우, leg. Negro; same locality, 26.VII-14.VIII.2013, $120^{7} 17$ \% leg. Negro; La Thuile: Comba Thuilette, 2000 m, peat bog, 10.VII.2012, 3 ㅇ, leg. Paschetta, Giuliano, Mammola \&Marguerettaz; same locality, 13.IX.2012, 3 \%, leg. Paschetta, Mammola \& Marguerettaz; Ayas: Antagnod, 1970-2070 m, alpine pasture, leg. Franco \& Negro; same locality, coniferous forest, leg. Franco \& Negro; same locality, ski pistes, leg. Franco \& Negro; Ayas: Champoluc, 1650-1850 m, ski pistes, leg. Franco \& Negro.

LITERATURE. - Calloni 1889; Tongiorgi 1966; Isaia 2000, [2005]; De Angelis \& Fantoni 2008; Negro et al. 2009, 2010, 2013; [Rossi \& Bosio 2012].
Chorotype. - SEU.

MACROHABITAT. - Ski pistes, alpine prairies, broadleaved forests, alpine pastures, wetlands, coniferous forests.

\section{Pardosa ferruginea (L. Koch, 1870)}

NEW DATA. — Ayas: Antagnod, 1970-2070 m, broadleaved forest, 2007, leg. Franco \& Negro.

Literature. - Negro et al. 2009; [Rossi \& Bosio 2012].

CHOROTYPe. - SIE.

MACROHABITAT. - Broadleaved forests, alpine prairies.

\section{Pardosa giebeli (Pavesi, 1873)}

LiterATURE. - Isaia 2005; [Rossi \& Bosio 2012].

Chorotype. - SEU.

Macrohabitat. - Not specified.

\section{Pardosa hortensis (Thorell, 1872)}

NEW DATA. - Morgex-La Salle: Natural Reserve of Marais, 896 m, riparian forest, 11-26.VI.2013, 1 \%, leg. Negro; (MRSN-VDA).

Literature. - Isaia 2005; [Rossi \& Bosio 2012].

Chorotype. - PAL.

MACROHABitaT. - Riparian habitats.

\section{Pardosa lugubris (Walckenaer, 1802)}

NEW DATA. - Morgex-La Salle: Natural Reserve of Marais, 900 m, riparian forest, 14.VI-5.VII.2011, 3 ơ 15 \%, leg. Paschetta \& Giuliano; same locality, 5-29.VII.2011, 2 \%, leg. Paschetta \& Giuliano; same locality, wet meadow, 29.VII-18.VIII.2011, 3 \% , leg. Paschetta \& Giuliano; same locality, 14.VI-5.VII.2011, 3 ơ, leg. Paschetta, Giuliano; same locality, 5-29.VII.2011, 3 , leg. Paschetta \& Giuliano; Arvier: Lago di Lolair, 1175 m, wet meadow, 14.VI-5.VII.2011, $10^{7}$, leg. Paschetta \& Giuliano.

Literature. - Pavesi 1904; Rossi \& Arnò 1995; Isaia 2000, [2005]; De Angelis \& Fantoni 2008; Negro et al. 2009; [Rossi \& Bosio 2012].

Chorotype. - PAL.

MACROHABITAT. - Riparian habitats, wetlands, shrublands, ski pistes, coniferous forests, broadleaved forests.

NOTES. - Given the uncertainty in species identification (see TöpferHofmann et al. 2000), old records should be treated as P. gr. lugubris.

\section{Pardosa mixta (Kulczyński, 1887)}

NEW DATA. - Courmayeur: Val Ferret, 1779 m, shrubs, 26.VI-12. VII.2013, 1 o", leg. Negro; La Thuile: Comba Thuilette, 2000 m, 


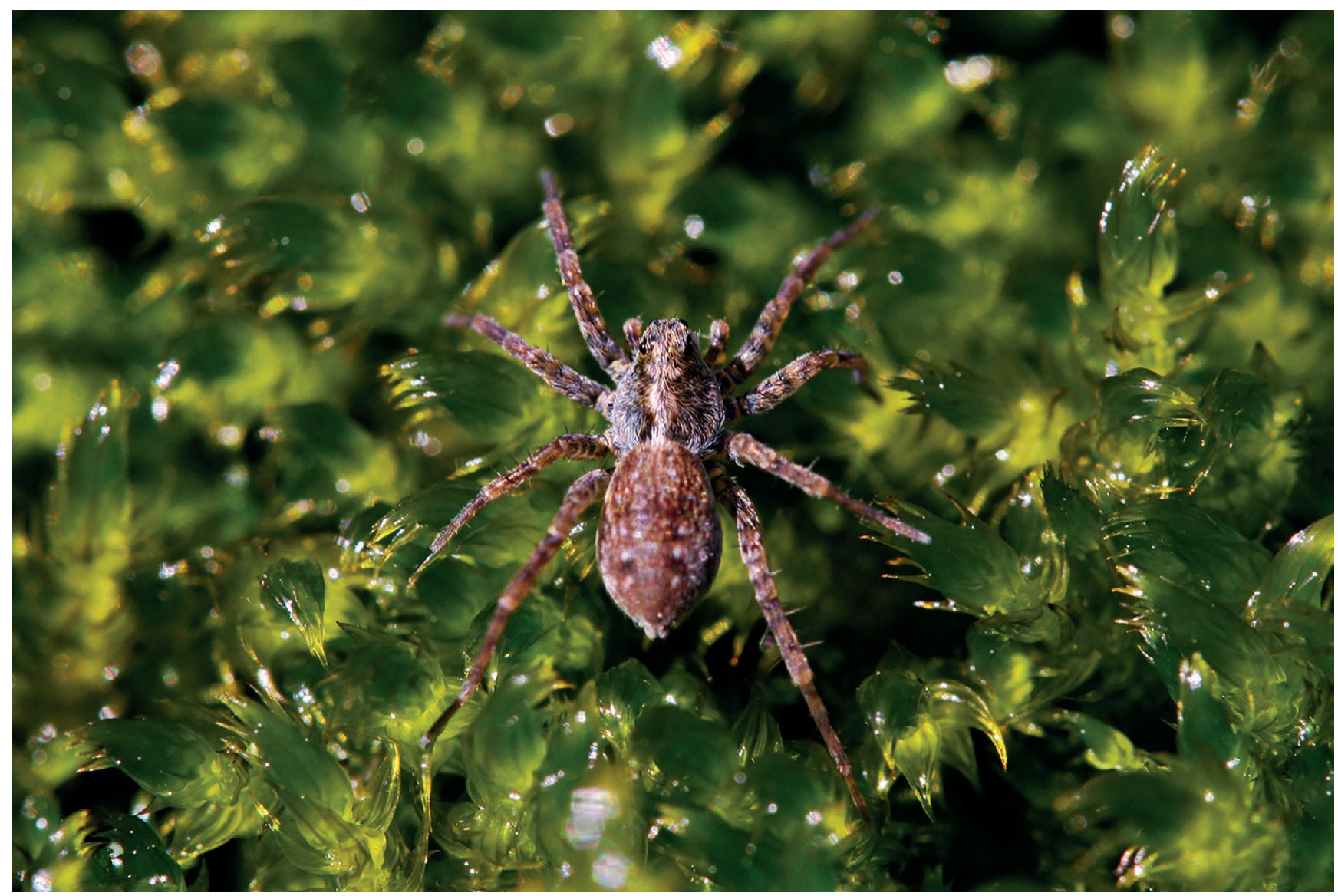

FiG. 8. - Pardosa amentata (Clerck, 1757) (Lycosidae), female. Average body length of the female: 6-7 mm. Courmayeur: Val Ferret. Photograph: Mauro Paschetta 2012.

peat bog, 10.VII.2012, 7 ơ 8 \% , leg. Paschetta, Giuliano, Mammola \& Marguerettaz; same locality, 13.IX.2012, 2 ㅇ, leg. Paschetta, Mammola \& Marguerettaz; Torgnon: Lo Ditor, 1950 m, peat bog, 26.VII-14.VIII.2013, 1 o", leg. Negro; Ayas: Antagnod, 1970$2070 \mathrm{~m}$, alpine pasture, 2007, leg. Franco \& Negro; same locality, ski pistes, 2007, leg. Franco \& Negro.

LiTERATURE. - Isaia 2000, [2005]; Negro et al. 2009, 2010, 2013; [Rossi \& Bosio 2012].

Chorotype. - TUE.

MACROHABITAT. - Alpine prairies, ski pistes, shrublands, wetlands.

-Pardosa monticola (Clerck, 1757)

Literature. - Calloni 1889; Pavesi 1904; di Caporiacco 1928; [Isaia 2005]; [Rossi \& Bosio 2012].

Chorotype. - PAL.

MACROHABITAT. - Not specified.

NOTES. - Old record in need of verification.
- Pardosa morosa (L. Koch, 1870)

LiTERATURE. - Di Caporiacco 1928; [Isaia 2005]; [Rossi \& Bosio 2012].

Chorotype. - TUE.

MacrohabitaT. - Not specified.

NotES. - Old record in need of verification.

Pardosa nigra (C. L. Koch, 1834)

NEW DATA. - Fontainemore, Monte Mars, 1900 m, rocky land, 14.IX.2012, 1 \%, leg. Paschetta \& Mammola.

Literature. - Calloni 1889; Pavesi 1904; di Caporiacco 1928; Isaia 2000, [2005]; Negro et al. 2010, 2013; [Rossi \& Bosio 2012].

Chorotype. - EUR.

MACROHABITAT. - Rocky lands, alpine prairies, ski pistes.

- Pardosa oreophila Simon, 1937

Literature. - Di Caporiacco 1928; [Rossi \& Bosio 2012] 
Chorotype. - SEU.

MACROHABITAT. - Not specified.

Notes. - Old record in need of verification. In the revision of the "saltuaria" group of the genus Pardosa, Wunderlich (1984) reports for the Alps only P. oreophila, while P. saltuaria is known only from Central Europe to Kazakistan. Accordingly, all previous records referring to P. saltuaria (di Caporiacco 1928; Rossi \& Bosio 2012) are here assigned to P. oreophila. See also Muster \& Berendonk (2006).

\section{'Pardosa paludicola (Clerck, 1757)}

LiTERATURE. - Calloni 1889; [Isaia 2005].

Chorotype. - PAL.

MACROHABITAT. - Not specified.

Notes. - According to Tongiorgi (1966: 296) and Pantini \& Isaia (2015), the presence of this species in Italy is not reliable, being easily confused with other species of Pardosa. The occurrence of the species in Aosta Valley is also considered uncertain by Rossi \& Bosio (2012).

\section{Pardosa palustris (Linnaeus, 1758)}

NEW DATA. - Courmayeur: Val Ferret, $1740 \mathrm{~m}$, gravel bed of a river, 14-28.VIII.2013, 1 ơ, leg. Negro; Ayas: Antagnod, 1970-2070 m, alpine pasture, 2007, leg. Franco \& Negro; same locality, ski pistes, 2007, leg. Franco \& Negro; Ayas: Champoluc, 1970-2070 m, ski pistes, 2007, leg. Franco \& Negro.

LiTERATURE. - Di Caporiacco 1928; Tongiorgi 1966; [Isaia 2005]; De Angelis \& Fantoni 2008; Negro et al. 2009; [Rossi \& Bosio 2012].

Chorotype. - OLA.

MaCrohabitAT. - Alpine pastures, ski pistes, gravel beds.

\section{Pardosa prativaga (L. Koch, 1870)}

NEW DATA. - Verrayes: stagno di Loson, 1520 m, grove of reeds/ peat bog, 13.VI-4.VII.2011, $790^{7} 40$ \%, leg. Paschetta, Giuliano; same locality, 4-28.VII.2011, 15 ơ 36 ㅇ, leg. Paschetta, Giuliano; same locality, 28.VII-17.VIII.2011, 5 o' 59 \%, leg. Paschetta, Giuliano; same locality, 18.VIII-15.IX.2011, 29\%, leg. Paschetta, Giuliano; Challand-Saint-Victor: Natural Reserve of Lago di Villa, $820 \mathrm{~m}$, grove of reeds, 13.VI-4.VII.2011, 1 \%, leg. Paschetta $\&$ Giuliano; same locality, wet meadow, 28.VII-17.VIII.2011, 1 \% , leg. Paschetta \& Giuliano; same locality, 18.VIII-15.IX.2011, 1 \%, leg. Paschetta \& Giuliano; Arvier: Lago di Lolair, 1175 m, wet meadow, 14.VI-5.VII.2011, $90^{7} 64$ \% , leg. Paschetta \& Giuliano; same locality, 5-29.VII.2011, 9 o $^{71} 11$, leg. Paschetta \& Giuliano; same locality, 29.VII-18.VIII.2011, 1 o $^{7} 81$, leg. Paschetta \& Giuliano; same locality, 18.VIII-16.IX.2011, 35 \% , leg. Paschetta \& Giuliano; Pont-Saint-Martin: Natural Reserve of Stagno di Holay, 775 m, wet meadow, 5.IV.2012, 3 o", leg. Paschetta \& Isaia; same locality, riparian vegetation, 13.VI4.VII.2011, 1 o, leg. Paschetta \& Giuliano (MRSN-VDA); La Thuile: Comba Thuilette, $2000 \mathrm{~m}$, wet meadow, 16.VIII.2011, 2 ㅇ, leg. Paschetta \& Giuliano.

Literature. - Rossi \& Arnò 1995; [Isaia 2005]; [Rossi \& Bosio 2012].
CHOrotype. - SIE.

MacrohabitaT. - Wetlands, riparian habitats, shrublands.

\section{Pardosa proxima (C. L. Koch, 1847)}

NeW DATA. - La Thuile: Comba Thuilette, 2000 m, wet meadow, 17.VIII.2011, 1 ㅇ, leg. Paschetta \& Giuliano; Pont-Saint-Martin: Natural Reserve of Stagno di Holay, 775 m, wet meadow, 28.VII-17. VIII.2011, 4 ㅇ, leg. Paschetta \& Giuliano; same locality, 18.VIII15.IX.2011, 1 o $^{\Uparrow} 1$ \% , leg. Paschetta \& Giuliano.

Literature. — Di Caporiacco 1928; [Isaia 2005]; [Rossi \& Bosio 2012].

Chorotype. - SIE.

MACROHABITAT. - Wetlands.

\section{-Pardosa pullata (Clerck, 1757)}

LiteraTure. — Calloni 1889; [Isaia 2005]; [Rossi \& Bosio 2012].

Chorotype. - SIE.

MacrohabitAT. - Not specified.

NOTES. - Old record in need of verification.

\section{Pardosa riparia (C. L. Koch, 1833)}

NEW DATA. — Torgnon: Lo Ditor, 1950 m, peat bog, 11-26.VI.2013, $10^{7} 1$ \%, leg. Negro (MRSN-VDA); same locality, 11-26.VI.2013, 5 ơ 2 \%, leg. Negro; same locality, 26.VI-12.VII.2013, 39 ơ 20 \%, leg. Negro; same locality, 12-26.VII.2013, 58 ơ 17 \%, leg. Negro; Torgnon: Lo Ditor, 1950 m, peat bog, 26.VII-14.VIII.2013, 67 o" 31 , leg. Negro; Courmayeur: Val Ferret, 1700 m, grove of reeds, 11.VII.2012, 1 ơ $^{7}$ leg. Paschetta, Giuliano \& Mammola; same locality, gravel bed of a river, 14-28.VIII.2013, 1 \%, leg. Negro; same locality, $1630 \mathrm{~m}$, shrubs, 26.VI-12.VII.2013, $21 \mathrm{o}^{7} 4$ o , leg. Negro; same locality, 26.VII-14.VIII.2013, $40^{7} 9$ ㅇ, leg. Negro; same locality, 14-28.VIII.2013, 4 o , leg. Negro; Challand-Saint-Victor: Natural Reserve of Lago di Villa, 820 m, broadleaved forest, 26.VI12.VII.2013, 1 \%, leg. Negro; Ayas: Antagnod, 1970-2070 m, alpine pasture, 2007, leg. Franco \& Negro; same locality, ski pistes, 2007, leg. Franco \& Negro; Ayas: Champoluc, 1650-1850 m, ski pistes, 2007, leg. Franco \& Negro.

LiTERATURE. — Tongiorgi 1966; [Isaia 2005]; De Angelis \& Fantoni 2008; Negro et al. 2009; [Rossi \& Bosio 2012].

Chorotype. - PAL.

MACROHABITAT. - Wetlands, shrublands, riparian habitats, gravel beds, broadleaved forests, coniferous forests, ski pistes, alpine pastures.

\section{Pardosa saturatior Simon, 1937}

Literature. — Gobbi et al. 2010; [Rossi \& Bosio 2012].

Chorotype. - SEU.

MACROHABITAT. - Rocky lands. 
Pardosa schenkeli Lessert, 1904

Literature. — Isaia 2005; [Rossi \& Bosio 2012].

Chorotype. - SIE.

MACROHABITAT. - Not specified.

\section{Pardosa torrentum Simon, 1876}

NEW DATA. - Courmayeur: Val Ferret, 1700 m, gravel bed of a river, 11.VII.2012, 1 o' 2 ㅇ, leg. Paschetta, Giuliano \& Mammola.

Literature. - Di Caporiacco 1928; [Isaia 2005]; [Rossi \& Bosio 2012].

Chorotype. - SEU.

Macrohabitat. - Gravel beds.

\section{*Pardosa vlijmi Den Hollander \& Dijkstra, 1974}

NEW DATA. - Pont-Saint-Martin: Natural Reserve of Stagno di Holay, 775 m, wet meadow, 4-28.VII.2011, 1 o $^{7} 6$ \% , leg. Paschetta \& Giuliano; same locality, 28.VII-17.VIII.2011, 1 \%, leg. Paschetta \& Giuliano.

CHOROTYPe. - MED.

MACrOHABITAT. - Wetlands.

Notes. - New record for Aosta Valley. P. vlijmi has been described on the base of the courtship behavior. The observation of courting males is currently the only way to identify the species. Recent studies (Chiarle et al., pers. comm.) support the presence of peculiar morphological features, which are useful to distinguish it from the related species of the proxima-group.

\section{Pardosa wagleri (Hahn, 1822)}

Literature. - Isaia 2005; [Rossi \& Bosio 2012].

Chorotype. - PAL.

MACROHABITAT. - Riparian habitats, synanthropic habitats.

\section{*Pirata piraticus (Clerck, 1757)}

NEW DATA. - Brissogne, Saint-Marcel, Quart, Nus: Natural Reserve of Les Îles, $530 \mathrm{~m}$, riparian vegetation, 5-29.VII.2011, 2 \%, leg. Paschetta \& Giuliano; same locality, 18.VIII-16.IX.2011, 3 ơ $^{\prime \prime}$ 10 \%, leg. Paschetta \& Giuliano; Verrayes: stagno di Loson, 1520 m, grove of reeds/peat bog, 13.VI-4.VII.2011, $200^{7} 2$ ㅇ, leg. Paschetta $\&$ Giuliano; same locality, 4-28.VII.2011, 23 ơ 10 \%, leg. Paschetta \& Giuliano; same locality, 28.VII-17.VIII.2011, 10 o' 10 옹 leg. Paschetta \& Giuliano; same locality, 18.VIII-15.IX.2011, 29 o" 44 \% leg. Paschetta \& Giuliano; Courmayeur: Val Ferret, 1700 m, grove of reeds, 11.VII.2011, 1 o $^{7} 1$ \%, leg. Paschetta, Giuliano \& Mammola; Morgex-La Salle: Natural Reserve of Marais, 900 m, grove of reeds, 14.VI-5.VII.2011, 1 \%, leg. Paschetta \& Giuliano; Arvier: Lago di Lolair, 1175 m, wet meadow, 14.VI-5.VII.2011, 12 o $^{7} 10$ \% , leg. Paschetta \& Giuliano; Pont-Saint-Martin: Natural Reserve of
Stagno di Holay, 775 m, riparian vegetation, 13.VIII.2012, 1 은 leg. Paschetta.

Chorotype. - OLA.

MACROHABITAT. - Riparian habitats, wetlands.

Notes. - New record for Aosta Valley.

\section{*Pirata tenuitarsis Simon, 1876}

NEW DATA. - Challand-Saint-Victor: Natural Reserve of Lago di Villa, $820 \mathrm{~m}$, grove of reeds, 13.VI-4.VII.2011, 1 \%, leg. Paschetta \& Giuliano; same locality, 4-28.VII.2011, $20^{7} 3$ ㅇ, leg. Paschetta \& Giuliano; same locality, 28.VII-17.VIII.2011, 3 0̛ 6 \%, leg. Paschetta \& Giuliano; same locality, 18.VIII-15.IX.2011, 1 \&, leg. Paschetta \& Giuliano; same locality, riparian vegetation, 13.VI-4.VII.2011, 1 우, leg. Paschetta \& Giuliano; same locality, 28.VII-17.VIII.2011, $10^{\pi} 2$ ㅇ, leg. Paschetta \& Giuliano; same locality, wet meadow, 13.VI-4.VII.2011, $20^{\star}$, leg. Paschetta \& Giuliano (MNHN); PontSaint-Martin: Natural Reserve of Stagno di Holay, 775 m, riparian vegetation, 28.VII-17.VIII.2011, 1 o", leg. Paschetta \& Giuliano; same locality, 13.VI-4.VII.2011, 1 o", leg. Paschetta \& Giuliano, same locality, 13.VI-4.VII.2011, 2 o", leg. Paschetta \& Giuliano, MRSN-VDA.

Chorotype. - ASE.

MACROHABITAT. - Wetlands, riparian habitats.

NOTES. - New record for Aosta Valley.

\section{*Piratula hygrophila (Thorell, 1872)}

NEW DATA. - Pont-Saint-Martin: Natural Reserve of Stagno di Holay, $775 \mathrm{~m}$, riparian vegetation, 4-28.VII.2011, 1 \%, leg. Paschetta \& Giuliano (MRSN-VDA); same locality, 28.VII-17.VIII.2011, 5 क leg. Paschetta \& Giuliano; same locality, 18.VIII-15.IX.2011, 1 \%, leg. Paschetta \& Giuliano.

Chorotype. - PAL.

MACROHABITAT. - Riparian habitats.

Notes. - New record for Aosta Valley.

Piratula latitans (Blackwall, 1841)

NEW DATA. - Morgex-La Salle: Natural Reserve of Marais, 900 m, riparian forest, 14.VI-5.VII.2011, $90^{7}$, leg. Paschetta \& Giuliano; same locality, 5-29.VII.2011, 1 o'1 1 , leg. Paschetta \& Giuliano; same locality, 29.VII-18.VIII.2011, 2 \% , leg. Paschetta \& Giuliano; same locality, 18.VIII-16.IX.2011, 2 \%, leg. Paschetta \& Giuliano; same locality, grove of reeds, 11-26.VI.2013, 3 o" 1 \%, leg. Negro; same locality, 26.VI-12.VII.2013, 2 \%, leg. Negro; same locality, 12-26.VII.2013, 1 \%, leg. Negro; Challand-Saint-Victor: Natural Reserve of Lago di Villa, 820 m, grove of reeds, 13.VI-4.VII.2011, 1 , leg. Paschetta \& Giuliano (MRSN-VDA); same locality, 13.VI4.VII.2011, 6 ơ $^{7}$ ㅇ, leg. Paschetta \& Giuliano; same locality, wet meadow, 13.VI-4.VII.2011, 3 o", leg. Paschetta \& Giuliano; same locality, grove of reeds, 4-28.VII.2011, $1 \sigma^{7} 1$ \% , leg. Paschetta \& Giuliano; same locality, 28.VII-17.VIII.2011, 2 o' 9 \%, leg. Paschetta \& Giuliano; same locality, 18.VIII-15.IX.2011, 1 ơ $^{7}$ leg. Paschetta \& Giuliano; Arvier: Lago di Lolair, 1175 m, wet meadow, 14.VI-5. VII.2011, 7 ơ 1 \%, leg. Paschetta \& Giuliano; same locality, 5-29. VII.2011, $1 \sigma^{\prime \prime}$, leg. Paschetta \& Giuliano; same locality, 29.VII- 
18.VIII.2011, 4 \%, leg. Paschetta \& Giuliano; Verrayes: stagno di Loson, $1520 \mathrm{~m}$, grove of reeds/peat bog, 4-28.VII.2011, $1 \mathrm{o}^{7}$, leg. Paschetta, Giuliano.

LiterATURE. - Rossi \& Arnò 1995; [Isaia 2005]; [Rossi \& Bosio 2012].

Chorotype. - EUR.

MACROHABITAT. - Riparian habitats, wetlands.

\section{*Trochosa hispanica Simon, 1870}

NEW DATA. - Brissogne, Saint-Marcel, Quart, Nus: Natural Reserve of Les Îles, $526 \mathrm{~m}$, riparian forest, 11-26.VI.2013, 10 ơ 2 을 leg. Negro; same locality, 28.VIII-16.IX.2013, 2 ơ, leg. Negro; same locality, 28.VIII-16.IX.2013, 1 ơ, leg. Negro; Challand-Saint-Victor: Natural Reserve of Lago di Villa, $820 \mathrm{~m}$, wet meadow, 13.VI-4. VII.2011, 1 ơ $^{7}$ \% , leg. Paschetta \& Giuliano; same locality, 4-28. VII.2011, 3 ơ 1 \%, leg. Paschetta \& Giuliano; same locality, grove of reeds, 13.VI-4.VII.2011, 4 0", leg. Paschetta \& Giuliano; same locality, 4-28.VII.2011, 2 \% , leg. Paschetta \& Giuliano; same locality, 28.VII-17.VIII.2011, 2 \% leg. Paschetta \& Giuliano; same locality, 18.VIII-15.IX.2011, 1 \%, leg. Paschetta \& Giuliano; same locality, broadleaved forest, 11-26.VI.2013, 3 o", leg. Negro; same locality, meadow, 11-26.VI.2013, 1 o', leg. Negro; Saint Christophe: Tsatelet, $750 \mathrm{~m}$, broadleaved forest, 24.V-13.VI.2012, $3 \mathrm{o}^{7}$, leg. Negro; Pont-Saint-Martin: Natural Reserve of Stagno di Holay, $702 \mathrm{~m}$, broadleaved forest (Castanea sativa), 11-26.VI.2013, $11 \mathrm{O}^{7}$, leg. Negro; same locality, 26.VI-12.VII.2013, 5 o", leg. Negro; same locality, 28.VIII-16.IX.2013, $20^{\text {" }}$, leg. Negro; same locality, alpine grassland, 11-26.VI.2013, $30^{\text {7 }}$, leg. Negro; same locality, 26.VI-12.VII.2013, 60 " leg. Negro; Gressan: Natural Reserve of Côte de Gargantua, 700 m, broadleaved forest, 3-24.V.2012, 3 ㅇ, leg. Negro; same locality, 24.V-13.VI.2012, 1 ơ $^{\prime \prime}$ leg. Negro; same locality, 13.VI-4.VII.2012, 2 o', leg. Negro; same locality, 4-25. VII.2012, 1 \& , leg. Negro; Sarre-Saint Pierre: Monte Torretta, $900 \mathrm{~m}$, broadleaved forest, 24.V-13.VI.2012, 1 o $^{*}$, leg. Negro; same locality, dry meadow, 13.VI-4.VII.2012, $20^{7}$, leg. Negro; Arvier: Lago di Lolair, 1175 m, wet meadow, 5-29.VII.2011, 1 o", leg. Paschetta \& Giuliano.

Chorotype. - TUM.

MACROHABITAT. - Riparian habitats, wetlands, broadleaved forests, alpine grasslands, shrublands.

Notes. - New record for Aosta Valley.

\section{Trochosa ruricola (De Geer, 1778)}

NEW DATA. - Morgex-La Salle: Natural Reserve of Marais, 900 m, riparian forest, 14.VI-5.VII.2011, 3 \% , leg. Paschetta \& Giuliano; same locality, 5-29.VII.2011, 1 \&, leg. Paschetta \& Giuliano; same locality, 18.VIII-16.IX.2011, 1 \% , leg. Paschetta \& Giuliano; same locality, wet meadow, 29.VII-18.VIII.2011, 1 \%, leg. Paschetta \& Giuliano; same locality, 18.VIII-16.IX.2011, $10^{3} 1$ 웅 leg. Paschetta \& Giuliano; Verrayes: stagno di Loson, 1520 m, grove of

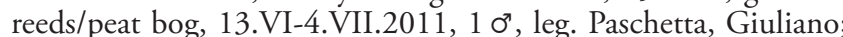
same locality, 18.VIII-15.IX.2011, 1 o", leg. Paschetta \& Giuliano; Arvier: Lago di Lolair, 1175 m, wet meadow, 14.VI-5.VII.2011, 3 o", leg. Paschetta \& Giuliano; same locality, 5-29.VII.2011, 3 o", leg. Paschetta \& Giuliano; same locality, 29.VII-18.VIII.2011, 10 o", leg. Paschetta \& Giuliano; same locality, 18.VIII-16.IX.2011, $1 \sigma^{\prime \prime}$, leg. Paschetta \& Giuliano; Saint Christophe: Tsatelet, 750 m, broadleaved forest, 13.VI-4.VII.2012, 1 ơ, leg. Negro; Sarre-Saint
Pierre: Monte Torretta, 900 m, dry meadow, 13.VI-4.VII.2012, 1 ㅇ, leg. Negro.

Literature. - Pavesi 1904; Rossi \& Arnò 1995; [Isaia 2005]; De Angelis \& Fantoni 2008; Negro et al. 2009; [Rossi \& Bosio 2012].

Chorotype. - OLA.

MACROHABITAT. - Riparian habitats, wetlands, shrublands, broadleaved forests.

\section{Trochosa terricola Thorell, 1856}

NEW DATA. — Ayas: Antagnod, 1970-2070 m, coniferous forests, 2007, leg. Franco \& Negro.

LiTERATURE. — Calloni 1889; [Isaia 2005]; De Angelis \& Fantoni 2008; [Rossi \& Bosio 2012].

Chorotype. - OLA.

MACROHABITAT. - Coniferous forests.

\section{Xerolycosa miniata (C. L. Koch, 1834)}

NEW DATA. - Challand-Saint-Victor: Natural Reserve of Lago di Villa, 820 m, wet meadow, 28.VII-17.VIII.2011, 1 \%, leg. Paschetta \& Giuliano; Pont-Saint-Martin: Natural Reserve of Stagno di Holay, 775 m, wet meadow, 4-28.VII.2011, 1 o', leg. Paschetta \& Giuliano (MRSN-VDA); Brissogne, Saint-Marcel, Quart, Nus: Natural Reserve of Les Îles, 530 m, dry meadow, 14.VI-5.VII.2011, $10^{\prime \prime}$, leg. Paschetta \& Giuliano.

Literature. — De Angelis \& Fantoni 2008; [Rossi \& Bosio 2012].

Chorotype. - PAL.

Macrohabitat. - Alpine pastures, wetlands, shrublands.

\section{Xerolycosa nemoralis (Westring, 1861)}

NeW DATA. - Aymavilles: Pont d'Ael, $800 \mathrm{~m}$, broadleaved forest, 24.V-13.VI.2012, 1 ㅇ, leg. Negro; same locality, 13.VI-4.VII.2012, 2 o 1 ơ leg. Negro; Saint Denis: Brison-Cly, 1000 m, dry meadow, 24.V-13.VI.2012, $10^{\top 1} 1$ \%, leg. Negro; same locality, 13.VI-4.VII.2012, $10^{7} 3$ \% , leg. Negro; same locality, 4-25.VII.2012, $10^{7} 1$ ㅇ, leg. Negro (MRSN-VDA); same locality, 4-25.VII.2012, 3 o', leg. Negro; Pont- Saint-Martin: Natural Reserve of Stagno di Holay, 616 m, alpine grassland, 11-26.VI.2013, 10 " leg. Negro; same locality, 12-26.VII.2013, 1 o", leg. Negro; Arvier: Lago di Lolair, 1175 m, wet meadow, 5-29.VII.2011, 1 ơ, leg. Paschetta \& Giuliano; same locality, broadleaved forest, 3-24.V.2012, 1 ㅇ, leg. Negro; Ayas: Champoluc, 1650-1850 m, ski pistes, 2007, leg. Franco \& Negro.

Literature. - Isaia 2000, [2005]; De Angelis \& Fantoni 2008; Negro et al. 2009; Gobbi et al. 2010; [Rossi \& Bosio 2012].

Chorotype. - PAL.

MacrohabitaT. - Broadleaved forests, coniferous forests, ski pistes, shrublands, wetlands, alpine grasslands. 
Family MimETiDAE Simon, 1881

${ }^{*}$ Ero aphana (Walckenaer, 1802)

New Data. - Aymavilles: Pont d'Ael, 800 m, dry meadow, 24.V13.VI.2012, 1 o', leg. Negro (MRSN-VDA).

Chorotype. - EUR.

Macrohabitat. - Shrublands.

Notes. - New record for Aosta Valley.

\section{*Ero furcata (Villers, 1789)}

NEW DATA. - Brissogne, Saint-Marcel, Quart, Nus: Natural Reserve of Les Îles, $530 \mathrm{~m}$, riparian vegetation, 14.VI-5.VII.2011, 1 \%, leg. Paschetta \& Giuliano.

Chorotype. - PAL.

MACROHABITAT. - Riparian habitats.

Notes. - New record for Aosta Valley.

Family MitUrgidAe Simon, 1886

•Zora manicata Simon, 1878

Literature. - Simon 1878; Pavesi 1904; [Isaia 2005]; [Rossi \& Bosio 2012].

Chorotype. - EUM.

MACROHABITAT. - Not specified.

NOTES. - Old record in need of verification.

Zora silvestris Kulczyński, 1897

Literature. - De Angelis \& Fantoni 2008; [Rossi \& Bosio 2012].

Chorotype. - CAE.

Macrohabitat. - Not specified.

Zora spinimana (Sundevall, 1833)

NEW DATA. - Morgex-La Salle: Natural Reserve of Marais, 900 m, riparian forest, 14.VI-5.VII.2011, 1 o", leg. Paschetta \& Giuliano; same locality, wet meadow, 5-29.VII.2011, 1 \%, leg. Paschetta \& Giuliano; same locality, grove of reeds, 12-26.VII.2013, 1 o", leg. Negro; same locality, riparian forest, 11-26.VI.2013, $20^{7}$, leg. Negro; Challand-Saint-Victor: Natural Reserve of Lago di Villa, $820 \mathrm{~m}$, broadleaved forest, 11-26.VI.2013, $20^{\text {* }}$, leg. Negro; same locality, 26.VI-12.VII.2013, 3 ơ, leg. Negro; same locality, meadow, 26.VI-12.VII.2013, 1 ơ, leg. Negro; Saint Christophe: Tsatelet, $750 \mathrm{~m}$, broadleaved forest, 24-13.VI.2012, 1 \%, leg. Negro; same locality, 4-25.VII.2012, 1 \%, leg. Negro (MRSN-VDA); Arvier: Lago di Lolair, 1175 m, wet meadow, 18.VIII-16.IX.2011, 1 \%, leg. Paschetta \& Giuliano; Ayas: Antagnod, 1970-2070 m, alpine pasture, leg. Franco \& Negro.
Literature. — Negro et al. 2009; [Rossi \& Bosio 2012].

Chorotype. - PAL.

MACROHABITAT. - Riparian habitats, broadleaved forests, alpine grasslands, alpine pastures, wetlands.

Family NestiCidAe Simon, 1894

Nesticus cellulanus (Clerck, 1757)

LiterATURE. - Arnò \& Lana 2005; Isaia et al. 2011; [Rossi \& Bosio 2012].

Chorotype. - OLA.

MACROHABITAT. - Caves.

Nesticus eremita Simon, 1879

LiterATURE. - Isaia et al. 2011; [Rossi \& Bosio 2012].

Chorotype. - SEU.

MaCrohabitat. - Caves.

Family OECOBIIDAE Blackwall, 1862

Oecobius cellariorum (Dugès, 1836)

Literature. - Rossi \& Bosio 2012.

Chorotype. - COS.

MACROHABITAT. - Broadleaved forests.

Family OXYOPIDAE Thorell, 1870

Oxyopes ramosus (Martini \& Goeze, 1778)

LITERATURE. - Isaia 2005; [Rossi \& Bosio 2012].

CHOROTYPe. - SIE.

MacrohabitAT. - Not specified.

Family PHILODROMIDAe Thorell, 1870

Philodromus aureolus (Clerck, 1757)

NEW DATA. - Challand-Saint-Victor: Natural Reserve of Lago di Villa, $820 \mathrm{~m}$, broadleaved forest, 26.VI-12.VII.2013, 1 \%, leg. Negro; La Thuile: Comba Thuilette, 2000 m, rocky land, 17.VIII.2011, 1 \&, leg. Paschetta \& Giuliano.

Literature. - Calloni 1889; Rossi \& Arnò 1995; [Isaia 2005]; [Rossi \& Bosio 2012]. 
Chorotype. - PAL.

MACROHABITAT. - Riparian habitats, broadleaved forests, rocky land.

Philodromus cespitum (Walckenaer, 1802)

LiterATURE. - Rossi \& Arnò 1995; [Isaia 2005]; De Angelis \& Fantoni 2008; Negro et al. 2009; [Rossi \& Bosio 2012].

Chorotype. - OLA.

MACROHABITAT. - Broadleaved forests, shrublands.

Philodromus collinus C. L. Koch, 1835

Literature. - De Angelis \& Fantoni 2008; Negro et al. 2009; [Rossi \& Bosio 2012].

CHorotype. - EUR

MACrohabitaT. - Coniferous forests.

*Philodromus emarginatus (Schrank, 1803)

NEW DATA. — Courmayeur: Val Ferret, 1700 m, peat bog, 11.VII.2012, 1 \%, leg. Paschetta, Giuliano \& Mammola.

Chorotype. - PAL.

Macrohabitat. - Wetlands.

Notes. - New record for Aosta Valley.

•Philodromus fuscolimbatus Lucas, 1846

Literature. - Di Caporiacco 1928; [Isaia 2005]; [Rossi \& Bosio 2012].

Chorotype. - MED.

MACROHABITAT. - Not specified.

NOTES. - Old record in need of verification.

•Philodromus laricium Simon, 1875

Literature. - Pavesi 1904; [Isaia 2005]; [Rossi \& Bosio 2012].

Chorotype. - SEU.

MACROHABITAT. - Not specified.

NOTES. - Old record in need of verification.

Philodromus margaritatus (Clerck, 1757)

Literature. - Pavesi 1904; [Isaia 2005]; De Angelis \& Fantoni 2008; [Rossi \& Bosio 2012].

Chorotype. - PAL.

MacrohabitaT. - Not specified.
•Philodromus poecilus (Thorell, 1872)

Literature. — Di Caporiacco 1928; [Isaia 2005]; [Rossi \& Bosio 2012].

Chorotype. - PAL.

MacrohabitaT. - Not specified.

NOTES. - Old record in need of verification.

Philodromus praedatus O. P.-Cambridge, 1871

Literature. - Isaia 2000; [Isaia 2005]; [Rossi \& Bosio 2012].

CHOROTYPe. - SIE.

MacrohabitaT. - Alpine prairies.

\section{Philodromus vagulus Simon, 1875}

NEW DATA. - Ayas: Antagnod, 1970-2070 m, alpine pasture, 2007, leg. Franco \& Negro; La Thuile: Comba Thuilette, $2000 \mathrm{~m}$, rocky land, 10.VII.2012, 2 \%, leg. Paschetta \& Giuliano Mammola, Marguerettaz.

LiteraTuRE. - Di Caporiacco 1928; [Isaia 2005]; De Angelis \& Fantoni 2008; Negro et al. 2009; [Rossi \& Bosio 2012].

Chorotype. - EUR.

MACROHABITAT. - Synanthropic habitats, coniferous forests, alpine pastures, rocky lands.

-Thanatus arenarius L. Koch, 1872

Literature. — Di Caporiacco 1928; [Isaia 2005]; [Rossi \& Bosio 2012].

Chorotype. - PAL.

MacrohabitaT. - Not specified.

NotEs. - Old record in need of verification.

\section{Thanatus atratus Simon, 1875}

NEW DATA. - Pont-Saint-Martin: Natural Reserve of Stagno di Holay, 775 m, wet meadow, 13.VI-4.VII.2011, 1 o', leg. Paschetta \& Giuliano, (MRSN-VDA); Brissogne, Saint-Marcel, Quart, Nus: Natural Reserve of Les Îles, 530 m, dry meadow, 5-29.VII.2011, 2 \%, leg. Paschetta \& Giuliano; same locality, 29.VII-18.VIII.2011, 2 \%, leg. Paschetta \& Giuliano.

Literature. — Di Caporiacco 1928; [Isaia 2005]; [Rossi \& Bosio 2012].

Chorotype. - PAL.

MacrohabitAT. — Wetlands, shrublands. 
Thanatus coloradensis Keyserling, 1880

LitERATURE. — Isaia 2005; [Rossi \& Bosio 2012].

Chorotype. - OLA.

Macrohabitat. - Not specified.

\section{Thanatus formicinus (Clerck, 1757)}

Literature. - Negro et al. 2009.

Chorotype. - OLA.

MACROHABiTAT. - Alpine prairies.

NotEs. - The occurrence of this species in Aosta Valley was considered doubtful by Rossi \& Bosio (2012).

-Thanatus oblongiusculus (Lucas, 1846)

LiterATURE. - Di Caporiacco 1928; [Isaia 2005]; [Rossi \& Bosio 2012].

Chorotype. - SIE.

MACROHABITAT. - Not specified.

NOTES. - Old record in need of verification.

•Thanatus striatus C. L. Koch, 1845

Literature. — Rossi \& Bosio 2012.

Chorotype. - OLA.

MACROHABITAT. - Riparian habitats.

Notes. - See Tegenaria domestica.

Tibellus oblongus (Walckenaer, 1802)

Literature. — Rossi \& Arnò 1995; [Isaia 2005]; Negro et al. 2009; [Rossi \& Bosio 2012].

Chorotype. - OLA.

MACROHABITAT. - Shrublands, broadleaved forests.

Family Pholcidae C. L. Koch, 1850

Pholcus phalangioides (Fuesslin, 1775)

Literature. - Isaia 2005; [Rossi \& Bosio 2012].

Chorotype. - COS.

MACROHABITAT. - Synanthropic habitats.
Family PHrUROLITHIDAE Banks, 1892

Phrurolithus festivus (C. L. Koch, 1835)

NEW DATA. - Challand-Saint-Victor: Natural Reserve of Lago di Villa, 820 m, wet meadow, 13.VI-4.VII.2011, 1 \%, leg. Paschetta \& Giuliano; Brissogne, Saint-Marcel, Quart, Nus: Natural Reserve of Les Îles, 530 m, dry meadow,14.VI-5.VII.2011, 3 o' 2 \%, leg. Paschetta \& Giuliano; same locality, 5-29.VII.2011, 2 ơ 9 웅, leg. Paschetta \& Giuliano; same locality, 29.VII-18.VIII.2011, 15 $q$, leg. Paschetta \& Giuliano; same locality, 18.VIII-16.IX.2011, 2 \%, leg. Paschetta \& Giuliano; same locality, riparian forest, 26.VI-12. VII.2013, 1 ơ, leg. Negro; Challand-Saint-Victor: Natural Reserve of Lago di Villa, $820 \mathrm{~m}$, wet meadow, 13.VI-4.VII.2011, $1 \mathrm{o}^{7}$, leg. Paschetta \& Giuliano; same locality, broadleaved forest, 1226.VII.2013, $10^{\text {T }} 3$ \% , leg. Negro; Arvier: Lago di Lolair, 1175 m, wet meadow, 14.VI-5.VII.2011, $10^{7}$, leg. Paschetta \& Giuliano; Brissogne, Saint-Marcel, Quart, Nus: Natural Reserve of Les Îles, 530 m, dry meadow, 14.VI-5.VII.2011, 1 o $^{7} 1$ ㅇ, leg. Paschetta \& Giuliano; Arvier: Lago di Lolair, 1175 m, wet meadow, 29.VII-18. VIII.2011, 1 \%, leg. Paschetta \& Giuliano; Pont-Saint-Martin: Natural Reserve of Stagno di Holay, $702 \mathrm{~m}$, broadleaved forest (Castanea sativa), 11-26.VI.2013, 1 o', leg. Negro; same locality, wet meadow, 4-28.VII.2011, 1 ơ 1 \%, leg. Paschetta \& Giuliano (MRSN-VDA); same locality, 18.VIII-15.IX.2011, 2 ㅇ, leg. Paschetta \& Giuliano; same locality, broadleaved forest (Castanea sativa), 12-26.VII.2013, 1 ㅇ, leg. Negro; same locality, 26.VI-12.VII.2013, 1 ㅇ, leg. Negro; Arvier: Lago di Lolair, 1175 m, wet meadow, 14.VI-5.VII.2011, $10^{\text {o', }}$ leg. Paschetta \& Giuliano; same locality, 29.VII-18.VIII.2011, 1 ㅇ, leg. Paschetta \& Giuliano; Ayas: Antagnod, 1970-2070 m, alpine pasture, leg. Franco \& Negro.

Literature. - Rossi \& Arnò 1995; [Isaia 2005]; [Rossi \& Bosio 2012].

Chorotype. - PAL.

MACROHABITAT. - Wetlands, shrublands, riparian habitats, broadleaved forests, alpine pastures.

\section{Phrurolithus minimus C. L. Koch, 1839}

NEW DATA. - Challand-Saint-Victor: Natural Reserve of Lago di Villa, 820 m, broadleaved forest, 26.VI-12.VII.2013, 2 o', leg. Negro; Challand-Saint-Victor: Natural Reserve of Lago di Villa, $820 \mathrm{~m}$, grove of reeds, 13.VI-4.VII.2011, 5 o 1 \%, leg. Paschetta \& Giuliano; same locality, wet meadow, 13.VI-4.VII.2011, 2 o", leg. Paschetta \& Giuliano; same locality, broadleaved forest, 26.VI-12.VII.2013, 1 ơ, leg. Negro; same locality, 12-26.VII.2013, 1 o" 1 \%, leg. Negro; same locality, broadleaved forest, 26.VI-12.VII.2013, 3 o" 1 운, leg. Negro; same locality, 26.VII-14.VIII.2013, 1 ơ 3 \%, leg. Negro; same locality, meadow, 11-26.VI.2013, 1 \%, leg. Negro; MorgexLa Salle: Natural Reserve of Marais, 896 m, riparian forest, 11-26. VI.2013, $10^{n}$, leg. Negro; same locality, grove of reeds, 11-26. VI.2013, 2 o" 1 \%, leg. Negro; same locality, 26.VII-14.VIII.2013, 1 o', leg. Negro; same locality, 26.VI-12.VII.2013, 1 ơ", leg. Negro; same locality, riparian forest, 26.VI-12.VII.2013, $3 \sigma^{7}$, leg. Negro; same locality, 12-26.VII.2013, $20^{\text {T } 1} 1$ \%, leg. Negro; same locality,

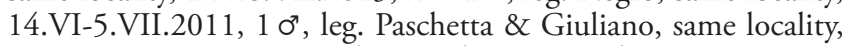
29.VII-18.VIII.2011, 1 ơ, leg. Paschetta \& Giuliano.

Literature. - Isaia 2005; [Rossi \& Bosio 2012].

CHOrotype. - EUR.

MACROHABITAT. - Broadleaved forests, wetlands, alpine grasslands, riparian habitats. 
Family PISAURIDAE Simon, 1890

-Dolomedes fimbriatus (Clerck, 1757)

LiterATURE. — Rossi \& Bosio 2012.

CHOROTYPe. - PAL.

MACrOHABitaT. - Wetlands.

Notes. - See notes on Tegenaria domestica.

Pisaura mirabilis (Clerck, 1757)

(Fig. 9)

NEW DATA. - Saint Denis: Brison-Cly, 1000 m, broadleaved forest, 24.V-13.VI.2012, 1 ơ $^{7}$, leg. Negro; Arvier: Lago di Lolair, 1175 m, broadleaved forest, 24.V-13.VI.2012, 1 ơ, leg. Negro; La Thuile: Comba Thuilette, $2000 \mathrm{~m}$, wet meadow, 9.VII.2012, 1 \% , leg. Paschetta; Pont-Saint-Martin: Natural Reserve of Stagno di Holay, 775 m, riparian vegetation, 13.VIII.2012, 1 \&, leg. Paschetta.

Literature. - Rossi \& Arnò 1995; [Isaia 2005]; Negro et al. 2009; Gobbi et al. 2010; [Rossi \& Bosio 2012].

Chorotype. - ASE.

MAcrohabitaT. - Broadleaved forests, wetlands, riparian habitats, shrublands.

Family SALTICIDAE Blackwall, 1841

\section{Aelurillus v-insignitus (Clerck, 1757)}

NEW DATA. - Pont-Saint-Martin: Natural Reserve of Stagno di Holay, 616 m, alpine grassland, 11-26.VI.2013, 1 o', leg. Negro; same locality, 14-28.VIII.2013, 2 \% , leg. Negro; same locality, 1226.VII.2013, 1 o $^{7} 1$ \%, leg. Negro (MRSN-VDA); Aymavilles: Pont d'Ael, 800 m, dry meadow, 25.VII-20.VIII.2012, 1 우, leg. Negro; Brissogne, Saint-Marcel, Quart, Nus: Natural Reserve of Les Iles, 530 m, dry meadow, 18.VIII-16.IX.2011, 1 ㅇ, leg. Paschetta \& Giuliano; same locality, 26.VI-12.VII.2013, 3 \% , leg. Negro; same locality, 11-26.VI.2013, 1 o", leg. Negro; Ayas: Antagnod, 19702070 m, ski pistes , 2007, leg. Franco \& Negro.

Literature. - Fontana et al. 1996; [Isaia 2005]; Negro et al. 2009; [Rossi \& Bosio 2012].

Chorotype. - PAL.

MACROHABITAT. - Alpine grasslands, shrublands, ski pistes, alpine prairies.

Ballus chalybeius (Walckenaer, 1802)

Literature. - Isaia 2005; [Rossi \& Bosio 2012].

CHOROTyPe. - EUR.

MacrohabitaT. - Not specified.
${ }^{*}$ Chalcoscirtus infimus (Simon, 1868)

NEW DATA. - Brissogne, Saint-Marcel, Quart, Nus: Natural Reserve of Les Îles, 526 m, dry meadow, 11-26.VI.2013, 1 o", leg. Negro (MRSN-VDA).

Chorotype. - TUE.

MACROHABITAT. - Shrublands.

Notes. - New record for Aosta Valley.

\section{Euophrys frontalis (Walckenaer, 1802)}

NEW DATA. — Morgex-La Salle: Natural Reserve of Marais, 896 m, riparian forest, 11-26.VI.2013, 2 \% leg. Negro; Challand-Saint-Victor: Natural Reserve of Lago di Villa, $820 \mathrm{~m}$, broadleaved forest, 26.VI12.VII.2013, 1 \% , leg. Negro; Saint Christophe: Tsatelet, $750 \mathrm{~m}$, broadleaved forest, 13.VI-4.VII.2012, $10^{7} 1 \%$, leg. Negro (MRSNVDA); Sarre-Saint Pierre: Monte Torretta, $900 \mathrm{~m}$, dry meadow, 24.V-13.VI.2012, 1 \%, leg. Negro (MNHN); Pont-Saint-Martin: Natural Reserve of Stagno di Holay, 616 m, alpine grassland, 14-28. VIII.2013, 1 0", leg. Negro; Gressan: Natural Reserve of Côte de Gargantua, 700 m, broadleaved forest, 3-24.V.2012, 1 \% , leg. Negro.

LiTERATURE. — De Angelis \& Fantoni 2008; [Isaia 2005]; [Rossi \& Bosio 2012].

CHOROTYPe. - PAL.

MACROHABITAT. - Riparian habitats, broadleaved forests, shrublands, alpine grasslands.

\section{Evarcha arcuata (Clerck, 1757)}

Literature. - Rossi \& Arnò 1995; [Isaia 2005]; [Rossi \& Bosio 2012].

Chorotype. - PAL.

MACROHABITAT. - Synanthropic habitats, wetlands.

\section{Evarcha falcata (Clerck, 1757)}

NeW DATA. - Aymavilles: Pont d'Ael, 800 m, dry meadow, 25.VII20.VIII.2012, 1 \%, leg. Negro.

Literature. - Rossi \& Bosio 2012.

Chorotype. - PAL.

MACROHABITAT. - Shrublands.

Evarcha laetabunda (C. L. Koch, 1846)

Literature. - Rossi \& Arnò 1995; [Isaia 2005]; [Rossi \& Bosio 2012].

Chorotype. - PAL.

MACROHABITAT. - Shrublands. 


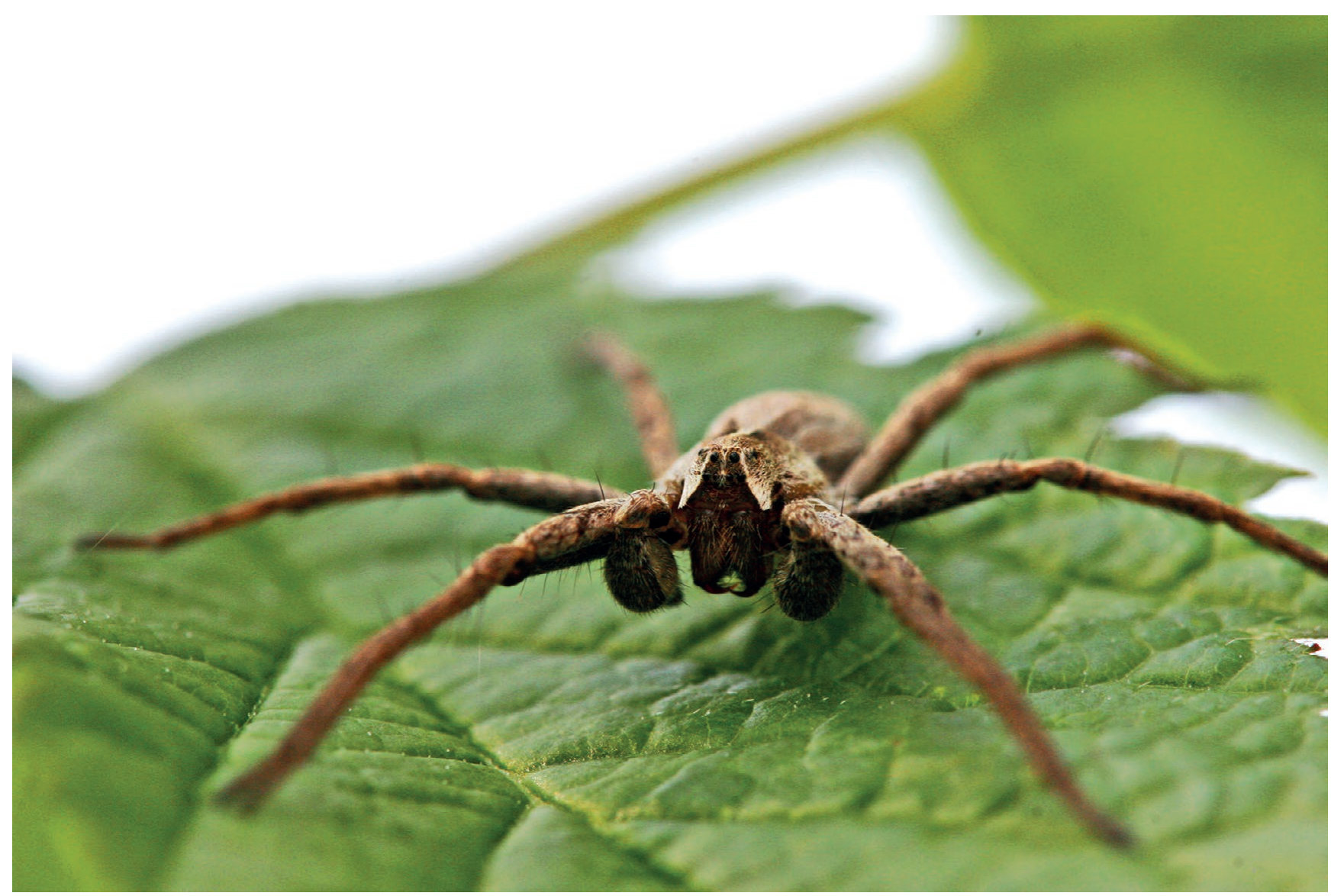

FIG. 9. - Pisaura mirabilis (Clerck, 1757) (Pisauridae), male. Average body length of the male: 10-13 mm. Arvier: Lago di Lolair. Photograph: Mauro Paschetta 2012.

Heliophanus aeneus (Hahn, 1832)

NeW DATA. - La Thuile: Comba Thuilette, $2000 \mathrm{~m}$, rocky land, 13.IX.2012, 2 o", leg. Paschetta, Mammola \& Marguerettaz.

LITERATURE. — Pavesi 1904; Fontana et al. 1996; Isaia 2000, [2005]; [Rossi \& Bosio 2012].

Chorotype. - PAL.

Macrohabitat. - Coniferous forests, rocky lands.

•Heliophanus auratus C. L. Koch, 1835

LiterATURE. - Di Caporiacco 1928; [Isaia 2005]; [Rossi \& Bosio 2012].

Chorotype. - ASE.

MacrohabitaT. - Not specified.

NOTES. - Old record in need of verification.

Heliophanus cupreus (Walckenaer, 1802)

NEW DATA. - Challand-Saint-Victor: Natural Reserve of Lago di Villa, 820 m, wet meadow, 28.VII-17.VIII.2011, 1 \&, leg. Paschetta \&
Giuliano; same locality, 13.VI-4.VII.2011, 1 \% , leg. Paschetta \& Giuliano; Morgex-La Salle: Natural Reserve of Marais, 896 m, riparian forest, 11-26.VI.2013, 2 \% , leg. Negro; same locality, 26.VI12.VII.2013, $10^{7}$, leg. Negro; same locality, grove of reeds, 12-26. VII.2013, 1 ơ 1 \%, leg. Negro; Aymavilles: Pont d'Ael, 800 m, dry meadow, 24.V-13.VI.2012, 1 \%, leg. Negro; Courmayeur: Val Ferret, $1630 \mathrm{~m}$, shrubs, 26.VI-12.VII.2013, 1 o", leg. Negro; same locality, $1700 \mathrm{~m}$, gravel bed of a river, 11.VII.2012, $1 \mathrm{o}^{\circ} 1$ \% , leg. Paschetta, Giuliano \& Mammola; Pont-Saint-Martin: Natural Reserve of Stagno di Holay, 775 m, riparian vegetation, 5.IV.2012, 1 ơ, leg. Paschetta \& Isaia; same locality, 13.VIII.2012, 1 \%, leg. Paschetta; Ayas: Champoluc, 1650-1850 m, ski pistes, 2007, leg. Franco \& Negro.

Literature. - Rossi \& Arnò 1995; Fontana et al. 1996; [Isaia 2005]; Negro et al. 2009; [Rossi \& Bosio 2012].

CHOrotype. - SIE.

MACROHABITAT. - Wetlands, riparian habitats, gravel beds, shrublands, ski pistes, broadleaved forests.

\section{Heliophanus flavipes (Hahn, 1832)}

NEW DATA. - Torgnon: Lo Ditor, 1950 m, peat bog, 12-26. VII.2013, 1 ơ, leg. Negro; Ayas: Antagnod, 1970-2070 m, 2007, leg. Franco \& Negro.

Literature. - Fontana et al. 1996; [Isaia 2005]; [Rossi \& Bosio 2012]. 
Chorotype. - PAL.

MACROHABITAT. - Shrublands, wetlands.

\section{Heliophanus kochii Simon, 1868}

NEW DATA. — Morgex-La Salle: Natural Reserve of Marais, 900 m, wet meadow, 14.VI-5.VII.2011, 2 ㅇ, leg. Paschetta \& Giuliano.

LiTerATURE. - Fontana et al. 1996; [Isaia 2005]; [Rossi \& Bosio 2012].

Chorotype. - EUM.

MACROHABITAT. - Alpine grasslands, wetlands.

\section{Heliophanus lineiventris Simon, 1868}

NEW DATA. - Fontainemore, Monte Mars, 1900 m, rocky land, 17.IX.2012, 1 ơ, leg. Paschetta \& Marguerettaz; Brissogne, SaintMarcel, Quart, Nus: Natural Reserve of Les Iles, 530 m, grove of reeds, 17.VIII.2011, 1 \% , leg. Paschetta \& Giuliano.

LiterATURE. - Fontana et al. 1996; [Isaia 2005]; [Rossi \& Bosio 2012].

Chorotype. - PAL.

MacrohabitaT. - Rocky lands, wetlands.

\section{Heliophanus tribulosus Simon, 1868}

NEW DATA. - Sarre-Saint Pierre: Monte Torretta, 900 m, dry meadow, 3-24.V.2012, 1 o", leg. Negro.

Literature. — Rossi \& Bosio 2012.

CHOROTYPe. - SIE.

MaCrohabitaT. - Shrublands, synanthropic habitats.

\section{*Marpissa nivoyi (Lucas, 1846)}

NEW DATA. - Challand-Saint-Victor: Natural Reserve of Lago di Villa, 820 m, grove of reeds, 18.VIII.2011, 1 o $^{7}$, leg. Paschetta \& Giuliano (MRSN-VDA).

Chorotype. - TUM.

Macrohabitat. - Wetlands.

Notes. - New record for Aosta Valley.

\section{*Mendoza canestrinii (Ninni, 1868)}

(Fig. 10)

NEW DATA. - Challand-Saint-Victor: Natural Reserve of Lago di Villa, 820 m, grove of reeds, 16.IX.2011, 1 o", leg. Paschetta \& Giuliano (MRSN-VDA).

Chorotype. - PAL.
Macrohabitat. - Wetlands.

Notes. - New record for Aosta Valley.

\section{- Myrmarachne formicaria (De Geer, 1778)}

Literature. — Pesarini 1997; [Isaia 2005].

CHOROTYPE. - PAL.

Macrohabitat. - Not specified.

Notes. - See notes about Hypsosinga heri.

Neon reticulatus (Blackwall, 1853)

NEW DATA. - Brissogne, Saint-Marcel, Quart, Nus: Natural Reserve of Les Îles, 530 m, riparian forest, 5-29.VII.2011, 2 \% , leg. Paschetta \& Giuliano; Brissogne, Saint-Marcel, Quart, Nus: Natural Reserve of Les Îles, 526 m, riparian forest, 12-26.VII.2013, $20^{7} 1$ \%, leg. Negro; same locality, riparian forest, 26.VII-14.VIII.2013, 2 \%, leg. Negro; same locality, dry meadow, 12-26.VII.2013, 1 \%, leg. Negro.

Literature. - Isaia 2005; [Rossi \& Bosio 2012].

Chorotype. - OLA.

MACROHABITAT. - Riparian habitats, shrublands.

-Pellenes lapponicus (Sundevall, 1833)

Literature. - Calloni 1889.

CHOrotype. - PAL.

Macrohabitat. - Not specified.

NoTes. - Old record in need of validation. The occurrence of the species in Aosta Valley is also considered doubtful by Rossi \& Bosio (2012).

\section{Pellenes tripunctatus (Walckenaer, 1802)}

NEW DATA. - Ayas: Antagnod, 1970-2070 m, alpine pasture, 2007, leg. Franco \& Negro; same locality, ski pistes, 2007, leg. Franco \& Negro.

Literature. — Negro et al. 2009; [Rossi \& Bosio 2012].

CHOROTYPe. - SIE.

MaCROHABITAT. - Alpine pastures, ski pistes.

Philaeus chrysops (Poda, 1761)

(Fig. 11)

NEW DATA. - Sarre-Saint Pierre: Monte Torretta, 900 m, dry meadow, 4-25.VII.2012, 1 \% , leg. Negro (MRSN-VDA); Aymavilles: Pont d'Ael, 800 m, dry meadow, 4-25.VII.2012, 1 o', leg. Negro (MRSN-VDA).

Literature. — Pesarini 1997; [Isaia 2005]; [Rossi \& Bosio 2012]. 
Chorotype. - PAL.

Macrohabitat. - Rocky lands, shrublands.

Notes. - See notes about Agelena labyrinthica.

\section{Phlegra fasciata (Hahn, 1826)}

NEW DATA. - Torgnon: Lo Ditor, 1950 m, peat bog, 11-26.VI.2013, 1 ㅇ, leg. Negro; same locality, 11-26.VI.2013, $10^{\text {" }}$, leg. Negro (MRSN-VDA); Aymavilles: Pont d'Ael, 800 m, dry meadow, 4-25. VII.2012, 1 \%, leg. Negro (MRSN-VDA); Ayas: Antagnod, 19702070 m, 2007, leg. Franco \& Negro.

Literature. — Negro et al. 2009; [Rossi \& Bosio 2012].

Chorotype. - PAL.

MACROHABITAT. - Wetlands, shrublands.

\section{Pseudeuophrys erratica (Walckenaer, 1826)}

Literature. - Fontana et al. 1996; [Isaia 2005]; [Rossi \& Bosio 2012].

Chorotype. - OLA.

MACROHABITAT. - Alpine grasslands, rocky lands.

\section{Pseudeuophrys lanigera (Simon, 1871)}

Literature. - Isaia 2000, [2005]; De Angelis \& Fantoni 2008; [Rossi \& Bosio 2012].

Chorotype. - EUR

MACROHABITAT. — Rocky lands.

Pseudeuophrys vafra (Blackwall, 1867)

LiTERATURE. - Fontana et al. 1996; [Isaia 2005]; [Rossi \& Bosio 2012].

Chorotype. - EUM.

MACrOHABITAT. - Synanthropic habitats.

*Pseudicius encarpatus (Walckenaer, 1802)

NeW Data. - Brissogne, Saint-Marcel, Quart, Nus: Natural Reserve of Les Îles, 530 m, riparian vegetation, 5-29.VII.2011, $10^{\prime \prime}$, leg. Paschetta \& Giuliano; Morgex-La Salle: Natural Reserve of Marais, $900 \mathrm{~m}$, meadow-grove of reeds, 9.VII.2012, $10^{7}$, leg. Paschetta.

CHOROtype. - EUR.

MACROHABITAT. - Riparian habitats, wetlands.

Notes. - New record for Aosta Valley.

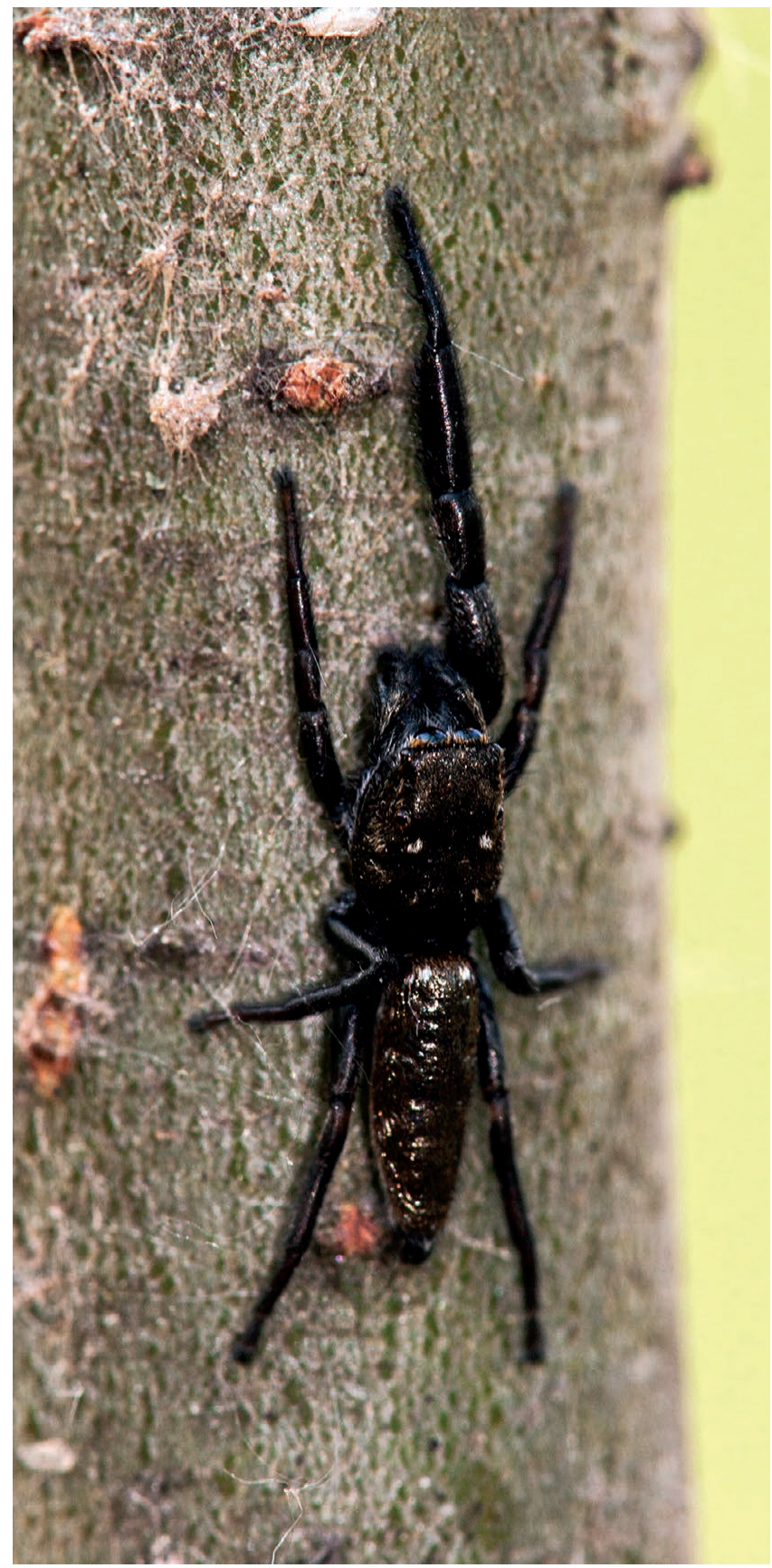

FIG. 10. - Mendoza canestrinii (Ninni, 1868) (Saltcidae), male. Average body length: 8.3-10.3 mm. Challand-Saint-Victor: Natural Reserve of Lago di Villa. Photograph: Mauro Paschetta 2009.

\section{Saitis barbipes (Simon, 1868)}

NeW DATA. - Pont-Saint-Martin: Natural Reserve of Stagno di Holay, $679 \mathrm{~m}$, broadleaved forest (Castanea sativa), 11-26.VI.2013, 1 o", leg. Negro; same locality, 26.VI-12.VII.2013, $10^{7}$, leg. Negro; same locality, alpine grassland, 26.VII-14.VIII.2013, 1 \% , leg. Negro; Challand-Saint-Victor: Natural Reserve of Lago di Villa, $820 \mathrm{~m}$, broadleaved forest, 11-26.VI.2013, 1 o $^{7}$, leg. Negro, (MRSN-VDA).

Literature. — Pesarini 1997; [Isaia 2005].

Chorotype. - MED 
MACROHABITAT. - Broadleaved forests, alpine grasslands.

Notes. - See notes about Agelena labyrinthica.

- Salticus cingulatus (Panzer, 1797)

Literature. — Rossi \& Bosio 2012.

CHOROTYPe. - SIE.

MacrohabitAT. - Broadleaved forests.

Notes. - See Tegenaria domestica.

\section{Salticus scenicus (Clerck, 1757)}

NEW DATA. - La Thuile: Comba Thuilette, 2000 m, rocky land, 13.IX.2012, 1 \%, leg. Paschetta, Mammola \& Marguerettaz; Ayas: Antagnod, 1970-2070 m, alpine pasture, leg. Franco \& Negro.

Literature. - Calloni 1889; Fontana et al. 1996; [Isaia 2005]; [Rossi \& Bosio 2012].

Chorotype. - OLA.

MACROHABITAT. - Rocky lands, alpine pastures, synanthropic habitats.

Salticus zebraneus (C. L. Koch, 1837)

Literature. — Isaia 2005; [Rossi \& Bosio 2012].

Chorotype. - PAL.

MacrohabitAT. - Not specified.

Sitticus longipes (Canestrini, 1873)

NEW DATA. — Courmayeur: Val Ferret, 1700 m, peat bog, 11.VII.2012, 1 ơ, leg. Paschetta \& Giuliano \& Mammola.

LiTerATURE. - Calloni 1889; Isaia 2000; [Isaia 2005]; Negro et al. 2010, 2013 [Rossi \& Bosio 2012].

Chorotype. - EUR.

MACROHABITAT. — Rocky lands, ski pistes, wetlands.

\section{Sitticus rupicola (C. L. Koch, 1837)}

NEW DATA. - Fontainemore, Monte Mars, 1900 m, rocky land, 17.IX.2012, 2 o", leg. Paschetta \& Marguerettaz.

LITERATURE. - Calloni 1889; [Isaia 2005].

Chorotype. - EUR.

MACROHABITAT. - Rocky lands.

Notes. - The occurrence of this species in Aosta Valley, here confirmed by original data, was considered doubtful by Rossi \& Bosio (2012).
Sitticus saxicola (C. L. Koch, 1846)

NeW DATA. - La Thuile: Comba Thuilette, 2000 m, rocky land, 13.IX.2012, 1 \%, leg. Paschetta, Mammola \& Marguerettaz; same locality, 10.VII.2012, 1 o $^{7} 5$ \%, leg. Paschetta, Giuliano, Mammola \& Marguerettaz.

LiTERATURE. - Isaia 2000; [Isaia 2005]; Negro et al. 2009; [Rossi \& Bosio 2012].

Chorotype. - WPA.

MACROHABITAT. - Rocky lands.

Talavera aequipes (O. P.-Cambridge, 1871)

LiterATURE. - Isaia 2005; [Rossi \& Bosio 2012].

Chorotype. - PAL.

Macrohabitat. - Not specified.

Family SCYTODIDAE Blackwall, 1864

Scytodes thoracica (Latreille, 1802)

NEW DATA. - Saint Denis: Brison-Cly, $1000 \mathrm{~m}$, broadleaved forest,

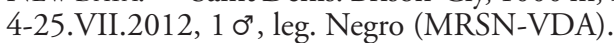

Literature. - Isaia 2005; [Rossi \& Bosio 2012].

Chorotype. - OLA.

MACROHABITAT. - Synanthropic habitats, broadleaved forests.

Family SEGESTRIIDAE Simon, 1893

Segestria bavarica C. L. Koch, 1843

LiTERATURE. - Isaia 2005; [Rossi \& Bosio 2012].

Chorotype. - PAL.

MACROHABITAT. - Synanthropic habitats.

Segestria senoculata (Linnaeus, 1758)

Literature. - Pavesi 1904; Isaia 2000; De Angelis \& Fantoni 2008; Negro et al. 2009; [Rossi \& Bosio 2012].

Chorotype. - PAL.

MACROHABITAT. - Broadleaved forests.

Family SPARASSIDAE Bertkau, 1872

Micrommata virescens (Clerck, 1757)

(Fig. 12)

NEW DATA. - La Thuile: Comba Thuilette, $2000 \mathrm{~m}$, peat bog, 10.VII.2012, 1 ơ, leg. Paschetta, Giuliano, Mammola \& Margueret- 


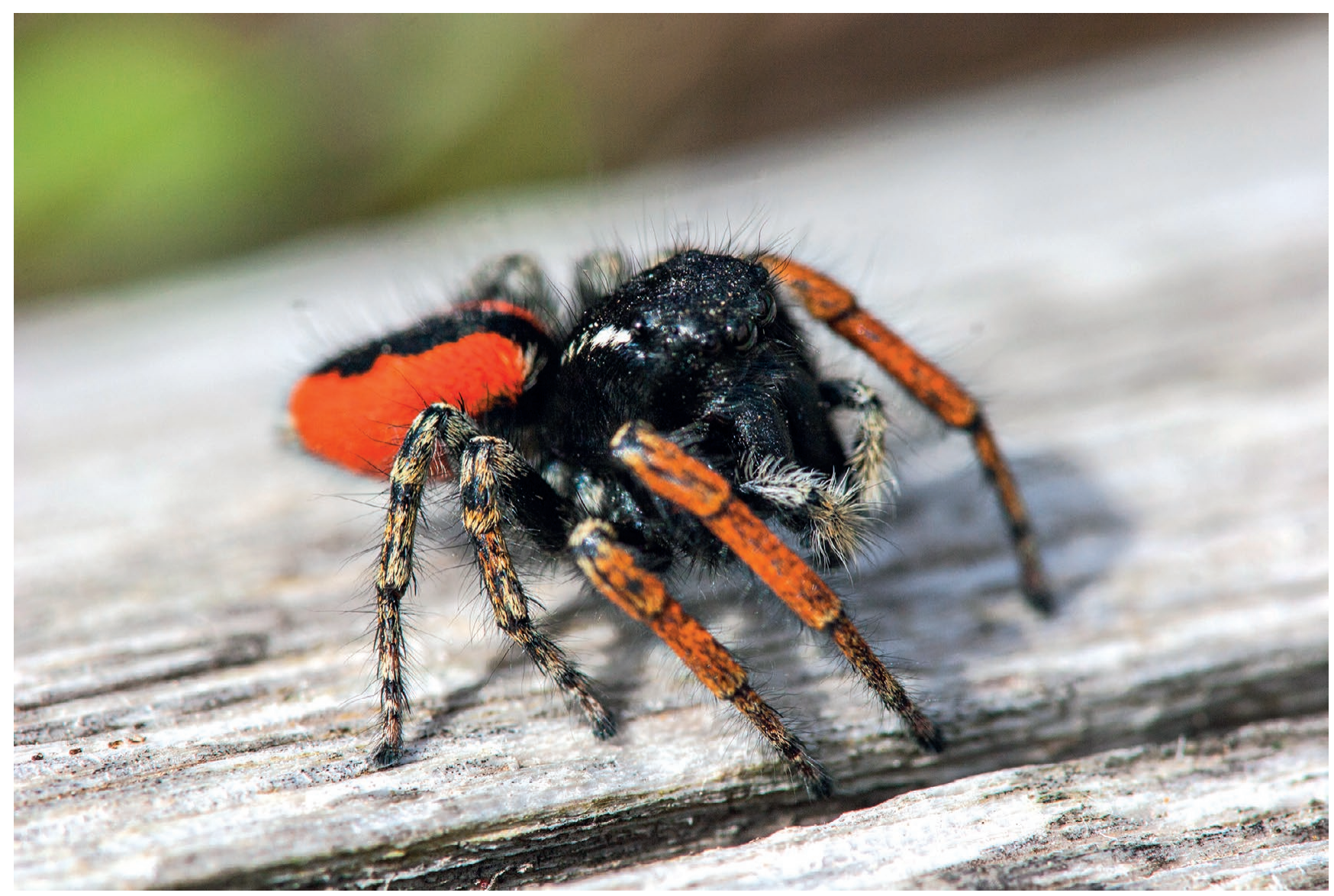

FIG. 11. - Philaeus chrysops (Poda, 1761) (Salticidae), male. Average body length of the male: 5.4-9.5 mm. Sarre-Saint Pierre: Monte Torretta. Photograph: Mauro Paschetta 2011.

taz; same locality, wet meadow, 16.VIII.2011, 1 ㅇ, leg. Paschetta \& Giuliano; Challand-Saint-Victor: Natural Reserve of Lago di Villa, 820 m, grove of reeds, 18.VIII.2011, 1 \%, leg. Paschetta, Giuliano.

Literature. - Isaia 2005; [Rossi \& Bosio 2012].

CHOROTYPe. - PAL.

MACROHABITAT. - Wetlands.

\section{Family Tetragnathidae Menge, 1866}

Meta menardi (Latreille, 1804)

LiterATURE. — Arnò \& Lana 2005; [Isaia 2005]; [Rossi \& Bosio 2012].

CHOROTYPE. - PAL.

Macrohabitat. - Caves.

\section{Metellina mengei (Blackwall, 1870)}

LiteratURE. — Isaia 2000, [2005]; [Rossi \& Bosio 2012].

ChOrotype. - EUR.

MACROHABITAT. - Synanthropic habitats, broadleaved forests.
Metellina merianae (Scopoli, 1763)

(Fig. 13)

NEW DATA. - Arvier: Lago di Lolair, 1175 m, wet meadow, 18.VIII16.IX.2011, 1 \%, leg. Paschetta \& Giuliano.

LitERATURE. — Isaia 2000; Arnò \& Lana 2005; [Rossi \& Bosio 2012].

Chorotype. - EUR.

MACROHABITAT. - Wetlands, caves, coniferous forests, synanthropic habitats.

\section{Metellina segmentata (Clerck, 1757)}

NEW DATA. - Pont-Saint-Martin: Natural Reserve of Stagno di Holay, 775 m, riparian vegetation, 16.IX.2011, 1 o $^{7} 1$ ㅇ, leg. Paschetta \& Giuliano; Challand-Saint-Victor: Natural Reserve of Lago di Villa, 820 m, grove of reeds, 14.IX.2012, 4 ㅇ, leg. Paschetta \& Mammola; same locality, riparian vegetation, 16.IX.2011, $10^{\prime \prime}$ 1 \%, leg. Paschetta \& Giuliano; Courmayeur: Val Ferret, 1630 m, shrubs, 26.VI-12.VII.2013, 1 ○, leg. Negro; Brissogne, Saint-Marcel, Quart, Nus: Natural Reserve of Les Îles, $530 \mathrm{~m}$, riparian vegetation, 17.VIII.2011, 1 \% , leg. Paschetta \& Giuliano.

Literature. - Rossi \& Arnò 1995; [Isaia 2005]; [Rossi \& Bosio 2012]. 
Chorotype. - OLA.

MACROHABITAT. - Synanthropic habitats, riparian habitats, wetlands, shrublands.

\section{Pachygnatha clercki Sundevall, 1823}

NEW DATA. - Arvier: Lago di Lolair, 1175 m, wet meadow, 29.VII18.VIII.2011, 2 o" 1 \%, leg. Paschetta \& Giuliano; same locality: 18.VIII-16.IX.2011, 1 \%, leg. Paschetta \& Giuliano; Morgex-La Salle: Natural Reserve of Marais, 896 m, grove of reeds, 12-26. VII.2013, 1 \% , leg. Negro; Verrayes: stagno di Loson, $1520 \mathrm{~m}$, grove of reeds/peat bog, 13.VI-4.VII.2011, 1 Ơ $^{7}$, leg. Paschetta \& Giuliano (MRSN-VDA).

LiterATURE. — Rossi \& Arnò 1995; [Isaia 2005]; [Rossi \& Bosio 2012].

Chorotype. - OLA.

MACROHABITAT. - Wetlands, shrublands.

\section{Pachygnatha degeeri Sundevall, 1830}

NEW DATA. - Pont-Saint-Martin: Natural Reserve of Stagno di Holay, 616 m, alpine grassland, 11-26.VI.2013, 1 o", leg. Negro (MRSN-VDA); Arvier: Lago di Lolair, 1175 m, wet meadow, 5-29. VII.2011, 1 o", leg. Paschetta \& Giuliano; same locality, 18.VIII16.IX.2011, 1 ơ, leg. Paschetta \& Giuliano; Torgnon: Lo Ditor, $1950 \mathrm{~m}$, peat bog, 26.VII-14.VIII.2013, 1 ơ, leg. Negro; Morgex-La Salle: Natural Reserve of Marais, 896 m, grove of reeds, 26.VI-12. VII.2013, 1 0’, leg. Negro.

Literature. — Negro et al. 2009; [Rossi \& Bosio 2012].

Chorotype. - PAL.

MACROHABITAT. - Alpine grasslands, wetlands.

\section{Pachygnatha listeri Sundevall, 1830}

NEW DATA. - Morgex-La Salle: Natural Reserve of Marais, 896 m, riparian forest, 11-26.VI.2013, $10^{\prime \prime}$, leg. Negro (MRSN-VDA).

LiterATURE. — Rossi \& Arnò 1995; [Isaia 2005]; Negro et al. 2009; [Rossi \& Bosio 2012].

Chorotype. - PAL.

MACROHABITAT. - Shrublands, riparian habitats, broadleaved forests, wetlands.

\section{Tetragnatha extensa (Linnaeus, 1758)}

NEW DATA. - Challand-Saint-Victor: Natural Reserve of Lago di Villa, 820 m, grove of reeds, 18.VIII.2011, 1 ơ $^{\text {1 }}$ ㅇ, leg. Paschetta \& Giuliano (MRSN-VDA); La Thuile: Comba Thuilette, $2000 \mathrm{~m}$, riparian vegetation, 16.VIII.2011, 1 \%, leg. Paschetta \& Giuliano.

Literature. — Rossi \& Arnò 1995; [Isaia 2005]; [Rossi \& Bosio 2012].

Chorotype. - OLA.

MACROHABITAT. - Shrublands, synanthropic habitats, riparian habitats, wetlands.

\section{Tetragnatha montana Simon, 1874}

NEW DATA. - Morgex-La Salle: Natural Reserve of Marais, 900 m, riparian vegetation, 16.VIII.2011, 2 \% leg. Paschetta, Giuliano; same locality, meadow-grove of reeds, 9.VII.2012, $20^{\circ} 2$ \% , leg. Paschetta; same locality, broadleaved forest, 9.VII.2012, 1 , leg. Paschetta; Challand-Saint-Victor: Natural Reserve of Lago di Villa, $820 \mathrm{~m}$, riparian vegetation, 18.VIII.2011, 2 \%, leg. Paschetta \& Giuliano (MRSN-VDA); Pont-Saint-Martin: Natural Reserve of Stagno di Holay, 775 m, wet meadow, 13.VI-4.VII.2011, 1 o, leg. Paschetta \& Giuliano; same locality, riparian vegetation, 13.VIII.2012, 1 \% , leg. Paschetta.

Literature. - Rossi \& Arnò 1995; [Isaia 2005]; [Rossi \& Bosio 2012].

Chorotype. - PAL.

MACROHABITAT. - Riparian habitats, wetlands, broadleaved forests.

•Tetragnatha obtusa C. L. Koch, 1837

Literature. - Rossi \& Bosio 2012.

Chorotype. - PAL.

MacrohabitaT. - Riparian habitats.

Notes. - See Tegenaria domestica.

-Tetragnatha pinicola L. Koch, 1870

LiterATURE. - Rossi \& Bosio 2012.

Chorotype. - PAL.

Macrohabitat. - Coniferous forests.

Notes. - See Tegenaria domestica.

Family THERIDIIDAE Sundevall, 1833

$$
\text { Asagena phalerata (Panzer, 1801) }
$$

NEW DATA. - Torgnon: Lo Ditor, 1950 m, peat bog, 26.VI-12. VII.2013, 4 o", leg. Negro; same locality, 26.VII-14.VIII.2013, $10^{7}$ 1 , leg. Negro; Challand-Saint-Victor: Natural Reserve of Lago di Villa, 820 m, broadleaved forest, 11-26.VI.2013, 1 o", leg. Negro; Ayas: Antagnod, 1970-2070 m, alpine pasture, 2007, leg. Franco \& Negro; same locality, ski pistes, 2007, leg. Franco \& Negro.

LiTERATURE. - Calloni 1889; di Caporiacco 1928; [Isaia 2005]; De Angelis \& Fantoni 2008; Negro et al. 2009, 2010, 2013 [Rossi \& Bosio 2012].

Chorotype. - PAL.

MACROHABITAT. - Wetlands, alpine prairies, alpine pastures, ski pistes, broadleaved forests.

\section{Crustulina guttata (Wider, 1834)}

NEW DATA. - Pont-Saint-Martin: Natural Reserve of Stagno di Holay, 679 m, broadleaved forest (Castanea sativa), 12-26.VII.2013, 


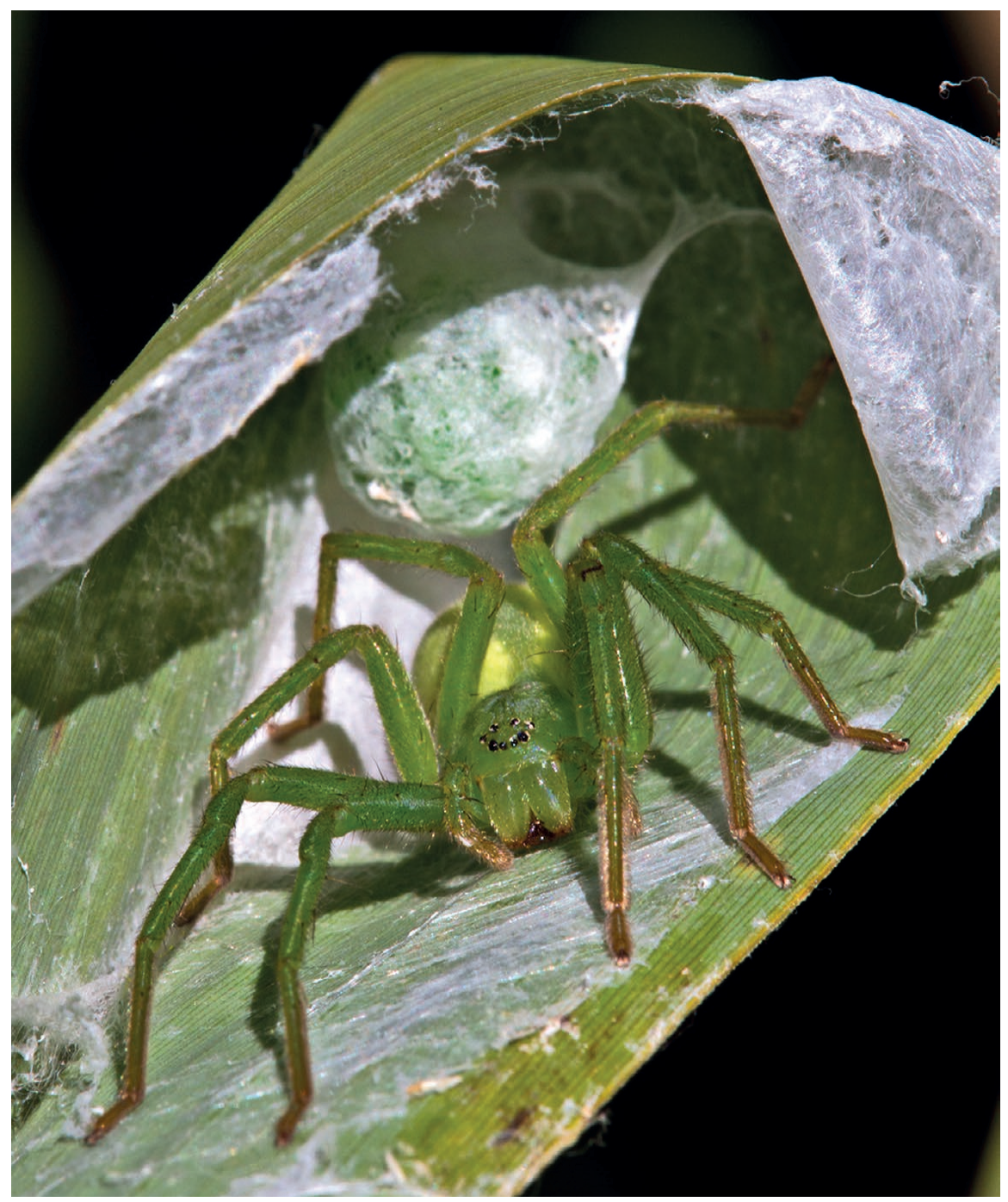

FIG. 12. - Micrommata virescens (Clerck, 1757) (Sparassidae), female. Average body length of the female: 12-16.6 mm. Challand-Saint-Victor: Natural Reserve of Lago di Villa. Photograph: Mauro Paschetta 2011.

1 ㅇ, leg. Negro; same locality, 11-26.VI.2013, 1 ơ, leg. Negro (MRSN-VDA); Ayas: Antagnod, 1970-2070 m, alpine pasture, 2007, leg. Franco \& Negro.

LITERATURE. — Calloni 1889; [Isaia 2005].

CHorotype. - PAL.

MACROHABITAT. - Broadleaved forests, alpine pasture.

Notes. - The occurrence of this species in Aosta Valley, here confirmed by original data, was considered doubtful by Rossi $\&$ Bosio (2012).

*Dipoena melanogaster (C. L. Koch, 1837)

NEW DATA. - Brissogne, Saint-Marcel, Quart, Nus: Natural Reserve of Les Îles, $530 \mathrm{~m}$, dry meadow, 18.VIII-16.IX.2011, 1 o', $^{\text {, }}$ leg. Paschetta \& Giuliano.

CHOROTYPe. - EUR.
MACROHABITAT. — Shrublands.

Notes. - New record for Aosta Valley.

\section{Dipoena torva (Thorell, 1875)}

Literature. - Isaia 2000, [2005]; [Rossi \& Bosio 2012].

Chorotype. - OLA.

Macrohabitat. - Broadleaved forests.

\section{Enoplognatha ovata (Clerck, 1757)} vel Enopognatha latimana Hippa \& Oksala, 1982

NEW DATA. - Morgex-La Salle: Natural Reserve of Marais, 900 m, broadleaved forest, 9.VII.2012, 1 \%, leg. Paschetta; same locality, meadow-grove of reeds, 9.VII.2012, $10^{\circ}$, leg. Paschetta; same locality, riparian vegetation, 16.VIII.2011, 2 \% , leg. Paschetta, Giuliano; 
same locality, grove of reeds, 26.VII-14.VIII.2013, 1 o", leg. Negro; Brissogne, Saint-Marcel, Quart, Nus: Natural Reserve of Les Illes, 526 m, riparian forest, 11-26.VI.2013, 1 ơ $^{\prime \prime}$ leg. Negro; PontSaint-Martin: Natural Reserve of Stagno di Holay, 775 m, riparian vegetation, 13.VIII.2012, 1 ㅇ, leg. Paschetta.

Literature. - Rossi \& Arnò 1995; [Isaia 2005]; [Rossi \& Bosio 2012].

Chorotype. - OLA.

MACROHABITAT. - Broadleaved forests, wetlands, riparian habitats.

\section{Enoplognatha thoracica (Hahn, 1833)}

NEW DATA. - Brissogne, Saint-Marcel, Quart, Nus: Natural Reserve of Les Îles, 530 m, dry meadow, 29.VII-18.VIII.2011, 1 \%, leg. Paschetta \& Giuliano.

Literature. - Negro et al. 2009; [Rossi \& Bosio 2012].

Chorotype. - OLA.

MACROHABITAT. - Shrublands, alpine prairies.

\section{Episinus maculipes Cavanna, 1876}

NEW DATA. — Ayas: Antagnod, 1970-2070 m, alpine prairies, 2007 leg. Franco \& Negro.

Literature. - De Angelis \& Fantoni 2008; [Rossi \& Bosio 2012]. Chorotype. - SEU.

MACROHABITAT. - Alpine prairies.

\section{Episinus truncatus Latreille, 1809}

(Fig. 14)

NeW DATA. - Brissogne, Saint-Marcel, Quart, Nus: Natural Reserve of Les Îles, 526 m, riparian forest, 26.VI-12.VII.2013, 1 o, leg. Negro; Sarre-Saint Pierre: Monte Torretta, $900 \mathrm{~m}$, broadleaved forest, 4-25.VII.2012, 1 o", leg. Negro; ChallandSaint-Victor: Natural Reserve of Lago di Villa, $820 \mathrm{~m}$, broadleaved forest, 11-26.VI.2013, $10^{7}$, leg. Negro; same locality, 26.VI-12.VII.2013, 9 o' 2 \%, leg. Negro; same locality, 12-26. VII.2013, 1 ơ, leg. Negro; same locality, 26.VII-14.VIII.2013, 2 ơ, leg. Negro; same locality, 14-28.VIII.2013, 1 \&, leg. Negro; Pont-Saint-Martin: Natural Reserve of Stagno di Holay, 616 m, alpine grassland, 26.VI-12.VII.2013, $10^{7}$, leg. Negro; same locality, 26.VII-14.VIII.2013, 1 ơ 2 우, leg. Negro; same locality, 14-28.VIII.2013, 3 o' 1 o, leg. Negro; Aymavilles: Pont d'Ael, $800 \mathrm{~m}$, dry meadow, 13.VI-4.VII.2012, 1 ㅇ, leg. Negro; same locality, 25.VII-20.VIII.2012, 1 \% leg. Negro (MRSN-VDA); same locality, 25.VII-20.VIII.2012, 4 \% , leg. Negro; Aymavilles: Pont d'Ael, $800 \mathrm{~m}$, broadleaved forest, 13.VI-4.VII.2012, $1 \mathrm{O}^{\circ}$, leg. Negro; same locality, 25.VII-20.VIII.2012, 1 o $^{7} 2$ ㅇ, leg. Negro; Gressan: Natural Reserve of Côte de Gargantua, 700 m, broadleaved forest, 4-25.VII.2012, 2 \%, leg. Negro; same locality, dry meadow, 4-25.VII.2012, 1 \%, leg. Negro; Morgex-La Salle: Natural Reserve of Marais, 896 m, grove of reeds, 26.VII-14. VIII.2013, 1 ㅇ, leg. Negro.

Literature. — De Angelis \& Fantoni 2008; [Rossi \& Bosio 2012].
Chorotype. - EUR.

MacrohabitaT. - Riparian habitats, broadleaved forests, shrublands, wetlands, alpine grasslands.

Euryopis flavomaculata (C. L. Koch, 1836)

NEW DATA. - Brissogne, Saint-Marcel, Quart, Nus: Natural Reserve of Les Îles, $530 \mathrm{~m}$, riparian vegetation, 14.VI-5.VII.2011, 3 o $^{7} 8$ 우, leg. Paschetta \& Giuliano; same locality, 14.VI-5.VII.2011, 2 운,

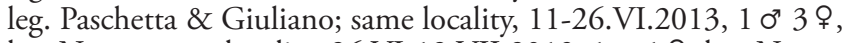
leg. Negro; same locality, 26.VI-12.VII.2013, 1 ơ 1 \&, leg. Negro; Morgex-La Salle: Natural Reserve of Marais, 896 m, riparian forest, 11-26.VI.2013, 2 o", leg. Negro (MRSN-VDA); same locality, 26.VI-12.VII.2013, 1 o", leg. Negro.

Literature. — Negro et al. 2009; [Rossi \& Bosio 2012].

Chorotype. - PAL.

MACROHABITAT. - Riparian habitats, alpine prairies.

\section{Parasteatoda lunata (Clerck, 1757)}

NeW DATA. - La Thuile: Comba Thuilette, 2000 m, wet meadow, 16.VIII.2011, 1 ơ, leg. Paschetta \& Giuliano (MRSN-VDA).

Literature. - Isaia 2005; [Rossi \& Bosio 2012].

Chorotype. - PAL.

MaCrohabitAT. - Wetlands.

Parasteatoda tepidariorum (C. L. Koch, 1841)

Literature. - Isaia 2005; [Rossi \& Bosio 2012].

Chorotype. - COS.

Macrohabitat. - Not specified.

\section{Pholcomma gibbum (Westring, 1851)}

Literature. - Pesarini 2000; [Isaia 2005]; [Rossi \& Bosio 2012].

CHOrotype. - EUR.

Macrohabitat. - Not specified.

\section{Phylloneta impressa (L. Koch, 1881)}

NEW DATA. - Fontainemore, Monte Mars, 1900 m, peat bog, 14.IX.2012, 1 o, leg. Paschetta \& Mammola; Pont-Saint-Martin: Natural Reserve of Stagno di Holay, 775 m, riparian vegetation, 13.VIII.2012, 1 \%, leg. Paschetta; La Thuile: Comba Thuilette, 2000 m, wet meadow, 9.VII.2012, 1 o 1 ㅇ, leg. Paschetta; same locality, 16.VIII.2011, 1 \%, leg. Paschetta \& Giuliano; same locality, 17.VIII.2011, 2 o, leg. Paschetta \& Giuliano; same locality, peat bog, 10.VII.2012, 2 \%, leg. Paschetta \& Giuliano, Mammola \& Marguerettaz; same locality, 13.IX.2012, 1 \&, leg. Paschetta, Mammola \& Marguerettaz. 


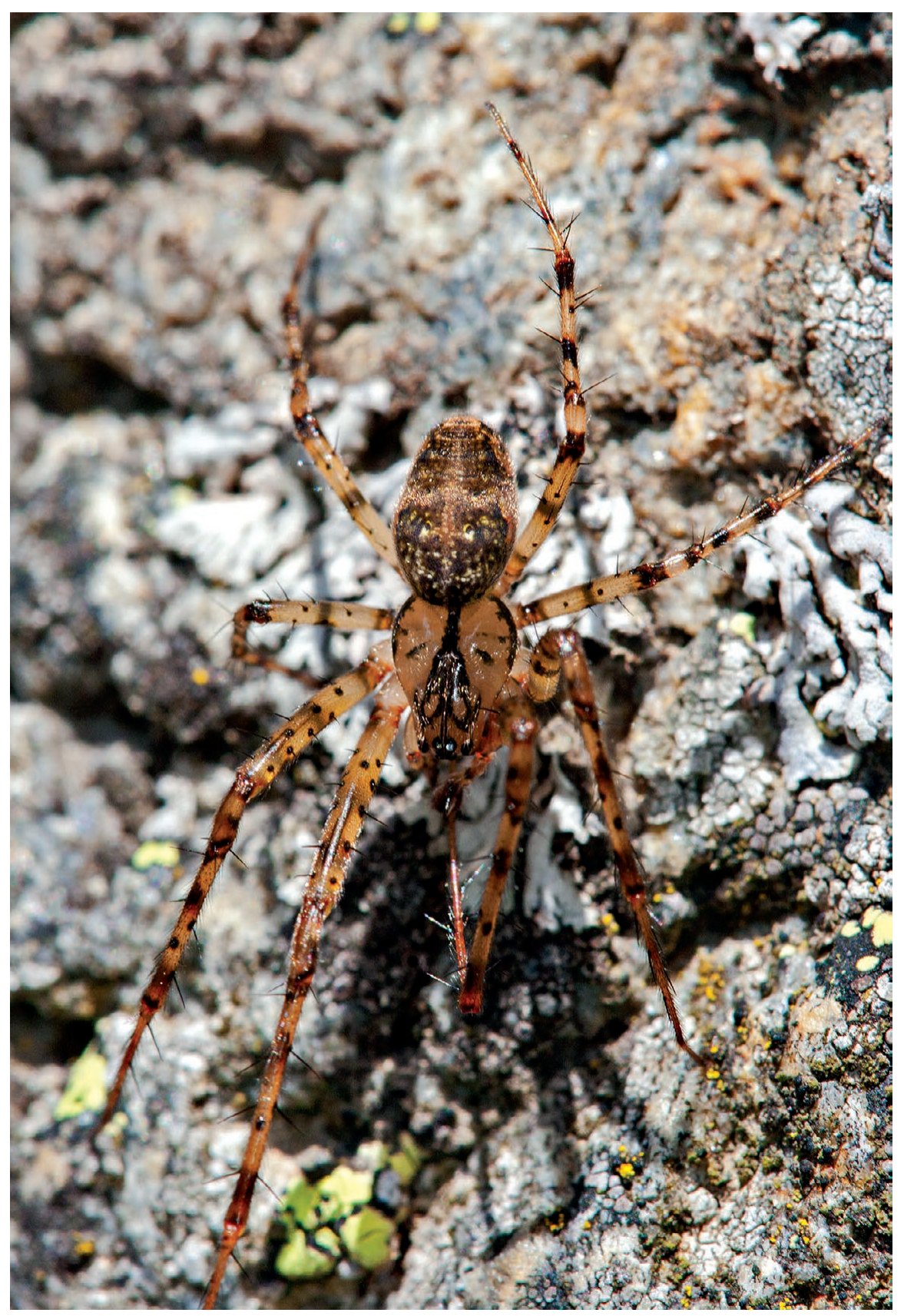

FIG. 13. - Metellina merianae (Scopoli, 1763) (Tetragnathidae), female. Average body length of the female: 7.3-12 mm. Arvier: Lago di Lolair. Photograph: Mauro Paschetta 2011.

LiterATURE. — Rossi \& Arnò 1995; Isaia 2000, [2005]; [Rossi \& Bosio 2012].

CHorotype. - OLA.

MACROHABITAT. - Riparian habitats, wetlands, coniferous forests, broadleaved forests, shrublands.

\section{Phylloneta sisyphia (Clerck, 1757)}

NEW DATA. - Courmayeur: Val Ferret, $1700 \mathrm{~m}$, grove of reeds, 11.VII.2012, 2 ㅇ, leg. Paschetta, Giuliano \& Mammola; same locality, peat bog, 11.VII.2012, 1 \%, leg. Paschetta, Giuliano \& Mammola; Morgex-La Salle: Natural Reserve of Marais, $900 \mathrm{~m}$, grove of reeds, 9.VII.2012, 1 \&, leg. Paschetta.

LiTERATURE. — Calloni 1889; Isaia 2000, [2005]; [Rossi \& Bosio 2012].

Chorotype. - PAL.

Macrohabitat. - Wetlands, coniferous forests. 
Robertus arundineti (O. P.-Cambridge, 1871)

LITERATURe. - Isaia 2000, [2005]; [Rossi \& Bosio 2012].

CHOROTYPe. - PAL.

MACROHABITAT. - Broadleaved forests.

Robertus lividus (Blackwall, 1836)

NEW DATA. - Challand-Saint-Victor: Natural Reserve of Lago di Villa, 820 m, broadleaved forest, 11-26.VI.2013, 1 o', leg. Negro; same locality, 26.VI-12.VII.2013, 2 o", leg.Negro (MRSN-VDA); same locality, wet meadow, 13.VI-4.VII.2011, $10^{7}$, leg. Paschetta \& Giuliano; same locality, riparian forest, 13.VI-4.VII.2011, $10^{7}$, leg. Paschetta \& Giuliano; Morgex-La Salle: Natural Reserve of

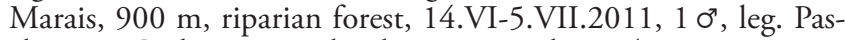
chetta \& Giuliano; same locality, wet meadow, 14.VI-5.VII.2011, $10^{7}$, leg. Paschetta \& Giuliano; same locality, 18/X/2011-16.IX.2011, $10^{7} 1 \%$, leg. Paschetta \& Giuliano; Brissogne, Saint-Marcel, Quart, Nus: Natural Reserve of Les Îles, 530 m, riparian forest, 14.VI-5. VII.2011, 1 ơ $^{\text {1 }} 1$ \% , leg. Paschetta \& Giuliano; same locality, 5-29. VII.2011, $10^{7}$, leg. Paschetta \& Giuliano; same locality, 26.VI-12. VII.2013, 1 o", leg. Negro; Torgnon: Lo Ditor, $1950 \mathrm{~m}$, peat bog, 26.VII-14.VIII.2013, 1 ơ, leg. Negro; Arvier: Lago di Lolair, 1175 m, wet meadow, 14.VI-5.VII.2011, 1 \%, leg. Paschetta \& Giuliano.

Literature. - Calloni 1889; Pavesi 1904; De Angelis \& Fantoni 2008; Negro et al. 2009; [Rossi \& Bosio 2012].

Chorotype. - OLA.

MACROHABITAT. - Broadleaved forests, wetlands, riparian habitats.

Robertus neglectus (O. P.-Cambridge, 1871)

NeW Data. - Brissogne, Saint-Marcel, Quart, Nus: Natural Reserve of Les Îles, 530 m, riparian forest, 5-29.VII.2011, 1 , leg. Paschetta \& Giuliano.

Literature. — Negro et al. 2009; [Rossi \& Bosio 2012].

Chorotype. - SIE.

MACROHABITAT. - Riparian habitats, alpine prairies.

${ }^{*}$ Robertus scoticus Jackson, 1914

NEW DATA. - Courmayeur: Val Ferret, 1630 m, shrubs, 14-28. VIII.2013, 1 ơ $^{\pi}$ leg. Negro.

Chorotype. - EUR.

MACROHABITAT. - Shrublands.

Notes. - New record for Aosta Valley.

Robertus truncorum (L. Koch, 1872)

Literature. — Negro et al. 2009; [Rossi \& Bosio 2012].

Chorotype. - CEU.

Macrohabitat. - Coniferous forests, alpine prairies.
Rugathodes bellicosus (Simon, 1873)

Literature. — Gobbi et al. 2010; [Rossi \& Bosio 2012].

CHOROTYPe. - SIE.

MacrohabitaT. — Rocky lands.

\section{Steatoda albomaculata (De Geer, 1778)}

NEW DATA. - Brissogne, Saint-Marcel, Quart, Nus: Natural Reserve of Les Îles, 526 m, dry meadow, 11-26.VI.2013, 1 \%, leg. Negro (MRSN-VDA).

LITERATURE. — Calloni 1889; [Isaia 2005].

Chorotype. - COS.

MACROHABITAT. - Shrublands.

Notes. - The occurrence of this species in Aosta Valley, here confirmed by original data, was considered doubtful by Rossi \& Bosio (2012).

\section{Steatoda bipunctata (Linnaeus, 1758)}

LiTERATURE. - Isaia 2005; [Rossi \& Bosio 2012].

Chorotype. - OLA.

MACROHABITAT. - Synanthropic habitats.

- Steatoda grossa (C. L. Koch, 1838)

Literature. — Rossi \& Bosio 2012.

Chorotype. - COS.

MACROHABITAT. - Synanthropic habitats.

Notes. - See Tegenaria domestica.

Steatoda triangulosa (Walckenaer, 1802)

LiterATURE. - Isaia 2005; [Rossi \& Bosio 2012].

Chorotype. - COS.

MacrohabitaT. - Synanthropic habitats.

Theridion melanurum Hahn, 1831

Literature. — Pavesi 1904; [Isaia 2005]; [Rossi \& Bosio 2012].

Chorotype. - OLA.

MaCrohabitaT. - Synanthropic habitats. 


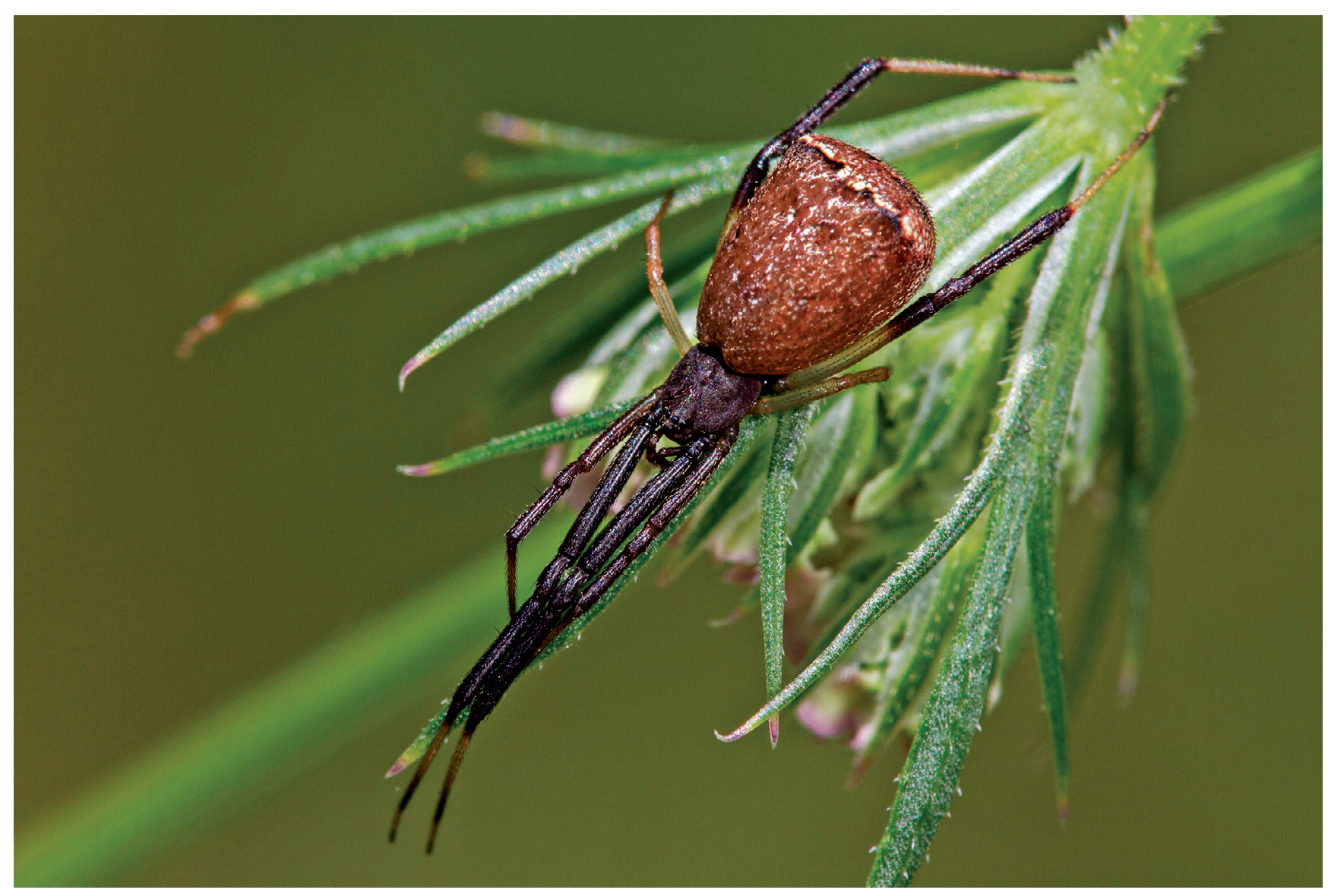

FIG. 14. - Episinus truncatus Latreille, 1809 (Theridiidae), female. Average body length of the female: 5-6.2 mm. Challand-Saint-Victor: Natural Reserve of Lago di Villa. Photograph: Mauro Paschetta 2011.

\section{Theridion petraeum L. Koch, 1872}

NEW DATA. - Fontainemore, Monte Mars, 1900 m, Pietraia, 14.IX.2012, 1 \%, leg. Paschetta \& Mammola; Fontainemore, Monte Mars, 1900 m, rocky land, 17.IX.2012, 1 ơ 2 \%, leg. Paschetta \& Marguerettaz.

Literature. - Di Caporiacco 1928; [Isaia 2005]; [Rossi \& Bosio 2012].

Chorotype. - OLA.

MACROHABITAT. - Rocky lands.

\section{Theridion pictum (Walckenaer, 1802)}

LiterATURE. - Rossi \& Bosio 2012.

Chorotype. - OLA.

MACrOHABitaT. - Synanthropic habitats.

Theridion varians Hahn, 1833

NEW DATA. - Morgex-La Salle: Natural Reserve of Marais, 900 m, meadow-grove of reeds, 9.VII.2012, 1 \%, leg. Paschetta.
Literature. - Rossi \& Arnò 1995; [Isaia 2005]; [Rossi \& Bosio 2012].

Chorotype. - OLA.

MACROHABITAT. - Broadleaved forests, wetlands.

Family THOMISIDAE Sundevall, 1833

Cozyptila blackwalli (Simon, 1875)

NEW DATA. - Saint Christophe: Tsatelet, $750 \mathrm{~m}$, broadleaved

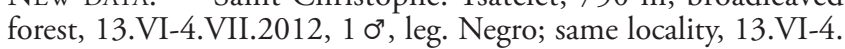
VII.2012, $10^{\pi}$, leg. Negro (MRSN-VDA).

LiterATURE. - Isaia 2005; [Rossi \& Bosio 2012].

CHOROTYPe. - SIE.

MACROHABITAT. - Broadleaved forests.

\section{Diaea dorsata (Fabricius, 1777)}

Literature. — Isaia 2005; [Rossi \& Bosio 2012]. 
Chorotype. - SIE.

MacrohabitaT. — Not specified.

Ebrechtella tricuspidata (Fabricius, 1775)

(Fig. 15)

Literature. - Rossi \& Arnò 1995; [Isaia 2005]; [Rossi \& Bosio 2012].

Chorotype. - PAL.

MACROHABITAT. - Shrublands, broadleaved forests.

Heriaeus hirtus (Latreille, 1819)

Literature. — Isaia 2005; [Rossi \& Bosio 2012].

CHOrotype. - EUR.

MACrOHABiTAT. - Synanthropic habitats.

Misumena vatia (Clerck, 1757)

Literature. - Isaia 2005; [Rossi \& Bosio 2012].

Chorotype. - OLA.

MacrohabitaT. - Not specified.

\section{Ozyptila atomaria (Panzer, 1801)}

NEW DATA. - Gressan: Natural Reserve of Côte de Gargantua, 700 m, dry meadow, 3-24.V.2012, 1 o", leg. Negro (MRSN-VDA); Torgnon: Lo Ditor, 1950 m, peat bog, 11-26.VI.2013, 1 ㅇ, leg. Negro (MRSN-VDA).

LiTERATURE. - Di Caporiacco 1928; [Isaia 2005]; [Rossi \& Bosio 2012].

Chorotype. - PAL.

MACROHABITAT. - Shrublands, wetlands.

\section{Ozyptila praticola (C. L. Koch, 1837)}

NEW DATA. - Brissogne, Saint-Marcel, Quart, Nus: Natural Reserve of Les Îles, $530 \mathrm{~m}$, riparian vegetation, 14.VI-5.VII.2011, 66 o", leg. Paschetta \& Giuliano; same locality, 5-29.VII.2011, $50^{77} 4$ 우, leg. Paschetta \& Giuliano; same locality, 29.VII-18.VIII.2011, 3 o" 2 , leg. Paschetta \& Giuliano; same locality, 18.VIII-16.IX.2011, 7 o' 4 \% , leg. Paschetta \& Giuliano; same locality, 11-26.VI.2013, 24 o", leg. Negro; same locality, 26.VI-12.VII.2013, 12 o", leg. Negro; same locality, 12-26.VII.2013, $20^{\text {" }}$, leg. Negro; same locality, 26.VII-14.VIII.2013, 2 o 2 9, leg. Negro; same locality, 14-28. VIII.2013, 2 o", leg. Negro; same locality, 28.VIII-16.IX.2013, $10^{7} 1$ ㅇ, leg. Negro; Morgex-La Salle: Natural Reserve of Marais, $900 \mathrm{~m}$, riparian forest, 14.VI-5.VII.2011, $30^{\circ}$, leg. Paschetta \& Giuliano; same locality, 18.VIII-16.IX.2011, 1 o", leg. Paschetta \& Giuliano; same locality, wet meadow, 14.VI-5.VII.2011, $40^{\prime \prime}$, leg. Paschetta, Giuliano; same locality, grove of reeds, 11-26. VI.2013, 1 o", leg. Negro; same locality, grove of reeds, 12-26.
VII.2013, 1 ơ, leg. Negro; Pont-Saint-Martin: Natural Reserve of Stagno di Holay, $616 \mathrm{~m}$, alpine grassland, 28.VIII-16.IX.2013, $10^{n}$, leg. Negro.

Literature. - Rossi \& Arnò 1995; [Isaia 2005]; [Rossi \& Bosio 2012].

Chorotype. - OLA.

MACROHABITAT. - Riparian habitats, alpine grasslands, wetlands.

Ozyptila pullata (Thorell, 1875)

NEW DATA. - Saint Denis: Brison-Cly, $1000 \mathrm{~m}$, broadleaved forest, 24.V-13.VI.2012, 1 ơ, leg. Negro (MRSN-VDA).

Literature. - Rossi \& Arnò 1995; [Isaia 2005]; [Rossi \& Bosio 2012].

Chorotype. - EUR.

MACROHABITAT. - Broadleaved forests, shrublands.

\section{Ozyptila rauda Simon, 1875}

NEW DATA. — Brissogne, Saint-Marcel, Quart, Nus: Natural Reserve of Les Îles, $530 \mathrm{~m}$, riparian vegetation, 18.VIII-16.IX.2011, $10^{7}$, leg. Paschetta \& Giuliano.

Literature. - Gobbi et al. 2010; [Rossi \& Bosio 2012].

CHOrotype. - SIE.

MACROHABITAT. - Rocky lands, riparian habitats.

\section{${ }^{*}$ Ozyptila secreta Thaler, 1987}

NEW DATA. - Saint Christophe: Tsatelet, $750 \mathrm{~m}$, broadleaved forest, 24.V-13.VI.2012, 1 o' 1 \% , leg. Negro (MRSN-VDA).

Chorotype. - ALPC.

MACROHABITAT. - Broadleaved forests.

Notes. - New record for Aosta Valley.

${ }^{*}$ Ozyptila simplex (O. P.-Cambridge, 1862)

NEW DATA. - Arvier: Lago di Lolair, 1175 m, wet meadow, 14.VI-5.VII.2011, $10^{7} 1 \%$, leg. Paschetta \& Giuliano; same locality, 18.VIII-16.IX.2011, 2 \%, leg. Paschetta \& Giuliano; Pont-Saint-Martin: Natural Reserve of Stagno di Holay, 775 m, wet meadow, 13.VI-4.VII.2011, 1 ơ , leg. Paschetta \& Giuliano; same locality, 13.VI-4.VII.2011, $10^{\prime \prime} 1$ \% , leg. Paschetta \& Giuliano (MRSN-VDA); same locality, riparian vegetation, 4-28. VII.2011, 1 ㅇ, leg. Paschetta \& Giuliano; Verrayes: stagno di Loson, 1520 m, grove of reeds/peat bog, 13.VI-4.VII.2011, $1 \mathrm{c}^{\text {", }}$ leg. Paschetta \& Giuliano.

CHorotype. - SIE.

MACROHABITAT. - Wetlands, riparian habitats.

Notes. - New record for Aosta Valley. 


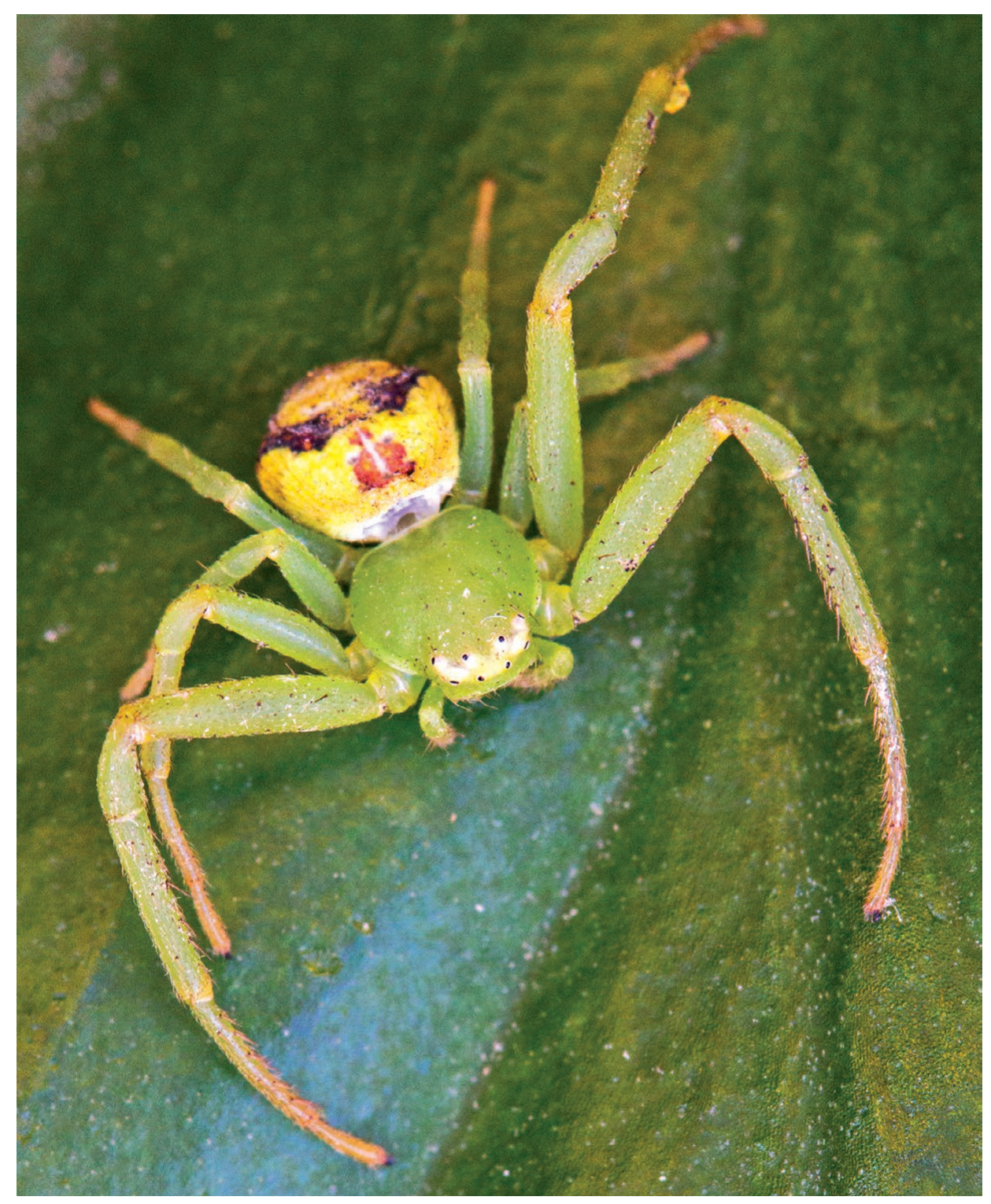

FIG. 15. - Ebrechtella tricuspidata (Fabricius, 1775) (Thomisidae), female. Average body length of the female: 6 mm. Natural Reserve of Les îles de Saint Marcel. Photograph: Mauro Paschetta 2011.

Ozyptila trux (Blackwall, 1846)

NEW DATA. — Torgnon: Lo Ditor, 1950 m, peat bog, 12-26.VII.2013, 1 \%, leg. Negro; same locality, 26.VII-14.VIII.2013, 2 ơ, leg. Negro; same locality, 14-28.VIII.2013, 1 o", leg. Negro.

Literature. - Negro et al. 2009, 2013; [Rossi \& Bosio 2012].

Chorotype. - PAL.

MacrohabitaT. - Wetlands, ski pistes.

•Runcinia grammica (C. L. Koch, 1837)

Literature. — Pesarini 1997; [Isaia 2005]; [Rossi \& Bosio 2012].

CHOROTYPe. - SCO.
MacrohabitaT. - Not specified.

Notes. - See notes about Hypsosinga heri.

*Synema globosum (Fabricius, 1775)

NEW DATA. — La Thuile: Comba Thuilette, 2000 m, wet meadow, 16.VIII.2011, 1 \%, leg. Paschetta \& Giuliano (MRSN-VDA); Challand-Saint-Victor: Natural Reserve of Lago di Villa, 820 m, wet meadow, 14.IX.2012, 1 \%, leg. Paschetta \& Mammola.

Chorotype. - PAL.

Macrohabitat. - Wetlands.

Notes. - New record for Aosta Valley. 


\section{Thomisus onustus Walckenaer, 1805} (Fig. 16)

LiterATURE. - Rossi \& Arnò 1995; [Isaia 2005]; [Rossi \& Bosio 2012].

Chorotype. - PAL.

Macrohabitat. - Shrublands.

Tmarus piger (Walckenaer, 1802)

Literature. - Rossi \& Arnò 1995; [Isaia 2005]; [Rossi \& Bosio 2012].

Chorotype. - PAL.

Macrohabitat. - Shrublands.

Xysticus audax (Schrank, 1803)

NEW DATA. - Courmayeur: Val Ferret, 1700 m, peat bog, 11.VII.2012, 1 ơ, leg. Paschetta, Giuliano \& Mammola; Torgnon: Lo Ditor, 1950 m, peat bog, 26.VI-12.VII.2013, 2 o", leg. Negro; same locality, 26.VII-14.VIII.2013, 1 \%, leg. Negro; Ayas: Antagnod, 1970-2070 m, alpine pasture, 2007, leg. Franco \& Negro.

LiterATURE. - Di Caporiacco 1928; [Isaia 2005]; De Angelis \& Fantoni 2008; Negro et al. 2009; [Rossi \& Bosio 2012].

Chorotype. - PAL.

MACROHABITAT. - Alpine pastures, wetlands.

\section{Xysticus bifasciatus C. L. Koch, 1837}

NEW DATA. - Torgnon: Lo Ditor, 1950 m, peat bog, 26.VI-12. VII.2013, 1 ơ $^{\prime}$, leg. Negro (MRSN-VDA); Ayas: Antagnod, 19702070 m, 2007, leg. Franco \& Negro.

Literature. - Calloni 1889; [Isaia 2005]; Negro et al. 2009; [Rossi \& Bosio 2012].

Chorotype. - PAL.

MACROHABITAT. - Wetlands, alpine prairies.

\section{-Xysticus bliteus (Simon, 1875)}

Literature. — Rossi \& Bosio 2012.

Chorotype. - MED.

MACROHABITAT. - Not specified.

Notes. - See Tegenaria domestica.

-Xysticus cor Canestrini, 1873

Literature. — Di Caporiacco 1928; [Isaia 2005].

Chorotype. - SEU.

MacrohabitaT. - Not specified.
NOTES. - Old record in need of validation. The occurrence of this species in Aosta Valley is aslo considered doubtful by Rossi \& Bosio (2012).

\section{Xysticus cristatus (Clerck, 1757)}

NEW DATA. - Ayas: Antagnod, 1970-2070 m, alpine pasture, 2007, leg. Franco \& Negro; Ayas, Antagnod, 1970-2070 m, ski pistes, 2007, leg. Franco \& Negro; Sarre-Saint Pierre: Monte Torretta, $900 \mathrm{~m}$, broadleaved forest, 25.VII-20.VIII.2012, 1 ㅇ, leg. Negro.

Literature. - Di Caporiacco 1928; [Isaia 2005]; De Angelis \& Fantoni 2008; Gobbi et al. 2010; [Rossi \& Bosio 2012].

Chorotype. - PAL.

Macrohabitat. - Alpine pastures, ski pistes, coniferous forests, broadleaved forests.

\section{Xysticus desidiosus Simon, 1875}

Literature. - Di Caporiacco 1928; [Isaia 2005]; Negro et al. 2010, 2013; [Rossi \& Bosio 2012].

CHOROTYPe. - SEU.

Macrohabitat. - Alpine prairies, ski pistes.

\section{Xysticus erraticus (Blackwall, 1834)}

NEW DATA. - Challand-Saint-Victor: Natural Reserve of Lago di Villa, 820 m, wet meadow, 11-26.VI.2013, 1 ㅇ, leg. Negro; Saint Christophe: Tsatelet, $750 \mathrm{~m}$, broadleaved forest, 13.VI-4.VII.2012, $10^{7}$, leg. Negro (MRSN-VDA); Pont-Saint-Martin: Natural Reserve of Stagno di Holay, 616 m, meadow, 26.VI-12.VII.2013, 10 , leg. Negro.

Literature. - Di Caporiacco 1928; [Isaia 2005]; De Angelis \& Fantoni 2008; Negro et al. 2009; [Rossi \& Bosio 2012].

Chorotype. - EUR.

MACROHABITAT. - Alpine grasslands, broadleaved forests, wetlands.

\section{Xysticus gallicus Simon, 1875}

NeW DATA. - Torgnon: Lo Ditor, 1950 m, peat bog, 26.VI-12. VII.2013, 1 o $^{\prime}$, leg. Negro (MRSN-VDA); Torgnon: Lo Ditor, 1950 m, peat bog, 26.VII-14.VIII.2013, 2 ơ 1 ㅇ, leg. Negro; Ayas: Antagnod, 1970-2070 m, ski pistes, 2007, leg. Franco \& Negro; same locality, alpine pasture, 2007, leg. Franco \& Negro.

Literature. - Di Caporiacco 1928; De Angelis \& Fantoni 2008; Negro et al. 2009, 2013 [Rossi \& Bosio 2012].

CHOROTYPe. - SIE.

MaCrOHABITAT. - Wetlands, alpine pastures, ski pistes. 


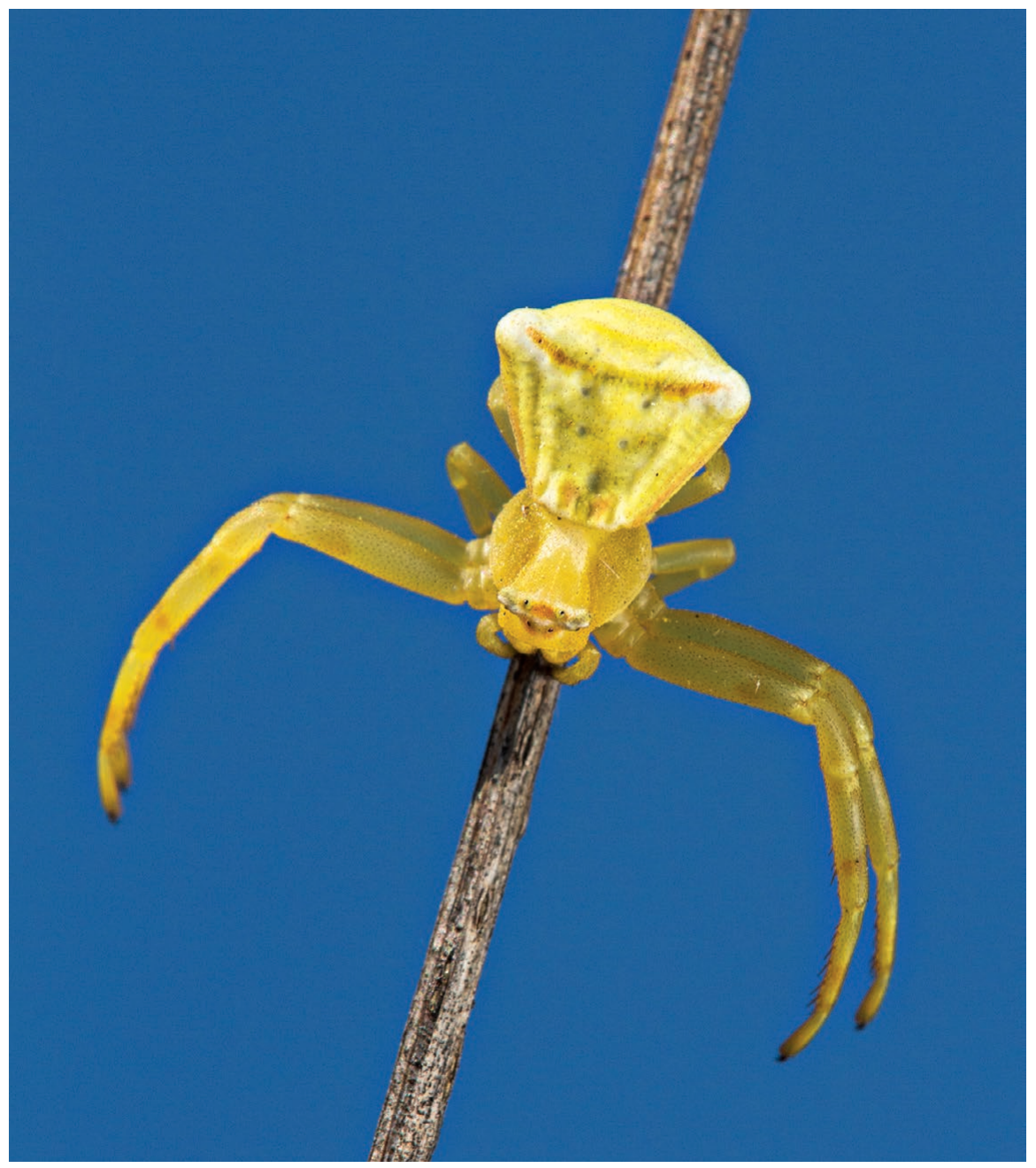

FIG. 16. - Thomisus onustus Walckenaer, 1805 (Thomisidae), female. Average body length of the female: 7-10 mm. Natural Reserve of Les Îles de Saint-Marcel. Photograph: Mauro Paschetta 2012.

\section{Xysticus kochi Thorell, 1872}

NEW DATA. - Sarre-Saint Pierre: Monte Torretta, $900 \mathrm{~m}$, dry meadow, 3-24.V.2012, 2 \%, leg. Negro; Brissogne, Saint-Marcel, Quart, Nus: Natural Reserve of Les Iles, 526 m, dry meadow, 11 26.VI.2013, 2 \%, leg. Negro; Pont-Saint-Martin: Natural Reserve of Stagno di Holay, 616 m, meadow, 11-26.VI.2013, 1 ơ, leg. Negro (MRSN-VDA).

Literature. - Di Caporiacco 1928; [Isaia 2005]; De Angelis \& Fantoni 2008; Negro et al. 2009; [Rossi \& Bosio 2012].

Chorotype. - SIE.

MACROHABITAT. - Alpine grasslands, shrublands.

\section{Xysticus lanio C. L. Koch, 1835}

Literature. - De Angelis \& Fantoni 2008; Gobbi et al. 2010; [Rossi \& Bosio 2012].

Chorotype. - SIE.

MacrohabitaT. - Coniferous forests, rocky lands.

\section{Xysticus ninnii Thorell, 1872}

NeW DATA. - Aymavilles: Pont d'Ael, $800 \mathrm{~m}$, broadleaved forest, 13.VI-4.VII.2012, $10^{7}$, leg. Negro; same locality, 13.VI-4. VII.2012, 1 \%, leg. Negro (MNHN); same locality, dry meadow, 13.VI-4.VII.2012, 1 ơ, leg. Negro; same locality, 4-25.VII.2012, 1 \%, leg. Negro (MRSN-VDA); same locality, 4-25.VII.2012, 1 o", $^{\prime \prime}$ leg. Negro; Saint Denis: Brison-Cly, $1000 \mathrm{~m}$, broadleaved forest, 4-25.VII.2012, 3 o", leg. Negro; same locality, 4-25.VII.2012, $10^{\text {", }}$ leg. Negro (MRSN-VDA); Sarre-Saint Pierre: Monte Torretta, 900 m, dry meadow, 13.VI-4.VII.2012, 3 o", leg. Negro; Challand-SaintVictor: Natural Reserve of Lago di Villa, 820 m, meadow, 26.VI12.VII.2013, 2 o", leg. Negro; Ayas: Antagnod, 1970-2070 m, ski pistes, 2007, leg. Franco \& Negro; same locality, alpine pasture, 2007, leg. Franco \& Negro.

Literature. - Di Caporiacco 1928; [Isaia 2005]; De Angelis \& Fantoni 2008; Negro et al. 2009; [Rossi \& Bosio 2012].

CHOROTYPe. - SIE.

MACROHABITAT. - Broadleaved forests, coniferous forests, alpine grasslands, alpine pastures, ski pistes, shrublands. 


\section{•Xysticus nubilus Simon, 1875}

Literature. - Di Caporiacco 1928; [Isaia 2005]; [Rossi \& Bosio 2012].

Chorotype. - MED.

MACROHABITAT. - Not specified.

NoTes. - Old record in need of validation.

\section{Xysticus robustus (Hahn, 1832)}

NEW DATA. - Saint Christophe: Tsatelet, $750 \mathrm{~m}$, broadleaved forest, 4-25.VII.2012, 1 o", leg. Negro; same locality, 13.VI-4.VII.2012, 10 , leg. Negro (MRSN-VDA); Brissogne, Saint-Marcel, Quart, Nus: Natural Reserve of Les Îles, 526 m, dry meadow, 26.VII-14. VIII.2013, 1 ơ, leg. Negro; Pont-Saint-Martin: Natural Reserve of Stagno di Holay, 616 m, meadow, 12-26.VII.2013, $10^{\text {" }} 1$ \%, leg. Negro; Challand-Saint-Victor: Natural Reserve of Lago di Villa, 820 m, meadow, 26.VII-14.VIII.2013, 1 ㅇ, leg. Negro.

Literature. - Di Caporiacco 1928; [Isaia 2005]; De Angelis \& Fantoni 2008; [Rossi \& Bosio 2012].

Chorotype. - CAE.

MACROHABITAT. - Broadleaved forests, alpine grasslands, shrublands.

Family Titanoecidae Lehtinen, 1967

-Titanoeca nivalis Simon, 1874

LiTERATURE. - Calloni 1889.

Chorotype. - OLA.

MACROHABITAT. - Not specified.

Notes. - Old record in need of validation.

Family Trachelidae Simon, 1897

Cetonana laticeps (Canestrini, 1868)

NEW DATA. - Challand-Saint-Victor: Natural Reserve of Lago di Villa, $820 \mathrm{~m}$, broadleaved forest, 26.VI-12.VII.2013, 1 \%, leg. Negro; same locality, wet meadow, 14.IX.2012, 1 \%, leg. Paschetta \& Mammola.

Literature. - De Angelis \& Fantoni 2008; [Rossi \& Bosio 2012].

CHOrotype. - EUM.

MACROHABITAT. - Broadleaved forests, wetlands.

Family UlObORIDAE Thorell, 1869

Uloborus plumipes Lucas, 1846

Literature. — Rossi \& Bosio 2014.
Chorotype. - MED.

MaCrohabitaT. - Synanthropic habitats.

•Uloborus walckenaerius Latreille, 1806

Literature. - Rossi \& Bosio 2012.

Chorotype. - PAL.

MACROHABITAT. - Synanthropic habitats.

Notes. - See Tegenaria domestica.

Family ZODARIIDAE Thorell, 1881

Zodarion italicum (Canestrini, 1868)

NEW DATA. - Challand-Saint-Victor: Natural Reserve of Lago di Villa, 820 m, wet meadow, 13.VI-4.VII.2011, 5 o 4 9 , leg. Paschetta \& Giuliano; same locality, 4-28.VII.2011, 2 \%, leg. Paschetta \& Giuliano, MNHN; same locality, 11-26.VI.2013, 15 ơ 10 ९, leg. Negro; same locality, 26.VII-14.VIII.2013, 4 o , leg. Negro; same locality, 14-28. VIII.2013, $10^{7}$, leg. Negro; same locality, broadleaved forest, 26.VII14.VIII.2013, 1 \% , leg. Negro; same locality, 11-26.VI.2013, 1 \%, leg. Negro; Saint Christophe: Tsatelet, $750 \mathrm{~m}$, broadleaved forest, 24.V-13.VI.2012, 1 o", leg. Negro; same locality, 4-25.VII.2012, 1 , leg. Negro (MRSN-VDA); Pont-Saint-Martin: Natural Reserve of Stagno di Holay, $702 \mathrm{~m}$, broadleaved forest (Castanea sativa), 11 26.VI.2013, 5 o' 1 \%, leg. Negro; Aymavilles: Pont d'Ael, 800 m, dry meadow, 3-24.V.2012, 1 0", leg. Negro.

Literature. - Isaia 2005; De Angelis \& Fantoni 2008; [Rossi \& Bosio 2012].

Chorotype. - EUR.

MACROHABITAT. - Broadleaved forests, wetlands, shrublands, alpine grasslands.

\section{Zodarion rubidum Simon, 1914}

NEW DATA. - Brissogne, Saint-Marcel, Quart, Nus: Natural Reserve of Les Îles, $530 \mathrm{~m}$, dry meadow, 14.VI-5.VII.2011, $60^{7} 5$ \% , leg. Paschetta \& Giuliano; same locality, 5-29.VII.2011, $10^{7} 1$ \%, leg. Paschetta \& Giuliano; same locality, 11-26.VI.2013, $10^{7} 2$ 웅, leg. Negro; Aymavilles: Pont d'Ael, 800 m, dry meadow, 3-24.V.2012, 1 o", leg. Negro; same locality, 24.V-13.VI.2012, 9 ơ 1 ㅇ, leg. Negro; same locality, 13.VI-4.VII.2012, $1 \mathrm{O}^{\text {" }} 4$ \% , leg. Negro; same locality, 4-25.VII.2012, 1 \%, leg. Negro; same locality, broadleaved forest, 13.VI-4.VII.2012, 7 ơ 2 ㅇ, leg. Negro; Morgex-La Salle: Natural Reserve of Marais, 900 m, riparian forest, 14.VI-5.VII.2011, $20^{7}$, leg. Paschetta \& Giuliano; same locality, wet meadow, 14.VI-5. VII.2011, 2 \%", leg. Paschetta \& Giuliano; same locality, grove of reeds, 26.VI-12.VII.2013, 3 ơ", leg. Negro; same locality, 26.VII-14. VIII.2013, 1 9, leg. Negro (MRSN-VDA); Saint Denis: Brison-Cly, 1000 m, dry meadow, 13.VI-4.VII.2012, 2 ơ, leg. Negro; same locality, broadleaved forest, 24.V-13.VI.2012, 2 ơ 1 \% , leg. Negro; Saint Denis: Brison-Cly, 1000 m, broadleaved forest, 13.VI-4.VII.2012, $1 \%$, leg. Negro; same locality, 4-25.VII.2012, 2 \%, leg. Negro; Gressan: Natural Reserve of Côte de Gargantua, $700 \mathrm{~m}$, broadleaved forest, 13.VI-4.VII.2012, 1 \%, leg. Negro, same locality, dry meadow, 
TABLE 3. - Number of families and species of spiders in some Northern Italian regions (NW Italy) and some cantons of Switzerland.

\begin{tabular}{|c|c|c|c|c|c|}
\hline Area/region & Families & Species & Extent $\left(\mathrm{km}^{2}\right)$ & Species/ km² & References \\
\hline Valle d'Aosta & 36 & 384 & 3.260 & 0.12 & This work \\
\hline Piemonte & 43 & 546 & 25.402 & 0.02 & Isaia et al. 2007 \\
\hline Lombardia & 39 & 679 & 23.860 & 0.02 & Isaia et al. 2007 \\
\hline Trentino - Alto Adige & 35 & 606 & 13.607 & 0.04 & Noflatscher 1996 \\
\hline Veneto & 44 & 738 & 18.399 & 0.04 & Ballarin et al. 2011 \\
\hline Italy & 55 & 1596 & 301.304 & 0.005 & Pantini \& Isaia 2015 \\
\hline Wallis & - & 512 & 5.224 & 0.09 & Maurer \& Hänggi 1990 \\
\hline Tessin & - & 510 & 2.812 & 0.18 & Maurer \& Hänggi 1990 \\
\hline
\end{tabular}

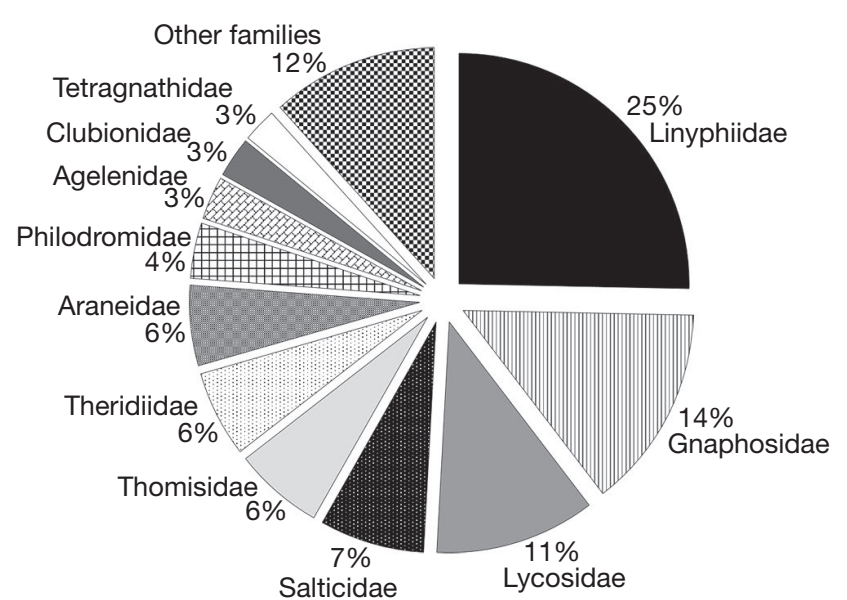

FIG. 17. - Species richness (in percentage) by family.

4-25.VII.2012, 2 ㅇ, leg. Negro; same locality, 24.V-13.VI.2012, 1 운 leg. Negro; Arvier: Lago di Lolair, 1175 m, wet meadow, 14.VI-5. VII.2011, $10^{\top} 1$ ㅇ, leg. Paschetta \& Giuliano; same locality, broadleaved forest, 13.VI-4.VII.2012, 1 \%, leg. Negro; Sarre-Saint Pierre: Monte Torretta, 900 m, dry meadow, 24.V-13.VI.2012, $10^{7} 1$ \% leg. Negro; same locality, 13.VI-4.VII.2012, 1 ơ 3 \% , leg. Negro; same locality, 4-25.VII.2012, 1 \%, leg. Negro.

LiterATURE. — Rossi \& Arnò 1995; [Isaia 2005]; [Rossi \& Bosio 2012].

Chorotype. - EUR.

MACROHABITAT. — Shrublands, broadleaved forests, riparian habitats, wetlands.

\section{GENERAL CONSIDERATIONS}

The catalogue provides an overall view on the spider fauna of the Italian administrative region of Aosta Valley and represents a term of reference for other similar Alpine regions. Excluding doubtful and uncertain species, according to our work, Aosta Valley hosts in total 382 species, grouped in 36 families and 182 genera. Despite it appears plausible, the occurrence of sixty-seven additional species reported in previous works

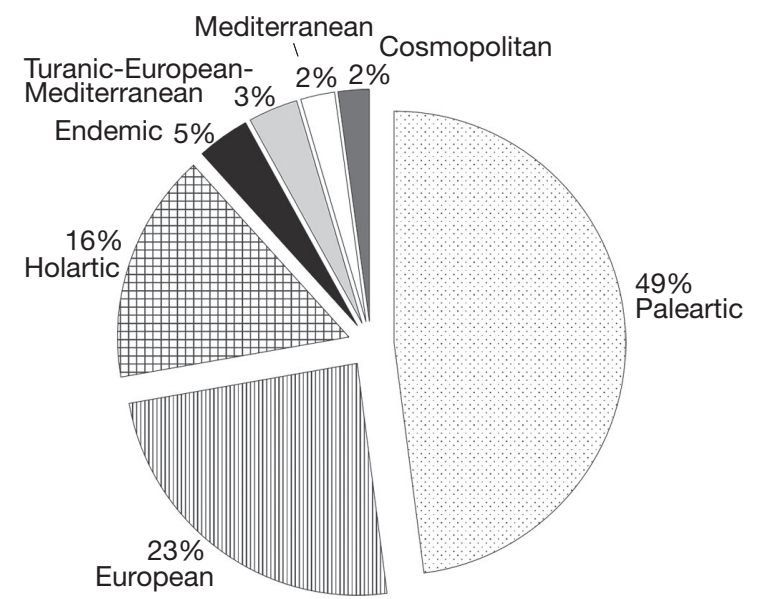

FIG. 18. - Species distribution. Groups of main chorotypes according to table 2.

is here regarded as doubtful, since it can not be verified directly or it has not been confirmed by our original data. On the other hand, the occurrence of seven species reported in literature is here questioned and considered uncertain on the base of taxonomical and faunistic arguments.

In terms of species richness, linyphiids dominate with 99 species (26\%), followed by gnaphosids (50 species, 13\%), lycosids (45 species, 11\%) and salticids (30 species, 7\%) (Fig. 17).

Compared with other northern Italian regions, such as Piedmont, Trentino-Alto Adige, Lombardy and Veneto, and considering its extent, Aosta Valley presents a relatively high number of species (see Table 3). When considering the Italian fauna (Pantini \& Isaia 2015), Aosta Valley hosts 25\% of the Italian species. Almost half the species (49\%) have a Palearctic distribution, nearly one-fourth (23\%) European and 16\% Holarctic (Table 2; Fig. 18). The same pattern of distribution is found in the nearby regions of Piedmont and Lombardy (respectively $47 \%$ and $47 \%$ of Palearctic species, $24 \%$ and $27 \%$ of European species and $16 \%$ and $14 \%$ of Holarctic species).

Despite difference may reflect different sampling efforts, in general terms, and in accordance with literature (Patocchi 1993; Villepoux 1993), wetlands host cumulatively the 


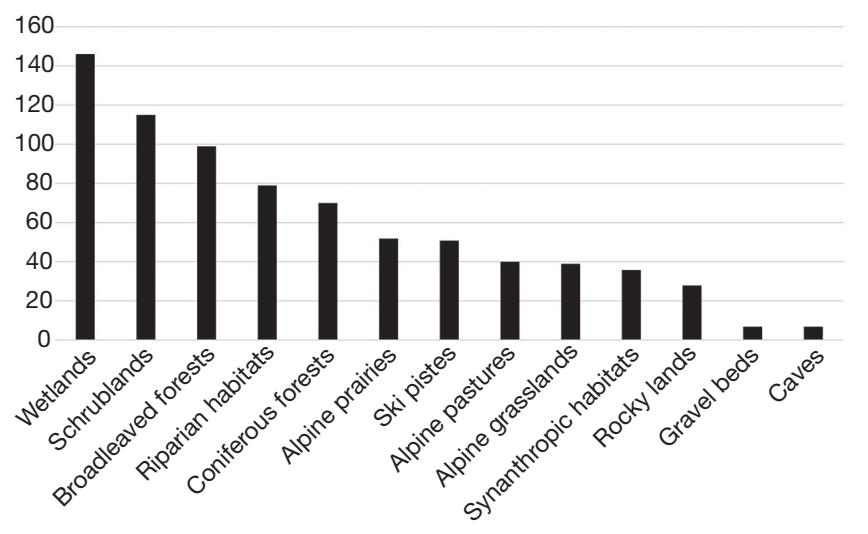

FIG. 19. - Number of species recorded in the considered macrohabitat.

highest number of spider species (146 species), followed by shrublands (115), broadleaved forests (99) and riparian habitats (79) (Fig. 19).

Wetlands and riparian habitats host the highest richness of linyphiids (respectively with 33 and 23 species) while diversity of gnaphosids and lycosids is higher in wetlands and shrublands (respectively 24 and 21 species of gnaphosids and 21 and 19 species of lycosids). Salticids diversity is higher in wetlands and shrublands as well as in rocky lands (respectively 10,10 and nine species).

In contrast to other Italian regions (possibly in relation to its smaller surface), Aosta valley does not have any species which are exclusively found within its territory. On the other hand, several species are distributed in small sectors of the Alpine chain encompassing parts of the region (Table 4) ("endemic or subendemc to geographic Italy" according to Vigna Taglianti et al. 1999). This group of species represents $5 \%$ of the spider fauna of Aosta Valley, a lower value compared to Piedmont (7\%) and Lombardy (6\%) (Isaia et al. 2007).

The original data presented here add 76 species to the list of the spider species previously known for the study area. Most of the new records for the study area are the result of an intense campaign of samplings in wetlands and xerothermic area of the Aosta valley during 2011 and 2013, which were previously scarcely investigated. Nevertheless, most of the region remain unexplored (Fig. 20). Including literature records, our work reports data from 168 localities, covering a relatively small part of the regional territory and mostly concentrated at mid-high elevation. Further research should specifically focus on the valley bottom, with a specific focus on agricultural areas (such as vineyards and other perennial cultivation), largely neglected in previous works, and potentially hosting a large number of unrecorded species.

Concerning species with restricted distribution, it is worth to mention Coelotes pickardi and C. rudolfi, whose ranges are centred in the Western Alps (Fig. 21). Among subterranean species, Troglohyphantes nigraerosae stands out as one of the rarest species, with a very restricted range bordering Pie-
TABLE 4. - List of Alpine endemic species (sensu Vigna Taglianti et al. 1999) found in the study area, with reference to the collection localities and to the macrohabitat (alphabetic order after World Spider Catalogue 2015).

\begin{tabular}{|c|c|}
\hline Species & Localities \\
\hline $\begin{array}{l}\text { Agyneta alpica } \\
\text { Tanasevitch, } 2000\end{array}$ & $\begin{array}{l}\text { Gressoney la Trinité: Passo dei Salati } \\
\text { Gressoney la Trinité: Passo di } \\
\text { Bettaforca }\end{array}$ \\
\hline $\begin{array}{l}\text { Arctosa renidescens } \\
\quad \text { Buchar \& Thaler, } 1995\end{array}$ & $\begin{array}{l}\text { La Thuile: Comba Thuilette } \\
\text { Ayas: Antagnod }\end{array}$ \\
\hline $\begin{array}{l}\text { Berlandina nubivaga } \\
\text { (Simon, 1878) }\end{array}$ & Torgnon \\
\hline $\begin{array}{l}\text { Coelotes pickardi } \\
\text { O. P.- Cambridge, } 1873\end{array}$ & $\begin{array}{l}\text { Valsavaranche: Orvieille } \\
\text { Cogne: Valnontey, Rifugio V. Sella, } \\
\text { Pondel } \\
\text { Gressoney la Trinité: Lago Gabiet, Passo } \\
\text { di Bettaforca, Passo dei Salati } \\
\text { Gressoney St. Jean } \\
\text { Ollomont } \\
\text { Champdepraz: Lac Gelè }\end{array}$ \\
\hline $\begin{array}{l}\text { Coelotes rudolfi (Schenkel, } \\
\text { 1925) }\end{array}$ & $\begin{array}{l}\text { Cogne: Valnontey, Rifugio V. Sella } \\
\text { Gressoney la Trinité: Passo di } \\
\text { Bettaforca }\end{array}$ \\
\hline $\begin{array}{l}\text { Cybaeus intermedius } \\
\text { Maurer, } 1992\end{array}$ & $\begin{array}{l}\text { Gressoney St. Jean } \\
\text { Pont-Saint-Martin: Natural Reserve of } \\
\text { Stagno di Holay }\end{array}$ \\
\hline
\end{tabular}

Haplodrassus aenus Thaler, Ayas: Antagnod $1984 \quad$ Torgnon

Harpactocrates drassoides Champdepraz: Natural Park of Mont (Simon, 1882) Avic

\begin{tabular}{cl} 
& Arvier: Lolair \\
\hline $\begin{array}{c}\text { Mansuphantes } \\
\text { pseudoarciger } \\
\text { (Wunderlich, 1985) }\end{array}$ & $\begin{array}{l}\text { Torgnon } \\
\text { Gressoney St. Jean }\end{array}$ \\
\hline $\begin{array}{c}\text { Mughiphantes armatus } \\
\text { (Kulczyński, 1905) }\end{array}$ & Ayas: Antagnod \\
\hline
\end{tabular}

Mughiphantes pulcheroides Aosta (Wunderlich, 1985)

\begin{tabular}{|c|c|}
\hline $\begin{array}{l}\text { Histopona leonardoi } \\
\text { Bolzern, Pantini \& Isaia, } \\
2013\end{array}$ & $\begin{array}{l}\text { Champdepraz: Natural Park of Mont Avic } \\
\text { Gressoney St. Jean } \\
\text { Gressoney la Trinité: Passo dei Salati } \\
\text { Ayas } \\
\text { Torgnon: Lo Ditor }\end{array}$ \\
\hline $\begin{array}{l}\text { Tapinocyba maureri } \\
\text { Thaler, } 1991\end{array}$ & Verrès \\
\hline $\begin{array}{l}\text { Troglohyphantes lucifuga } \\
\text { (Simon, 1884) }\end{array}$ & $\begin{array}{l}\text { St. Pierre: Piccola grotta, Combelin } \\
\text { La Salle: Grotta ghiacciata di } \\
\text { Chabaudey } \\
\text { Valsavaranche: Borna du Ran } \\
\text { Brusson: Trou de Rompailly } \\
\text { St.Pierre: Fessura di Verrogne } \\
\text { Courmayeur: Trou des Romains } \\
\text { Gressoney la Trinité: Grotta JO } 6 \text { di } \\
\text { Punta Jolanda, Grotta JO-12 Punta } \\
\text { jolanda } \\
\text { Sarre: Mezz'Abisso, Grotta della } \\
\text { Soldanella }\end{array}$ \\
\hline $\begin{array}{l}\text { Troglohyphantes } \\
\text { nigraerosae Brignoli, } \\
1971\end{array}$ & Cogne: Colle dell'Arietta \\
\hline $\begin{array}{l}\text { Zelotes devotus Grimm, } \\
1982\end{array}$ & $\begin{array}{l}\text { Courmayeur: Miage Glacier } \\
\text { Gressoney la Trinité }\end{array}$ \\
\hline
\end{tabular}




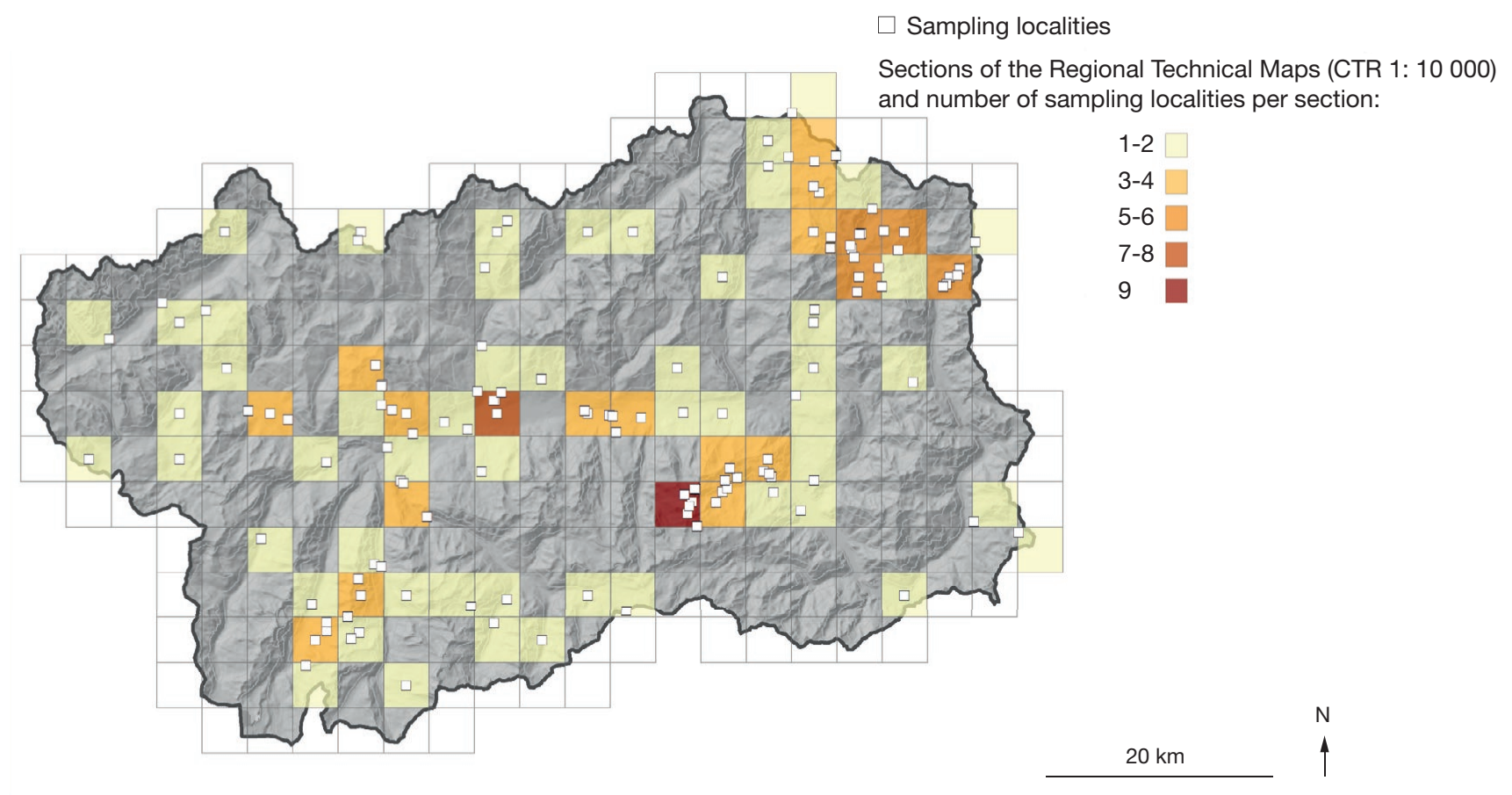

FIG. 20. - Map of the sampling localities (new data and literature).

monte and Aosta Valley. Literature records of this species are limited to the type locality (Colle dell'Arietta, marking the border between Piedmont and Aosta Valley) and a few more subterranean localities in Piedmont (Isaia et al. 2011). The subterranean domain in Aosta Valley is frequently inhabited by T. lucifuga (Simon, 1884) (Linyphiidae) (Figs 7; 22), being known for several caves in the region (Arnò \& Lana 2005; Isaia et al. 2010) with a distribution extending from Susa Valley (Piedmont) up to Switzerland.

Some species stand out for their ecological peculiarities, like Pardosa nigra (C. L. Koch, 1834) (Fig. 23), a big lycosid (body length: 7-9 mm), typical of block fields, rocky prairies and glaciers, generally faound at high elevations over $2000 \mathrm{~m}$ (Nentwig et al. 2015) and Sitticus longipes (Canestrini, 1873) a salticids with European distribution with similar ecological requirements (Fig. 24). Both species were found in several localities in Aosta Valley, always above $1700 \mathrm{~m}$.

Collections performed in xerothermic areas provided data on several new records for the region such as Ozyptila secreta Thaler, 1987 a rare species with a restricted Alpine distribution (Italy and Switzerland), typical of rocky heathland and xerothermic localities (Fig. 24) and Mesiotelus tenuissimus (L. Koch, 1866) (Liocranidae) a Mediterranean species found in the boadleaved forest of the Natural areas of Monte Torretta and Pont d'Ael. Another interesting xerophile species, Atypus muralis Bertkau, 1890 (Atypidae), a new record for the region, was found in the xerothermic area of Aymavilles (Pont d'Ael). Together with the congeneric $A$. affinis Eichwald,
1830 , these are the only two species of mygalomorph spiders known for the region.

Samplings in wetlands pointed out an array of interesting species, most of them being new records for the region. For example, Pirata piraticus (Clerck, 1757), P. tenuitarsis Simon, 1876 and Piratula hygrophila (Thorell, 1872) (Lycosidae) and Mendoza canestrinii (Ninni, 1868) (Salticidae) (Fig. 10) stand out for their strict dependence on wetland ecosystems.

Knowledge of local biodiversity is a key factor for nature conservation and management, and recapitulatory works, such as reliable species checklists, may specifically contribute to help stakeholders in the management of natural resources. In this work we underline the high diversity of the spider fauna of Aosta Valley and point out the importance of wetlands and xerothermic areas as important habitats for the conservation and the enhancement of biodiversity. Concerning previous data published so far in arachnological literature, we emphasize the low number of investigated localities in Aosta Valley, as well as a general lack of arachnological records from potential species-rich habitats, such as vineyards and other perennial agroecosystems, which characterize the landscape of the Aosta Valley's lowlands.

\section{Acknowledgements}

The authors would like to thank the two anonymous referees for providing important suggestions during revision. 

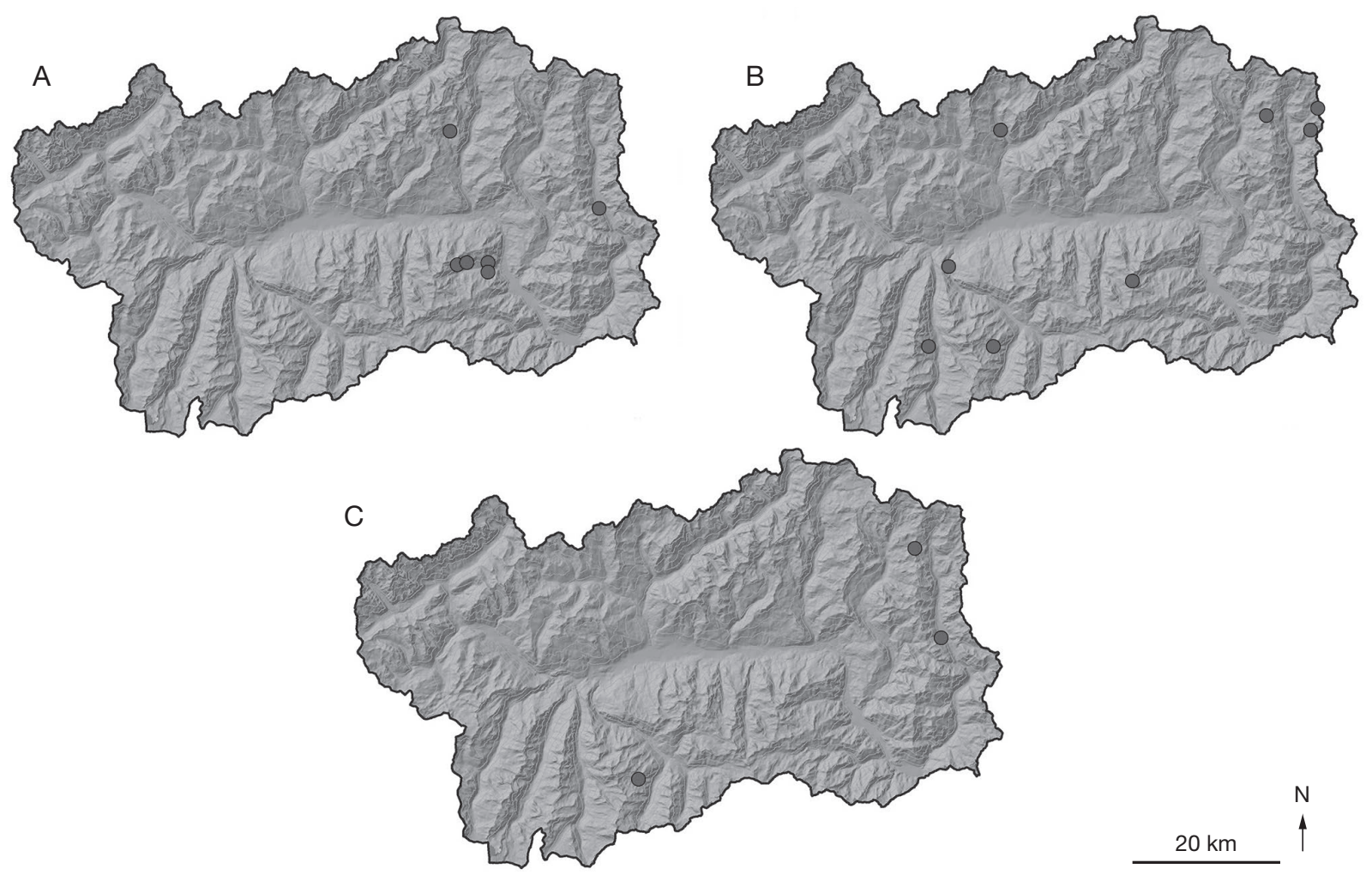

FIG. 21. - Records of species of faunistic or ecological interest: A, Coelotes mediocris Kulczyński, 1887; B, C. pickardi O. P.-Cambridge, 1873; C, C. rudolfi (Schenkel, 1925).
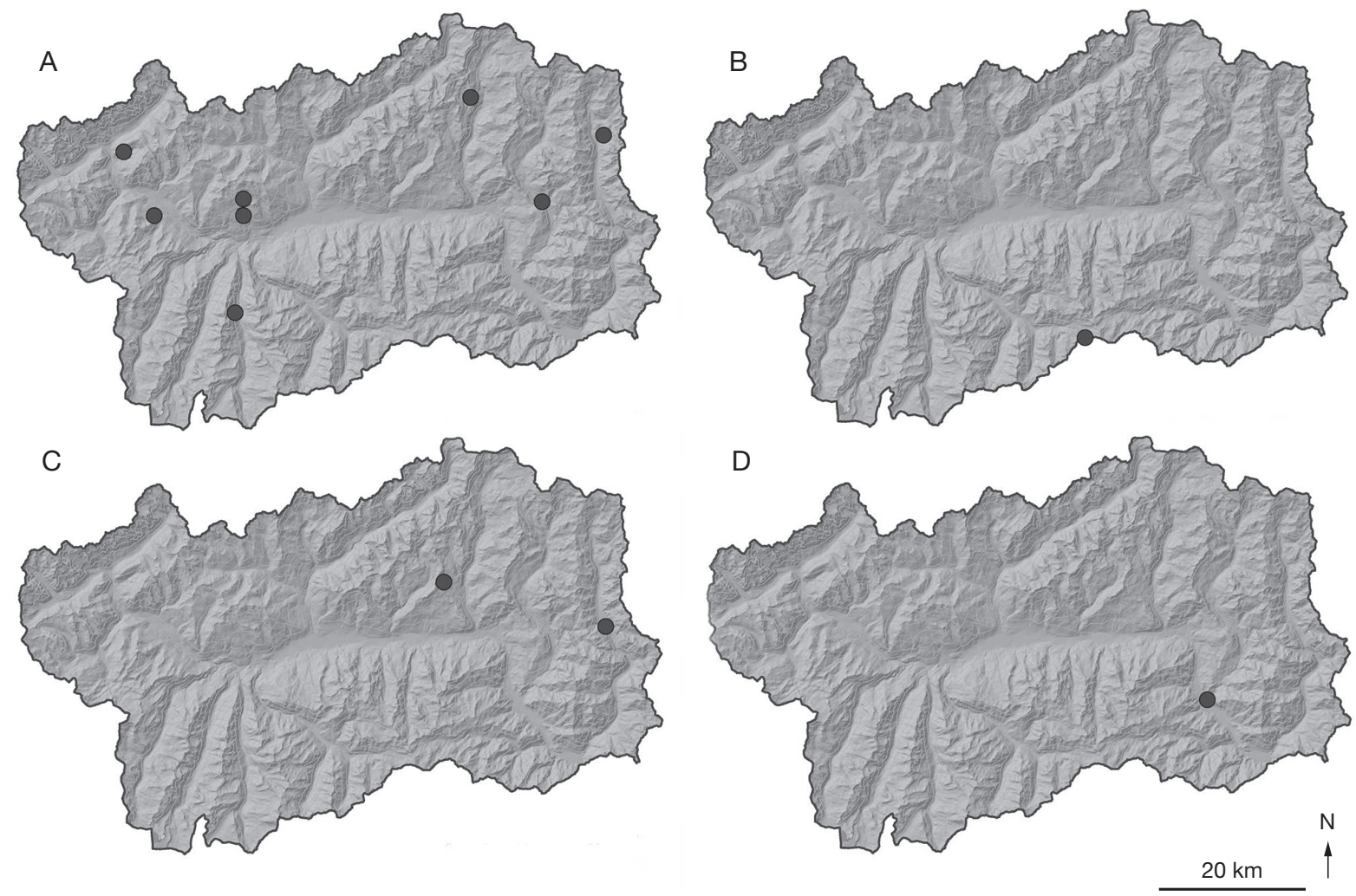

FIG. 22. - Records of species of faunistic or ecological interest: A, Troglohyphantes lucifuga (Simon, 1884); B, T. nigraerosae Brignoli, 1971; C, Mansuphantes pseudoarciger (Wunderlich, 1985); D, Tapinocyba maureri Thaler, 1991. 

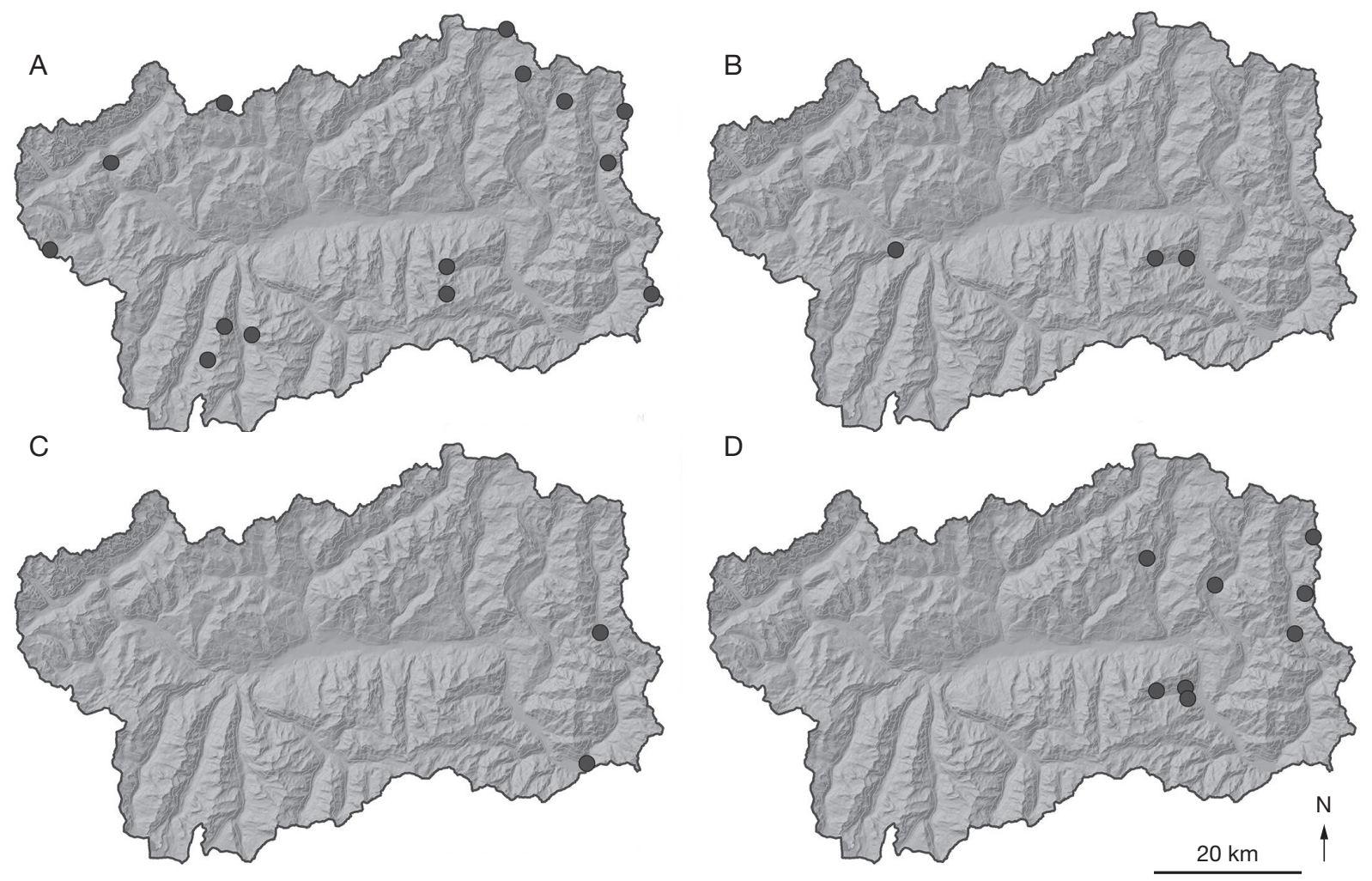

FIG. 23. - Records of species of faunistic or ecological interest: A, Pardosa nigra (C. L. Koch, 1834); B, Harpactocrates drassoides (Simon, 1882); C, Cybaeus intermedius Maurer, 1992; D, Histopona leonardoi Bolzern, Pantini \& Isaia, 2013.
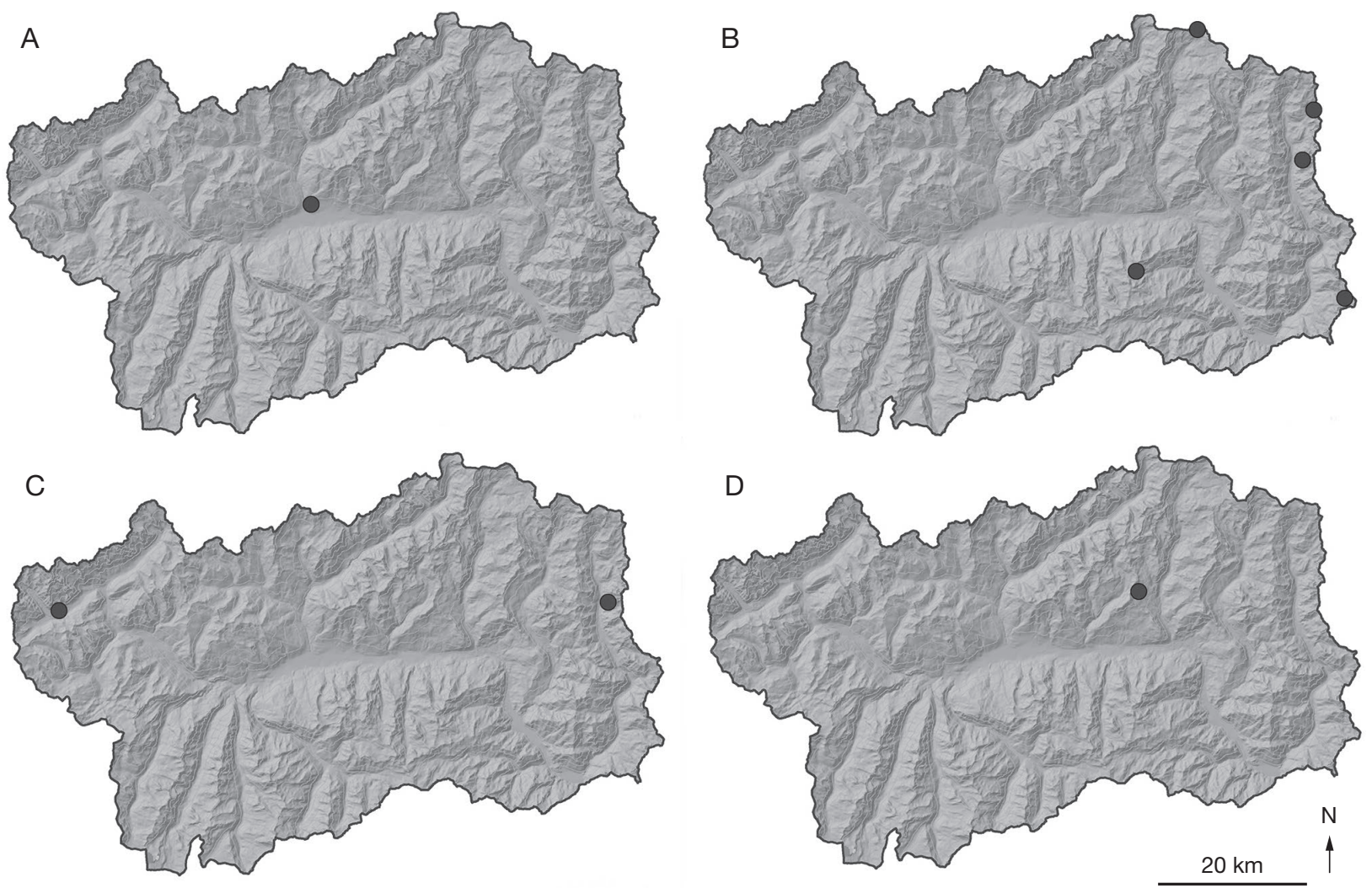

FIG. 24. - Records of species of faunistic or ecological interest: A, Ozyptila secreta Thaler, 1987; B, Sitticus longipes (L. Koch, 1866); C, Zelotes devotus Grimm, 1982; D, Berlandina nubivaga (Simon, 1878). 


\section{REFERENCES}

ARnÒ C. \& LANA E. 2005. - Ragni cavernicoli del Piemonte e della Valle d'Aosta. Associazione Gruppi Speleologici Piemontesi, Regione Piemonte, Torino, La Grafica Nuova, 265 p.

Ballarin F., PANTini P. \& Hansen H. 2011. - Catalogo ragionato dei ragni (Arachnida, Araneae) del Veneto. Museo Civico di Storia Naturale di Verona. 2 serie. Sezione Scienze della Vita 21: 151 p.

BocCa M. \& MAfFeI G. 1984. — Gli uccelli della Valle d'Aosta: indagine bibliografica e dati inediti. Tipografia la Vallée: Aosta (AO).

BolZern A. \& HängGi A. 2006. - Drassodes lapidosus und Drassodes cupreus (Araneae: Gnaphosidae)-eine unendliche Geschichte. Arachnologische Mitteilungen 31: 16-22

Bolzern A., Pantini P. \& Isaia M. 2013 - Revision of the Histopona italica group (Araneae: Agelenidae), with the description of two new species. Zootaxa 3640: 23-41.

Bovio M. 2014. — Flora vascolare della Valle d'Aosta: Repertorio commentato e stato delle conoscenze. Testolin: Sarre (AO).

Brignoli P. M. 1971a. — Note sui ragni cavernicoli italiani. Fragmenta Entomologica 7 (3): 121-229.

Brignoli P. M. 1971b. - Contributo alla conoscenza degli Agelenidae italiani. Fragmenta Entomologica 8: 57-142.

Brignoli P. M. 1977. — Ragni d'Italia XXVII. Nuovi dati su Agelenidae, Argyronetidae, Hahniidae, Oxyopidae e Pisauridae, cavernicoli ed epigei (Araneae). Quaderni del Museo di Speleologia "V. Rivera" L'Aquila 2 (4): 3-118.

BRIgNOLI P. M. 1979. - Ragni d'Italia XXXI. Specie cavernicole nuove o interessanti (Araneae). Quaderni del Museo Speleologico "V. Rivera" 5 (10): 1-48.

CALLONI S. 1889. - La fauna nivale con particolare riferimento ai viventi delle Alte Alpi, Pavia, 478 p. (estratto: 132-275).

Camerano P., Terzuolo P. \& VArese P. 2007. - I tipi forestali della Valle d'Aosta. Compagnia delle foreste: Arezzo.

De Angelis S. \& Fantoni A. 2007-2008. - Contributo alla conoscenza della fauna araneologica (Arachnida Araneae) del Parco Naturale del Monte Avic (Valle d'Aosta) con una nuova segnalazione per la fauna italiana. Revue Valdôtaine d'Histoire naturelle 61-62: 109-116.

De Blauwe R. 1975. - Deuxième contribution à l'étude des Agelenidae des collections du Musée d'Histoire naturelle de Vérone. Bollettino del Museo Civico di Storia Naturale di Verona 2: 145-154.

DI CAPORIACCO L. 1928. — Aracnidi. Il Parco Nazionale del Gran Paradiso, Torino 2: 1-4.

Fontana R., Cantarella T., Sala L. \& Tongiorgi P. 1996. — A collection of Italian Salticids (Araneae), mainly from the TuscanEmilian Apennines. Revue suisse de Zoologie (hors série): 211-222.

GobBi M., IsAia M. \& De BeRNARDI F. 2010. — Arthropod colonisation of a debris-covered glacier. The Holocene 21 (2): 343-349.

GRIMM U. 1985. — Die Gnaphosidae Mitteleuropas (Arachnida, Araneae). Abhandlungen des Naturwissenschaftlichen Vereins in Hamburg 26: 1-318.

HÄNGGI A. \& STÄUbli A. 2012. - Nachträge zum "Katalog der schweizerischen Spinnen" 4. Neunachweise von 2002 bis 2011. Arachnologische Mitteilungen 44: 59-76.

Hervé C., Roberts M. J., Murphy J. A. 2009. - A taxonomic revision of the genus Drassodex Murphy, 2007 (Araneae: Gnaphosidae). Zootaxa 2171: 1-28.

Hervé C. \& Rollard C. 2009. - Drassodes species from the Parc national du Mercantour (French Alps), with the description of a new species (Araneae: Gnaphosidae). Contributions to Natural History 12: 627-642.

ISAIA M. 2000. - Note su alcuni ragni (Arachnida Araneae) del Parco Naturale del Monte Avic (Valle d'Aosta) e sulla loro distribuzione altimetrica. Revue valdôtaine d'Histoire naturelle 54: 107-117.

IsAiA M. 2005. - Check list of spiders (Arachnids, Araneae) of Aosta Valley (Italy) with a new record for Italian fauna. Revue valdôtaine d'Histoire naturelle 59: 25-43.
ISAIA M. \& CHIARLE A. 2015. - Taxonomic notes on Cybaeus vignai Brignoli, 1977 (Araneae, Cybaeidae) and Dysdera cribrata Simon, 1882 (Araneae, Dysderidae) from the Italian Maritime Alps, in Daugeron C., Deharveng L., Isaia M., Villemant C. \& JudSON M. (eds), Mercantour/Alpi Marittime All Taxa Biodiversity Inventory. Zoosystema 37 (1): 45-56. http://dx.doi.org/10.5252/ z2015n1a3

IsAiA M. \& PANTINi P. 2009. - Coelotes pickardi O. Pickard-Cambridge, 1873: un intricato caso di sinonimia per un endemita alpino ritrovato in Valle Oropa e le sue sottospecie (Arachnida, Araneae, Amaurobiidae). Memorie dell'Associazione Naturalistica Piemontese 11: 23-28.

IsAiA M. \& PANTini P. 2010. - New data on the spider genus Troglohyphantes (Araneae, Linyphiidae) in the Italian Alps, with the description of a new species and a new synonymy. Zootaxa 2690: 1-18. http://dx.doi.org/10.11646/\%25x

Isaia M., Pantini P., Beikes S. \& Badino G. 2007. — Catalogo ragionato dei ragni (Arachnida, Araneae) del Piemonte e della Lombardia. Memorie dell'Associazione Naturalistica Piemontese 9: 1-161

Isaia M., Negro M. \& Rolando A. 2009. - Notes on the distribution of Berlandina nubivaga with the description of the male (Araneae, Gnaphosidae). Journal of Arachnology 36 (1): 180-183. http://dx.doi.org/10.1636/H07-26SC.1

Isaia M., Paschetta M., Lana E., Pantini P., Schönhofer A. L., Christian E. \& BADino G. 2011. — Aracnidi sotterranei delle Alpi Occidentali italiane/Subterranean Arachnids of the Western Italian. Torino, Museo Regionale di Scienze Naturali (Monografie 47$), 325 \mathrm{p}$.

LESSERT R. DE (1910). - Catalogue des invertébrés de la Suisse. Fasc. 3, Araignées. Musée d'histoire naturelle de Genève, 635 p.

Locket G. H. \& Millidge A. F. 1953. — British spiders, vol. 1. London, Ray Society, 449 p.

LugetTi G. \& Tongiongi P. 1969. - Ricerche sul genere Alopecosa Simon (Araneae - Lycosidae). Atti Società Toscana di Scienze Naturali, Memorie, Serie B 76: 1-100.

MaraZZI S. 2005. - Atlante Orografico delle Alpi. SOIUSA, Pavone Canavese, Priuli \& Verlucca.

Maurer R. 1982. - Zur Kenntnis der Gattung Coelotes (Araneae, Agelenidae) in Alpenländern II. Die pastor-Gruppe der italienischen und französischen Alpen - Beschreibung von C. pastor lessinensis ssp. n. Bollettino del Museo Civico di Storia Naturale di Verona 8: 165-183.

Maurer R. \& HängGi A. 1990. - Katalog der schweizerischen Spinnen. Documenta faunistica Helvetiae 12, 420 p.

Muster C. \& BerEndonK T. U. 2006. - Divergence and diversity: lessons from an arctic-alpine distribution (Pardosa saltuaria group, Lycosidae). Molecular Ecology 15 (10): 2921-2933.

Negro M., Isaia M., Palestrini C. \& Rolando A. 2009. The impact of forest ski-pistes on diversity of ground-dwelling arthropods and small mammals in the Alps. Biodiversity and Conservation 18 (11): 2799-2821. http://dx.doi.org/10.1007/ s10531-009-9608-4

Negro M., Isaia M., Palestrini C., Schoenhofer A. \& ROLANDO A. 2010. - The impact of high-altitude ski pistes on ground-dwelling arthropods in the Alps. Biodiversity and Conservation 19 (7): 1853-1870. http://dx.doi.org/10.1007/ s10531-010-9808-y

Negro M., Rolando A., Barni E., Bocola D., Filippa G., Freppaz M., Isaia M., SinisCAlco C. \& Palestrini C. 2013. — Differential responses of ground dwelling arthropods to ski-piste restoration by hydroseeding. Biodiversity and Conservation 22 (11): 2607-2634. http://dx.doi.org/10.1007/s10531-013-0544-y

Nentwig W, Blick T, Gloor D, HÄNGgi A \& Kropf C. 2015. Spiders of Europe. http://www.araneae.unibe.ch (last access, 26 feb. 2016).

NoflatsCher M.T. 1996. - Aranei - Spinnen, Webspinnen, in Hellrigl K. (ed.), Die Tierwelt Südtirols. Kommentiertes 
systematisch-faunistiches Verzeichnis der aut dem Gebiet der Provinz Bozen-Südtirol (Italien) bekannten Tierarten. Veröffentlichungen des Naturmuseums Südtirol, vol. 1, p. 211-228.

PANTINI P. \& IsAiA M. 2008. - New records for the Italian spider fauna (Arachnida, Araneae). Arthropoda Selecta 17 (1-2): 133-144.

PANTINI P. \& ISAIA M. 2015. — Checklist of Italian spiders. http:// www.museoscienzebergamo.it/web/index.php?option=com_con tent $\&$ view $=$ category $\&$ layout $=$ blog $\&$ id $=96 \&$ Itemid $=94$ (last access, 26 feb. 2016).

PAtocchi N. 1993. - I ragni della Valle Maggia: studio faunistico ecologico delle zone alluvionali. Memorie Società Ticinese di Scienze Naturali 3: 209-267.

PAVESI P. 1873. - Catalogo sistematico dei ragni del cantone ticino con la loro distribuzione orizontale e verticale e cenni sulla araneologica elvetica. Annali del Museo Civico di Storia Naturale di Genova 4: 5-215.

Pavesi P. 1904. - Esquisse d'une faune valdôtaine. Atti del Museo di Milano 43: 250-256.

PESARINI C. 1996. - Note su alcuni Erigonidae italiani, con descrizione di una nuova specie (Araneae). Atti della Società Italiana di Scienze Naturali e del Museo Civico di Storia Naturale di Milano 135 (II): 413-429.

PesARINI C. 1997. - I ragni (Arachnida Araneae) del Monte Barro (Italia, Lombardia, Lecco). Memorie della Società italiana di Scienze naturali, Museo civico di Storia naturale di Milano 27: 251-263.

PESARINI C. 2000. - Contributo alla conoscenza della fauna araneologica italiana (Araneae). Memorie della Società Entomologica Italiana 78: 378-393.

PESARINI C. 2001. - Note sui Trogolohyphantes italiani, con descrizione di quattro nuove specie (Araneae, Linyphiidae). Atti della Società italiana di Scienze naturali, Museo civico di Storia naturale di Milano 142 (1): 109-133.

ŘezÁč M., Gasparo F., Král J. \& Heneberg P. 2014. — Integrative taxonomy and evolutionary history of a newly revealed spider Dysdera ninnii complex (Araneae: Dysderidae). Zoological Journal of the Linnean Society 172: 451-474.

ROBERTS M. J. 1985. - The Spiders of Great Britain and Ireland, Volume 1: Atypidae to Theridiosomatidae. Harley Books, Colchester, England, 229 p.

RoBerTs M. J. 1995. - Collins Field Guide: Spiders of Britain \& Northern Europe. Harper Collins, London, 383 p.

Rossi G. \& ARNÒ C. 1995. — Indagini sull'araneofauna della zona umida della Natural Reserve of di Les Îles (Valle d'Aosta). Revue valdôtaine d'Histoire naturelle 49: 43-57.

Rossi G. \& Bosio F. 2012. — Elenco delle specie di ragni (Arachnida, Araneae) note per la Valle d 'Aosta. Revue valdôtaine d'Histoire naturelle 66: 45-88.
Rossi G. \& Bosio F. 2014. — Prima segnalazione per la Valle d'Aosta di Uloborus plumipes Lucas, 1846 (Arachnida, Araneae). Revue valdôtaine d'Histoire naturelle 68: 91-93.

SimON E. 1878. - Les arachnides de France, 4. Paris 4, Librarie encyclopédique de Roret, p. 1-334.

SimON E. 1881. - Les arachnides de France, 5. Paris, Librarie encyclopédique de Roret, p. 1-180.

SimON E. 1884. — Les arachnides de France, 5. Paris, Librarie encyclopédique de Roret, p. 180-885.

Stoch F. \& Vigna Taglianti A. 2005. - I corotipi della fauna italiana, in RUFFO S. \& STOCH F. (eds), Checklist e distribuzione della fauna italiana. Memorie del Museo Civico di Storia Naturale di Verona, 2 serie, Sezione Scienze della Vita 16: 25-28.

THALER K. 1982. - Weitere wenig bekannte Leptyphantes-Arten der Alpen (Arachnida: Aranei, Linyphiidae). Revue suisse de Zoologie 89: 395-417.

Töpfer-Hofmann G., Cordes D. \& Helversen O. von 2000. Cryptic species and behavioural isolation in the Pardosa lugubris group (Araneae, Lycosidae), with description of two new species. Bulletin of the British Arachnological Society 11: 257-274.

TONGIORGI P. 1966. - Italian wolf spiders of the genus Pardosa (Araneae, Lycosidae). Bulletin of The Museum of Comparative Zoology 134 (8): 275-334.

Vigna taglianti A., Audisio P. A., Belfiore C., Biondi M., Bologna M. A., Carpaneto G. M., De Biase A., de Felici S., PiatTElla E., RaChEli T., Zapparoli M. \& Zoia S. 1993. — Riflessioni di gruppo sui corotipi fondamentali della fauna W-Paleartica ed in particolare italiana. Biogeographia 16: 159-179.

Vigna Taglianti A., Audisio P. A., Biondi M., Bologna M. A., Carpaneto G. M., De Biase A., Fattorini S., Piattella E., Sindaco R., Venchi A. \& Zapparoli M. 1999. - A proposal for a chorotype classification of the Near East fauna, in the framework of the Western Palearctic region. Biogeographia 20: 31-59.

Villepoux O. 1993. — Étude de la répartition des araignées d'une zone humide. Proc. 14th Eur. Colloq. of Arachnology. Catania, Bollettino dell'Accademia Gioenia di Scienze Naturali 26: 361-370.

WOrLd SPIDER CATALOG (2015). — World Spider Catalog. Natural History Museum Bern, online at http://wsc.nmbe.ch, version 16, accessed on 26 feb. 2016.

WUNDERLICH J. 1984. — Seltene und bisher unbekannte Wolfspinnen aus Mitteleuropa und Revision der Pardosa saltuaria-Gruppe (Arachnida: Araneae: Lycosidae). Verhandlungen des naturwissenschaftlichen Vereins in Hamburg 27: 417-442.

WUNDERLICH J. 1985. - Lepthyphantes pulcheroides n. sp. aus Nord-Italien (Arachnida: Araneae: Linyphiidae). Senckenbergiana Biologica 65: 321-323. 


\section{APPENDIX}

Comprehensive list of the species considered in this work

We highlight new records $(*)$, species of unlikely occurrence $\left(^{\circ}\right)$ and species of plausible occurrence in need of verification $\left(^{\circ}\right)$.

\section{Agelenidae C. L. Koch, 1837}

Agelena labyrinthica (Clerck, 1757) Allagelena gracilens (C. L. Koch, 1841)

- Coelotes atropos (Walckenaer, 1830)

Coelotes mediocris Kulczyński, 1887

- Coelotes pabulator Simon, 1875

Coelotes pickardi O. P.-Cambridge, 1873

Coelotes rudolf (Schenkel, 1925)

Eratigena agrestis (Walckenaer, 1802)

Histopona leonardoi Bolzern, Pantini \& Isaia, 2013

- Inermocoelotes inermis (L. Koch, 1855)

- Tegenaria domestica (Clerck, 1757)

Tegenaria parietina (Fourcroy, 1785)

Tegenaria silvestris L. Koch, 1872

Textrix denticulata (Olivier, 1789)

AMAUROBIIDAE Thorell, 1870

Amaurobius fenestralis (Ström, 1768)

Amaurobius ferox (Walckenaer, 1830)

Amaurobius jugorum L. Koch, 1868

* Amaurobius similis (Blackwall, 1861)

ANYPHAENIDAE Bertkau, 1878

Anyphaena accentuata (Walckenaer, 1802)

ARANEIDAE Clerck, 1757

Aculepeira carbonaria (L. Koch, 1869)

Aculepeira ceropegia (Walckenaer, 1802)

Agalenatea redii (Scopoli, 1763)

Araneus angulatus Clerck, 1757

Araneus circe (Audouin, 1826)

Araneus diadematus Clerck, 1757

- Araneus marmoreus Clerck, 1757

Araneus quadratus Clerck, 1757

Araneus sturmi (Hahn, 1831)

Araniella cucurbitina (Clerck, 1757)

- Araniella opisthographa (Kulczyński, 1905)

Argiope bruennichi (Scopoli, 1772)

Cyclosa conica (Pallas, 1772)

Gibbaranea bituberculata (Walckenaer, 1802)

* Hypsosinga albovittata (Westring, 1851)

- Hypsosinga heri (Hahn, 1831)

Hypsosinga pygmaea (Sundevall, 1831)
- Larinioides cornutus (Clerck, 1757)

- Larinioides sclopetarius (Clerck, 1757)

Mangora acalypha (Walckenaer, 1802)

- Neoscona adianta (Walckenaer, 1802)

- Nuctenea umbratica (Clerck, 1757)

Parazygiella montana (C. L. Koch, 1834)

Singa hamata (Clerck, 1757)

Zilla diodia (Walckenaer, 1802)

Zygiella $x$-notata (Clerck, 1757)

ATYPIDAE Thorell, 1870

Atypus affinis Eichwald, 1830

* Atypus muralis Bertkau, 1890

\section{Clubionidae Wagner, 1887}

Clubiona alpicola Kulczyński, 1882

Clubiona brevipes Blackwall, 1841

* Clubiona comta C. L. Koch, 1839

* Clubiona corticalis (Walckenaer, 1802)

Clubiona diversa O. P.-Cambridge, 1862

Clubiona frutetorum L. Koch, 1867

- Clubiona hilaris Simon, 1878

Clubiona lutescens Westring, 1851

Clubiona neglecta O. P.-Cambridge, 1862

Clubiona phragmitis C. L. Koch, 1843

Clubiona reclusa O. P.-Cambridge, 1863

Clubiona stagnatilis Kulczyński, 1897

Clubiona terrestris Westring, 1851

CrBAEIDAE Banks, 1892

- Argyroneta aquatica (Clerck, 1757)

Cybaeus intermedius Maurer, 1992

DiCTYNIDAE O. P.-Cambridge, 1871

Dictyna arundinacea (Linnaeus, 1758)

* Dictyna latens (Fabricius, 1775)

DysDeridae C. L. Koch, 1837

Dysdera crocata C. L. Koch, 1838

* Dysdera erythrina (Walckenaer, 1802) 
- Dysdera ninnii Canestrini, 1868

Harpactea hombergi (Scopoli, 1763)

Harpactocrates drassoides (Simon, 1882)

ERESIDAE C. L. Koch, 1845

Eresus kollari Rossi, 1846

EUTICHURIDAE Lehtinen, 1967

Cheiracanthium elegans Thorell, 1875

Cheiracanthium mildei L. Koch, 1864

- Cheiracanthium oncognathum Thorell, 1871

Cheiracanthium punctorium (Villers, 1789)

FilistatidAE Ausserer, 1867

Filistata insidiatrix (Forsskål, 1775)

GNAPHOSIDAE Pocock, 1898

Aphantaulax trifasciata (O. P.-Cambridge, 1872) Berlandina nubivaga (Simon, 1878)

Callilepis nocturna (Linnaeus, 1758)

- Civizelotes gracilis (Canestrini, 1868)

- Drassodes fugax (Simon, 1878)

Drassodes lapidosus (Walckenaer, 1802)

- Drassodes luteomicans (Simon, 1878)

Drassodes pubescens (Thorell, 1856)

Drassodes villosus (Thorell, 1856)

Drassodex drescoi Hervé, Roberts \& Murphy, 2009

- Drassodex heeri (Pavesi, 1873)

- Drassodex hispanus (L. Koch, 1866) Drassyllus praeficus (L. Koch, 1866) Drassyllus pumilus (C. L. Koch, 1839)

Drassyllus pusillus (C. L. Koch, 1833)

* Drassyllus villicus (Thorell, 1875) Echemus angustifrons (Westring, 1861)

- Gnaphosa alpica Simon, 1878 Gnaphosa badia (L. Koch, 1866) Gnaphosa bicolor (Hahn, 1833)

- Gnaphosa leporina (L. Koch, 1866)

* Gnaphosa lucifuga (Walckenaer, 1802) Gnaphosa lugubris (C. L. Koch, 1839) Gnaphosa montana (L. Koch, 1866) Gnaphosa muscorum (L. Koch, 1866)

- Gnaphosa occidentalis Simon, 1878 Gnaphosa petrobia L. Koch, 1872

- Gnaphosa tigrina Simon, 1878 Haplodrassus aenus Thaler, 1984

- Haplodrassus cognatus (Westring, 1861)

* Haplodrassus dalmatensis (L. Koch, 1866)
- Haplodrassus kulczynskii Lohmander, 1942 Haplodrassus signifer (C. L. Koch, 1839) Haplodrassus silvestris (Blackwall, 1833)

* Haplodrassus soerenseni (Strand, 1900) Haplodrassus umbratilis (L. Koch, 1866) Micaria aenea Thorell, 1871 Micaria alpina L. Koch, 1872

* Micaria coarctata (Lucas, 1846) Micaria formicaria (Sundevall, 1831) Micaria fulgens (Walckenaer, 1802) Micaria pulicaria (Sundevall, 1831) Micaria rossica Thorell, 1875

* Micaria silesiaca L. Koch, 1875

* Micaria subopaca Westring, 1861 Nomisia aussereri (L. Koch, 1872)

* Nomisia exornata (C. L. Koch, 1839)

- Parasyrisca vinosa (Simon, 1878)

* Phaeocedus braccatus (L. Koch, 1866)

- Scotophaeus quadripunctatus (Linnaeus, 1758)

- Scotophaeus scutulatus (L. Koch, 1866) Trachyzelotes pedestris (C. L. Koch, 1837)

* Zelotes aeneus (Simon, 1878) Zelotes apricorum (L. Koch, 1876) Zelotes clivicola (L. Koch, 1870) Zelotes devotus Grimm, 1982 Zelotes electus (C. L. Koch, 1839)

* Zelotes gallicus Simon, 1914 Zelotes latreillei (Simon, 1878) Zelotes longipes (L. Koch, 1866)

- Zelotes mundus (Kulczyński, 1897)

* Zelotes paroculus Simon, 1914 Zelotes petrensis (C. L. Koch, 1839) Zelotes subterraneus (C. L. Koch, 1833) Zelotes talpinus (L. Koch, 1872)

HAHNIIDAE Bertkau, 1878

Antistea elegans (Blackwall, 1841) Cryphoeca silvicola (C. L. Koch, 1834) Hahnia nava (Blackwall, 1841)

\section{LINYPHIIDAE Blackwall, 1859}

Agyneta alpica Tanasevitch, 2000

* Agyneta cauta (O. P.-Cambridge, 1902) Agyneta conigera (O. P.-Cambridge, 1863)

- Agyneta gulosa (L. Koch, 1869)

- Agyneta nigripes (Simon, 1884) Agyneta orites (Thorell, 1875) Agyneta rurestris (C. L. Koch, 1836)

- Agyneta saxatilis (Blackwall, 1844) Anguliphantes monticola (Kulczyński, 1881) Araeoncus anguineus (L. Koch, 1869) 
Asthenargus paganus (Simon, 1884)

* Bathyphantes gracilis (Blackwall, 1841)

* Bathyphantes similis Kulczyński, 1894

* Bolyphantes luteolus (Blackwall, 1833) Caracladus avicula (L. Koch, 1869) Centromerita bicolor (Blackwall, 1833) Centromerus brevipalpus (Menge, 1866)

* Centromerus pabulator Simon, 1875 Centromerus subalpinus Lessert, 1907 Centromerus sylvaticus (Blackwall, 1841)

* Ceratinella brevipes (Westring, 1851) Ceratinella brevis (Wider, 1834) Ceratinella scabrosa (O. P.-Cambridge, 1871) Collinsia inerrans (O. P.-Cambridge, 1885) Diplocentria bidentata (Emerton, 1882) Diplocephalus cristatus (Blackwall, 1833)

- Diplocephalus helleri (L. Koch, 1869) Diplocephalus latifrons (O. P.-Cambridge, 1863) Diplostyla concolor (Wider, 1834) Drapetisca socialis (Sundevall, 1833)

* Entelecara acuminata (Wider, 1834)

* Erigone atra Blackwall, 1833 Erigone cristatopalpus Simon, 1884 Erigone dentipalpis (Wider, 1834)

- Erigone remota L. Koch, 1869

* Erigonella subelevata (L. Koch, 1869)

* Floronia bucculenta (Clerck, 1757) Frontinellina frutetorum (C. L. Koch, 1834) Gnathonarium dentatum (Wider, 1834) Gonatium rubens (Blackwall, 1833) Gongylidiellum latebricola (O. P.-Cambridge, 1871)

* Hilaira excisa (O. P.-Cambridge, 1871) Incestophantes frigidus (Simon, 1884)

* Lepthyphantes nodifer Simon, 1884

* Lessertinella kulczynskii (Lessert, 1910)

* Linyphia hortensis Sundevall, 1830 Linyphia triangularis (Clerck, 1757)

* Macrargus rufus (Wider, 1834) Mansuphantes pseudoarciger (Wunderlich, 1985) Maso sundevalli (Westring, 1851)

- Mecynargus brocchus (L. Koch, 1872)

- Mecynargus paetulus (O. P.-Cambridge, 1875)

* Mecopisthes latinus Millidge, 1978

* Mermessus trilobatus (Emerton, 1882) Metopobactrus prominulus (O. P.-Cambridge, 1872)

* Micrargus alpinus Relys \& Weiss, 1997 Micrargus apertus (O. P.-Cambridge, 1871)

* Micrargus herbigradus (Blackwall, 1854)

* Micrargus subaequalis (Westring, 1851)

* Microlinyphia impigra (O. P. Cambridge, 1871) Microlinyphia pusilla (Sundevall, 1830) Microneta viaria (Blackwall, 1841) Minyriolus pusillus (Wider, 1834)

* Mughiphantes armatus (Kulczyński, 1905)

* Mughiphantes cornutus (Schenkel, 1927) Mughiphantes handschini (Schenkel, 1919) Mughiphantes pulcheroides (Wunderlich, 1985)
Neriene clathrata (Sundevall, 1830)

* Neriene emphana (Walckenaer, 1841)

Neriene peltata (Wider, 1834)

- Oedothorax agrestis (Blackwall, 1853)

* Oedothorax apicatus (Blackwall, 1850)

- Oedothorax gibbifer (Kulczyński, 1882)

Oedothorax retusus (Westring, 1851)

- Oreoneta montigena (L. Koch, 1872)

- Oreonetides glacialis (L. Koch, 1872)

- Oreonetides vaginatus (Thorell, 1872) Palliduphantes pallidus (O. P.-Cambridge, 1871) Pelecopsis elongata (Wider, 1834)

* Pelecopsis parallela (Wider, 1834) Pelecopsis radicicola (L. Koch, 1872) Peponocranium orbiculatum (O. P.-Cambridge, 1882) Pityohyphantes phrygianus (C. L. Koch, 1836)

* Pocadicnemis juncea Locket \& Millidge, 1953

* Pocadicnemis pumila (Blackwall, 1841) Porrhomma microphthalmum (O. P.-Cambridge, 1871)

* Porrhomma pygmaeum (Blackwall, 1834)

* Prinerigone vagans (Audouin, 1826)

* Scotinotylus alpigena (L. Koch, 1869)

- Scotinotylus antennatus (O. P.-Cambridge, 1875) Stemonyphantes lineatus (Linnaeus, 1758)

* Tapinocyba insecta (L. Koch, 1869) Tapinocyba maureri Thaler, 1991 Tapinocyba pallens (O. P.-Cambridge, 1872) Tenuiphantes flavipes (Blackwall, 1854)

* Tenuiphantes mengei (Kulczyński, 1887) Tenuiphantes tenebricola (Wider, 1834) Tenuiphantes tenuis (Blackwall, 1852) Tiso vagans (Blackwall, 1834)

* Trichoncus sordidus Simon, 1884 Trichopterna cito (O. P.-Cambridge, 1872) Troglohyphantes lucifuga (Simon, 1884) Troglohyphantes nigraerosae Brignoli, 1971 Troglohyphantes sarae Pesarini, 2011 Troxochrus scabriculus (Westring, 1851)

- Typhocrestus digitatus (O. P.-Cambridge, 1872) Walckenaeria acuminata Blackwall, 1833 Walckenaeria alticeps (Denis, 1952) Walckenaeria antica (Wider, 1834) Walckenaeria atrotibialis (O. P.-Cambridge, 1878) Walckenaeria furcillata (Menge, 1869)

- Walckenaeria monoceros (Wider, 1834) Walckenaeria nodosa O. P.-Cambridge, 1873 Walckenaeria obtusa Blackwall, 1836

- Walckenaeria vigilax (Blackwall, 1853)

\section{LIOCRANIDAE Simon, 1897}

Agroeca cuprea Menge, 1873 Agroeca proxima (O. P.-Cambridge, 1871) Liocranum rupicola (Walckenaer, 1830)

* Mesiotelus tenuissimus (L. Koch, 1866) Scotina celans (Blackwall, 1841) 
Alopecosa accentuata (Latreille, 1817)

Alopecosa aculeata (Clerck, 1757)

Alopecosa cuneata (Clerck, 1757)

* Alopecosa cursor (Hahn, 1831)

Alopecosa fabrilis (Clerck, 1757)

Alopecosa pulverulenta (Clerck, 1757)

Alopecosa sulzeri (Pavesi, 1873)

* Alopecosa taeniata (C. L. Koch, 1835) Alopecosa trabalis (Clerck, 1757)

Arctosa figurata (Simon, 1876)

Arctosa leopardus (Sundevall, 1833)

* Arctosa lutetiana (Simon, 1876)

- Arctosa perita (Latreille, 1799)

* Arctosa personata (L. Koch, 1872) Arctosa renidescens Buchar \& Thaler, 1995

Aulonia albimana (Walckenaer, 1805)

Hogna radiata (Latreille, 1817)

* Pardosa agrestis (Westring, 1861)

- Pardosa agricola (Thorell, 1856)

Pardosa alacris (C. L. Koch, 1833)

Pardosa amentata (Clerck, 1757)

Pardosa bifasciata (C. L. Koch, 1834)

Pardosa blanda (C. L. Koch, 1833)

Pardosa ferruginea (L. Koch, 1870)

Pardosa giebeli (Pavesi, 1873)

Pardosa hortensis (Thorell, 1872)

Pardosa lugubris (Walckenaer, 1802)

Pardosa mixta (Kulczyński, 1887)

- Pardosa monticola (Clerck, 1757)

- Pardosa morosa (L. Koch, 1870)

Pardosa nigra (C. L. Koch, 1834)

- Pardosa oreophila Simon, 1937

- Pardosa paludicola (Clerck, 1757)

Pardosa palustris (Linnaeus, 1758)

Pardosa prativaga (L. Koch, 1870)

Pardosa proxima (C. L. Koch, 1847)

- Pardosa pullata (Clerck, 1757)

Pardosa riparia (C. L. Koch, 1833)

Pardosa saturatior Simon, 1937

Pardosa schenkeli Lessert, 1904

Pardosa torrentum Simon, 1876

* Pardosa vlijmi Den Hollander \& Dijkstra, 1974

Pardosa wagleri (Hahn, 1822)

* Pirata piraticus (Clerck, 1757)

* Pirata tenuitarsis Simon, 1876

* Piratula hygrophila (Thorell, 1872)

Piratula latitans (Blackwall, 1841)

* Trochosa hispanica Simon, 1870

Trochosa ruricola (De Geer, 1778)

Trochosa terricola Thorell, 1856

Xerolycosa miniata (C. L. Koch, 1834)

Xerolycosa nemoralis (Westring, 1861)
* Ero aphana (Walckenaer, 1802)

* Ero furcata (Villers, 1789)

MiturgidAe Simon, 1886

- Zora manicata Simon, 1878

Zora silvestris Kulczyński, 1897

Zora spinimana (Sundevall, 1833)

Nesticidae Simon, 1894

Nesticus cellulanus (Clerck, 1757)

Nesticus eremita Simon, 1879

OECOBIIDAE Blackwall, 1862

Oecobius cellariorum (Dugès, 1836)

OXYOPIDAE Thorell, 1870

Oxyopes ramosus (Martini \& Goeze, 1778)

PhILODROMIDAE Thorell, 1870

Philodromus aureolus (Clerck, 1757)

Philodromus cespitum (Walckenaer, 1802)

Philodromus collinus C. L. Koch, 1835

* Philodromus emarginatus (Schrank, 1803)

- Philodromus fuscolimbatus Lucas, 1846

- Philodromus laricium Simon, 1875

Philodromus margaritatus (Clerck, 1757)

- Philodromus poecilus (Thorell, 1872)

Philodromus praedatus O. P.-Cambridge, 1871

Philodromus vagulus Simon, 1875

- Thanatus arenarius L. Koch, 1872

Thanatus atratus Simon, 1875

Thanatus coloradensis Keyserling, 1880

Thanatus formicinus (Clerck, 1757)

- Thanatus oblongiusculus (Lucas, 1846)

- Thanatus striatus C. L. Koch, 1845

Tibellus oblongus (Walckenaer, 1802)

Pholcidae C. L. Koch, 1850

Pholcus phalangioides (Fuesslin, 1775) 
Phrurolithidae Banks, 1892

Phrurolithus festivus (C. L. Koch, 1835)

Phrurolithus minimus C. L. Koch, 1839)

Pisauridae Simon, 1890

- Dolomedes fimbriatus (Clerck, 1757)

Pisaura mirabilis (Clerck, 1757)

SALTiCIDAE Blackwall, 1841

Aelurillus v-insignitus (Clerck, 1757)

Ballus chalybeius (Walckenaer, 1802)

* Chalcoscirtus infimus (Simon, 1868)

Euophrys frontalis (Walckenaer, 1802)

Evarcha arcuata (Clerck, 1757)

Evarcha falcata (Clerck, 1757)

Evarcha laetabunda (C. L. Koch, 1846)

Heliophanus aeneus (Hahn, 1832)

- Heliophanus auratus C. L. Koch, 1835

Heliophanus cupreus (Walckenaer, 1802)

Heliophanus flavipes (Hahn, 1832)

Heliophanus kochii Simon, 1868

Heliophanus lineiventris Simon, 1868

Heliophanus tribulosus Simon, 1868

* Marpissa nivoyi (Lucas, 1846)

* Mendoza canestrinii (Ninni, 1868)

- Myrmarachne formicaria (De Geer, 1778)

Neon reticulatus (Blackwall, 1853)

- Pellenes lapponicus (Sundevall, 1833)

Pellenes tripunctatus (Walckenaer, 1802)

Philaeus chrysops (Poda, 1761)

Phlegra fasciata (Hahn, 1826)

Pseudeuophrys erratica (Walckenaer, 1826)

Pseudeuophrys lanigera (Simon, 1871)

Pseudeuophrys vafra (Blackwall, 1867)

* Pseudicius encarpatus (Walckenaer, 1802)

Saitis barbipes (Simon, 1868)

- Salticus cingulatus (Panzer, 1797)

Salticus scenicus (Clerck, 1757)

Salticus zebraneus (C. L. Koch, 1837)

Sitticus longipes (Canestrini, 1873)

Sitticus rupicola (C. L. Koch, 1837)

Sitticus saxicola (C. L. Koch, 1846)

Talavera aequipes (O. P.-Cambridge, 1871)

SCYTODIDAE Blackwall, 1864

Scytodes thoracica (Latreille, 1802)
Segestriddae Simon, 1893

Segestria bavarica C. L. Koch, 1843

Segestria senoculata (Linnaeus, 1758)

SPARASSIDAE Bertkau, 1872

Micrommata virescens (Clerck, 1757)

Tetragnathidae Menge, 1866

Meta menardi (Latreille, 1804)

Metellina mengei (Blackwall, 1870)

Metellina merianae (Scopoli, 1763)

Metellina segmentata (Clerck, 1757)

Pachygnatha clercki Sundevall, 1823

Pachygnatha degeeri Sundevall, 1830

Pachygnatha listeri Sundevall, 1830

Tetragnatha extensa (Linnaeus, 1758)

Tetragnatha montana Simon, 1874

- Tetragnatha obtusa C. L. Koch, 1837

- Tetragnatha pinicola L. Koch, 1870

THERIDIIDAE Sundevall, 1833

Asagena phalerata (Panzer, 1801)

Crustulina guttata (Wider, 1834)

* Dipoena melanogaster (C. L. Koch, 1837)

Dipoena torva (Thorell, 1875)

Enoplognatha ovata (Clerck, 1757) vel Enopognatha latimana Hippa \& Oksala, 1982

Enoplognatha thoracica (Hahn, 1833)

Episinus maculipes Cavanna, 1876

Episinus truncatus Latreille, 1809

Euryopis flavomaculata (C. L. Koch, 1836)

Parasteatoda lunata (Clerck, 1757)

Parasteatoda tepidariorum (C. L. Koch, 1841)

Pholcomma gibbum (Westring, 1851)

Phylloneta impressa (L. Koch, 1881)

Phylloneta sisyphia (Clerck, 1757)

Robertus arundineti (O. P.-Cambridge, 1871)

Robertus lividus (Blackwall, 1836)

Robertus neglectus (O. P.-Cambridge, 1871)

* Robertus scoticus Jackson, 1914

Robertus truncorum (L. Koch, 1872)

Rugathodes bellicosus (Simon, 1873)

Steatoda albomaculata (De Geer, 1778)

Steatoda bipunctata (Linnaeus, 1758)

- Steatoda grossa (C. L. Koch, 1838)

Steatoda triangulosa (Walckenaer, 1802)

Theridion melanurum Hahn, 1831

Theridion petraeum L. Koch, 1872

Theridion pictum (Walckenaer, 1802)

Theridion varians Hahn, 1833 
THOMISIDAe Sundevall, 1833

Cozyptila blackwalli (Simon, 1875)

Diaea dorsata (Fabricius, 1777)

Ebrechtella tricuspidata (Fabricius, 1775)

Heriaeus hirtus (Latreille, 1819)

Misumena vatia (Clerck, 1757)

Ozyptila atomaria (Panzer, 1801)

Ozyptila praticola (C. L. Koch, 1837)

Ozyptila pullata (Thorell, 1875)

Ozyptila rauda Simon, 1875

* Ozyptila secreta Thaler, 1987

* Ozyptila simplex (O. P.-Cambridge, 1862)

Ozyptila trux (Blackwall, 1846)

- Runcinia grammica (C. L. Koch, 1837)

* Synema globosum (Fabricius, 1775)

Thomisus onustus Walckenaer, 1805

Tmarus piger (Walckenaer, 1802)

Xysticus audax (Schrank, 1803)

Xysticus bifasciatus C. L. Koch, 1837

- Xysticus bliteus (Simon, 1875)

- Xysticus cor Canestrini, 1873

Xysticus cristatus (Clerck, 1757)

Xysticus desidiosus Simon, 1875

Xysticus erraticus (Blackwall, 1834)

Xysticus gallicus Simon, 1875

Xysticus kochi Thorell, 1872
Xysticus lanio C. L. Koch, 1835

Xysticus ninnii Thorell, 1872

- Xysticus nubilus Simon, 1875

Xysticus robustus (Hahn, 1832)

Titanoecidae Lehtinen, 1967

- Titanoeca nivalis Simon, 1874

Trachelidae Simon, 1897

Cetonana laticeps (Canestrini, 1868)

UlOBORIDAE Thorell, 1869

Uloborus plumipes Lucas, 1846

- Uloborus walckenaerius Latreille, 1806

ZODARIIDAE Thorell, 1881

Zodarion italicum (Canestrini, 1868)

Zodarion rubidum Simon, 1914 
